\title{
Versuche über Schub bei Querbiegung
}

Working Paper

Author(s):

Kaufmann, Josef; Menn, Christian

Publication date:

1976

Permanent link:

https://doi.org/10.3929/ethz-a-000099541

Rights / license:

In Copyright - Non-Commercial Use Permitted

Originally published in:

Bericht / Institut für Baustatik und Konstruktion ETH Zürich 7201(1) 
Versuche über Schub bei Querbiegung

Josef Kaufmann

Christian Menn 
(C) Birkhäuser Verlag Basel und Stuttgart ISBN 3-7643-0907-5 


\title{
Versuche über Schub bei Querbiegung
}

\author{
von \\ Josef Kaufmann, dipl. Ing. \\ Prof. Dr. Christian Menn \\ Institut für Baustatik und Konstruktion \\ Eidgenössische Technische Hochschule Zürich
}


1. Einleitung

1.1 Problemstellung und Zielsetzung

1.2 Versuchsprogramm

2. Versuchsträger 3

2.1 Beschreibung 3

2.1.1 Abmessungen der Versuchsträger und Lastanordnung 3

2.1 .2 Bewehrung 3

2.1 .3 Herstellung 3

2.2 Baustoffe. 4

2.2.1 Bewehrungsstahl 4

2.2 .2 Beton 4

2.3 Rechnerische Werte 4

2.3.1 Schubbruchlast $\quad 4$

2.3.2 Querbiegebruchlast 5

2.3.3 Gebrauchslasten 5

3. Versuchsdurchführung 6

3.1 Versuchsanlage 6

3.1 .1 Belastung für Längsbeanspruchung 6

3.1.2 Belastung für Querbeanspruchung 6

3.1.3 Lagerung 6

3.1.4 Messeinrichtungen 6

3.2 Versuchsablauf 7

4. Ergebnisse 8

4.1 Tragverhalten im Gebrauchs- und Bruchzustand 8

4.2 Beanspruchung der Bewehrung 9

4.2.1 Belastungs-Dehnungs-Diagramme

4.2.2 Beanspruchung der Bewehrung im Gebrauchszustand 10

4.2.3 Bruch infolge Querbiegung 11

4.3 Beanspruchung des Betons 11

4.3.1 Druckflansch 11

4.3.2 Betondruckdiagonalen im Steg 11

4.4 Verformungen 12

4.4 .1 Schiebungen 12

4.4.2 Durchbiegungen 12 
4.5 Rissverhalten 12

4.5.1 Risseverlauf $\quad 12$

4.5.2 Rissweiten 13

4.6 Vergleich zwischen Norm und Versuchen 13

4.6.1 Vergleich der experimentellen Bruchlast mit der duktilen Schubtragfähigkeit bei reiner Längsbeanspruchung

4.6.2 Vergleich der experimentellen Bruchlast mit der Schubtragfähigkeit überarmierter Querschnitte (obere Schubspannungsgrenze bei reiner Längsbeanspruchung)

5. Schlussfolgerungen 15

5.1 Dimensionierung der Bewehrung 15

5.2 Betonabmessungen $\quad 15$

ZUSAMMENFASSUNG

$\begin{array}{ll}\text { RESUME } & 17\end{array}$

$\begin{array}{lc}\text { SUMMARY } & 18\end{array}$

$\begin{array}{ll}\text { VERDANKUNGEN } & 19\end{array}$

$\begin{array}{lr}\text { LITERATURVERZEICHNIS } & 20\end{array}$

$\begin{array}{ll}\text { BEZEICHNUNGEN } & 21\end{array}$

$\begin{array}{ll}\text { TABELLEN } 1 \text { BIS } 8 & 24\end{array}$

BILDER 1 BIS 91 


\subsection{Problemstellung und Zielsetzung}

Eine wirklichkeitsnahe Ermittlung der Querschnittstragfähigkeit lässt sioh nur unter Berücksichtigung aller gleichzeitig wirkenden Schnittkräfte durchführen. Für beliebige Kombinationen von Biegung, Normalkraft, Querkraft und Torsion liegen heute zuverlässige Forschungsergebnisse vor; insbesondere befassen sich neuere Arbeiten sehr eingehend mit der Bestimmung der Schubtragfähigkeit bei gleichzeitiger Wirkung sämtlicher Stabschnittkräfte. Der Einfluss einer zusätzlich überlagerten Querbiegung ist dagegen bisher weder theoretisch noch experimentell zufriedenstellend abgeklärt worden.

Vor allem im Brückenbau spielt die Beanspruchungskombination "Schub in Längsrichtung mit überlagerter Querbiegung" eine wichtige Rolle, da die schubbeanspruchten Hauptträgerstege durch das Zusammenwirken mit der Fahrbahnplatte praktisch immer eine mehr oder weniger grosse Querbiegung aufnehmen müssen. Hier stellen sich nun zwei für die Querschnittsbemessung wichtige Fragen:

1. Muss die vertikale Stegbewehrung für Schub und Querbiegung getrennt bemessen werden?

2. Muss die zur Bestimmung der Stegstärke massgebende Schubspannungsgrenze bei dieser Beanspruchungskombination abgemindert werden?

Das Ziel der vorliegenden Forschungsarbeit bestand darin, diese beiden wichtigen Fragen in umfangreichen experimentellen Untersuchungen zu prüfen und Grundlagen für eine theoretische Modellbildung zu erarbeiten.

\subsection{Versuchsprogramm}

Das experimentelle Forschungsprogramm über das Schubtragverhalten bei Querbiegung ist in Tabelle 1 zusammengestelit. Es umfasst die Prüfung von sechs I-färmigen Stahlbetonbalken mit je zwei Bruchversuchen pro Träger. Die schubbeanspruchten Bereiche der Versuchsbalken wiesen eine stark unterschiedliche Stegbewehrung auf, so dass in der Laststellung I das duktile Bruchverhalten, und in der Laststellung II das Sprädbruchverhalten geprüft werden konnte. Beim duktilen Verhalten in Laststellung I liess sich somit die Frage der Ueberlagerung der Bewehrungsquerschnitte infolge Schub und Querbiegung abklären, während beim Sprödbruchverhalten in Laststellung II der Einfluss der Querbiegung auf die Schubspannungsgrenze geprüft werden konnte.

Bei den Trägern $S_{1}$ bis $S_{5}$ wurde in den beiden Laststellungen I und II der Bruch bei konstanter Querbiegung ( $\left.m_{z u l}\right)$ durch Steigerung der Querkraft erreicht. Die Prüfung des Trägers $S_{6}$ erfolgte in Laststellung I in gleicher Weise. In Laststellung II hingegen führte die Steigerung der Querbiegung bei konstanter Querkraft (Q zul) zum Bruch des Trägers. 
Parameter der Versuchsserie waren einerseits die Schubkennwerte

- Bügelbewehrung

- Längsbewehrung

- Diagonalenneigung

und andererseits die Querbiegekennwerte

- Verhältnis der Bügelbewehrung auf der Zug- und der Druckseite

- Grösse der Querbiegebeanspruchung.

Ausgehend vom Vergleichsträger 5 , variierte bei den übrigen Versuchen jeweils nur ein Parameter, so dass sich das durch die Querbiegung veränderte Schubtragverhalten sofort feststellen liess. 


\subsubsection{Abmessungen der Versuchsträger und Lastanordnung}

Die. Trägerabmessungen und die Anordnung der Belastungen für die Längs- und Querbeanspruchung sind aus den Bildern 1, 2 und 3 ersichtlich. Die Träger wiesen im Lastfall I eine Spannweite von $6.0 \mathrm{~m}$ und im Lastfall II eine verkürzte Spannweite von $4.0 \mathrm{~m}$ auf. Der I-förmige Trägerquerschnitt war $62 \mathrm{~cm}$ hoch, die Flansche $70 \mathrm{~cm}$ breit. Die Stegstärke betrug $10 \mathrm{~cm}$. Damit liess sich bei einer möglichst hohen Beanspruchung des Betonquerschnittes die zweischnittige Bügelbewehrung gerade noch einwandfrei anordnen.

Die Flansche waren beim Steganschluss $9 \mathrm{~cm}$ stark und verjüngten sich bis zum Flanschrand auf $7 \mathrm{~cm}$.

Die Belastung für die Beanspruchung in Längsrichtung erfolgte mit zwei $100 \mathrm{t}$ Pressen, die in Laststellung $I$ in einem Abstand von $2 \mathrm{~m}$ symmetrisch zur Trägermitte angeordnet waren. In Laststellung II wurde das Auflager im geprüften Bereich umgesetzt, die Spannweite dadurch auf $4.0 \mathrm{~m}$ verkürzt und nur noch eine Hauptlast-Presse eingesetzt.

Die Belastung für die Querbiegung wurde durch sechs Sekundärpressen über Hebelarme und Verteilbalken punktförmig in Abständen von $55 \mathrm{~cm}$ längs des ganzen Trägers auf die Flanschränder übertragen.

\subsubsection{Bewehrung}

Die Bewehrung der Versuchsträger ist in den Bildern 8 bis 10 dargestellt; sie bestand bei allen Balken aus naturhartem, profiliertem Stahl (Box-Ultra). Die Betonüberdeckung der Querbewehrung betrug $1 \mathrm{~cm}$, diejenige der Längsbewehrung $2 \mathrm{~cm}$.

Die Bügelbewehrung liess sich bei der geringen Stegstärke und der unterschiedlichen Querschnittsfläche auf der Zug- und Druckseite nicht geschlossen ausbilden. Im Hinblick auf eine einwandfreie Einleitung der Querbiegung wurden die Bügel bis zum Rand der Flansche verlängert.

Die Abstufung der Längsbewehrung entsprach dem Verlauf der Zugkraft im Untergurt. Zur Elimination von Unsicherheiten in der Kraftübertragung bei üblichen Armierungsstössen wurden die einzelnen Bewehrungsstäbe, welche unterschiedliche Durchmesser aufwiesen, stumpf zusammengeschweisst. Damit sich der Bruch gut lokalisieren liess, wurden die Auflagerbereiche und die Zonen ausserhalb der Prüfstellen überdimensioniert.

\subsubsection{Herstellung}

Die Schalung der Versuchsträger bestand aus einer massiven Holzkonstruktion. Distanzhalter und eine kräftige Vorspannung gewährleisteten eine einwandfreie Lage der Bewehrung und eine massgerechte Herstellung der Träger.

Die Träger wurden liegend betoniert. Zur Verdichtung des Betons im dünnen, stark bewehrten Querschnitt dienten sechs Schalungsvibratoren. Dank sorgfältigem Einbringen und Verdichten liess sich auch im unteren, vollständig eingeschalten Flansch ein einwandfreier Beton mit ausreichender Festigkeit herstellen. Bis zur Versuchsdurchfüh- 
rung wurden die Träger bei einer konstanten Temperatur von $20^{\circ} \mathrm{C}$ während 28 Tagen gelagert.

2.2 Baustoffe

2.2.1 Bewehrungsstahl

In Tabelle 2 sind die Festigkeitswerte des verwendeten Bewehrungsstahles zusammengefasst. Die Streuungen bei Stählen mit gleichem Durchmesser waren klein, die Mittelwerte somit repräsentativ. In $\theta i l d 11$ ist ein typisches Spannungs-Dehnungs-Diagramm aufgezeichnet. Da die Kurzzeit-Festigkeitswerte bei verschiedenen Dehnungsgeschwindigkeiten $\dot{\varepsilon}$ stark variieren, wurden der Auswertung die sogenannten statischen Werte $(\dot{\varepsilon}=0)$ zugrunde gelegt. Diese Werte ergaben sich aus den in [2] beschriebenen Zugversuchen.

\section{$\underline{2.2 .2 \text { Beton }}$}

Die Zusammensetzung des mit schweizerischem Portlandzement hergestellten Betons war bei allen Versuchsträgern dieselbe (Tabelle 3). Die Sieblinie entsprach dabei der EMPA-Kurve.

Zur Ermittlung der Betonfestigkeit wurden mit jedem Balken gleichzeitig sechs Prismen $(12 \times 12 \times 36 \mathrm{~cm})$ hergestellt und zum Zeitpunkt des Versuches jeweils an einem Prisma der Elastizitätsmodul und die Prismendruckfestigkeit, an den übrigen Probekörpern die Biegezug- und Würfeldruckfestigkeit ermittelt. Diese bei relativ hoher Dehnungsgeschwindigkeit gemessenen Werte sind aus Tabelle $4 \mathrm{zu}$ entnehmen.

\subsection{Rechnerische Werte}

Die folgenden Angaben über die rechnerischen Werte zur Bestimmung der theoretischen Bruchlasten und der Gebrauchslasten beziehen sich auf den eigentlichen Prüfbereich. Auflager- und Zwischenzonen waren stärker bemessen. Die theoretischen Werte wurden für reine Längsbeanspruchung, bzw. reine Querbeanspruchung, d.h. ohne Interaktion, ermittelt.

Die Bewehrungsquerschnitte sind in den Bildern 8 bis 10 angegeben. Der in Tabelle 1 aufgeführte rechnerische Bewehrungsgehalt der Bügel wurde wie folgt festgelegt:

$$
\mu_{B}(\%)=\frac{\mathrm{Fe}_{B}}{\mathrm{~b}_{0} \cdot \mathrm{s}_{B}} \cdot 100 \%
$$

\section{$\underline{2.3 .1 \text { Schubbruchlast }}$}

Die Berechnung der theoretischen Traglast bei reiner Längsbeanspruchung erfolgte nach Norm SIA 162, Richtlinie 34. In Anbetracht der relativ hohen nominellen Schubbeanspruchung der Balken, wurde der Schubwiderstandsanteil $Q_{b}$ nicht berücksichtigt. Mit der Diagonalenneigung a als Parameter ergab sich somit

$$
Q_{U}^{T h}=B \cdot \frac{h}{s_{B}} \cdot \frac{1}{\operatorname{tg} \alpha}
$$


oder

$$
Q_{u}^{T h}=\sigma_{f s} \cdot \mu_{B} \cdot \frac{b_{0} \cdot h}{\operatorname{tg} \alpha}
$$

wobei $\sigma_{f s}$ der effektiven, im Zugversuch ermittelten Fliessspannung der Bügelbewehrung entspricht. Die Werte der theoretischen Schubbruchlasten sind in Tabelle.8 zusammengestellt.

Mit der Parametervariation liess sich die Schubbewehrung, die Diagonalenneigung und damit auch die theoretische Zugkraftlinie $Z_{U}$ im Untergurt bestimmen.

$$
Z_{U}=\frac{M}{h}+\frac{Q}{2 \cdot \operatorname{tg} \alpha}
$$

Damit sich der Schubbruchmechanismus auf der ganzen Länge des Prüfbereiches ausbilden kannte, wurde die Längsbewehrung im Untergurt in Abständen von $20 \mathrm{~cm}$ der Zugkraftlinie entsprechend abgestuft und, wie schon erwähnt, Stäbe verschiedenen Durchmessers stumpf aneinander geschweisst.

\subsubsection{Querbiegebruchlast}

Die Bestimmung der theoretischen Querbiegebruchlast bei reiner Querbeanspruchung erfolgte unter Berücksichtigung der entsprechenden Zug- und Druckbewehrung. Diese Werte sind in Tabelle 8 enthalten.

\subsubsection{Gebrauchslasten}

Die Gebrauchsbelastung bei reiner Längs- oder Querbeanspruchung wurde nicht aufgrund zulässiger Spannungen ermittelt, sondern durch Abminderung der Bruchlast um den effektiven Sicherheitskoeffizienten, der sich aus dem üblichen Sicherheitsbeiwert 1.8 sowie der Berücksichtigung der effektiven und der der Norm SIA 162 zugrunde gelegten Stahlfliessspannung ergibt.

$$
s_{\text {eff }}=1.8 \cdot \frac{\sigma_{f s}}{\sigma_{f, \text { norm }}}
$$

Die in Tabelle 8 angegebenen zulässigen Beanspruchungen liessen sich damit folgendermassen bestimmen:

$$
\begin{aligned}
& Q_{z u l}=\frac{Q_{u}^{T h}}{s_{e f f}} \\
& m_{z u l}=\frac{m_{u}^{T h}}{s_{e f f}}
\end{aligned}
$$




\section{VERSUCHSDURCHFUEHRUNG}

\subsection{Versuchsanlage}

Die Balkenversuche wurden auf dem Aufspannboden der Prüfhalle der Eidgenössischen Materialprüfungs - und Versuchsanstalt (EMPA) in Dübendorf durchgeführt.

Die Versuchsanlage bestand im wesentlichen aus den Belastungseinrichtungen für die Längs- und Querbeanspruchung, den Lagern und den Mess- und Registrierinstrumenten.

\subsubsection{Belastung für Längsbeanspruchung}

Die Belastung für die Beanspruchung in Längsrichtung wurde mit zwei im Abstand von 2 m symmetrisch zur Balkenmitte angeordneten 100 t Pressen aufgebracht. Die beiden Pressen waren an Querrahmen befestigt und gaben ihre Last über Kugelgelenke genau in der Balkenschwerachse ab.

\subsubsection{Belastung für Querbeanspruchung}

Das Prinzip der Belastungseinrichtung für Querbiegung ist in Bild 3 dargestellt. Die Zugpressen befanden sich zwischen zwei als Hebelarme wirkenden I-Stahlträgern, welche die Belastung über Verteilbalken punktförmig in Abständen von $55 \mathrm{~cm}$ längs des Trägers auf die Flanschränder übertrugen. Bei einer Lastausbreitung von $45^{\circ}$ ergab sich ein konstantes Querbiegemoment im Trägersteg. Die Zugpressen wurden derart gesteuert, dass des Querbiegemoment auf der ganzen Balkenlänge konstant war.

Dieses Belastungssystem erzeugte zusätzlich zur Querbiegung eine Normalkraft im Steg, die allerdings infolge der relativ gross gewählten Hebelarme sehr klein war. Bei einem Querbiegemoment von $\mathrm{m}_{\mathrm{q}}=1.0 \mathrm{mt} / \mathrm{m}$ und einer Bügelbewehrung vom Durchmesser $8 \mathrm{~mm}$ $\left(s_{B}=20 \mathrm{~cm}\right)$, entstand im Stahl infolge dieser Normalkraft eine Spannung von

$$
\Delta \sigma_{Q}=\frac{m_{G}}{a} \cdot \frac{{ }^{5} B}{2 \cdot F e_{B}}=0.17 \mathrm{t} / \mathrm{cm}^{2} .
$$

Verglichen mit der Fliessspannung entsprach dies einem Fehler van 3\%.

\section{1 .3 Lagerung}

Die Balken waren auf allseitig kippbaren Gleitlagern, bestehend aus einem Kreuzgelenk und einem Teflonlager, abgestützt. Eine Stahlrohr-Haltestange fixierte den Balken in Längsrichtung und diente zur Aufnahme des Horizontalschubes bei einer eventuellen Schiefstellung der Hauptpressen.

Die Stabilisierung des Balkens in Querrichtung erfolgte bei den Auflagern durch seitliche Anschläge am unteren Flansch und beim Ansatz der Hauptpressen durch je eine Zugstange am oberen Flansch.

Das Drehmoment infolge der exzentrischen Querbelastungseinrichtung wurde durch Flanschbiegung auf die oben erwähnten seitlichen Anschläge resp. die Zugstangen abgetragen. 


\subsubsection{Messeinrichtungen}

Aus Bild 15 ist die Anordnung der Messstellen ersichtlich. Die Messung der Kraft $H_{0}$, des Querbiegemomentes und der Betonstauchungen erfolgte mittels Dehnungsmessstreifen, diejenige der Bügeldehnungen und der Dehnungen der Längsbewehrung mittels Deformetern von $20 \mathrm{~cm}$. Für die Messung der Schiebungen wurden Deformeter von $28 \mathrm{~cm}$ verwendet.

Eine elektronische Datenerfassungsanlage speicherte die Messwerte auf einen Lochstreifen, der später mit dem Computer des RZETH ausgewertet werden konnte. Dieses Vorgehen ermöglichte ein rasches Aufzeichnen sämtlicher Dehnungen und der daraus resultierenden Verformungen.

\subsection{Versuchsablauf}

Eine erste Prüfung der Balken erfolgte in Laststellung I (Spannweite $6 \mathrm{~m}$, Längsbelastung mit beiden Hauptpressen). Nach dem duktilen Bruch des entsprechend bemessenen Schubbereiches wurde ein Balkenlager umgesetzt und dadurch in der Laststellung II (Spannweite $4 \mathrm{~m}$, Längsbelastung mit einer Hauptpresse) das Sprödbruchverhalten des anderen Schubbereiches untersucht.

In Bild 13 ist der generelle Belastungsablauf dargestellt. Bis zum Erreichen der Gebrauchslast erfolgte die Laststeigerung in kleinen Stufen, wobei dem Rissbild und den Rissweiten besondere Aufmerksamkeit geschenkt wurde. Nach dem Entstehen grosser Verformungen musste von der lastgesteuerten zur deformationsgesteuerten Belastung übergegangen werden.

Eine gleichzeitige Steigerung der Längs- und Querbelastung war aus technischen Gründen nicht mäglich. Deshalb wurde vorerst die Längsbelastung und anschliessend in mehreren Stufen die Querbelastung aufgebracht. Nach einigen Lastwechseln im Gebrauchsbereich erfolgte eine Steigerung der Querbiegung bis auf den durch die Parametervariation festgelegten Wert. Anschliessend wurde der Balken durch Erhöhung der Längsbelastung zum Bruch gebracht. 


\section{ERGEBNISSE}

4.1 Tragverhalten im Gebrauchs - und Bruchzustand

Die Tabellen 5 bis 7 enthalten die Anfangs- und Endwerte der Querkraft bei jeder Laststufe sowie die zugehörige Querbiegebelastung. Die angegebenen Werte entsprechen der Querkraft (inkl. Eigengewicht) in einem $1.5 \mathrm{~m}$ vom Auflager entfernten Schnitt. Da die bei Querbiegung wirkende Normalkraft im Trägersteg sehr klein war, wurde sie nicht berücksichtigt.

Der generelle zeitliche Ablauf einer Laststufe ist aus Bild 14 ersichtlich. Die Querkraft wurde vorerst auf den vorgesehenen Wert $Q_{A}$ gesteigert und während zwei Minuten konstant gehalten. Anschliessend erfolgte die Laststeuerung so, dass sich die Deformationen im Verlauf der Messungen nicht veränderten. Dadurch sank die Querkraft auf den Wert $Q_{E}$ ab; $m_{q}$ blieb hingegen praktisch konstant.

Einen generellen Ueberblick über das Tragverhalten vermitteln die Bilder 43 bis 47 und 58 (Verlauf der Durchbiegung in Balkenmitte) und die Bilder 48 bis 57 und 59 (Durchbiegung längs des Balkens bei verschiedenen Laststufen).

\section{Laststellung I}

Der allgemeine Belastungsablauf ist in Bild 13 dargestellt.

Erste Belastungsphase:

In der ersten Phase wurde der Träger bis zum Gebrauchszustand belastet. Zunächst verformte sich der Balken im ungerissenen Zustand nur sehr wenig. Nach Auftreten der ersten Schubrisse fiel die Steifigkeit ab und nahm nach der Ausbildung des Schubfachwerkes einen konstanten wert an.

Zweite Belastungsphase:

Die zweite Phase war durch wiederholte Belastungen im Bereich des Gebrauchszustandes gekennzeichnet. Dabei blieb die Steifigkeit in Längsrichtung bei einer Querkraftsteigerung mit ader ohne gleichzeitig wirkender Querbiegung unverändert.

Dritte Belastungsphase:

Die Belastung während der dritten Phase führte zum Bruch der Balken infolge Querkraft, wobei vorerst die Querbiegung auf den durch die Parametervariation festgelegten Wert gesteigert wurde. Bei der anschliessenden Erhöhung der Querkraft über den Gebrauchszustand blieb die im gerissenen Zustand festgestellte Längssteifigkeit zunächst erhalten, nahm dann jedoch bei der weiteren Laststeigerung kontinuierlich ab, bis sie beim Fliessen der Längsbewehrung den Nullwert erreichte.

In den. Lastverformungskurven lassen sich zwei ausgeprägte Knickpunkte feststellen. Ein erster starker Steifigkeitsabfall, verursacht durch das Fliessen der Bügel auf der Zugseite, ist bei 70 bis $90 \%$ der Bruchlast erkennbar. Nach diesem Fliessbeginn, der wesentlich über dem errechneten Wert aus Superposition von Querkraft und Querbiegung liegt, muss die zusätzliche Last durch andere Tragelemente übernommen werden, was ein weicheres Tragverhalten zur Folge hat. Der zweite markante knickpunkt in der 
Verformungskurve wird durch den Fliessbeginn der Bügel auf der Druckseite verursacht. Mit der Ausbildung eines Biegeschubmechanismus auf einer Länge von 1.0 bis $1.5 \mathrm{~m}$ verformten sich die Balken auch ohne wesentliche Steigerung der Querkraft sehr stark.

Der Bruch der Balken erfolgte infolge lokaler Ueberbeanspruchung. Bei den meisten Balken riss die Längsbewehrung unmittelbar neben den Schweissstellen, welche die grossen Stabdurchmesser mit den kleinen verbanden. Das Reissen der Bewehrung äusserte sich durch einen Knall mit gleichzeitig starkem Abfall der Last. Beim Balken.5 ${ }_{4}$ erfolgte der Bruch durch Zerstärung der Krafteinleitungszone. Der Trägeroberflansch stauchte dabei infolge grosser Druckbeanspruchung und starker konzentrierter Krümmung.

\section{Laststellung II}

Die Belastung in Laststellung I, die im Schubbereich I zum Bruch führte, überschritt im Schubbereich II teilweise den Gebrauchszustand.

Die erste und zweite Belastungsphase der Laststellung I hatten deshalb eine geringe Bedeutung.

\section{Dritte Belastungsphase:}

Bei den Balken $S_{1}$ bis $S_{5}$ wurde in dieser Phase - bei konstanter Querbiegung - die Querkraft wiederum bis zum Bruch gesteigert. Die Steifigkeit blieb praktisch bis zum Bruch konstant; es bildete sich kein ausgeprägtes Fliessplateau aus. Nach relativ kleinen Verformungen brach der Widerstand der Balken rasch zusammen. Der Bruch kündigte sich durch starke Rissebildung und Abplatzen des Betons an der Oberfläche an. Diese Anzeichen eines typischen Querkraftsprödbruchs konnten vor allem bei den Balken $S_{1}$, $\mathrm{S}_{2}$ und $\mathrm{S}_{4}$ beobachtet werden.

Der Balkenversuch $S_{6}$ bildete bezüglich des generellen Belastungsablaufes der dritten Phase eine Ausnahme. Im Gegensatz zu allen anderen Versuchen wurde hier bei der Querkraft des Gebrauchszustandes die Querbiegung bis zum Bruch gesteigert.

Besonders interessant war in diesem Falle die Feststellung, dass sich das Querbiegemoment beträchtlich über das Bruchmoment der reinen Plattenbiegung steigern liess (ca. 30\%). Dies ist darauf zurückzuführen, dass einerseits die auf die Querbiegedruckzone verlagerten Querkraftdruckdiagonalen ein entlastendes Querbiegemoment erzeugten und andererseits die Trägerflansche einen Teil der Querbiegung über Torsion auf die im End- und Zwischenbereich verstärkten Stegzonen abgeben konnten.

\subsection{Beanspruchung der Bewehrung}

Die Dehnungen der Bewehrung wurden mit Setzdehnungsmessern der Basis $20 \mathrm{~cm}$ gemessen. Die Messstellen für die Bügel befanden sich beidseits des Steges, diejenige der Längsbewehrung in Flanschmitte an der Balkenunterseite.

\subsubsection{Belastungs-Dehnungs-Diagramme}

\section{Laststellung I}

In den Bildern 16 bis 21 sind die Belastungs-Dehnungs-Diagramme der Bügel- und Längsbewehrung aufgezeichnet. Aus diesen kurven ist, insbesondere beim Versuch $S_{1}$, die 
Querkraftumlagerung deutlich ersichtlich. Bei Fliessbeginn der Bügel auf der Zugseite übernahmen die Bügel der Druckseite den gesamten weiteren Lastzuwachs. Die Traglast wurde erst erreicht als auch die Bügel auf der Druckseite flossen.

\section{Laststellung II}

Die Bilder 22 bis 26 zeigen analog den Belastungs-Dehnungs-Verlauf in der Laststellung II. Die erste aufgezeichnete Laststufe entspricht der Vordehnung aus Laststellung I. Der Kurvenverlauf bei Laststellung II zeigt, dass vor dem Erreichen der Traglast das Fliessplateau nicht so ausgeprägt war wie bei Laststellung I. Obwohl die Mehrzahl der Bügel auf der Druckseite die Fliessbeanspruchung noch nicht erreicht hatten, liess sich die Querkraft nicht mehr weiter steigern, d.h. die Bewehrung war im Gegensatz zur Laststellung I nicht massgebend für die Traglast. Vor allem die Balken $S_{1}, S_{2}$ und $S_{4}$ wiesen eine eindeutige Ueberarmierung auf.

In den Bildern 27 bis 38 ist für die massgebenden Laststufen der Verlauf der Dehnungen der Bügel- und Längsbewehrung längs des Versuchsträgers dargestellt. Diese Bilder zeigen die beträchtliche Länge des Fliessbereiches (Länge des Schubmechanismus), der durch die sorgfältige Abstufung der Längsbewehrung erreicht werden konnte.

In den Bildern 66 bis 68 sind die gemittelten Dehnungen der Bügel- und Längsbewehrung (Mittelwerte aus den in den Bildern 16 bis 26 angegebenen Messstellen) der verschiedenen Versuche und Laststellungen in Funktion von $Q / Q_{U}^{T h}$ aufgezeichnet. Die Bruchquerkraft $Q_{u}^{T h}$ entspricht dabei der nach dem Fachwerkmodell ermittelten Schubtragfähigkeit bei reiner Längsbeanspruchung.

Beim direkten Vergleich der Versuche in Laststellung I fällt der Träger $S_{3}$ (Bild 66) dadurch auf, dass seine Bügel eine grössere Beanspruchung aufwiesen als diejenigen der anderen Versuchsträger, obwohl die Querbiegung nur unwesentlich grösser war. Dieses Verhalten ist aus der mit flacher Diagonalenneigung ( $\operatorname{tg} \alpha<0.5$ ) durchgeführten Bemessung des Balkens $\mathrm{S}_{3}$ zu erklären. Die Schubbereiche der. Laststellung II zeigen ähnlich starke Bügelbeanspruchungen, da die Bemessung, ausser beim Balken $S_{2}$, ebenfalls mit flacher Diagonalenneigung durchgeführt wurde.

\subsubsection{Beanspruchung der Bewehrung im Gebrauchszustand}

Die Beanspruchung der Bewehrung im Gebrauchszustand ist aus den Bildern 69 bis 74 ersichtlich. Die Werte $Q_{z u l}$ und $m_{z u l}$ wurden, wie bereits erwähnt, aus der theoretischen Schubbruchquerkraft $Q_{u}^{T h}$ und dem Querbiegemoment $m_{u}^{T h}$ bei reiner Längs- bzw. Querbeanspruchung bestimmt (Abschnitt 2.3.3). $\varepsilon_{z u l}$ ist dabei die der zulässigen Stahlspannung entsprechende Dehnung (SIA-Norm).

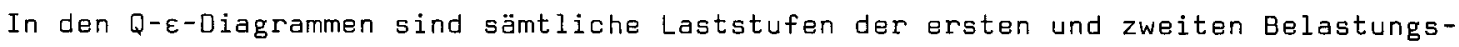
phase, in den $m-\varepsilon-\square i a g r a m m e n$ dagegen nur das Dehnungsverhalten der Bügel bei annähernd konstanter Querkraft aufgezeichnet. Der Spannungszuwachs der Bügel auf der Zug- und Druckseite entspricht im wesentlichen dem theoretischen Verhalten bei Biegung mit Normalkraft. Die Grösse des Spannungszuwachses bzw. das daraus resultierende Querbiegemoment ist jedoch wesentlich kleiner als das effektiv aufgebrachte Querbiegemoment. Dies lässt sich damit erklären, dass sich die Querkraft bei einer Steigerung der Querbiegung in die ungerissene Stegdruckzone verlagert. Die exzentrisch wirkende Schubkraft bzw. die exzantrisch wirkenden Druckdiagonalen haben aber dadurch ein der Querbiegung entgegengeseztes Moment zur Folge. Die Querkraftverlagerung wird nicht 
durch die Stahldehnung auf der Zugseite veranlasst - die Bügelbeanspruchung befindet sich noch im elastischen Bereich - , sondern allein dadurch, dass sich die QuerkraftDruckstreben nur auf der ungerissenen Druckseite der Stege ausbilden können.

Die Querkraftumlagerung im Gebrauchszustand, d.h. im elastischen Bereich, ist von entscheidender Bedeutung. Sie bewirkt, dass die Bügeldehnung auf der Zugseite, wie aus den $Q-\varepsilon-D i a g r a m m e n$ ersichtlich ist, bei voller Ueberlagerung von $Q_{z u l}$ und $\mathrm{m}_{z u l}$ die zulässige Dehnung nicht wesentlich übersteigt.

\section{2 .3 Bruch infolge Querbiegung}

Die Bilder 75 und 76 enthalten die in Laststellung II gemessenen Bügeldehnungen des Balkens $S_{6}$, dessen Belastungsablauf von den vorhergehenden Versuchen abwich (Bruch durch Steigerung der Querbiegung bei konstanter Querkraft $Q_{z u l}$ J. Die Messungen zeigten deutlich, dass die Querbiegung nicht allein durch die Bügel, sondern zu einem beträchtlichen Teil auch durch die exzentrisch wirkende Querkraft aufgenommen wurde. Bei $m_{u}^{T h}$ bildete sich deshalb noch kein Fliessplateau aus. Vor dem Erreichen des Querbiegebruchmomentes wurde allerdings auch ein Teil der im Schubbereich aufgebrachten Querbiegung über Flanschtorsion auf die stärker bemessenen Zonen der Stege übertragen. Diese Lastumlagerung in der Endphase der Laststeigerung erfolgte jedoch erst bei starkem Steifigkeitsabfall, und sie betrug dem Torsionswiderstand des Zugflansches entsprechend höchstens $0.35 \mathrm{mt}$, d.h. ca. $10 \%$ der im geprüften Schubbereich aufgebrachten Querbiegung.

\subsection{Beanspruchung des Betons}

\subsubsection{Druckflansch}

Die Betonstauchungen wurden mit Setzdehnungsmessern der Basis $20 \mathrm{~cm}$ an der Oberseite des Druckflansches gemessen. Der Verlauf der Betonstauchungen längs der Balken ist in den Bildern 39 bis 42 dargestellt. Die Werte entsprechen den Erwartungen, weisen jedoch zum Teil erhebliche Streuungen auf.

Interessant war das starke lokale Anwachsen der Stauchungen nach Fliessbeginn der Längsbewehrung. Dieser stauchungszuwachs lässt sich mit der starken lokalen Flanschbiegung infolge Längsbeanspruchung erklären. Hier begann auch die beim Bruch beobachtete Zerstörung des Druckflansches.

\subsubsection{Betondruckdiagonalen im Steg}

Aufgrund des komplizierten Risseverhaltens (Horizontal- und Schrägrisse) wurde auf eine Messung der Betondruckstauchungen im Steg verzichtet. Ein erst nach der Rissebildung und unter einem Winkel von $45^{\circ}$ aufgeklebter Dehnungsmessstreifen diente zur Ueberwachung der Betonbeanspruchungen. Die damit ermittelten Deformationen erreichten in Laststellung II Werte in der Grössenordnung der Bruchstauchungen. Die hohe Beanspruchung des Betons zeigte sich auch durch Abplatzungen an der Betonoberfläche. 
4. 4 Verformungen

4.4.1 Schiebungen

Die Schiebungen $\gamma$ in den Schubbereichen wurden mit Setzdehnungsmessern durch Ausmessen der Abschnittseiten bestimmt. [2] enthält eine eingehende Beschreibung der Berechnung der Winkeländerungen in den vier Ecken des quadratischen Messabschnittes und der sich daraus ergebenden Schiebungen.

Die Schiebungen in Abhängigkeit der Querkraft sind in den Bildern 60 bis 65 aufgetragen und zeigen deutlich die Länge des Schubmechanismus. Aus den auf der Druck- und Zugseite ermittelten Schiebungen ging hervor, dass die Verformungen auf beiden Seiten übereinstimmten, obwohl die Bügeldehnungen erhebliche Unterschiede aufwiesen. Dies lässt auf eine gegenseitige Unterstützung der beiden Steghälften in der Aufnahme der Querkraft schliessen. Die starke Druckseite hilft der durch Querbiegung geschwächten Zugseite und ermöglicht bei unterarmierten Stegen eine volle Interaktion zwischen Schub- und Querbiegung.

\subsubsection{Durchbiegungen}

Die Durchbiegungen wurden an der Balkenunterseite gemessen und sind in Funktion der Querkraft in den Bildern 43 bis 47 und 58 aufgetragen. Aus diesen Kurven ist das Tragverhalten und insbesondere der Unterschied zwischen dem duktilen und spröden Bruchverhalten deutlich erkennbar. Die Bilder 48 bis 57 und 59 zeigen den Verlauf der Durchbiegungen längs der Balken. Auffallend dabei ist die asymmetrische Trägerverformung, die sich aus den ungleich stark bewehrten Schubbereichen ergibt.

\subsection{Risseverhalten}

\section{$\underline{4.5 .1 \text { Risseverlauf }}$}

Die Bilder 77 und 78 vermitteln einen Ueberblick über das Risseverhalten der Versuchsträger in verschiedenen Laststufen. Der Risseverlauf wurde mit einem Filzschreiber nachgezogen und die Rissweiten in $V 100 \mathrm{~mm}$ angegeben. Die ersten Risse im Schubbereich entstanden unter einer Neigung von $45^{\circ}$, wobei die Beanspruchung bei 50 bis $70 \%$ der Gebrauchslast lag. Infolge Querbiegung bildeten sich auf der gesamten Steghöhe Horizontalrisse. Nach den Lastwechseln im Gebrauchszustand entstand bei zunehmender Querkraft im wesentlichen folgendes Rissebild:

Zugseite:

Die neu auftretenden Risse waren sehr flach geneigt. Die Horizontalrisse blieben in ihrer Weite konstant.

Druckseite:

Die relativ wenigen Schubrisse wiesen durchwegs eine Neigung von mehr als $45^{\circ}$ auf. Die Steigerung der Querkraft änderte das Rissebild nur unwesentlich. Allerdings nahm bei Fliessbeginn der "Druckbügel" die Anzahl und Grösse der Schubrisse auf der Druckseite stark zu. 


\subsubsection{Rissweiten}

Die Rissweiten wurden mit einem Rissemassstab und einer Genauigkeit von ca. 5/100 mm gemessen. In den Bildern 79 und 80 sind die Werte sämtlicher Versuchsbalken aufgetragen. Die Beanspruchung in der mit einem Doppelkreis bezeichneten Laststufe entspricht der Ueberlagerung der zulässigen Querkraft mit der zulässigen Querbiegung.

Aus Bild 79 (Laststellung I) ist ersichtlich, dass der Träger $S_{3}$ eine verhältnismässig. grosse Rissweite aufwies. Ursache dafür war die flache Diagonalenneigung, mit der die Bemessung durchgeführt wurde. Die Stege der Balken $S_{4}$ und $S_{5}$, die besonders kleine Rissweiten zeigten, waren asymmetrisch bewehrt. Das Verhältnis der Bügelbewehrung auf der Druckseite zu jener der Zugseite betrug 0.6, resp. 0.5. In Laststellung II wiesen die Balken grössere Risse auf. Dies ist wiederum darauf zurückzuführen, dass in diesem Schubbereich alle Balken, ausser $S_{2}$, mit flacher Diagonalenneigung bemessen wurden, und dass die Vorbelastung infolge Laststellung I bereits über dem Gebrauchszustand lag.

4.6 Vergleich zwischen Norm und Versuchen

4.6.1 Vergleich der experimentellen Bruchlast mit der duktilen Schubtragfähigkeit bei reiner Längsbeanspruchung

In den Bildern $86 a$ und $86 b$ sind die experimentell ermittelten Schubtraglasten $Q_{u}^{E x}$ in einem bezogenen Querkraft-Querbiege-Interaktionsdiagramm dargestellt. Bezugsgrössen sind die theoretisch bestimmten Traglasten für Querkraft und Querbiegung bei reiner Längs - und Querbeanspruchung.

Bild 84a zeigt die Versuche in Laststellung I. Daraus ist ersichtlich, dass bei unterarmierten Trägern auch bei grosser Querbiegung die volle, der gesamten Stegbewehrung entsprechende Schubtragfähigkeit erreicht wird. Ein grosses Querbiegemoment bewirkt lediglich einen früheren Fliessbeginn der Bügel auf der Zugseite. Das etwas ungünstigere Risseverhalten gegenüber der reinen Längsbeanspruchung lässt sich bei Schub und Querbiegung durch asymmetrische Stegbewehrung (Verlagerung der Stegbewehrung auf die Zugseitel verbessern; dies ist beim Versuch $S_{5}$ deutich erkennbar.

In Bild 84b sind die Versuchsergebnisse der Laststellung II aufgetragen. Die Bruchlasten lagen durchwegs deutlich unter der der Bewehrung entsprechenden Traglast. Die Bügel auf der Zugseite und die Längsbewehrung kamen zwar auch bei diesen Versuchen zum Fliessen, die Bügel auf der Druckseite dagegen nicht oder nur sehr beschränkt. Daraus kann geschlossen werden, dass die Bruchursache nicht die Ueberbeanspruchung der Bewehrung, sondern ein Versagen der Betondruckzone war.

4.6.2 Vergleich der experimentellen Bruchlast mit der Schubtragfähigkeit überarmierter Querschnitte (obere Schubspannungsgrenze bei reiner Längsbeanspruchung)

Der Vergleich der experimentell festgestellten nominellen Schubspannung mit der in der Norm vorgesehenen oberen Schubspannungsgrenze bei reiner Längsbeanspruchung gibt Aufschluss über das Sprödbruchverhalten bei kombinierter Beanspruchung durch Schub und Querbiegung.

In der Laststellung I lag die nominelle Schubspannung bei 40 bis $50 \mathrm{kE} / \mathrm{cm}^{2}$ oder, verglichen mit der rechnerischen Schubspannung, bei $3.5 \cdot \tau_{r}$ bis $4 \cdot \tau_{r}$. Alle diese Versuche 
zeigten eindeutig ein duktiles Bruchverhalten.

Bei den Versuchen in Laststellung II lag die Schubbeanspruchung bedeutend höher, und alle Versuche wiesen Anzeichen eines Sprödbruches auf: oberflächliches Abplatzen des Betons, kein ausgeprägtes Fliessplateau, Bruch vor dem Erreichen der duktilen Traglast.

Aus Bild 85 ist die beim Bruch festgestellte Schubspannung ersichtlich. Sie liegt bei allen Versuchen unter dem Normwert. Da die obere Schubspannungsgrenze $6 \cdot \tau_{\Gamma}$ (bei reiner Längsbeanspruchung) vorsichtig angesetzt ist, muss der stark verminderten Sprödbruchsicherheit bei Schub mit Querbiegung umso mehr Beachtung geschenkt werden. Das Bild 87 zeigt die nominelle Schubspannung der Träger $S_{1}$ bis $S_{5}$ in Laststellung II im Vergleich zu den Versuchen [3], [4], [5] bei reiner Längsbeanspruchung. 


\subsection{Dimensionierung der Bewehrung}

Die durchgeführten Versuche haben eindeutig gezeigt, dass eine Leberlagerung der Bewehrungsquerschnitte für Schub und Querbiegung nicht notwendig ist. Es genügt, wenn die Bewehrung für die massgebende der beiden Beanspruchungen bemessen wird. Die volle Interaktion zwischen Schub und Querbiegung bei duktilem Verhalten lässt sich darauf zurückführen, dass sich die Lage der Querkraft im Stegquerschnitt optimal einstellt. Die Querkraftverlagerung ist allerdings nur dann möglich, wenn der Gleichgewichtszustand des Trägers bei exzentrischer Querkraft gewährleistet ist. Bei den Versuchsträgern verursachte die Querkraftverlagerung Torsion, die vorwiegend durch Flanschbiegung aufgenommen wurde. Bei den im Brückenbau üblichen Querschnitten ergibt sich infolge der Querkraftverlagerung keine Störung des Gleichgewichtes.

Zur Verbesserung des Risseverhaltens im Gebrauchszustand sind bei Schub und Querbiegung folgende Massnahmen zu treffen:

- Bei symmetrischer Stegbewehrung sollte die der Bemessung zugrunde gelegte Diagonalenneigung nicht flacher als $45^{\circ}$ sein.

- Eine flachere Diagonalenneigung sollte nur bei asymmetrischer Bügelbewehrung in Betracht gezogen werden (höhere Stegbewehrung auf der Zugseite).

\subsection{Betonabmessungen}

Die Versuche in Laststellung II zeigten eindeutig, dass die Sprödbruchsicherheit schubbeansprichter Träger durch Querbiegung stark reduziert wird. Die Ursache hiefür liegt wiederum bei der Verlagerung der Querkraft in die Biegedruckzone; die Superposition der Betonbeanspruchungen infolge Querkraft (Druckdiagonalen) und Querbiegung führte zu einem vorzeitigen Versagen der Druckzone des Trägersteges.

Eine genaue Bestimmung der oberen Schubspannungsgrenze in Funktion des Querbiegemomentes ist aufgrund der vorliegenden Versuche nicht möglich. Es zeigt sich jedoch, dass mit dem Erreichen des Rissemomentes infolge Querbiegung die obere Schubspannungsgrenze stark abfällt; unter Berücksichtigung einer analogen Sprödbruchsicherheit wie bei reiner Längsbeanspruchung sollte sie bei ca. $4 \cdot \tau_{r}$ angesetzt werden. Bei grösseren Querbiegemomenten ist keine weitere Reduktion der Schubspannungsgrenze angezeigt, erst kurz vor dem Erreichen des Sprödbruchmomentes infolge Querbiegung ist ein vollständiges Absinken der Schubspannungsgrenze auf null zu erwarten. 
Das am Institut für Baustatik und Konstruktion der Eidgenössischen Technischen Hochschule Zürich durchgeführte Forschungsprojekt "Schub bei Querbiegung" umfasste eingehende Untersuchungen über das Verhalten schubbeanspruchter Träger, deren Stege auf ihrer gesamten Länge durch eine konstante Querbiegung beansprucht werden. Diese Untersuchungen basierten auf der Prüfung von sechs I-fürmigen Stahlbetonbalken in je zwei Laststellungen.

Das Ziel des Forschungsprojektes bestand in der Abklärung der beiden folgenden Probleme:

- Bemessung der Stegbewehrung,

- Ermittlung der oberen Schubspannungsgrenze.

Die Hauptparameter der Versuchsträger bestanden einerseits aus den Schubkennwerten (Bügelbewehrung, Längsbewehrung und Diagonalenneigung) und andererseits aus den Querbiegekennwerten (Querbiegebeanspruchung, Verhältnis der Bügelbewehrung auf der Zugund Druckseite der Stege).

Die Parameter waren so gewählt, dass die Traglast der Versuchsbalken in Laststellung I durch Ueberbeanspruchung der Bewehrung (duktiles Verhalten) und in Laststellung II durch Versagen der Betondruckzone (Sprödbruch) erreicht wurde.

Die Ergebnisse der Versuche lassen sich wie folgt zusammenfassen:

Bei duktilem Verhalten wird die Schubtraglast durch Querbiegung nicht beeinflusst, sofern eine Verlagerung der Querkraft in die Stegdruckzone ohne Störung des Gleichgewichtes möglich ist. Es besteht somit eine volle Interaktion zwischen Querkraft und Querbiegung, d.h. die Stegbewehrung muss nur für die massgebende der beiden Beanspruchungen bemessen werden. Im Gebrauchszustand ist die Beanspruchung der Bewehrung auf der Stegzugseite infolge der Querkraftverlagerung in die Stegdruckzone wesentlich kleiner als bei voller Veberlagerung von Schub und Querbiegung. Das Rissebild lässt sich durch eine Diagonalenneigung von ca. $45^{\circ}$ (vgl. Fachwerkmodell der Richtlinie 34 der SIA Norm 162) oder durch asymmetrische Anordnung der Stegbewehrung günstig beeinflussen.

Bei sprödem Tragverhalten hat eine das Rissemoment übersteigende Querbiegung einen starken Abfall der Schubtraglast zur Folge. Genaue Werte für die obere Schubspannungsgrenze lassen sich aufgrund der relativ kleinen Versuchszahl nicht angeben, da bei einem Sprödbruch erhebliche Streuungen auftreten. Die nominelle Schubspannung bei Schub mit Querbiegung sollte jedoch $60 \%$ der für reinen Schub festgelegten Schubspannungsgrenze nicht überschreiten. 
Dans le cadre de son programme de recherches "cisaillement avec flexion latérale", l'Institut de Statique et de Construction de l'Ecole Polytechnique Fédérale de Zürich s'est livré à une étude approfondie du comportement de poutres soumises au cisaillement, dont les âmes sont sollicitées sur toute leur longueur par une flexion latérale constante. Ces recherches s'appuient sur les essais effectués sur six poutres en bétón armé à section en I, selon deux schémas de charge.

Ce programme de recherches avait pour but d'éclaircir les deux points suivants:

- dimensionnement de l'armature des âmes,

- définition de la limite supérieure des contraintes tangentielles.

Les poutres soumises aux essais se différenciaient d'une part par des paramètres intéressant le cisaillement (armatures en long, étriers, inclinaison des diagonales) et d'autre part par des paramètres intéressant la flexion latérale (niveau de sollicitation, rapport des surfaces des étriers entre la face tendue et la face comprimée).

On a choisi les paramètres de telle sorte que les poutres soumises au schéma de charge I atteignent leur charge ultime par fluage des armatures (comportement ductil), tandis que le schéma de charge II provoquait une rupture de la zone comprimée du béton (rupture fragile).

On peut résumer les résultats obtenus de la façon suivante: Dans le cas du comportement ductil, la charge ultime de cisaillement n'est pas influencée par la flexion latérale, tant que les efforts tranchants peuvent se redistribuer dans la zone comprimée de l'âme sans influencer l'état d'équilibre. Il y a donc interaction complète entre l'effort tranchant et la flexion latérale, c'est-à-dire qu'on ne dimensionne l'armature de l'âme que pour le type de sollicitation déterminant. Dans l'état de service, la sollicitation des armatures dans la zone tendue de l'âme est - du fait de la redistribution des efforts tranchants dans la zone comprimée de l'âme - nettement inférieure.

On peut obtenir une répartition plus favorable des fissures grâce à une inclinaison denviron $45^{\circ}$ des diagonales (voir le modéle du treillis de la directive 34 de la norme SIA 162) ou grâce à un ferraillage asymétrique de l'âme.

Dans le cas de rupture fragile, toute flexion latérale excédant le moment de fissuration entraine une forte diminution de la charge ultime de cisaillement. En se basant sur un nombre restreint d'essais, on ne peut pas encore indiquer les valeurs exactes de la limite supérieure des contraintes tangentielles, les résultats d'essais de rupture fragile laissant apparaitre une assez grande dispersion. La contrainte tangentielle nominale en cas de cisaillement avec flexion latérale ne devrait toutefois pas dépasser $60 \%$ de la limite supérieure des contraintes tangentielles fixée pour le cisaillement pur. 
The research project "Shear with Transverse Bending", performed at the Institute of Structural Engineering. Swiss Federal Institute of Technology (ETH) Zurich, covered detailed studies on the behavior of beams of which the webs were loaded under combined shear and transverse bending. The transverse bending was constant along the beams. The studies were based on a test series of six I-shaped reinforced concrete beams. Each beam was tested with two different load arrangements.

The aim of the research project was to analyse the following two problems:

- Design of the shear reinforcement

- Determination of the upper limit of shear stresses

The principal parameters were composed of characteristic values of shear (stirrup reinforcement, longitudinal reinforcement and inclination of the concrete struts) and of characteristic values of transverse bending (intensity of transverse bending, ratio of the stirrup reinforcement in the tension zone and compression zone of the web).

The parameters were chosen in such a way that the ultimate load of the test beams was reached after yielding of the reinforcement (ductile behavior) with the first load arrangement and after crushing of the concrete (brittle failure) with the second load arrangement.

The test results may be summarized as follows:

If the beam shows a ductile behavior the ultimate shear force is not influenced by transverse bending as far as the shear force may be shifted towards the compression zone of the web without distrubing equilibrium. A full interaction between shear force and transverse bending can be observed; i.e. the web reinforcement ras to be designed only for the dominant of the two loadings. Under service load the stirrup stresses in the tension zone of the web are essentially lower than expected according to the superposition of shear and transverse bending because the shear force is shifted towards the compression zone. The crack pattern may be influenced favorably with struts inclined at about $45^{\circ}$ or with an asymmetric web reinforcement.

If the beam shows a brittle failure the ultimate shear force decreases substantially as far as the transverse bending moment exceeds the cracking moment. The rather small number of tests does not allow the exact determination of the upper limit of shear stresses because a large variation of the test results is possible. However, the calculated shear stresses of transverse bent webs should not exceed $60 \%$ of the upper limit fixed for pure shear. 
Dieser Versuchsbericht wurde im Rahmen des Forschungsprojektes "Schub bei Querbiegung" am Institut für Baustatik und Konstruktion der Eidgenössischen Technischen Hochschule Zürich (ETHZ) ausgearbeitet. Für die grosszügige finanzielle Unterstützung diese Projektes möchte ich dem

$$
\text { Eidg. Amt für Strassen- und Flussbau, Bern }
$$

und dem

$$
\text { Kant. Autobahnamt Bern }
$$

aufrichtig danken.

Der experimentelle Teil der Untersuchungen wurde an der Eidgenössischen Materialprüfungs - und Versuchsanstalt (EMPA) in Dübendorf durchgeführt. Für das Entgegenkommen danke ich den Herren Prof. Dr. Th. Erismann, Direktionspräsident der EMPA sowie Dr. M. Ladner, Vorsteher der Abteilung Massivbau. Ausserdem gilt mein Dank der Monteforno Stahl- und Walzwerke $A G$ in Bodio, die sämtliche Bewehrungsstähle für die Versuchsbalken zur Verfügung stellte.

Bei der Versuchsdurchführung haben die Herren $K$. Bucher und $C$. Tiniç mitgewirkt. Herr M. Baumann, dipl. Bauing., befasste sich mit den messtechnischen Problemen der erweiterten Mess- und Datenerfassungsanlage. Für die Gestaltung des Berichtes und die Zeichnungen war Herr G. Göseli zuständig. Für diese Mitarbeit möchte ich den Genannten wie auch den andern an der Versuchsdurchführung Beteiligten bestens danken. 
[1] Lampert P., Wegmüller A., Thürlimann B.: "Einfluss der Dehngeschwindigkeit auf Festigkeitswerte von Armierungsstählen", Institut für Baustatik, ETH * Zürich, Bericht Nr. 10, April 1967.

[2] Lampert P., Thürlimann B.: "Torsiansversuche an Stahlbetonbalken", Institut für Baustatik, ETH Zürich, Bericht Nr. 6506-2, Juni 1968. Birkhäuser Verlag Basel und Stuttgart.

[3] Leonhardt F., Walther R.: "Versuche an Plattenbalken mit hoher Schubbeanspruchung", Deutscher Ausschuss für Stahlbeton, Heft Nr. 152, 1962.

[4] Robinson J.R.: "Essais à l'effort tranchant de poutres a âme mince en béton armé", Annales des ponts et chaussées, Mars-Avril 1961.

[5] Slater W.A., Lord A.R., Zippradt R.R.: "Shear Tests of Reinforced Concrete Beams", Technologic Papers of the Bureau of Standards, No. 314, 1926. 
BEZEICHNUNGEN

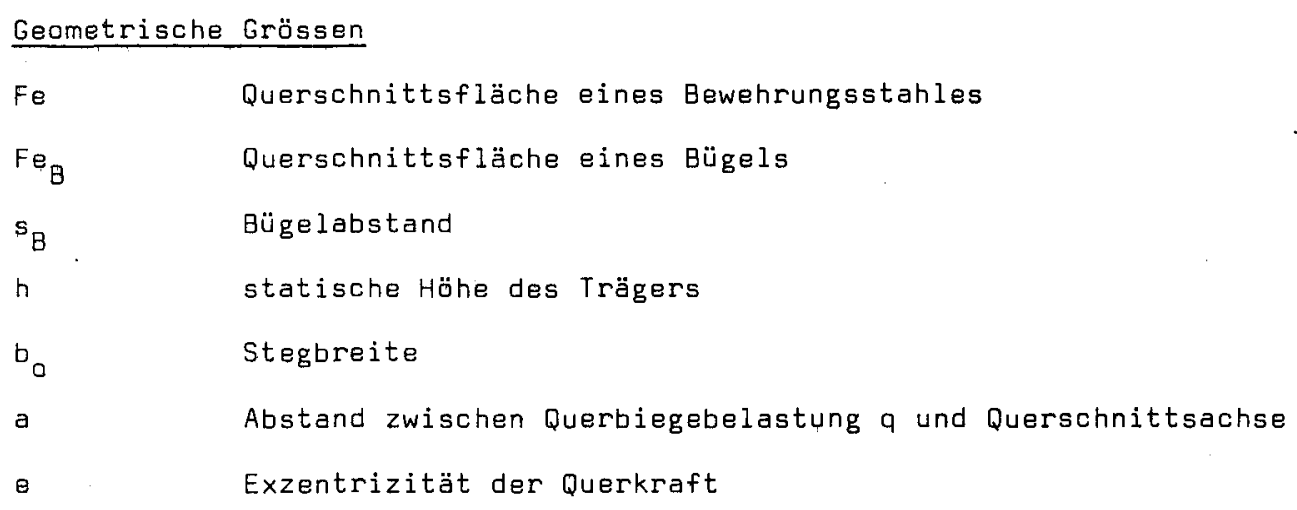

\section{Kraftgrössen}

\begin{tabular}{|c|c|}
\hline$m_{q}$ & vorhandenes Querbiegemoment \\
\hline$m_{z \cup 1}$ & zulässiges Querbiegemoment \\
\hline$m_{u}^{T h}$ & theoretisches Querbiegebruchmoment \\
\hline Q & vorhandene Querkraft \\
\hline$Q_{z \cup 1}$ & zulässige Querkraft \\
\hline & theoretische Bruchquerkraft \\
\hline$Q_{u}^{E x}$ & experimentelie Bruchquerkraft \\
\hline$Q_{f Z}$ & Querkraft bei Fliessbeginn der Zugbügel \\
\hline$Q_{f D}$ & Querkraft bei Fliessbeginn der Druckbügel \\
\hline$Q_{f L}$ & Querkraft bei Fliessbeginn der Längsbewehrung \\
\hline$Q_{A}$ & Querkraft bei Beginn der Laststufe \\
\hline$Q_{E}$ & Querkraft am Ende der Laststufe \\
\hline$Q_{b}$ & Schubwiderstandsanteil \\
\hline M & vorhandenes Biegemoment \\
\hline$z_{U}$ & Zugkraft im Untergurt \\
\hline B & Zugkraft-eines Bügels \\
\hline$P$ & Kolbenkraft der Hauptpressen \\
\hline $\mathrm{q}$ & Kolbenkraft der Querbiege-Zugpressen \\
\hline $\mathrm{H}_{0}, \mathrm{H}_{U}$ & seitliche Festhaltekräfte \\
\hline A & Auflagerkräfte \\
\hline & Festhaltekraft in Längsrichtung \\
\hline
\end{tabular}

\section{Festigkeitswerte, Spannungen}

$\begin{array}{ll}\tau^{*} & \text { nominelle Schubspannung } \\ \tau_{Q}^{*} & \text { nominelle Schubspannung infolge Querkraft } \\ \tau^{*}\left(E_{Q_{u}}^{E x}\right. & \text { nominelle Schubspannung bei Bruch }\end{array}$




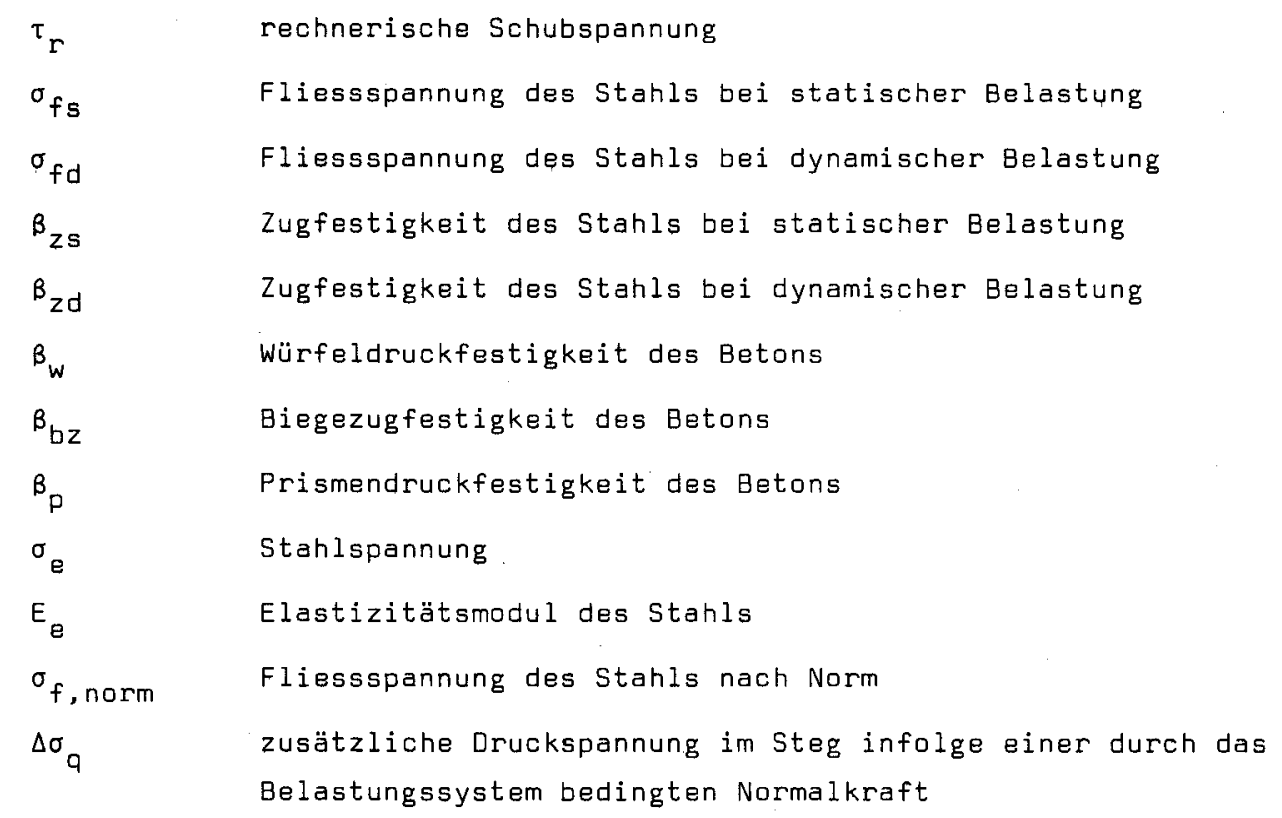

\section{Verformungen}

$\begin{array}{ll}\varepsilon_{\text {längs }} & \text { Längsdehnung } \\ \varepsilon_{\text {quer }} & \text { Querdehnung } \\ \varepsilon_{\text {ev }} & \text { Stahldehnung bei Beginn der Verfestigung } \\ \lambda_{g l} & \text { Gleichmassdehnung } \\ \dot{\varepsilon} & \text { Dehnungsgeschwindigkeit bei Zugversuch des Stahls } \\ \varepsilon_{e} & \text { Stahldehnung } \\ \varepsilon_{f} & \text { Stahldehnung bei Fliessbeginn } \\ \varepsilon_{z u l} & \text { Stahldehnung bei zulässiger Stahlspannung } \\ \delta & \text { Durchbiegung } \\ \gamma & \text { Schiebung } \\ r & \text { Rissweite } \\ k & \text { Krümmung }\end{array}$

\section{Dimensionslose Parameter}

$\alpha \quad$ Neigung der Betondruckdiagonalen

$\mu_{Z} \quad$ Bügelbewehrungsgehalt auf der Zugseite

$\mu_{0} \quad$ Bügelbewehrungsgehalt auf der Druckseite

$\mu_{\theta} \quad$ rechnerischer Bügelbewehrungsgehalt

seff effektiver Bruchsicherheitsfaktor

\section{Allgemeine Bezeichnungen}

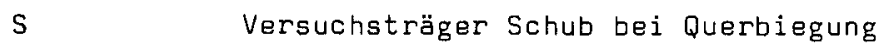

I, I Laststellung, Schubbereich 
LS

$1,2 \ldots$

\section{Indizes}

b

$u$

Th

Ex

Z

D

B

0

U

A

E

$f$

d

5
Laststellung

Phasen, Laststufen, Versuchsträger

Beton

Stahl

Bruch

theoretisch ermittelter Wert

experimentell ermittelter Wert

Zugseite des Steges

Druckseite des Steges

Bügel

oben

unten

Beginn der Laststufe

Ende der Laststufe

Fliessen

dynamisch

statisch 


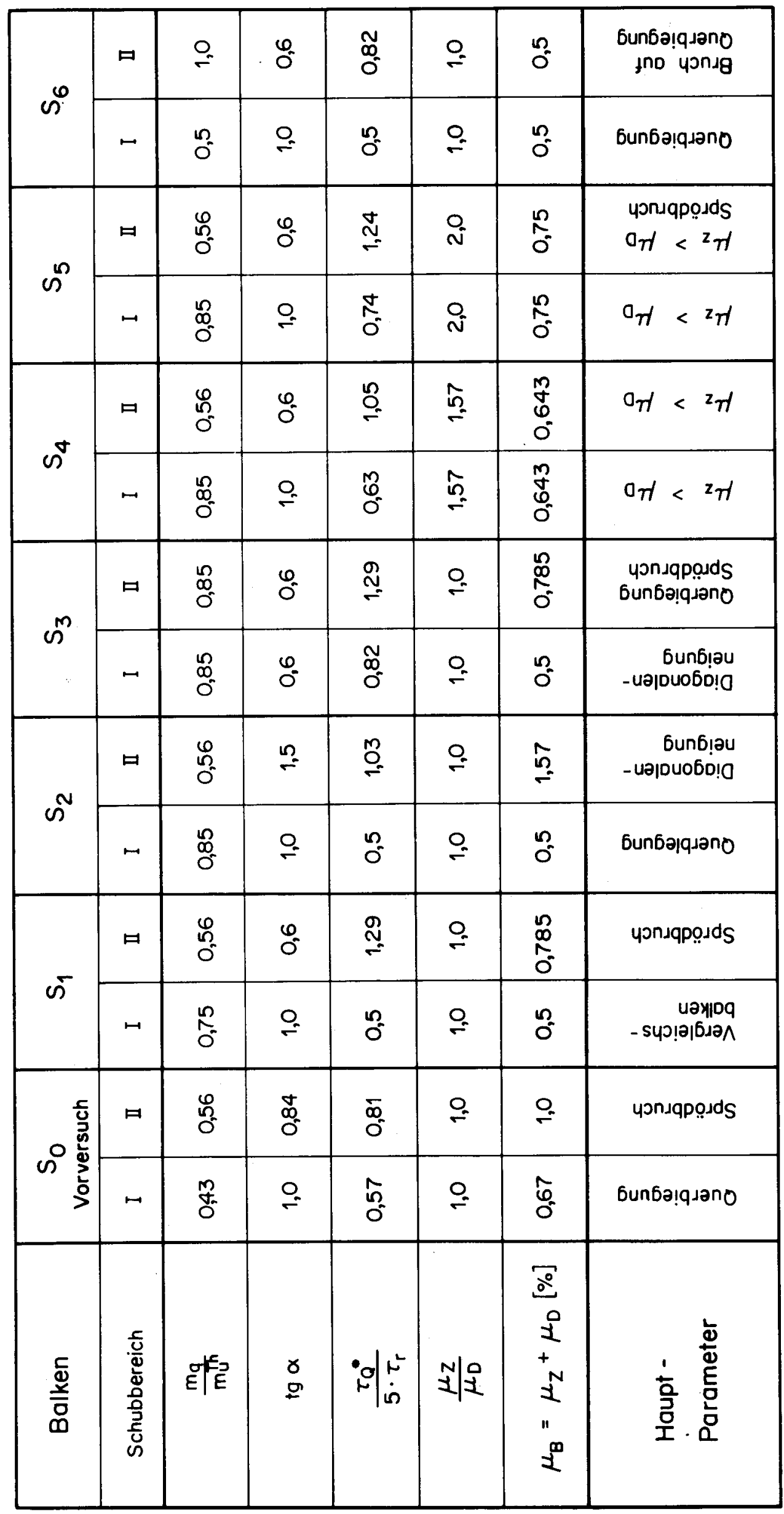

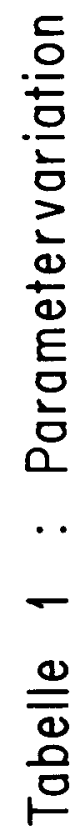




\begin{tabular}{|c|c|c|c|c|c|c|c|}
\hline $\begin{array}{c}\text { Nenn } \phi \\
\mathrm{mm}\end{array}$ & $\begin{array}{c}F_{\mathrm{e}} \\
\mathrm{cm}^{2}\end{array}$ & $\begin{array}{c}\sigma_{\mathrm{fs}} \\
t / \mathrm{cm}^{2}\end{array}$ & $\begin{array}{c}\sigma_{\mathrm{fd}} \\
\mathrm{t} / \mathrm{cm}^{2}\end{array}$ & $\begin{array}{c}\beta_{\mathrm{zs}} \\
t / \mathrm{cm}^{2}\end{array}$ & $\begin{array}{c}\beta_{\mathrm{zd}} \\
t / \mathrm{cm}^{2}\end{array}$ & $\begin{array}{c}\varepsilon_{\mathrm{ev}} \\
\%\end{array}$ & $\begin{array}{c}\lambda_{\mathrm{Gl}} \\
\%\end{array}$ \\
\hline 8 & 0,50 & 5,50 & 5,67 & 6,65 & 7,00 & 2,66 & 16 \\
10 & 0,79 & 5,41 & 5,61 & 6,66 & 7,04 & 2,66 & 16 \\
12 & 1,13 & 5,82 & 6,07 & 7,35 & 7,70 & 2,42 & 14 \\
16 & 2,01 & 5,51 & 5,71 & 6,85 & 7,16 & 2,62 & 13 \\
20 & 3,14 & 5,11 & 5,32 & 6,70 & 7,07 & 2,18 & 13 \\
\hline
\end{tabular}

Tabelle 2: Festigkeitswerte des BOX-Stahles

\begin{tabular}{|l|c|c|c|c|c|c|c|c|c|}
\hline Balken & $\begin{array}{c}\text { Sand } \\
0 \div 4 \mathrm{~mm}\end{array}$ & $\begin{array}{c}\text { Kies } \\
4 \div 8 \mathrm{~mm}\end{array}$ & $\begin{array}{c}\text { Kies } \\
8 \div 16 \mathrm{~mm}\end{array}$ & $\begin{array}{c}\text { Zuschlog- } \\
\text { stoffe } \\
\mathrm{kg} / \mathrm{m}^{3}\end{array}$ & $\begin{array}{c}\text { Zement } \\
\mathrm{PC} \\
\mathrm{kg} / \mathrm{m}^{3}\end{array}$ & $\begin{array}{c}\text { Wasser } \\
\mathrm{L} / \mathrm{m}^{3}\end{array}$ & $\begin{array}{c}\text { W/Z } \\
\text { Faktor }\end{array}$ & $\begin{array}{c}\text { Raum- } \\
\text { gewicht } \\
\mathrm{kg} / \mathrm{m}^{3}\end{array}$ & $\begin{array}{c}\text { Konsistenz } \\
\text { Verdichtungsmass }\end{array}$ \\
\hline $\mathrm{S}_{1} \div \mathrm{S}_{3}$ & $49 \%$ & $23 \%$ & $28 \%$ & 2365 & 349 & 189 & 0,54 & 2365 & $\begin{array}{c}\text { plastisch } \\
1,09\end{array}$ \\
\hline $\mathrm{S}_{4} \div \mathrm{S}_{6}$ & $49 \%$ & $23 \%$ & $28 \%$ & 2370 & 320 & 193 & 0,60 & 2370 & $\begin{array}{c}\text { plastisch } \\
1,15\end{array}$ \\
\hline
\end{tabular}

Tabelle 3: Zusammensetzung des Frischbetons

\begin{tabular}{|c|c|c|c|c|c|c|c|}
\hline Bolken & Mischung & $\begin{array}{l}\text { Anzohl } \\
\text { Proben }\end{array}$ & $\begin{array}{c}\beta_{\mathrm{w}} \\
\mathrm{kg} / \mathrm{cm}^{2}\end{array}$ & $\begin{array}{c}\beta_{\mathrm{bz}} \\
\mathrm{kg} / \mathrm{cm}^{2}\end{array}$ & $\varepsilon_{\substack{\text { längs } \\
\% \circ}}$ & $\begin{array}{c}\varepsilon_{\text {quer }} \\
\% \text { \%o }\end{array}$ & $\beta_{\mathrm{kg} / \mathrm{cm}^{2}}$ \\
\hline$S_{1}$ & $\begin{array}{l}1 \\
2\end{array}$ & $\begin{array}{l}2 \\
3\end{array}$ & $\begin{array}{l}406 \\
389\end{array}$ & $\begin{array}{l}42 \\
43\end{array}$ & $\begin{array}{l}2,5 \\
-\end{array}$ & $\begin{array}{l}1,6 \\
-\end{array}$ & $\begin{array}{c}320 \\
-\end{array}$ \\
\hline$S_{2}$ & $\begin{array}{l}1 \\
2\end{array}$ & $\begin{array}{l}2 \\
3\end{array}$ & $\begin{array}{l}433 \\
411\end{array}$ & $\begin{array}{l}37 \\
41\end{array}$ & $\begin{array}{c}2,3 \\
-\end{array}$ & $\begin{array}{l}1,1 \\
-\end{array}$ & $\begin{array}{c}315 \\
-\end{array}$ \\
\hline$S_{3}$ & $\begin{array}{l}1 \\
2\end{array}$ & $\begin{array}{l}2 \\
3\end{array}$ & $\begin{array}{l}437 \\
407\end{array}$ & $\begin{array}{l}44 \\
41\end{array}$ & $\begin{array}{c}2,5 \\
-\end{array}$ & $\frac{1,3}{-}$ & $\begin{array}{l}350 \\
-\end{array}$ \\
\hline \multicolumn{3}{|c|}{ Mittelwert } & $\begin{array}{c}412 \\
(V=4,6 \%)\end{array}$ & $\begin{array}{c}41 \\
(V=7,5 \%)\end{array}$ & 2,43 & 1,33 & 328 \\
\hline $\mathrm{S}_{4}$ & $\begin{array}{l}1 \\
2\end{array}$ & $\begin{array}{l}3 \\
2\end{array}$ & $\begin{array}{l}331 \\
338\end{array}$ & $\begin{array}{l}36 \\
33\end{array}$ & $\overline{1,86}$ & $\overline{1,07}$ & $\frac{-}{244}$ \\
\hline$S_{5}$ & $\begin{array}{l}1 \\
2\end{array}$ & $\begin{array}{l}3 \\
2\end{array}$ & $\begin{array}{l}334 \\
332\end{array}$ & $\begin{array}{l}44 \\
31\end{array}$ & $\overline{1,75}$ & $\overline{0,85}$ & $\frac{-}{267}$ \\
\hline$S_{6}$ & $\begin{array}{l}1 \\
2\end{array}$ & $\begin{array}{l}3 \\
2\end{array}$ & $\begin{array}{l}302 \\
299\end{array}$ & $\begin{array}{l}36 \\
31\end{array}$ & $\overline{1,6}$ & $\overline{0,60}$ & $\overline{214}$ \\
\hline \multicolumn{3}{|c|}{ Mittelwert } & $\begin{array}{c}323 \\
(V=5 \%)\end{array}$ & $\begin{array}{c}36 \\
(V=14 \%)\end{array}$ & 1,74 & 0,84 & 242 \\
\hline
\end{tabular}

Tabelle 4: Festigkeitswerte der Betonprismen nach 28 Tagen 


\begin{tabular}{|c|c|c|c|}
\hline$s_{1}$ & \multicolumn{2}{|c|}{ Loststellung } & \\
\hline $\begin{array}{l}\text { Last - } \\
\text { stufe }\end{array}$ & $\begin{array}{c}Q_{A} \\
t\end{array}$ & $\begin{array}{c}Q_{E} \\
t\end{array}$ & $\underset{\mathrm{mt} / \mathrm{m}^{\prime}}{\mathrm{m}_{\mathrm{q}}}$ \\
\hline 1 & 1,3 & 1,3 & 0 \\
\hline 2 & 7,4 & 6,6 & 0 \\
\hline 3 & 9,55 & 9,0 & 0 \\
\hline 4 & 9,0 & 8,8 & 0,46 \\
\hline 5 & 8,8 & 8,6 & 0,62 \\
\hline 6 & 1,3 & 1,3 & 0 \\
\hline 7 & 9,55 & 9,1 & 0 \\
\hline 8 & 9,05 & 8,9 & 0,62 \\
\hline 9 & 8,95 & 8,7 & 0,83 \\
\hline 10 & 12,65 & 11,9 & 0,83 \\
\hline 11 & 15,65 & 14,8 & 0,83 \\
\hline 12 & 16,8 & 15,7 & 0,83 \\
\hline 13 & 17,4 & 16,6 & 0,83 \\
\hline 14 & 17,8 & 16,4 & 0,83 \\
\hline 15 & 18,1 & 14,4 & 0,83 \\
\hline 16 & 15,9 & 15,0 & 0,83 \\
\hline 17 & 1,1 & 1,1 & 0 \\
\hline$s_{1}$ & \multicolumn{2}{|c|}{ Loststellung } & \\
\hline 18 & 1,3 & 1,3 & 0 \\
\hline 19 & 17,8 & 17,05 & 0 \\
\hline 20 & 17,05 & 16,55 & 0,83 \\
\hline 21 & 20,5 & 19,7 & 0,83 \\
\hline 22 & 1,3 & 1,3 & 0 \\
\hline 23 & 20,5 & 19,3 & 0 \\
\hline 24 & 19,3 & 19,3 & 0,83 \\
\hline 25 & 19,3 & 19,3 & 0,94 \\
\hline 26 & 23,1 & 22,2 & 0,94 \\
\hline 27 & 27,1 & 25,5 & 0,94 \\
\hline 28 & 31,1 & 29,45 & 0,94 \\
\hline 29 & 36,1 & 33,3 & 0,94 \\
\hline 30 & 37,1 & 34,1 & 0,94 \\
\hline
\end{tabular}

\begin{tabular}{|c|c|c|c|}
\hline $\mathrm{S}_{2}$ & \multicolumn{2}{|c|}{ Laststellung } & \\
\hline $\begin{array}{l}\text { Lost - } \\
\text { slufe }\end{array}$ & $\begin{array}{c}Q_{A} \\
t \\
\end{array}$ & $\begin{array}{c}Q_{E} \\
t\end{array}$ & $\begin{array}{c}\mathrm{m}_{\mathrm{q}} \\
\mathrm{mt} / \mathrm{m}^{\prime}\end{array}$ \\
\hline 1 & 1,1 & 1,1 & 0 \\
\hline 2 & 6,1 & 5,7 & 0 \\
\hline 3 & 8,6 & 8,1 & 0 \\
\hline 4 & 8,1 & 7,9 & 0,55 \\
\hline 5 & 1,1 & 1,1 & 0 \\
\hline 6 & 6,1 & 6,0 & 0 \\
\hline 7 & 6,0 & 6,0 & 0,55 \\
\hline 8 & 8,6 & 8,2 & 0,55 \\
\hline 9 & 1,1 & 1,1 & 0 \\
\hline 10 & 8,6 & 8,3 & 0,55 \\
\hline 11 & 8,3 & 8,0 & 0,88 \\
\hline 12 & 2,1 & 9,5 & 0,88 \\
\hline 13 & 12,6 & 12,1 & 0,88 \\
\hline 14 & 14,6 & 13,9 & 0,88 \\
\hline 15 & 16,1 & 15,4 & 0,88 \\
\hline 16 & 15,3 & 15,1 & 0,88 \\
\hline 17 & 16,9 & 16,1 & 0,88 \\
\hline 18 & 17,4 & 16,5 & 0,88 \\
\hline 19 & 17,9 & 16,8 & 0,88 \\
\hline 20 & 18,3 & 13,8 & 0,88 \\
\hline 29 & 15,3 & 14,3 & 0,88 \\
\hline \multicolumn{4}{|c|}{ Loststellung } \\
\hline 22 & 1,1 & 1,1 & 0 \\
\hline 23 & 8,6 & 8,1 & 0 \\
\hline 24 & 8,1 & 7,95 & 0,88 \\
\hline 25 & 15,3 & 14,5 & 0,88 \\
\hline 26 & 15,8 & 15,35 & 1,52 \\
\hline 27 & 1,1 & 1,1 & 0 \\
\hline 28 & 14,5 & 14,5 & 1,52 \\
\hline 29 & 14,5 & 14,5 & 1,74 \\
\hline 30 & 21,1 & 20,15 & 1,74 \\
\hline 31 & 26,1 & 24,95 & 1,74 \\
\hline 32 & 31,1 & 28,4 & 1,74 \\
\hline 33 & 31,6 & 29,6 & 1,74 \\
\hline
\end{tabular}

Tabelle 5: Belastungsablauf Balken $S_{1}$ und $S_{2}$ 


\begin{tabular}{|c|c|c|c|}
\hline $\mathrm{S}_{3}$ & \multicolumn{2}{|c|}{ Loststellung } & \\
\hline $\begin{array}{l}\text { Last - } \\
\text { stufe }\end{array}$ & $\begin{array}{c}Q_{A} \\
t\end{array}$ & $\begin{array}{c}Q_{E} \\
t\end{array}$ & $\mathrm{~m}_{\mathrm{mt}} / \mathrm{m}^{\prime}$ \\
\hline 1 & 1,1 & 1,1 & 0 \\
\hline 2 & 7,25 & 6,9 & 0 \\
\hline 3 & 6,9 & 6,8 & 0,4 \\
\hline 4 & 6,8 & 6,7 & 0,54 \\
\hline 5 & 1,3 & 1,3 & 0 \\
\hline 6 & 7,25 & 7,0 & 0 \\
\hline 7 & 13,4 & 12,6 & 0 \\
\hline 8 & 12,6 & 12,4 & 0,4 \\
\hline 9 & 12,4 & 12,3 & 0,54 \\
\hline 10 & 1,3 & 1,3 & 0 \\
\hline 11 & 12,6 & 12,2 & 0 \\
\hline 12 & 12,2 & 12,0 & 0,54 \\
\hline 13 & 12,0 & 11,7 & 0,94 \\
\hline 14 & 16,1 & 15,5 & 0,94 \\
\hline 15 & 19,1 & 18,0 & 0,94 \\
\hline 16 & 22,1 & 21,1 & 0,94 \\
\hline 17 & 22,8 & 22,3 & 0,94 \\
\hline 18 & 25,1 & 23,7 & 0,94 \\
\hline 19 & 26,2 & 24,1 & 0,94 \\
\hline 20 & 26,3 & 24,3 & 0,94 \\
\hline 21 & 26,3 & 24,7 & 0,94 \\
\hline 22 & 26,6 & 22,5 & 0,94 \\
\hline \multicolumn{4}{|c|}{ Loststellung } \\
\hline 23 & 1,1 & 1,1 & 0 \\
\hline 24 & 13,4 & 12,6 & 0 \\
\hline 25 & 12,9 & 12,6 & 0,54 \\
\hline 26 & 20,5 & 19,8 & 0,54 \\
\hline 27 & 19,8 & 19,3 & 0,82 \\
\hline 28 & 1,2 & 1,2 & 0 \\
\hline 29 & 19,85 & 19,7 & 0,82 \\
\hline 30 & 19,7 & 19,45 & 0,94 \\
\hline 31 & 19,45 & 18,85 & 1,43 \\
\hline 32 & 24,1 & 23,2 & 1,43 \\
\hline 33 & 29,1 & 28,05 & 1,43 \\
\hline 34 & 33,1 & 30,95 & 1,43 \\
\hline 35 & 36,1 & 33,9 & 1,43 \\
\hline 36 & 38,4 & 33,7 & 1,43 \\
\hline
\end{tabular}

Tabelle 6 : Belastungsablauf Balken $\mathrm{S}_{3}$ und $\mathrm{S}_{4}$

\begin{tabular}{|c|c|c|c|}
\hline \multirow{2}{*}{\begin{tabular}{l}
\multicolumn{1}{c}{$\mathrm{S}_{4}$} \\
Lost - \\
stufe
\end{tabular}} & \multicolumn{2}{|c|}{ Loststellung } & \\
\hline & $\stackrel{Q_{A}}{t}$ & $\begin{array}{c}Q_{E} \\
\dagger\end{array}$ & $\mathrm{m}_{\mathrm{q}} \mathrm{m}^{\prime}$ \\
\hline 1 & 1,1 & 1,1 & 0 \\
\hline 2 & 5,55 & 5,25 & 0 \\
\hline 3 & 5,25 & 5,25 & 0,41 \\
\hline 4 & 5,25 & 5,05 & 0,62 \\
\hline 5 & 5,05 & 5,0 & 0,82 \\
\hline 6 & 1,1 & 1,1 & 0 \\
\hline 7 & 5,85 & 5,65 & 0 \\
\hline 8 & 8,25 & 7,8 & 0 \\
\hline 9 & 7,8 & 7,75 & 0,41 \\
\hline 10 & 7,75 & 7,65 & 0,62 \\
\hline 11 & 7,65 & 7,6 & 0,72 \\
\hline 12 & 1,1 & 1,1 & 0 \\
\hline 13 & 8,25 & 7,9 & 0 \\
\hline 14 & 10,6 & 10,05 & 0 \\
\hline 15 & 10,05 & 10,05 & 0,41 \\
\hline 16 & 10,05 & 9,9 & 0,62 \\
\hline 17 & 8,25 & 8,25 & 0,62 \\
\hline 18 & 10,6 & 10,2 & 0,62 \\
\hline 19 & 10,2 & 10,2 & 0,82 \\
\hline 20 & 1,1 & 1,1 & 0 \\
\hline 21 & 10,6 & 10,15 & 0,82 \\
\hline 22 & 10,15 & 9,7 & 1,42 \\
\hline 23 & 12,7 & 12,6 & 1,42 \\
\hline 24 & 15,6 & 14,6 & 1,42 \\
\hline 25 & 18,0 & 17,0 & 1,42 \\
\hline 26 & 19,85 & 18,4 & 1,42 \\
\hline 27 & 21,1 & 19,2 & 1,42 \\
\hline 28 & 20,8 & 20,0 & 1,42 \\
\hline 29 & 23,5 & 21,3 & 1,42 \\
\hline 30 & 23,0 & 21,9 & 1,42 \\
\hline \multicolumn{4}{|c|}{ Loststellung } \\
\hline 31 & 1,1 & 1,1 & 0 \\
\hline 32 & 10,6 & 10,1 & 0 \\
\hline 33 & 17,1 & 16,1 & 0 \\
\hline 34 & 1,1 & 1,1 & 0 \\
\hline 35 & 17,1 & 16,9 & 0 \\
\hline 36 & 16,9 & 16,9 & 0,82 \\
\hline 37 & 16,9 & 16,85 & 1,09 \\
\hline 38 & 21,1 & 20,2 & 1,09 \\
\hline 39 & 25,1 & 24,0 & 1,09 \\
\hline 40 & 29,1 & 27,6 & 1,09 \\
\hline 41 & 32,1 & 30,1 & 1,09 \\
\hline 42 & 34,1 & 30,35 & 1,09 \\
\hline 43 & 33,85 & 29,1 & 1,09 \\
\hline
\end{tabular}




\begin{tabular}{|c|c|c|c|}
\hline$S_{5}$ & \multicolumn{2}{|c|}{ Laststellung } & \\
\hline $\begin{array}{l}\text { Lost- } \\
\text { stufe }\end{array}$ & $\begin{array}{c}Q_{A} \\
t\end{array}$ & $Q_{E}$ & $\begin{array}{c}\mathrm{m}_{\mathrm{q}} \\
\mathrm{mt} / \mathrm{m}^{\prime}\end{array}$ \\
\hline 1 & 1,1 & 1,1 & 0 \\
\hline 2 & 6,7 & 6,2 & 0 \\
\hline 3 & 9,5 & 9,0 & 0 \\
\hline 4 & 1,1 & 1,1 & 0 \\
\hline 5 & 9,5 & 9,1 & 0 \\
\hline 6 & 9,1 & 9,0 & 0,51 \\
\hline 7 & 9,0 & 8,6 & 0,765 \\
\hline 8 & 8,6 & 8,5 & 1,02 \\
\hline 9 & 1,1 & 1,1 & 0 \\
\hline 10 & 9,5 & 9,3 & 0 \\
\hline 11 & 12,3 & 11,8 & 0 \\
\hline 12 & 11,8 & 11,8 & 0,51 \\
\hline 13 & 11,8 & 11,7 & 0,765 \\
\hline 14 & 11,7 & 11,6 & 1,02 \\
\hline 15 & 1,1 & 1,1 & 0 \\
\hline 16 & 12,3 & 11,9 & 0 \\
\hline 17 & 11,9 & 11,7 & 1,02 \\
\hline 18 & 11,7 & 11,5 & 1,77 \\
\hline 19 & 16,1 & 15,3 & 1,77 \\
\hline 20 & 19,1 & 18,1 & 1,77 \\
\hline 21 & 22,1 & 20,6 & 1,77 \\
\hline 22 & 23,3 & 21,9 & 1,77 \\
\hline 23 & 24,1 & 22,7 & 1,77 \\
\hline 24 & 25,2 & 22,6 & 1,77 \\
\hline 25 & 25,4 & 22,7 & 1,77 \\
\hline 26 & 26,1 & 24,6 & 1,77 \\
\hline $\mathrm{S}_{\mathrm{s}}$ & Losts & llung & \\
\hline 27 & 1,1 & 1,1 & 0 \\
\hline 28 & 19,8 & 18,35 & 0 \\
\hline 29 & 18,35 & 17,75 & 1,02 \\
\hline 30 & 1,1 & 1,1 & 0 \\
\hline 31 & 19,8 & 19,2 & 0 \\
\hline 32 & 19,2 & 19,0 & 1,02 \\
\hline 33 & 19,0 & 19,0 & 1,35 \\
\hline 34 & 24,1 & 23,2 & 1,35 \\
\hline 35 & 28,1 & 26,75 & 1,35 \\
\hline 36 & 32,1 & 30,3 & 1,35 \\
\hline 37 & 35,1 & 32,25 & 1,35 \\
\hline 38 & 36,5 & 32,0 & 1,35 \\
\hline
\end{tabular}

Tabelle 7: Belastungsablauf $\mathrm{S}_{5}$ und $\mathrm{S}_{6}$

\begin{tabular}{|c|c|c|c|}
\hline $\mathrm{S}_{6}$ & \multicolumn{2}{|c|}{ Laststellung } & \\
\hline $\begin{array}{l}\text { Last- } \\
\text { stufe }\end{array}$ & $\begin{array}{c}Q_{A} \\
t\end{array}$ & $\begin{array}{c}Q_{E} \\
t \\
\end{array}$ & $\begin{array}{c}\mathrm{m}_{\mathrm{q}} \\
\mathrm{mt} / \mathrm{m}^{\prime}\end{array}$ \\
\hline 1 & 1,1 & 1,1. & 0 \\
\hline 2 & 6,7 & 6,0 & 0 \\
\hline 3 & 8,55 & 8,05 & 0 \\
\hline 4 & 1,1 & 1,1 & 0 \\
\hline 5 & 6,7 & 6,5 & 0 \\
\hline 6 & 6,5 & 6,5 & 0,405 \\
\hline 7 & 6,5 & 6,25 & 0,54 \\
\hline 8 & 1,1 & 1,1 & 0 \\
\hline 9 & 6,7 & 6,55 & 0 \\
\hline 10 & 8,55 & 8,25 & 0 \\
\hline 11 & 8,25 & 7,95 & 0,405 \\
\hline 12 & 7,95 & 7,75 & 0,54 \\
\hline 13 & 10,1 & 9,4 & 0,54 \\
\hline 14 & 11,6 & 11,1 & 0,54 \\
\hline 15 & 13,15 & 12,5 & 0,54 \\
\hline 16 & 14,65 & 13,95 & 0,54 \\
\hline 17 & 15,6 & 14,8 & 0,54 \\
\hline 18 & 16,6 & 15,45 & 0,54 \\
\hline 19 & 17,25 & 16,15 & 0,54 \\
\hline 20 & 18,05 & 16,25 & 0,54 \\
\hline 21 & 18,1 & 14,5 & 0,54 \\
\hline \multicolumn{4}{|c|}{ Laststellung } \\
\hline 22 & 1,1 & 1,1 & 0 \\
\hline 23 & 7,25 & 7,15 & 0 \\
\hline 24 & 13,45 & 13,05 & 0 \\
\hline 25 & 13,05 & 13,05 & 0,405 \\
\hline 26 & 13,05 & 12,9 & 0,54 \\
\hline 27 & 1,1 & 1,1 & 0 \\
\hline 28 & 13,45 & 13,25 & 0 \\
\hline 29 & 13,25 & 13,1 & 0,54 \\
\hline 30 & 13,1 & 13,1 & 0,69 \\
\hline 31 & 13,45 & 13,45 & 0,74 \\
\hline 32 & 13,45 & 13,45 & 0,91 \\
\hline 33 & 13,45 & 13,35 & 0,95 \\
\hline 34 & 13,35 & 13,3 & 1,03 \\
\hline 35 & 13,3 & 13,3 & 1,1 \\
\hline 36 & 13,3 & 13,3 & 1,24 \\
\hline 37 & 13,3 & 13,15 & 1,38 \\
\hline 38 & 13,15 & 13,15 & 1,46 \\
\hline 39 & 13,15 & 13,15 & 1,55 \\
\hline 40 & 13,15 & 12,85 & 1,58 \\
\hline 41 & 12,85 & 12,7 & 1,58 \\
\hline
\end{tabular}




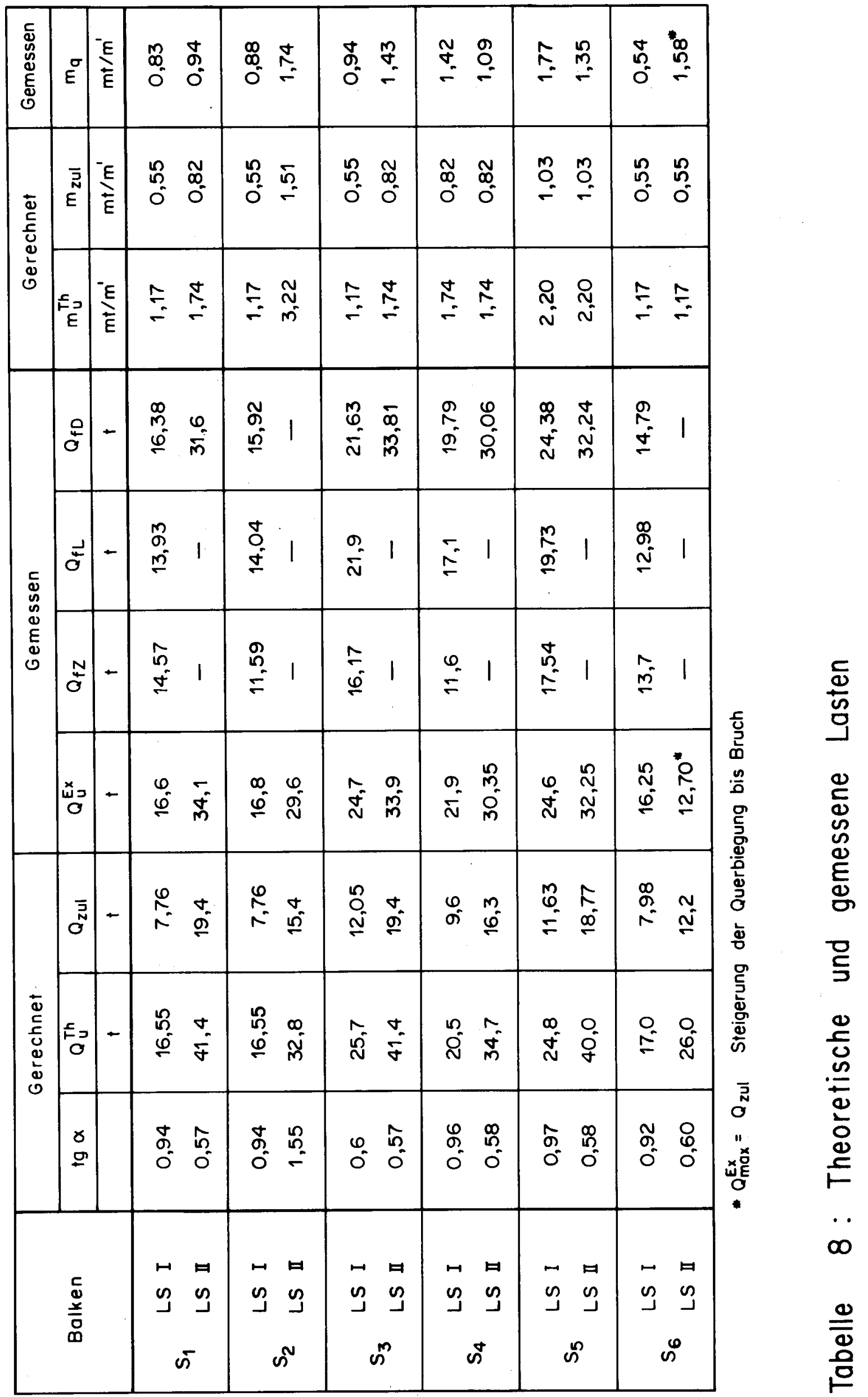



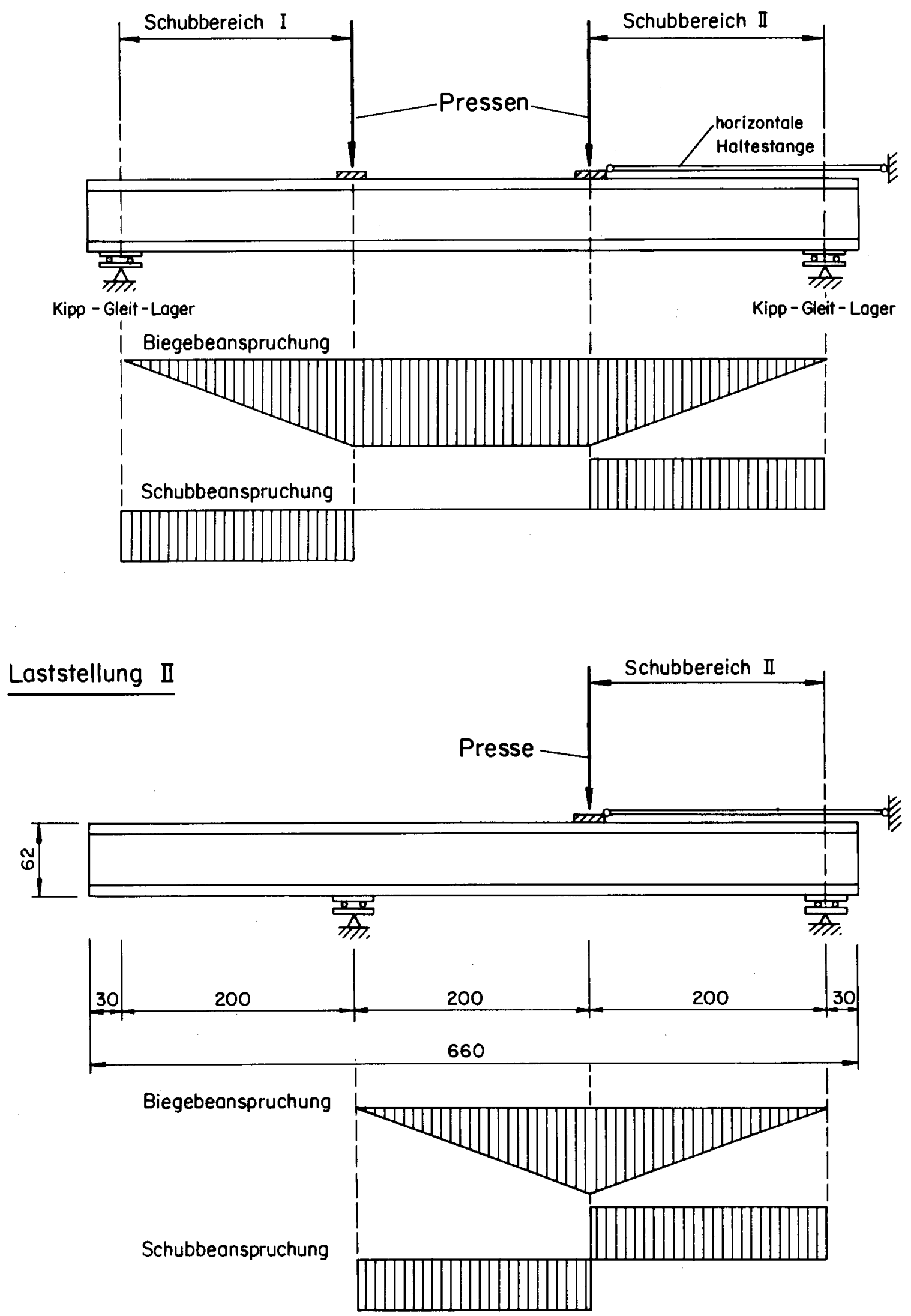

Bild 1 : Belastung in Längsrichtung (Masse in $\mathrm{cm}$ ) 


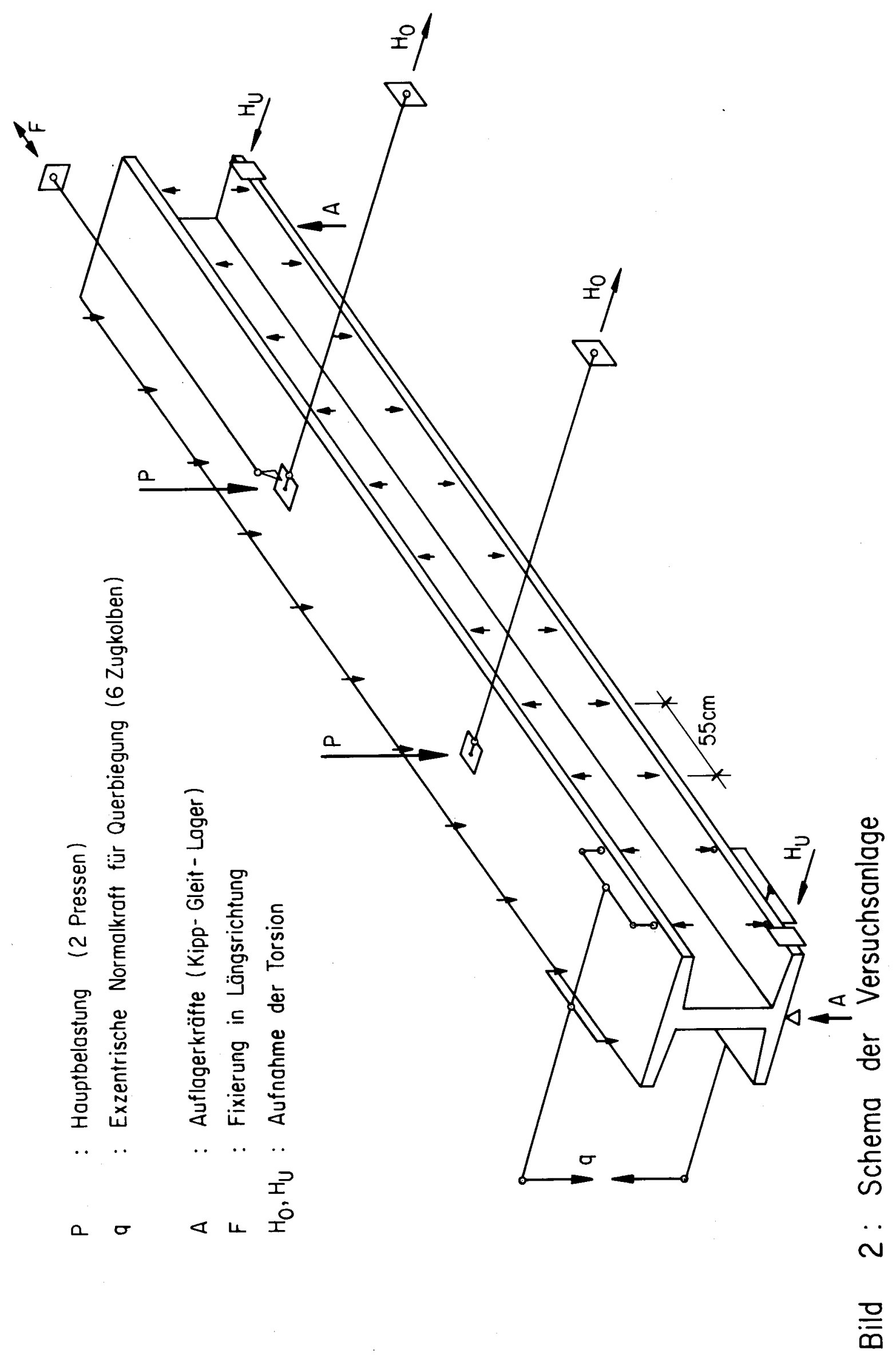




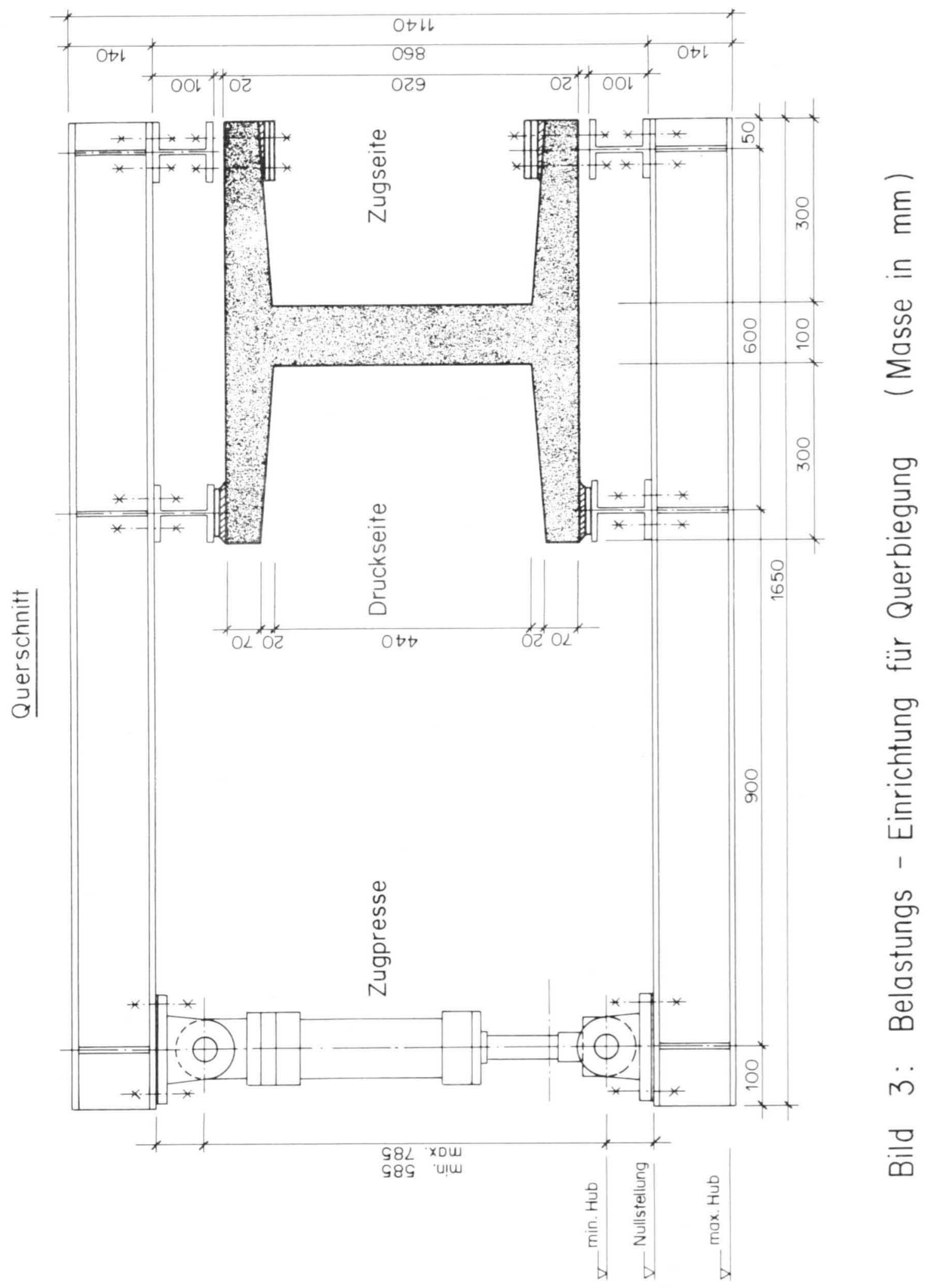




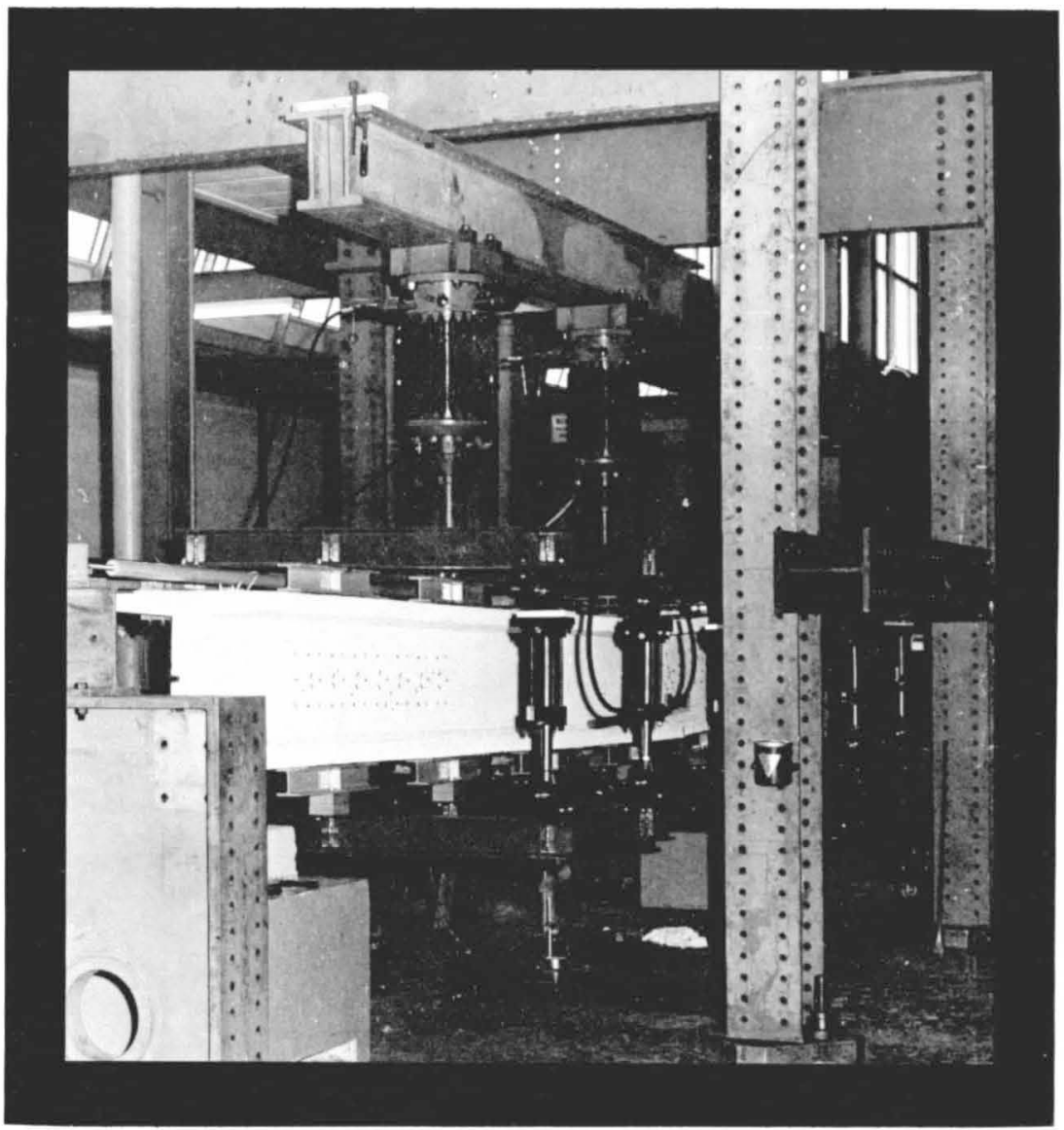

Bild 4: Gesamtansicht der Versuchsanlage

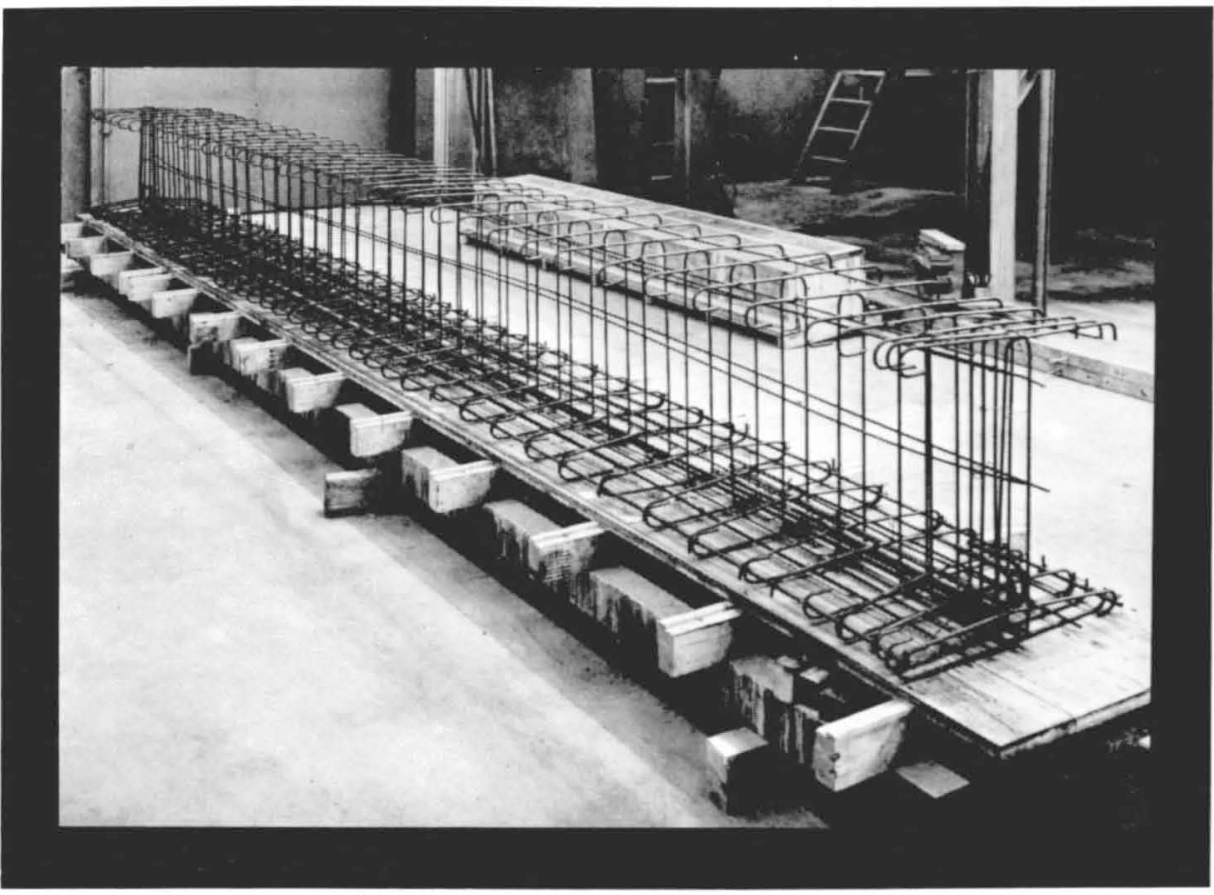

Bild 5: Bewehrungskorb 


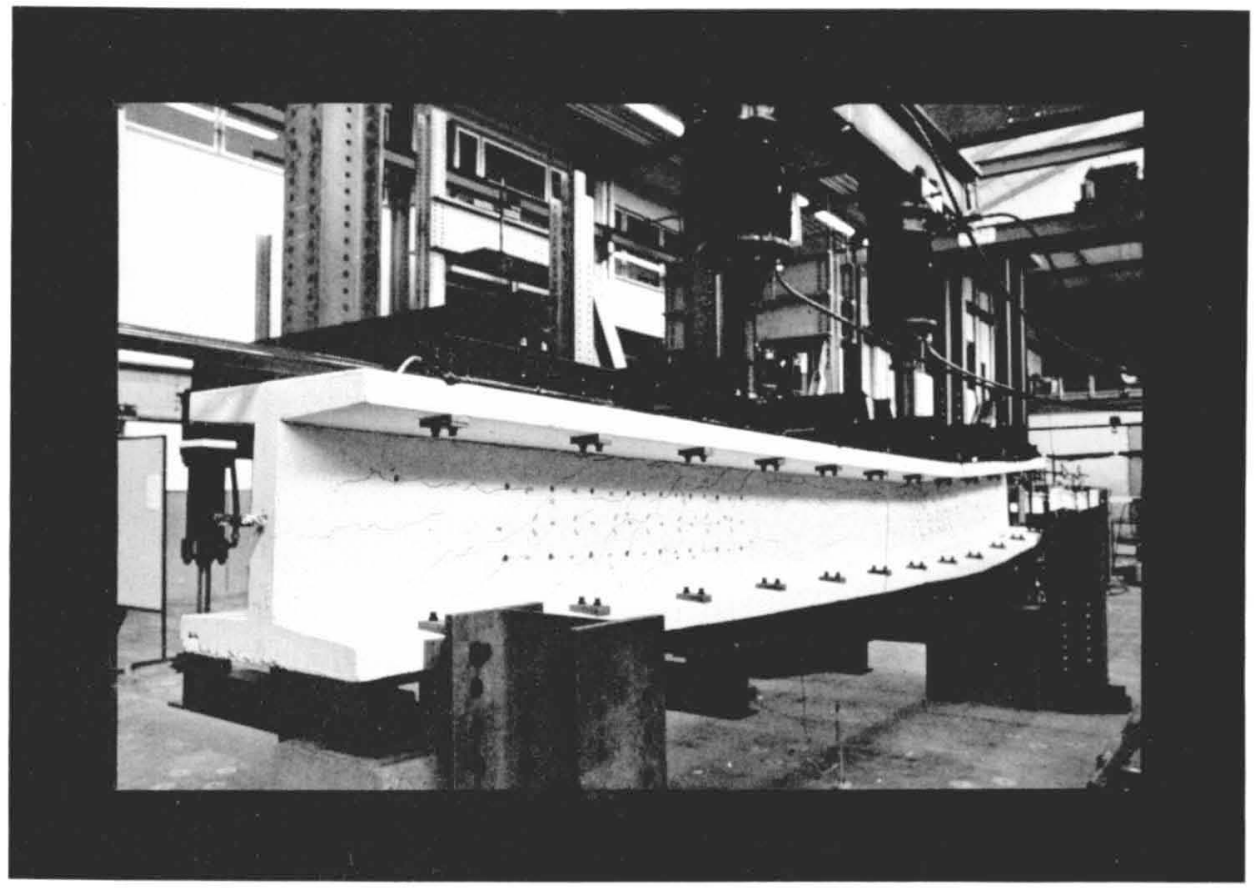

Bild 6: Ansicht Vorderseite des Versuchsbalkens

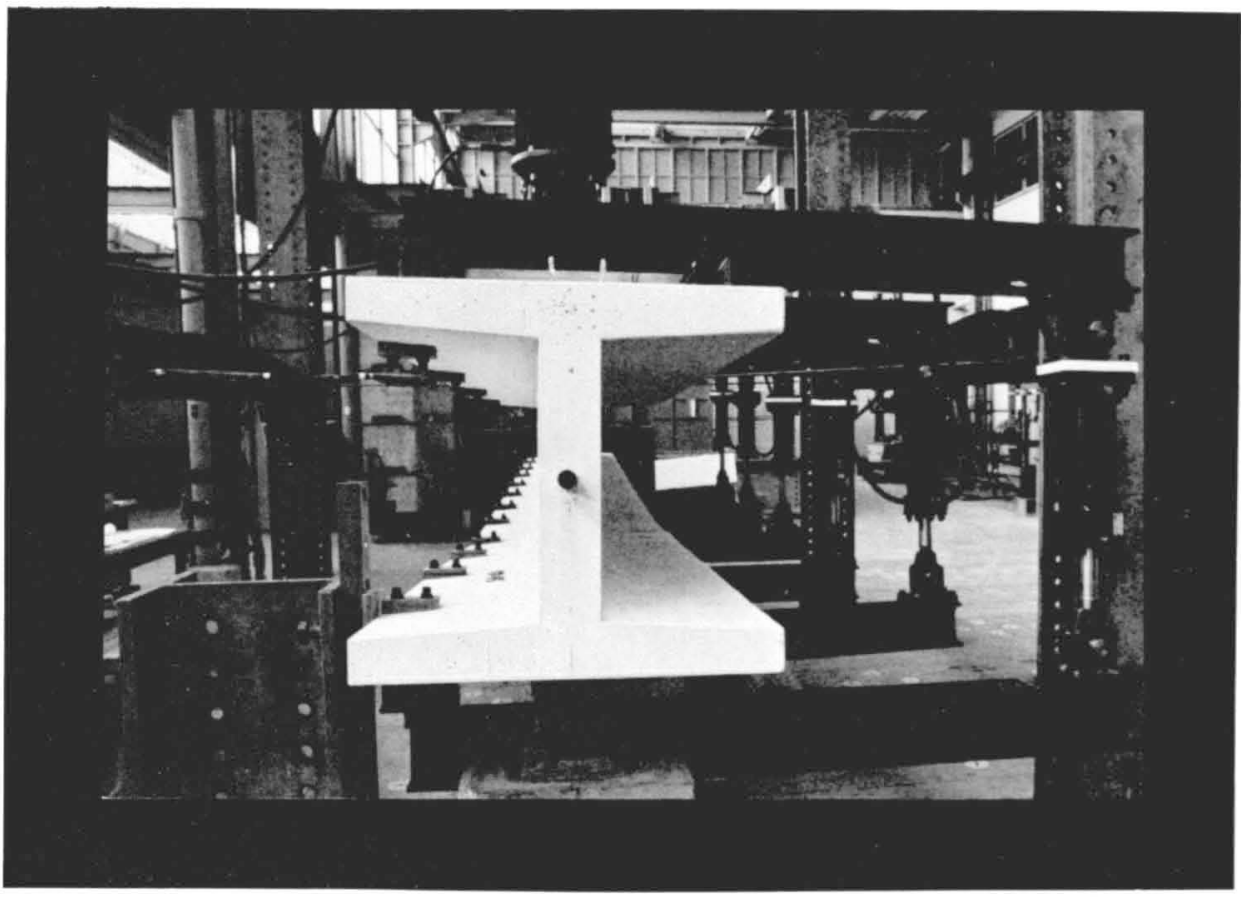

Bild 7: Querschnitt, Belastungseinrichtung für Querbiegung 

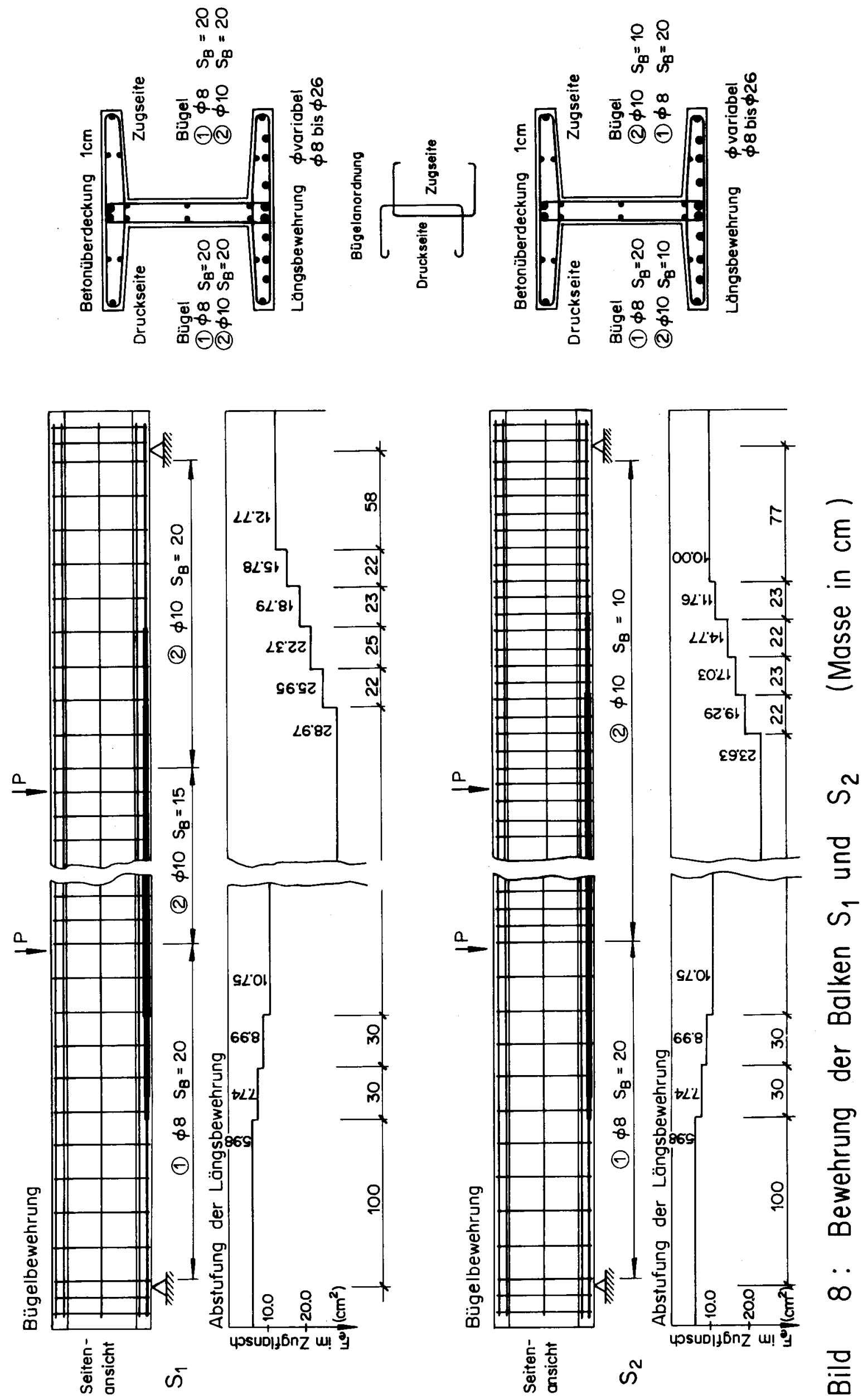

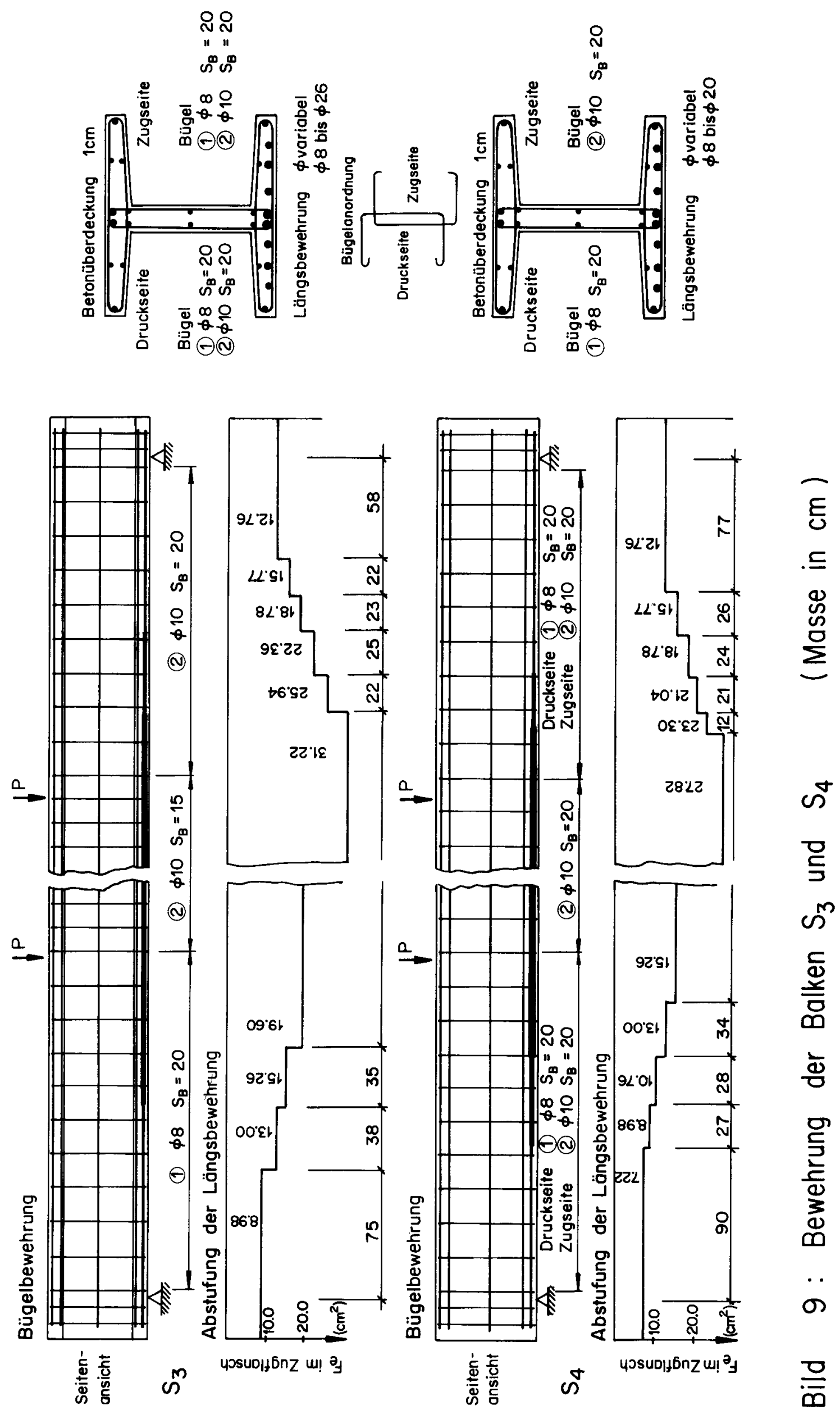

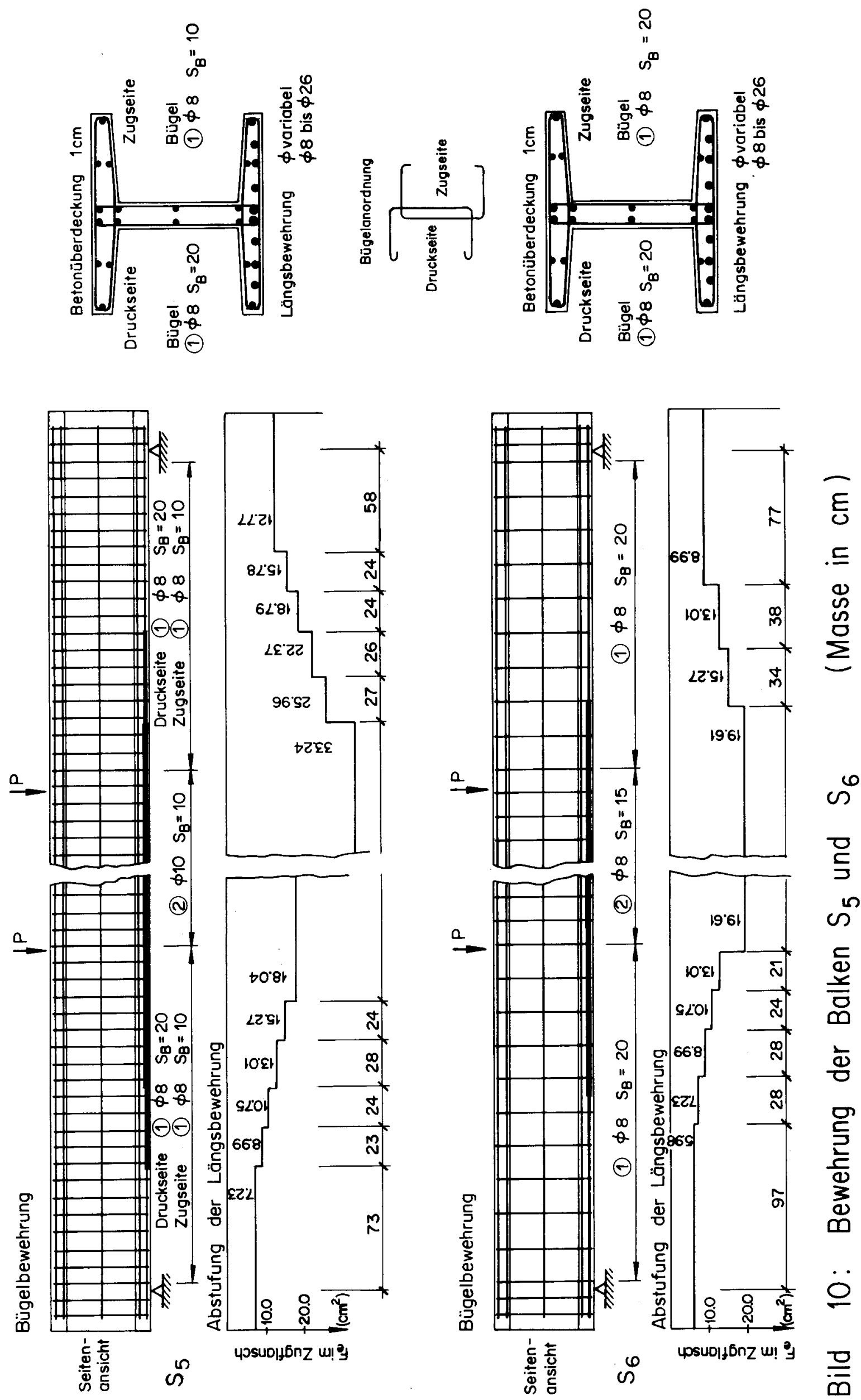


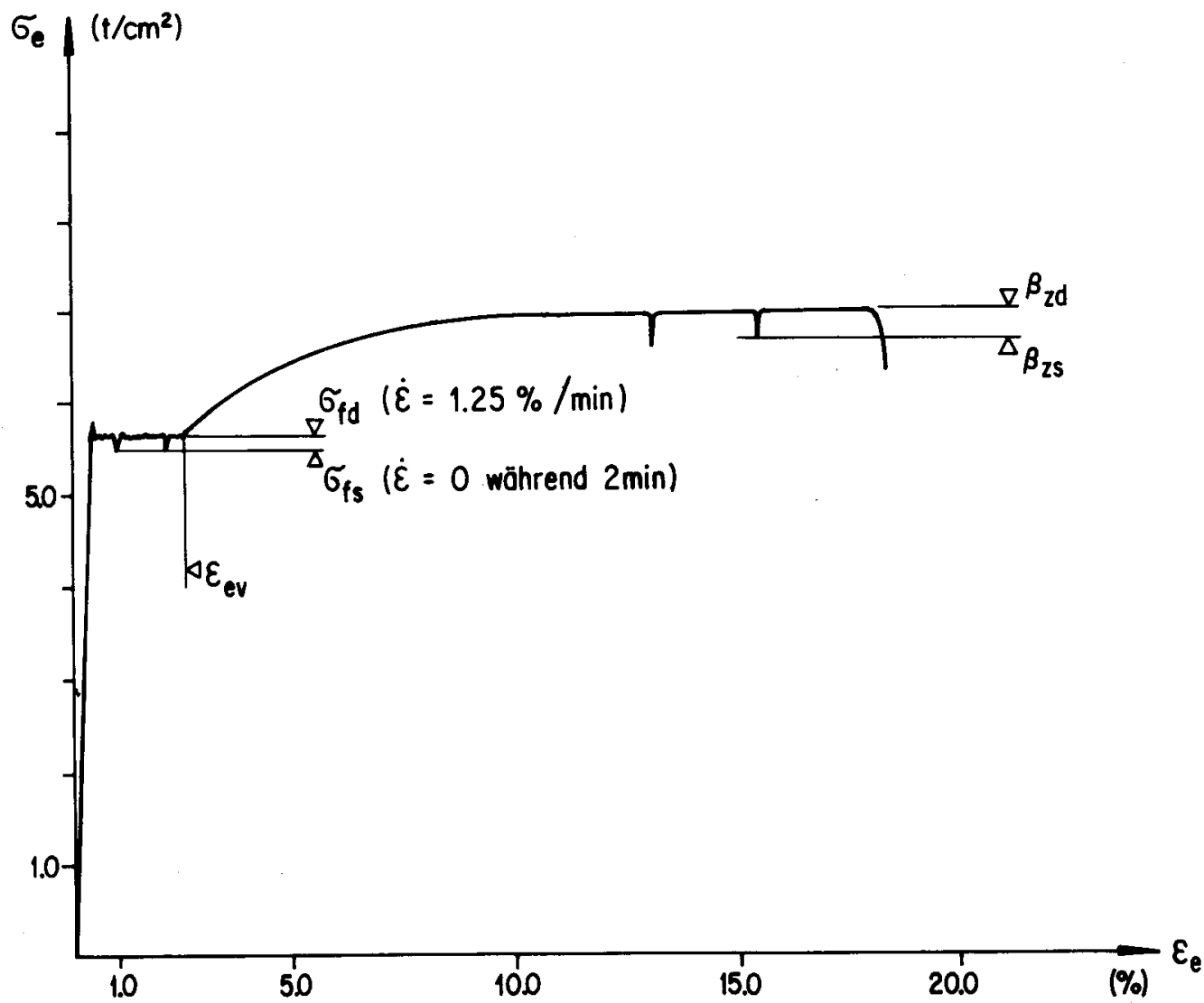

Bild 11: Spannungs - Dehnungs - Diagramm eines BOX-Stahles $\phi 8 \mathrm{~mm}$

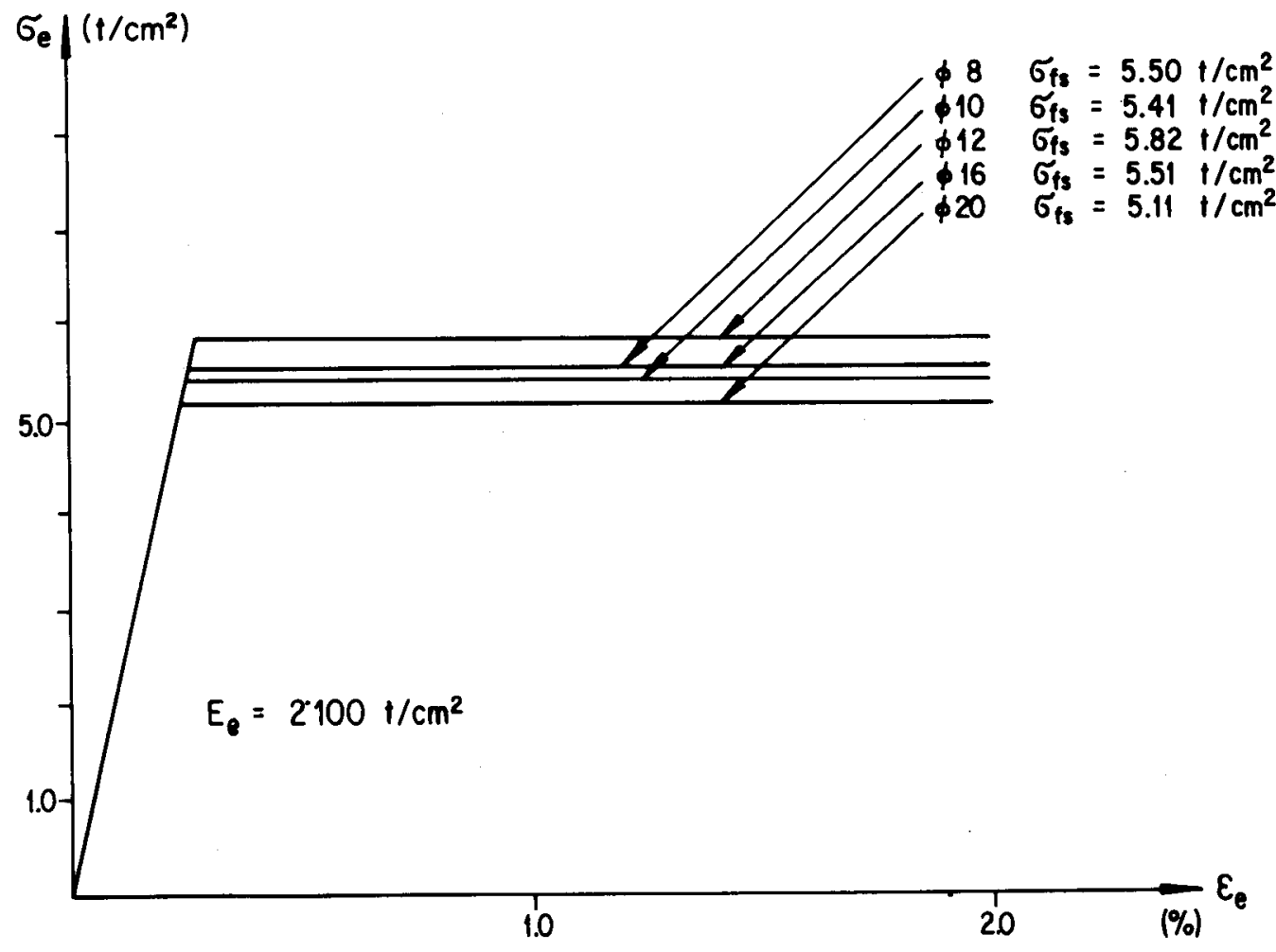

Bild 12: Gemittelte Spannungs-Dehnungs-Diagramme BOX-Stahl 


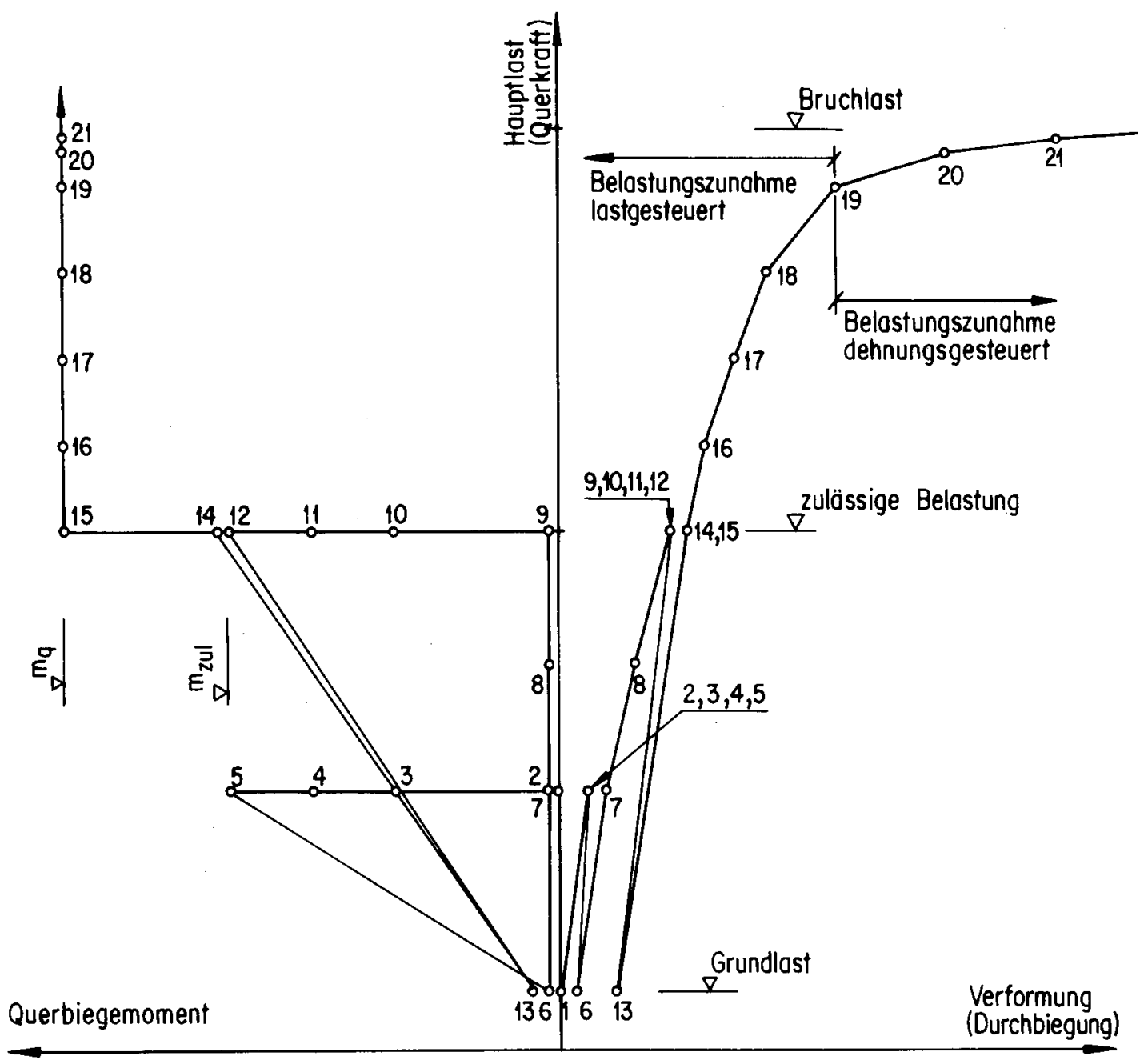

Bild 13: Generelles Belastungsschema

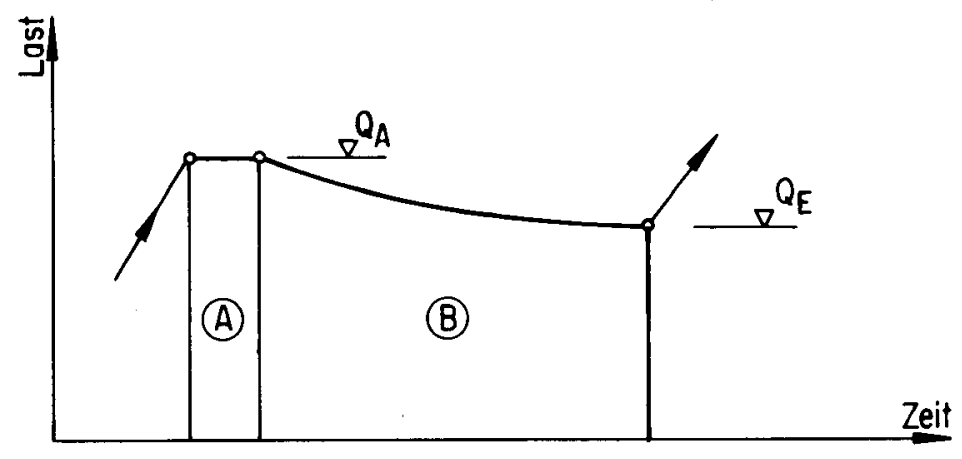

(A) : Kroft konstant während 2 Min

(B): Durchbiegung konstont (Messung der Verformungen)

Bild 14: Schema einer Laststufe 
Ansicht Zugseite
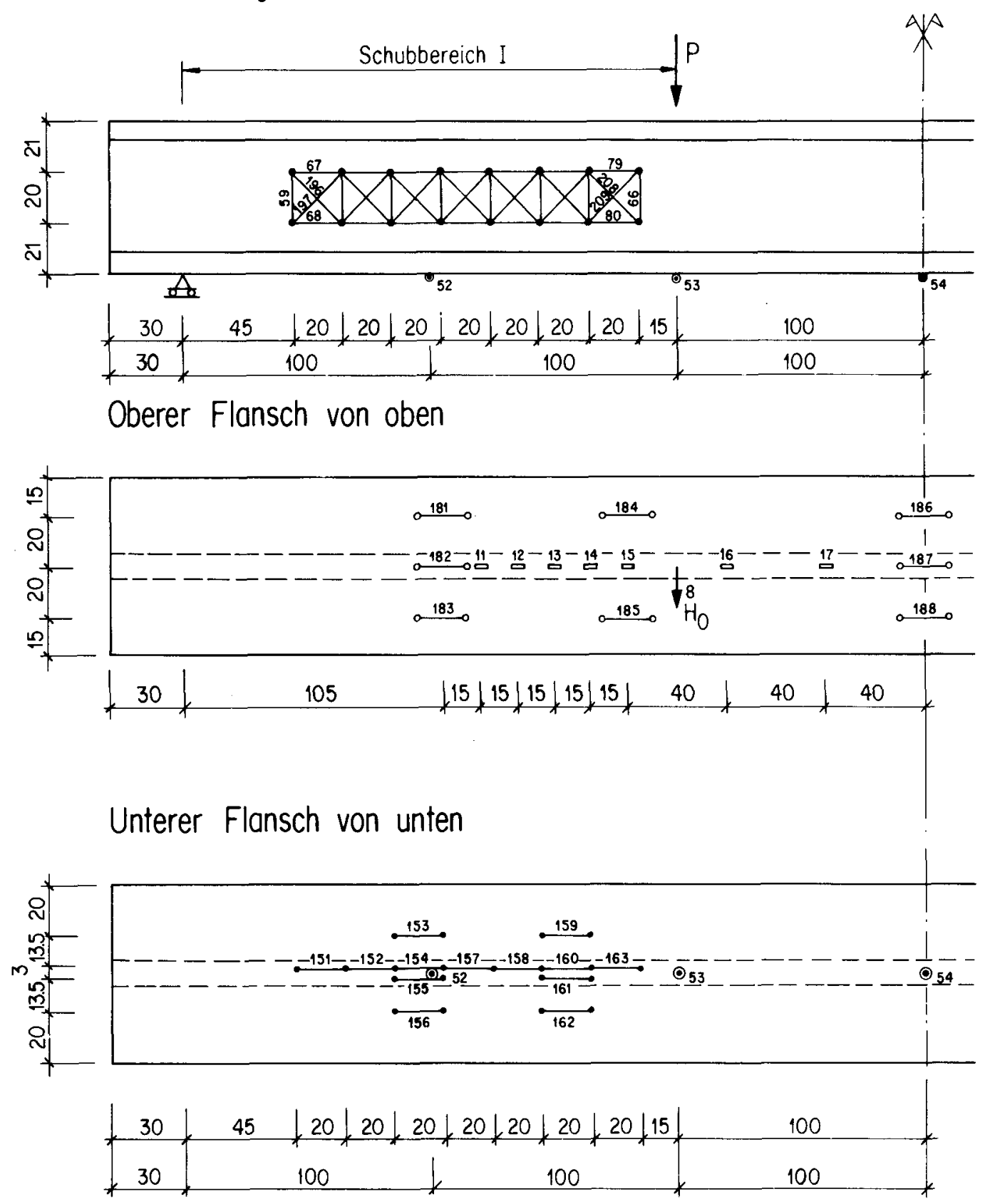

\begin{tabular}{|c|c|c|c|c|c|c|c|}
\hline $\begin{array}{c}\text { Messstelle } \\
\mathrm{Nr} .\end{array}$ & $\begin{array}{r}\text { Schub- } \\
\text { bereich }\end{array}$ & Messung & Messgerät & $\begin{array}{c}\text { Messstelle } \\
\text { Nr. }\end{array}$ & $\begin{array}{l}\text { Schub- } \\
\text { bereich }\end{array}$ & Messung & Messgerät \\
\hline $1-6$ & $I+I I$ & Kontrolle Querbiegemoment & & $105-112$ & 1 & Bügeldehnung Druckseite & \\
\hline $8-9$ & I + II & Kroftmessung $\mathrm{H}_{\mathrm{O}}$ & $\begin{array}{l}\text { Dehnungs- } \\
\text { mess- }\end{array}$ & $113-126$ & I & Horizontaler Bügelabstand & \\
\hline $11-17$ & I & Betonstouchung & streifen & $128-135$ & I & Bügeldehnung Druckseite & $20 \mathrm{~cm}$ \\
\hline $18-24$ & II & Betonstouchung & & $136-149$ & II & Horizontoler Bügelabstand & Defor - \\
\hline $52-56$ & $\mathrm{I}+\mathrm{I}$ & Durchbiegung & Messstab & $151-163$ & I & Dehnung Löngsbewehrung & meter \\
\hline $59-66$ & I & Bügeldehnung Zugseite & & $164-179$ & II & Dehnung Löngsbewehrung & \\
\hline $67-80$ & I & Horizontaler Bügelabstand & $20 \mathrm{~cm}$ & $181-193$ & $I+\mathbb{I}$ & Betonstauchung & \\
\hline $82-89$ & II & Bügeldehnung Zugseite & Deformeter & $196-223$ & $I+I$ & Diagonalen Zugseite & $28 \mathrm{~cm}$ \\
\hline $90-103$ & 【 & Horizontaler Bügelabstand & & $225-252$ & $I+\mathbb{I}$ & Diagonalen Druckseite & $\begin{array}{l}\text { Detor- } \\
\text { meter }\end{array}$ \\
\hline
\end{tabular}

\begin{tabular}{lll}
$\longrightarrow$ Stohl - Messstelle & $\odot$ Durchbiegungs - Messstelle \\
\hdashline$\quad$ Beton-Messstelle & $\square \quad$ Dehnungsmessstreifen
\end{tabular}

Bild 15: Messstellenplan (Schubbereich II analog)(Masse in $\mathrm{cm}$ ) 


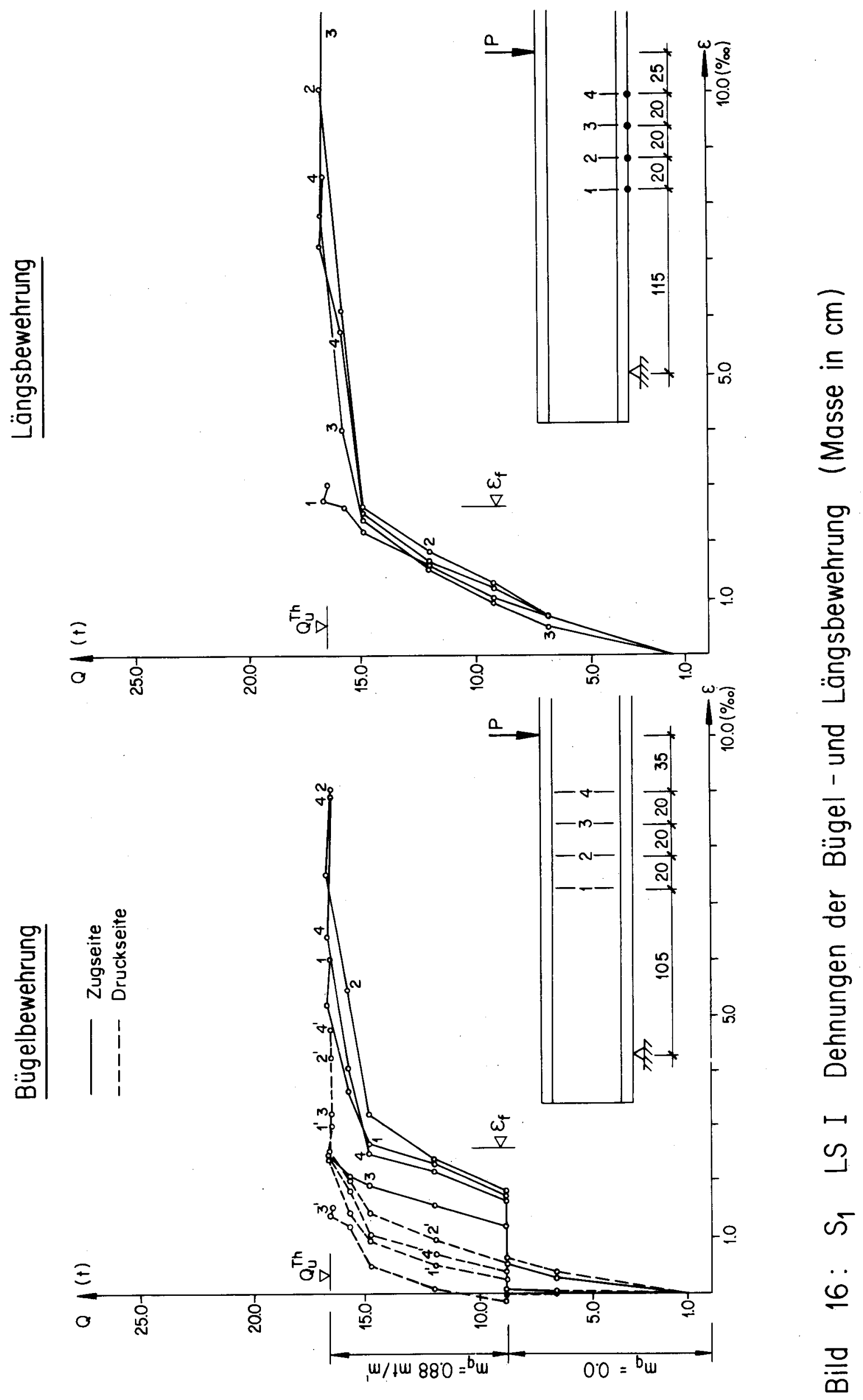




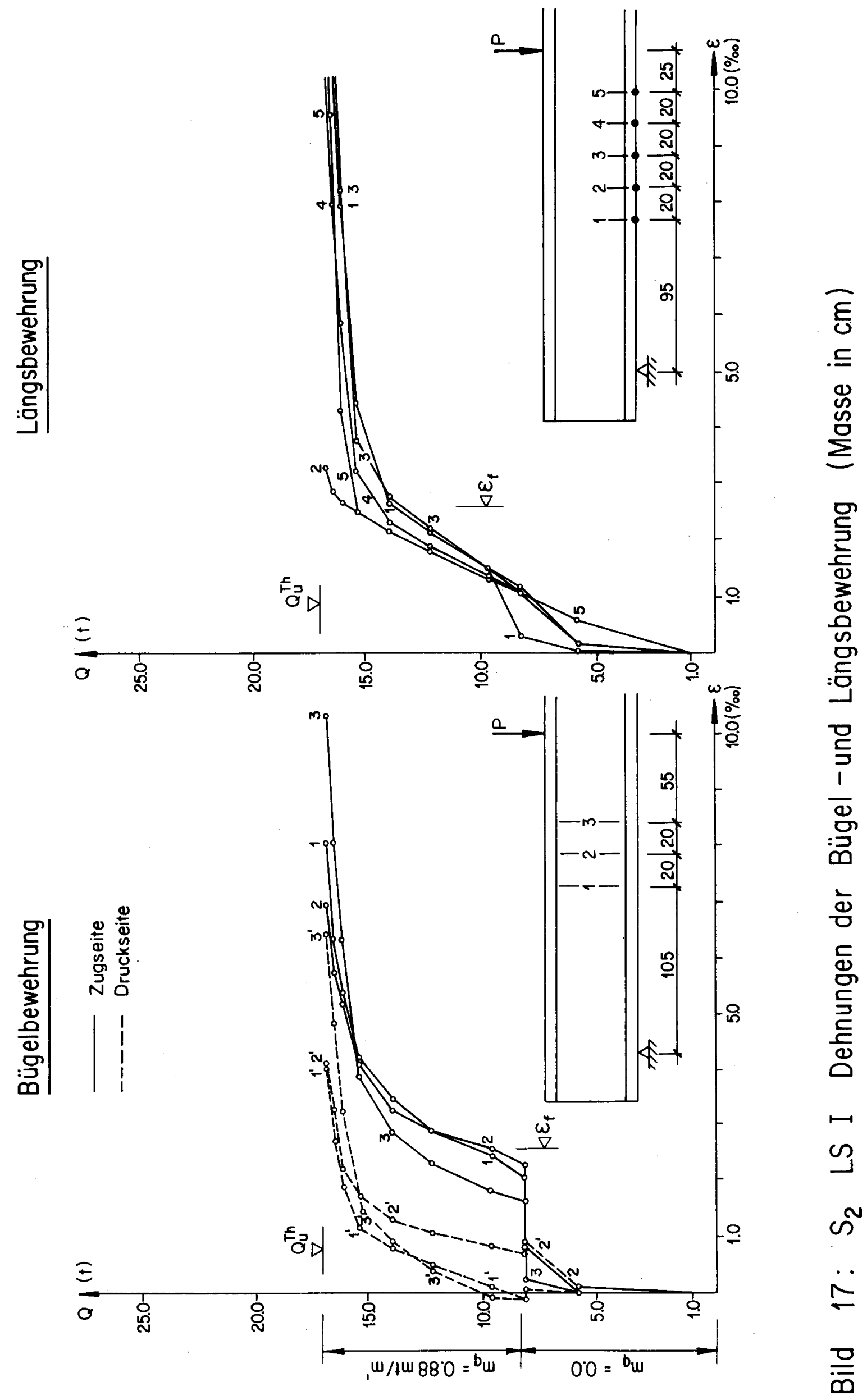




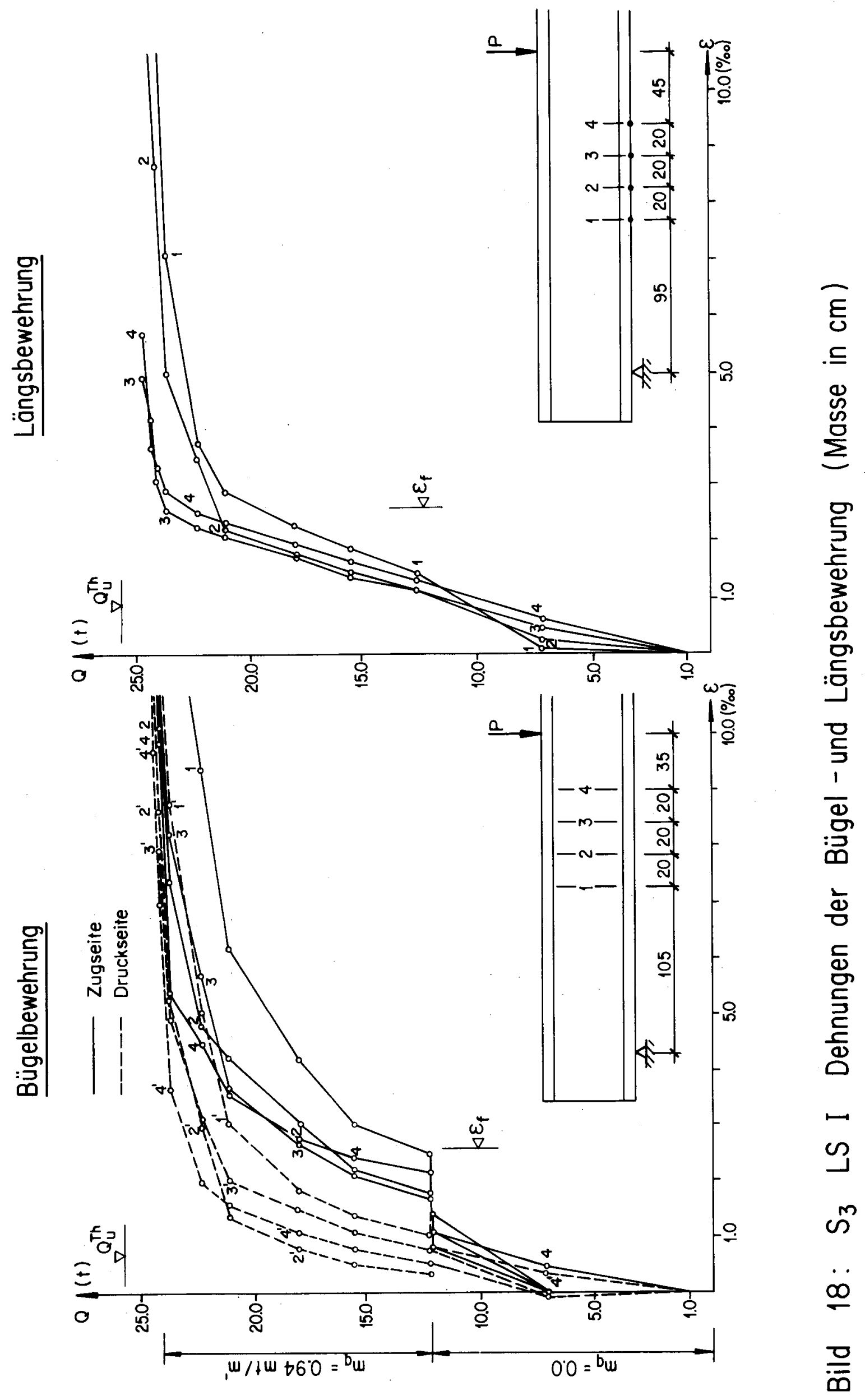




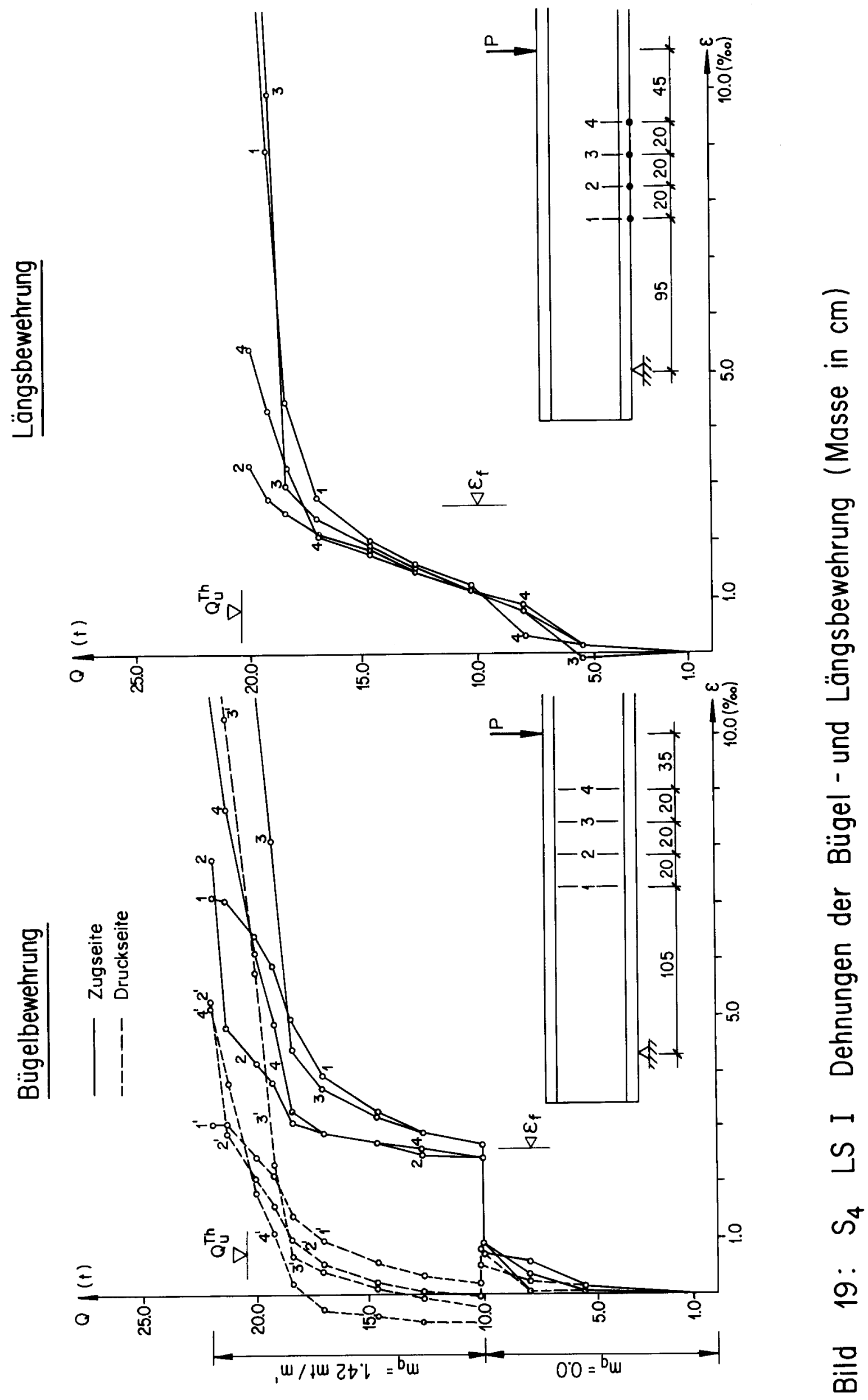




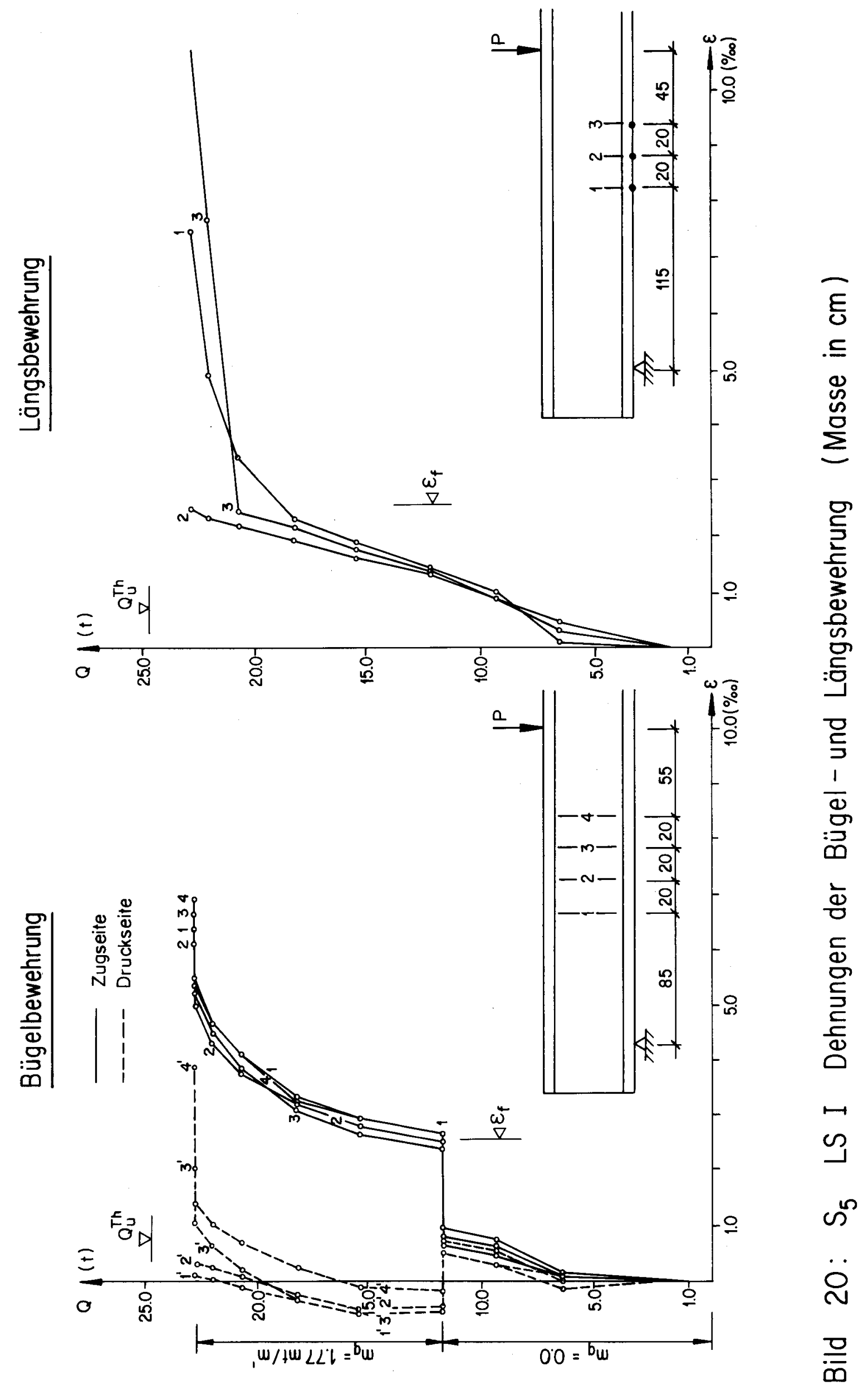




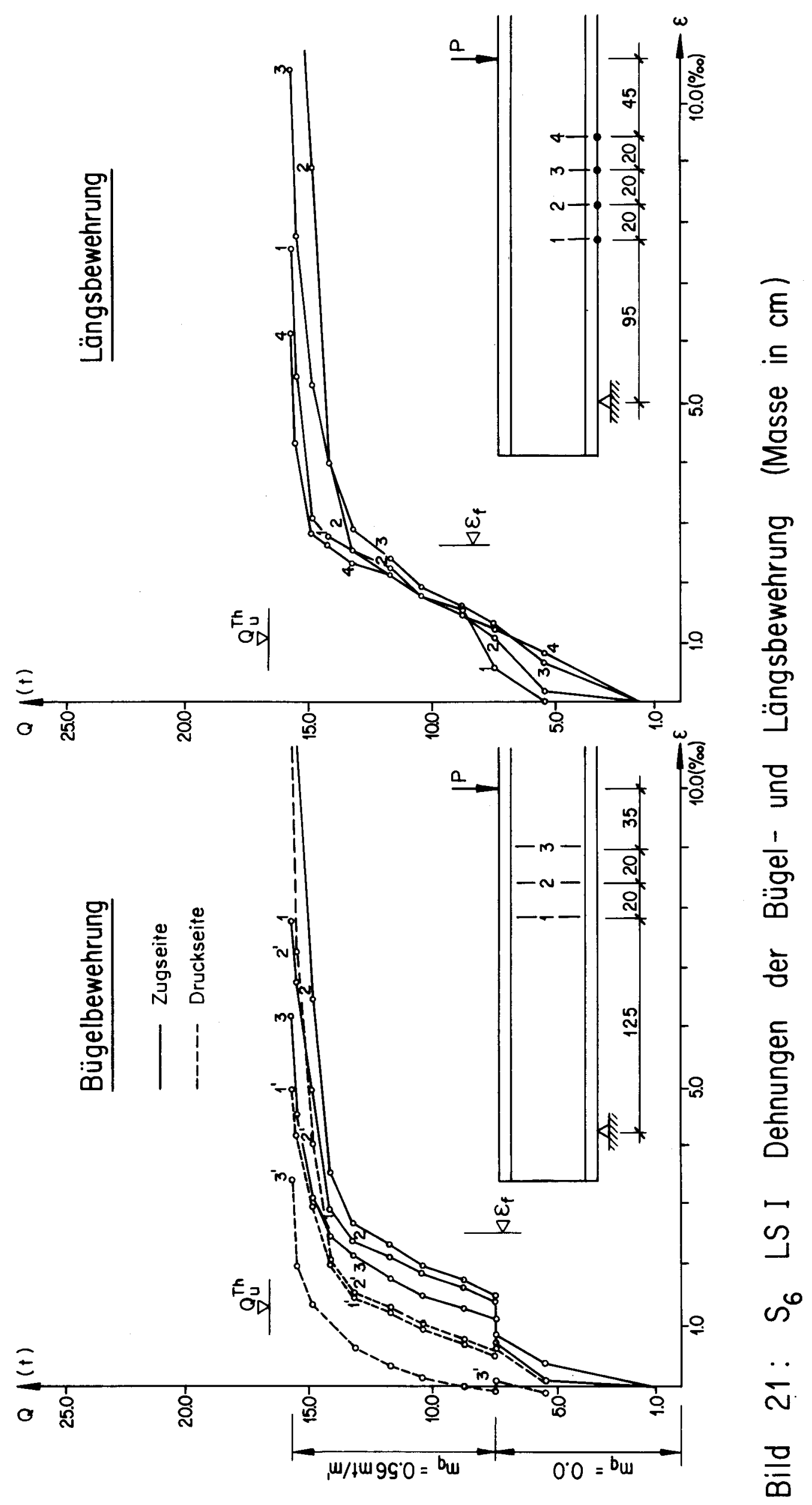




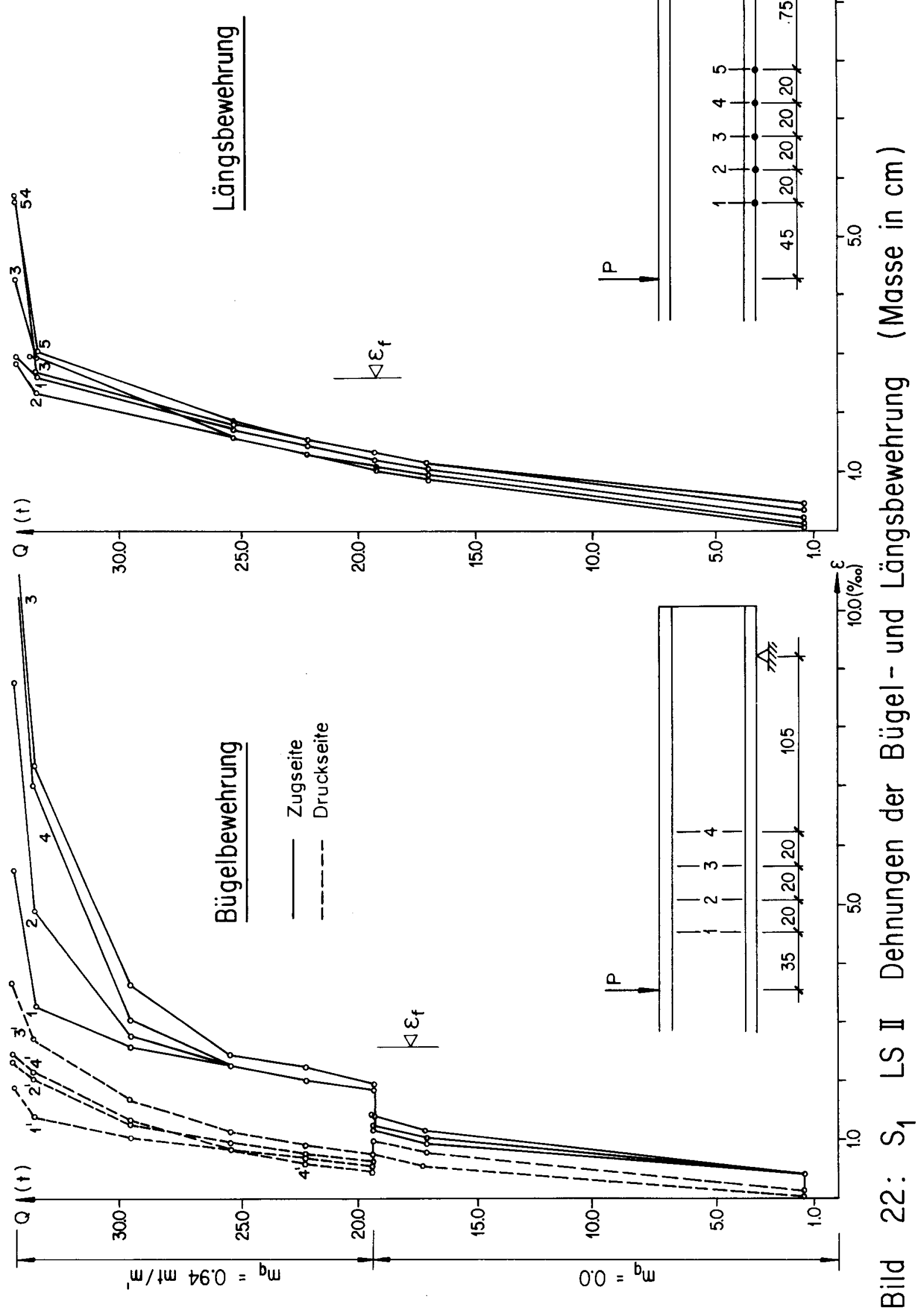




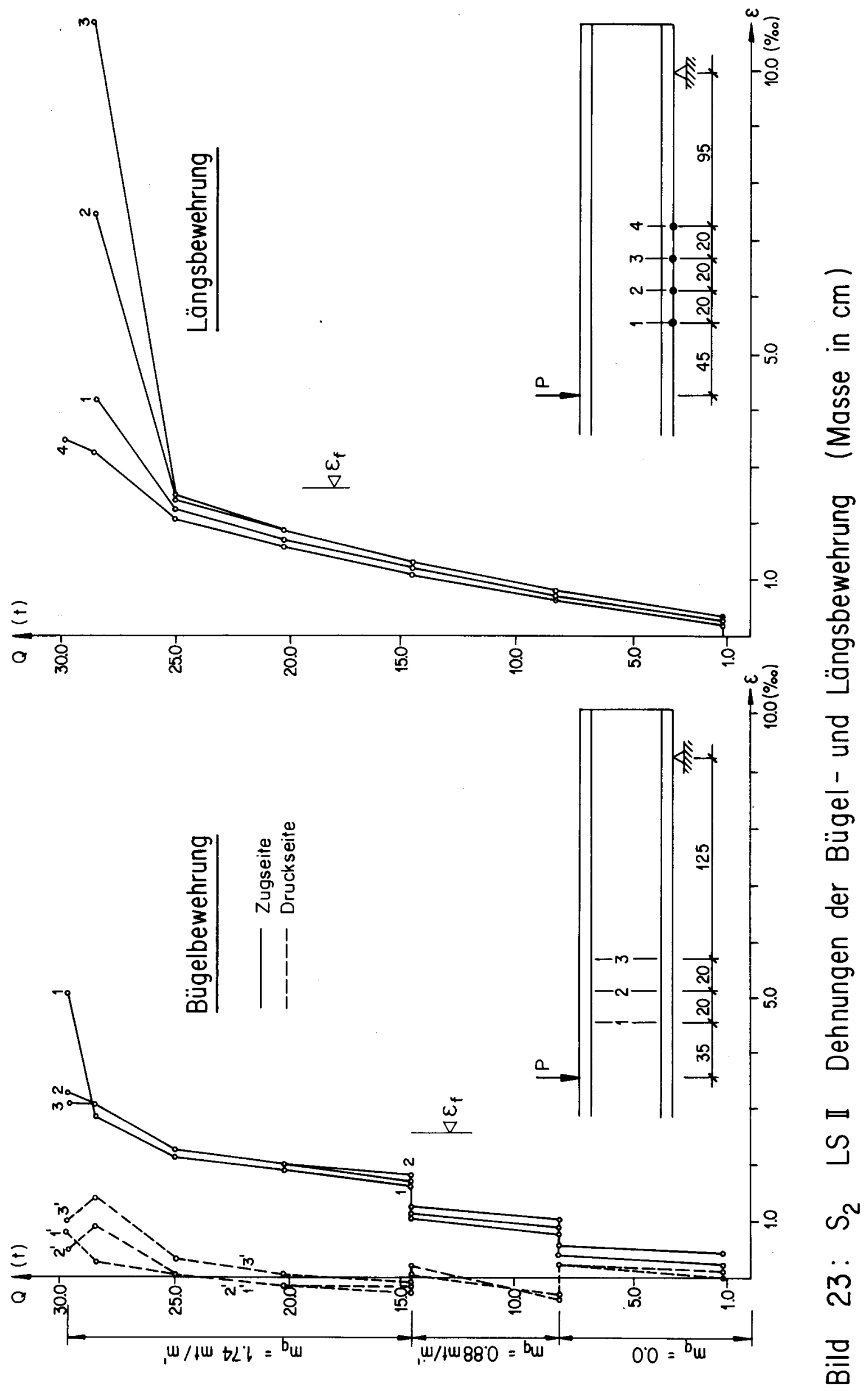




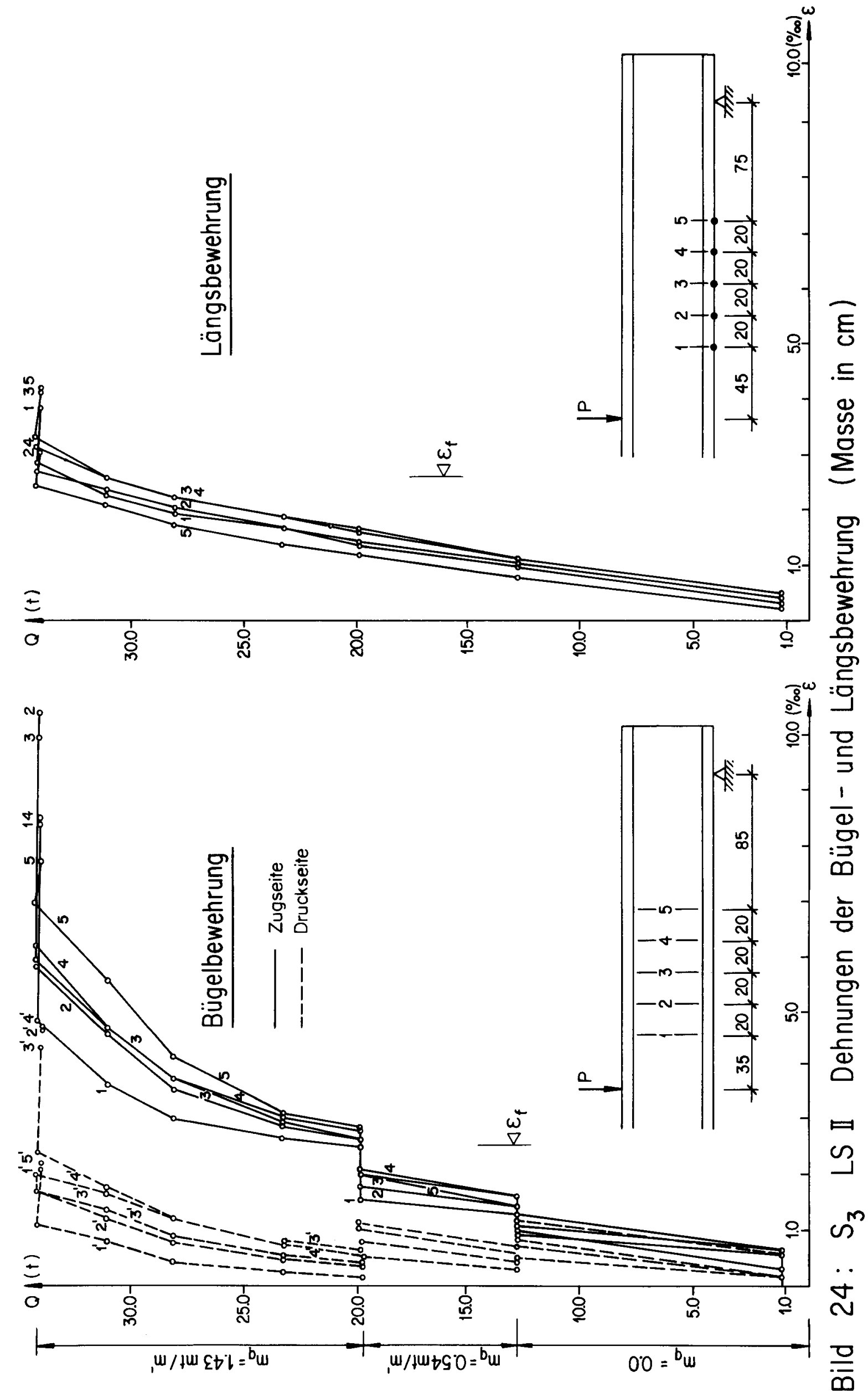




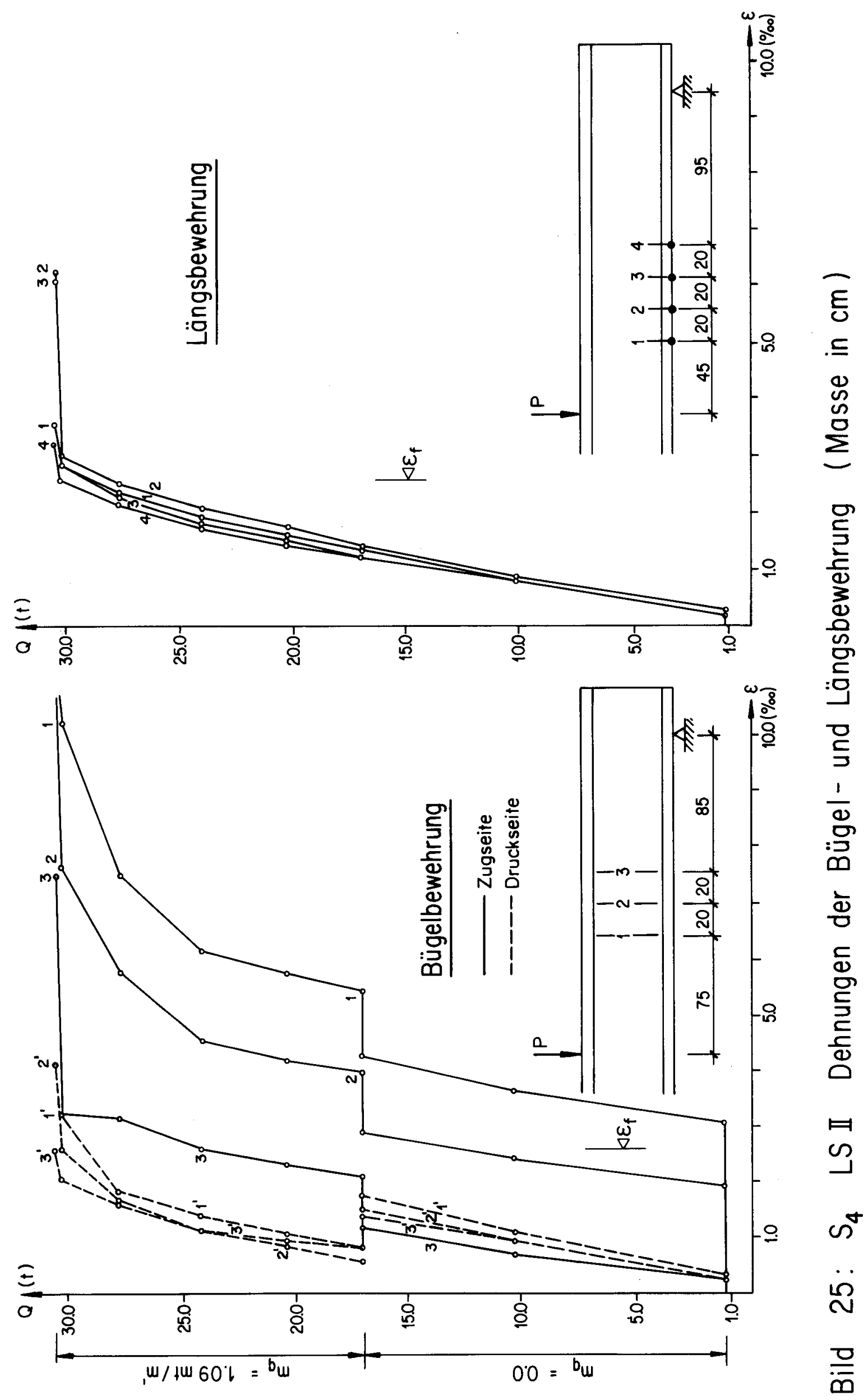




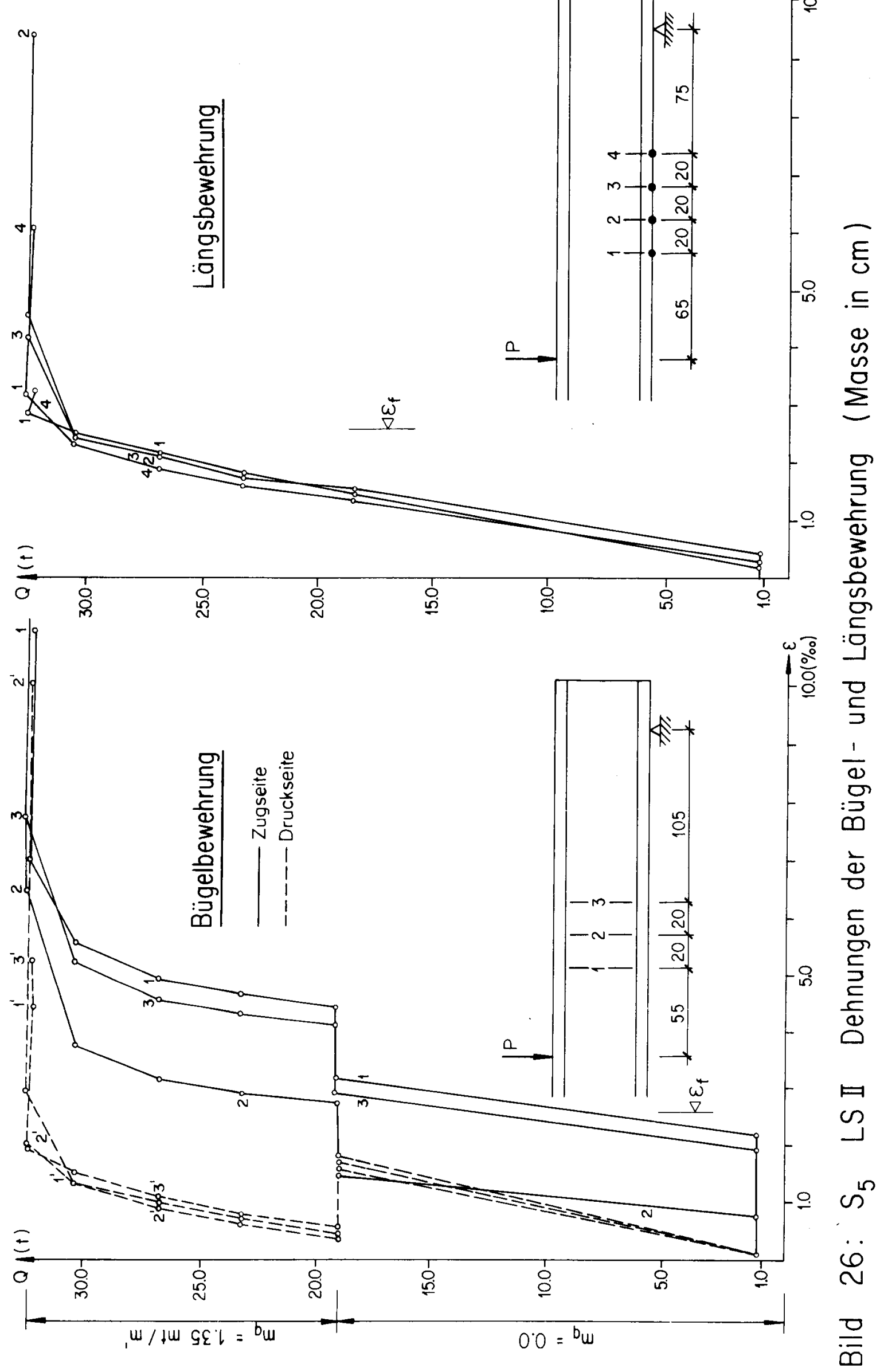




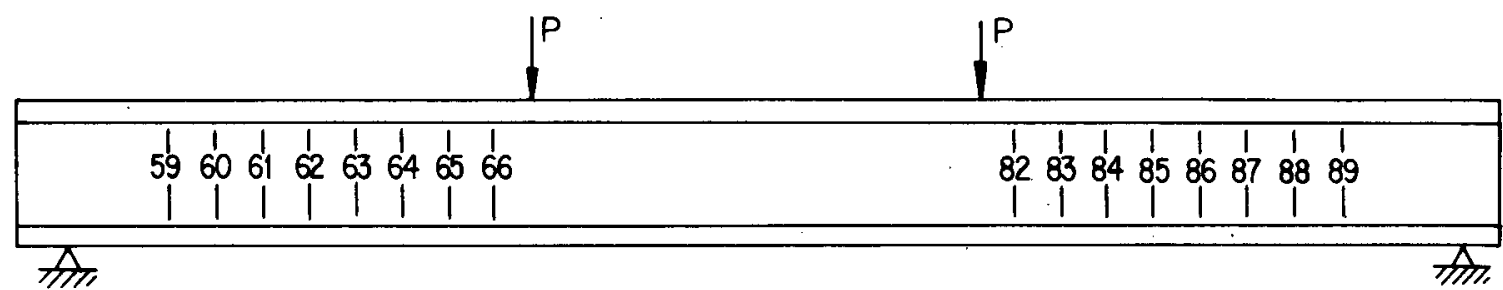
Loststellung I
Loststellung II

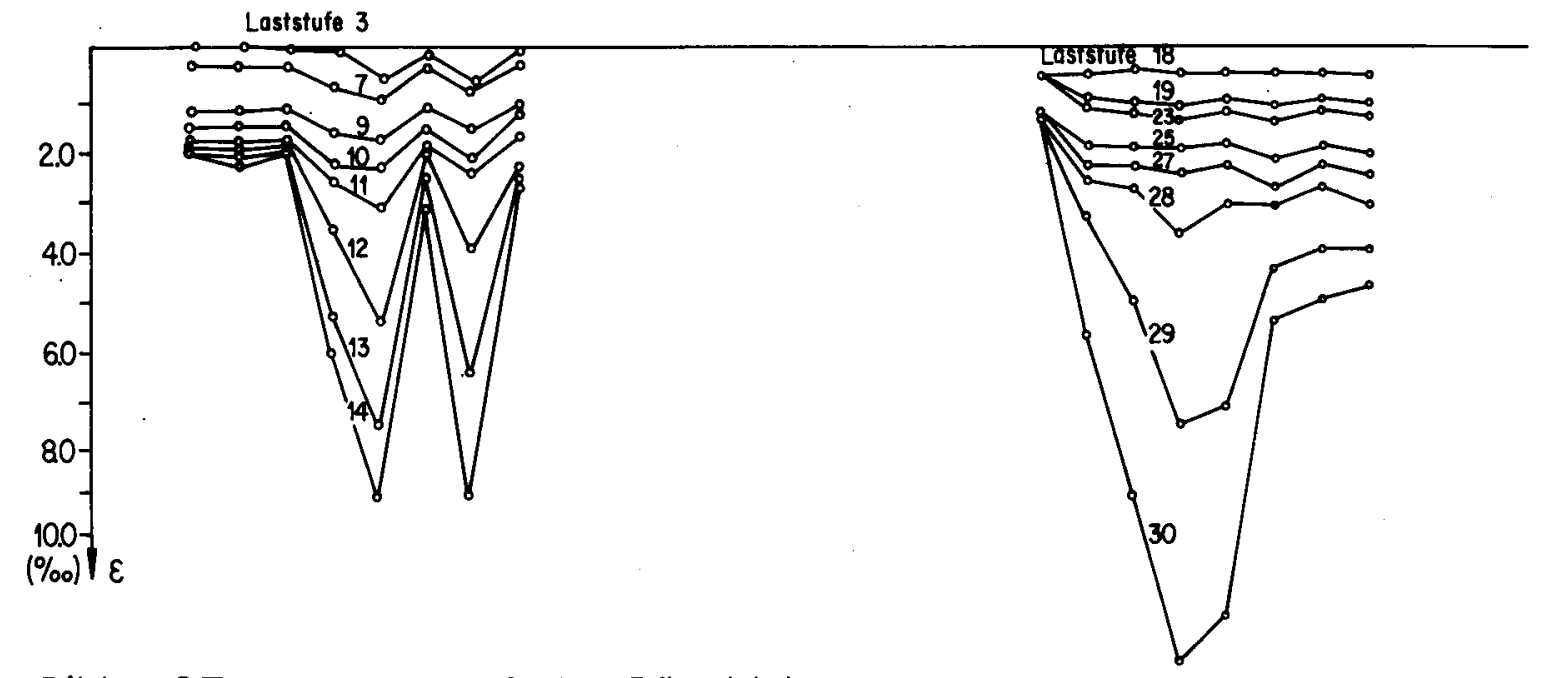

Bild 27: $S_{1}$ Verlauf der Bügeldehnungen
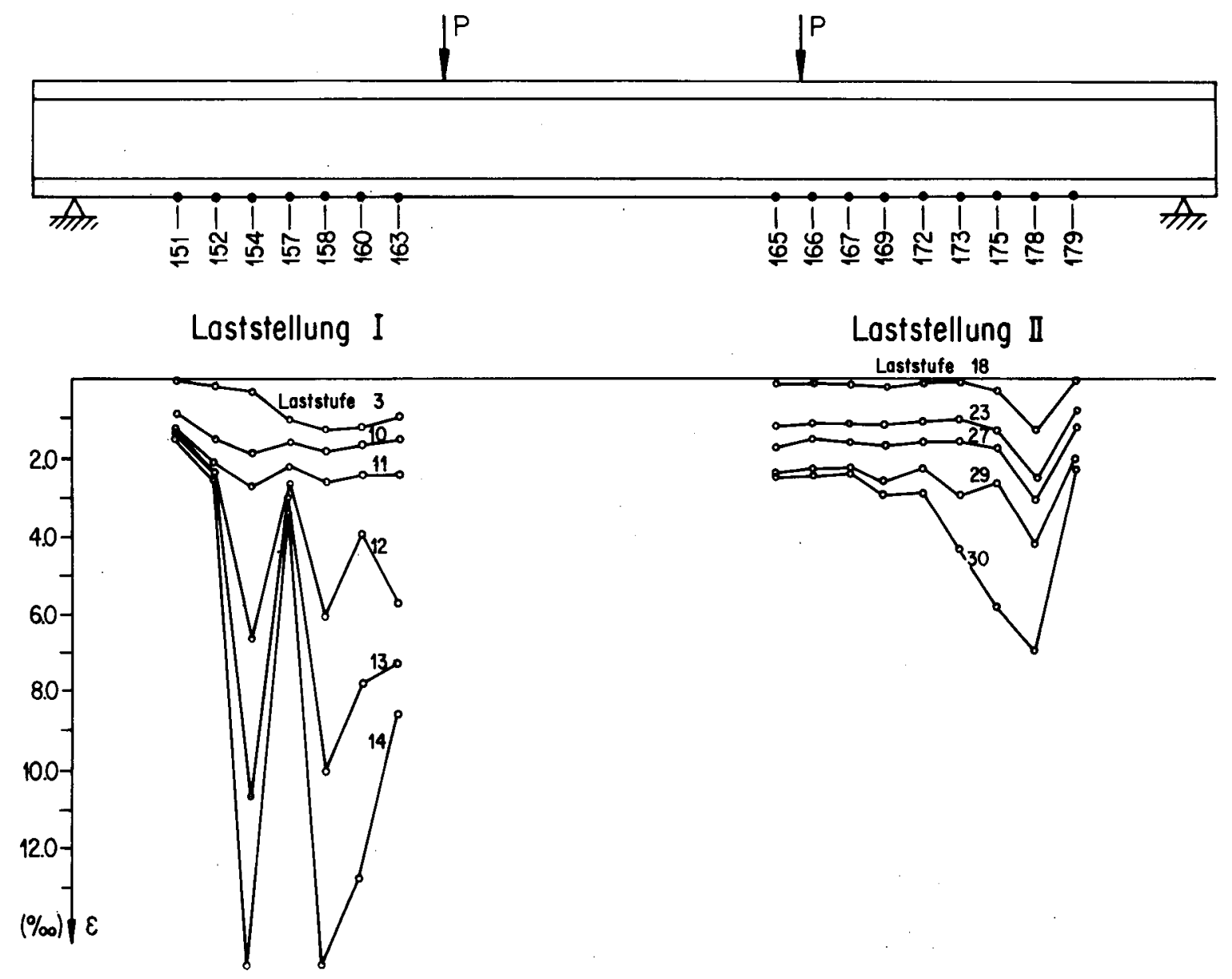

Bild 28: $S_{1}$ Dehnungsverlauf der Längsbewehrung 


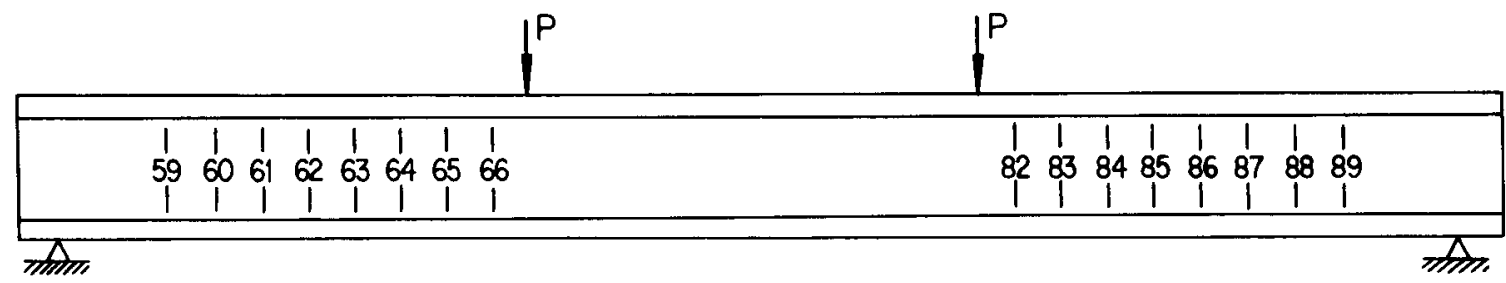

Loststellung I

Loststellung II

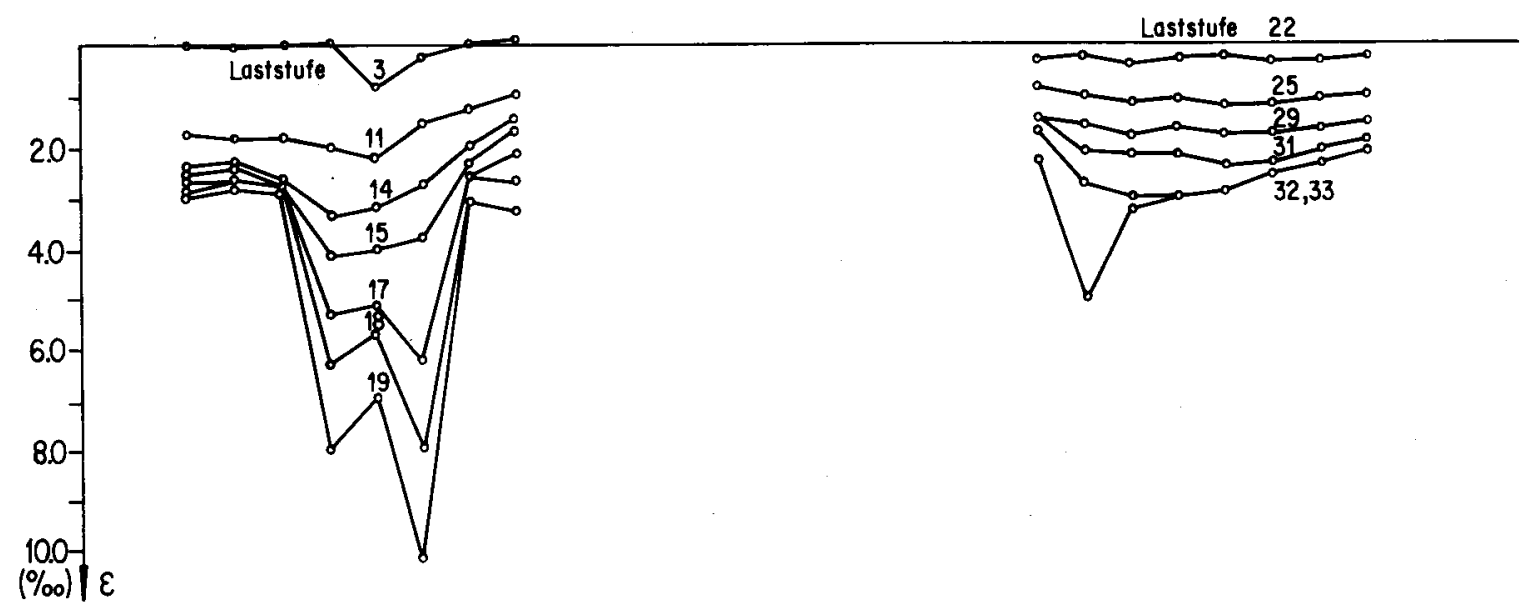

Bild 29: $S_{2}$ Verlauf der Bügeldehnungen

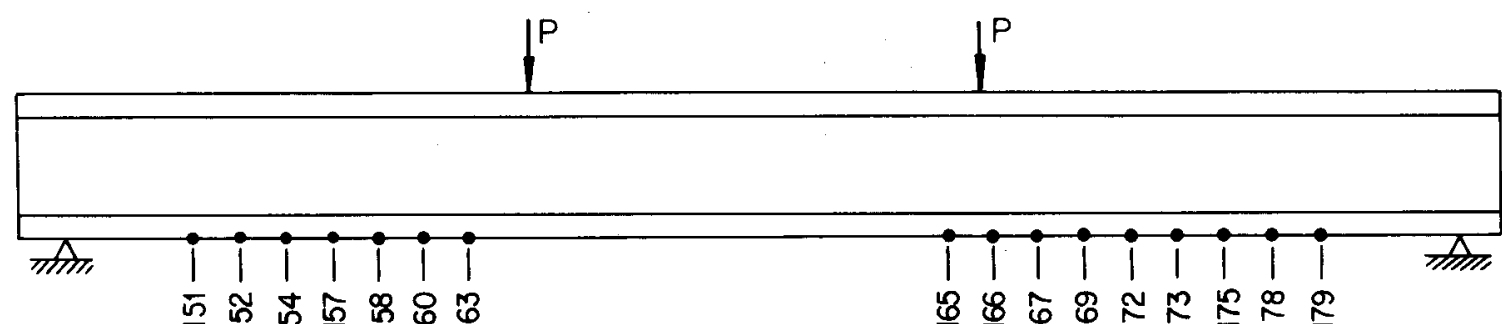

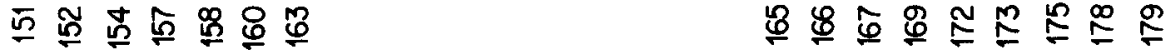

Laststellung I Loststellung II
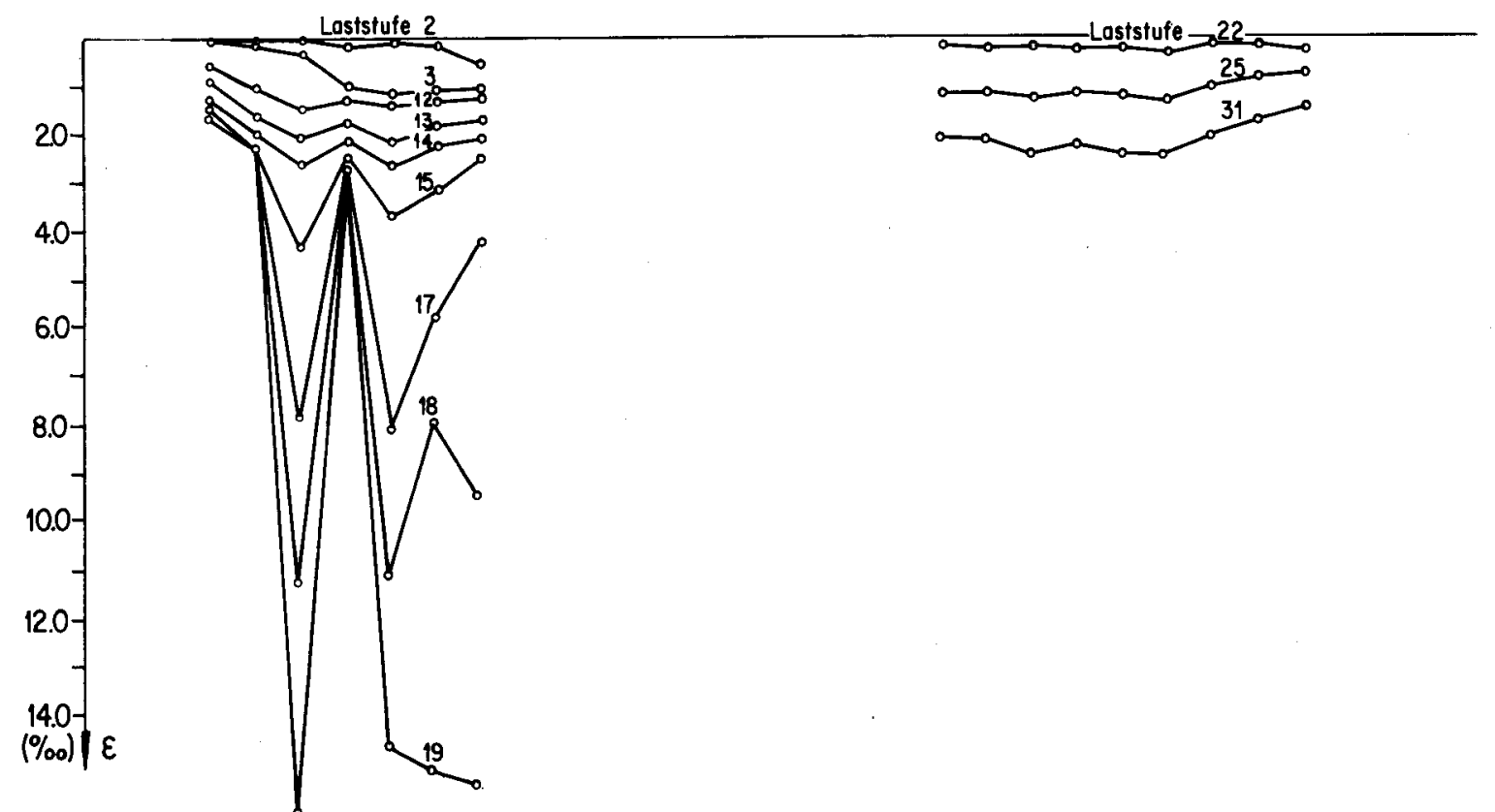

Bild 30: $\mathrm{S}_{2}$ Dehnungsverlauf der Längsbewehrung 

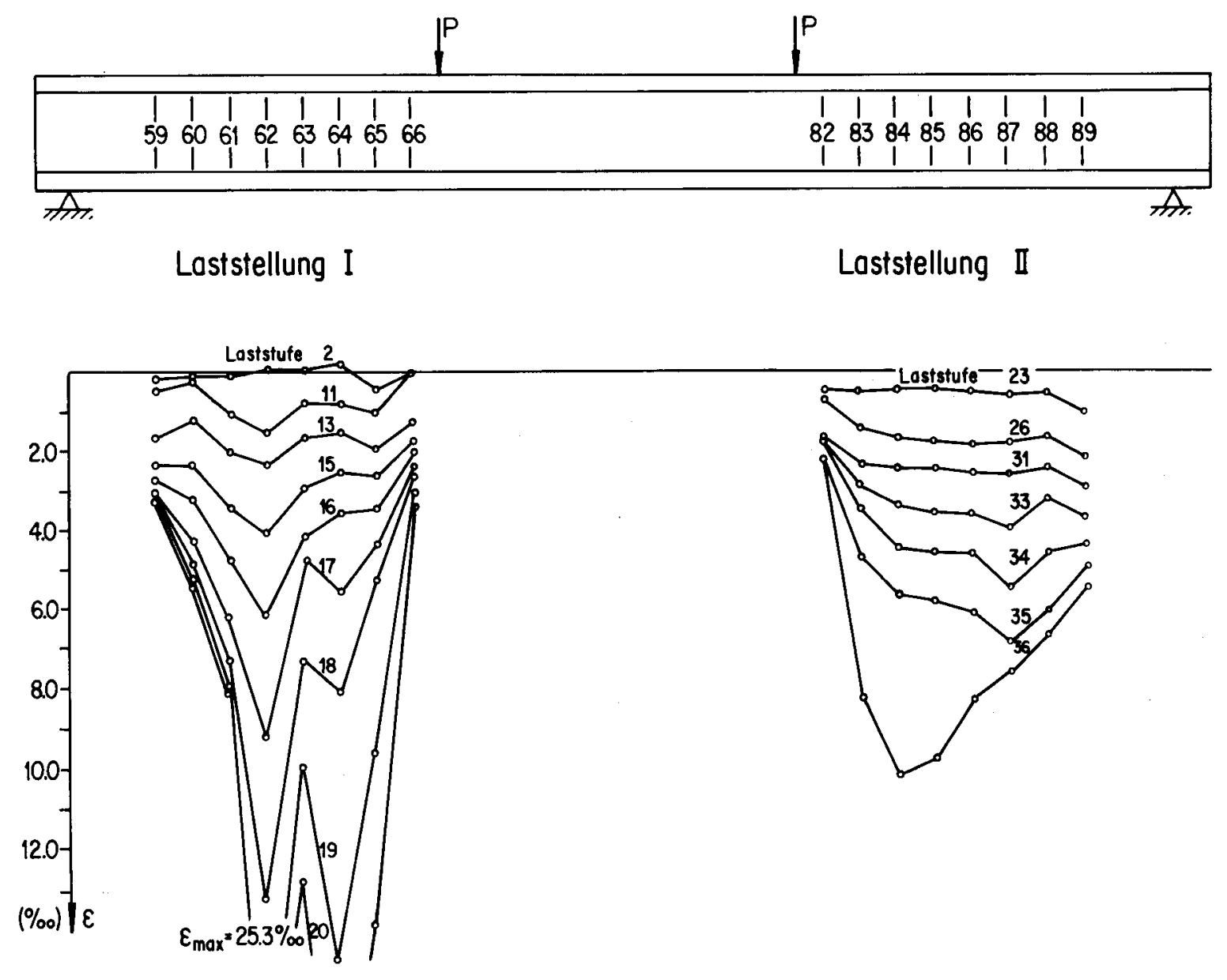

Bild 31: $S_{3}$ Verlauf der Bügeldehnungen

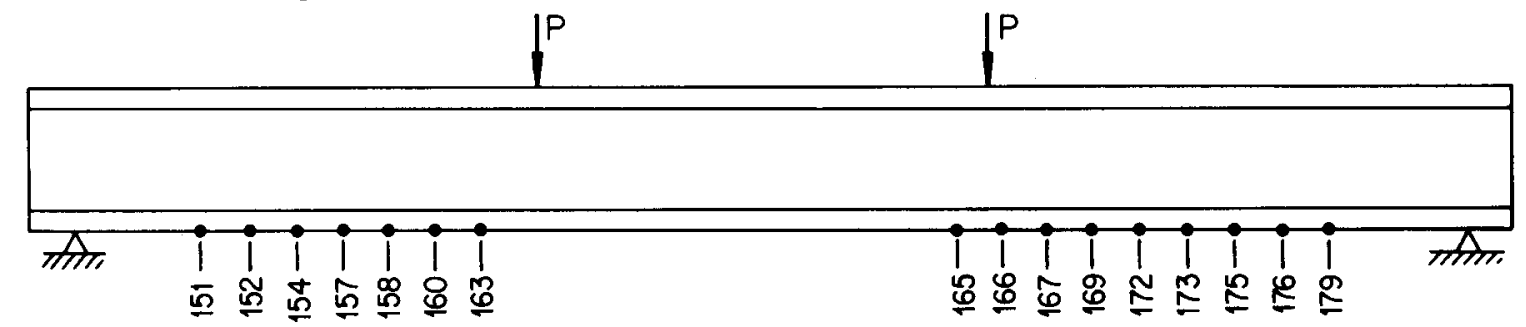
Laststellung I Laststellung II

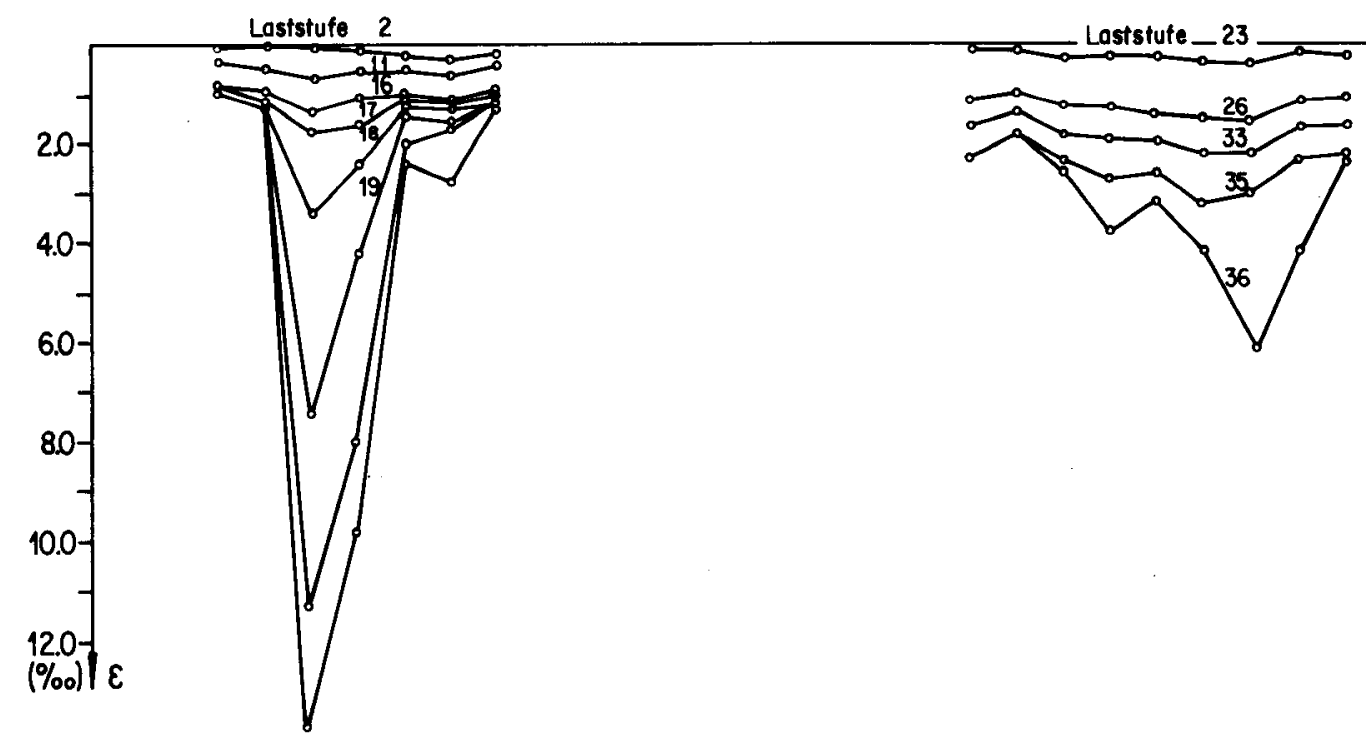

Bild 32: $S_{3}$ Dehnungsverlauf der Längsbewehrung 


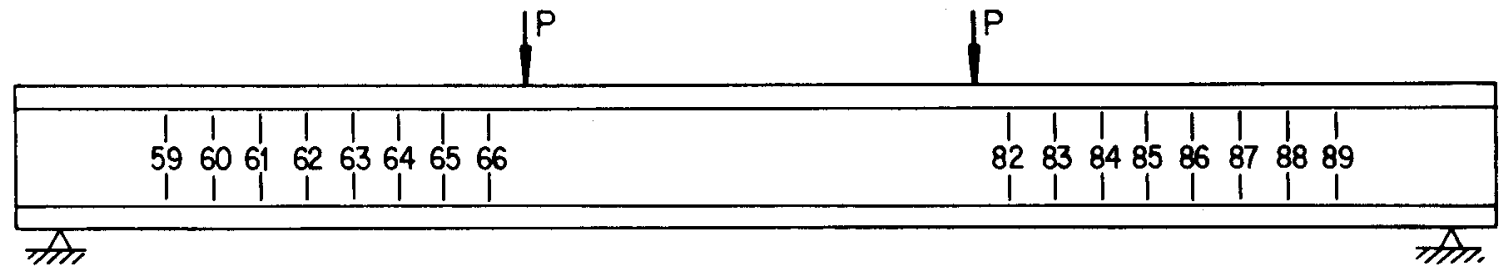

Loststellung I Laststellung II

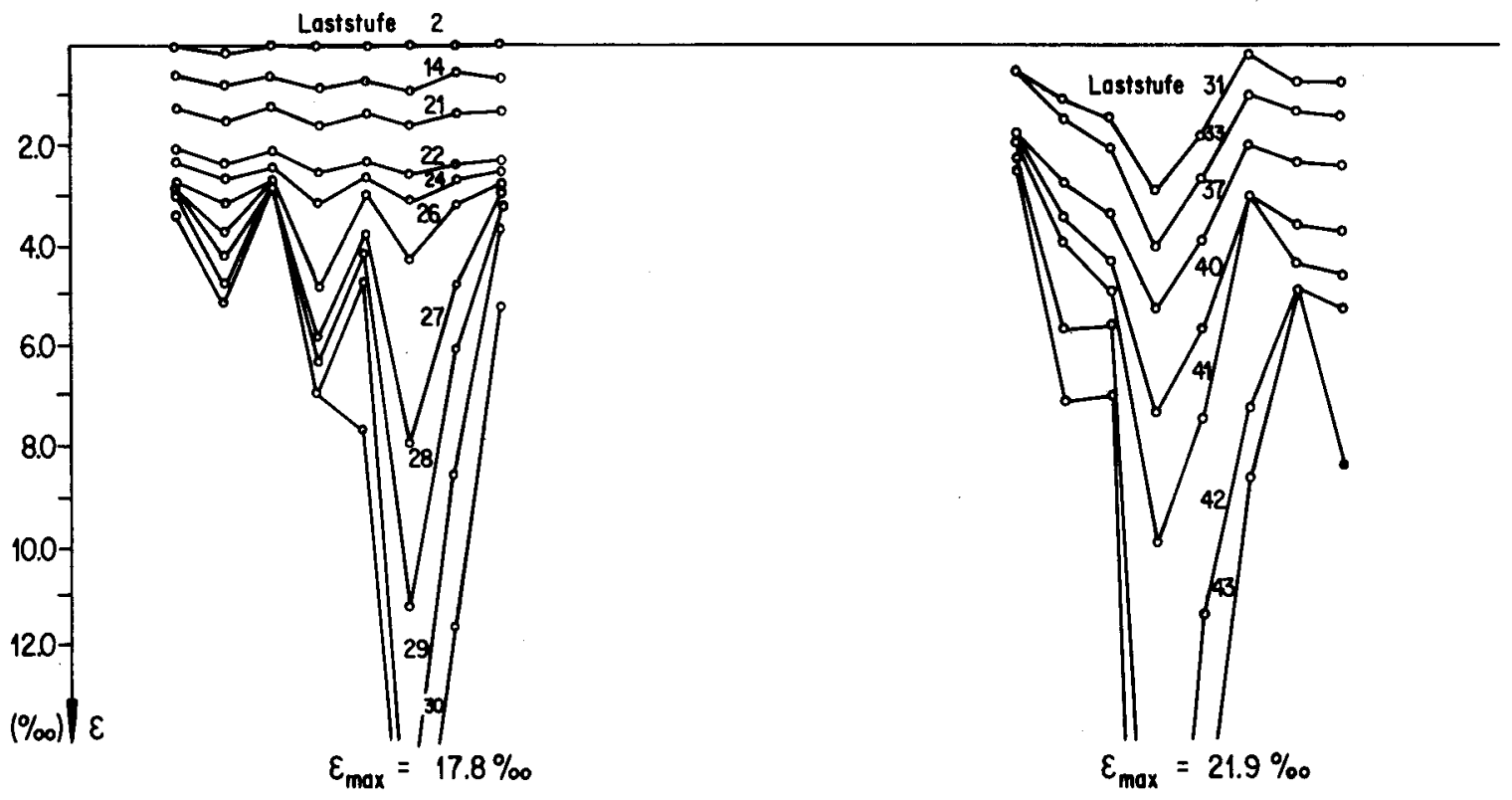

Bild 33: $S_{4}$ Verlauf der Bügeldehnungen

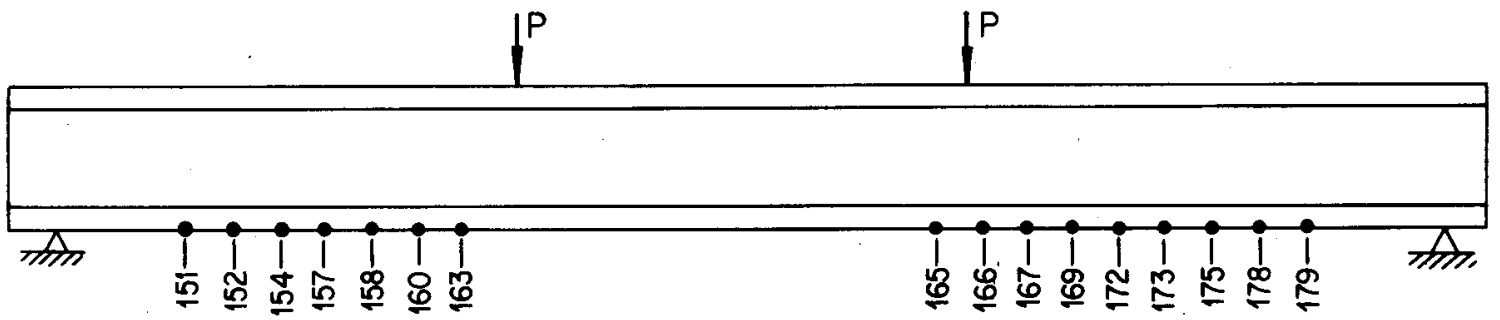

Loststellung I

Loststellung II

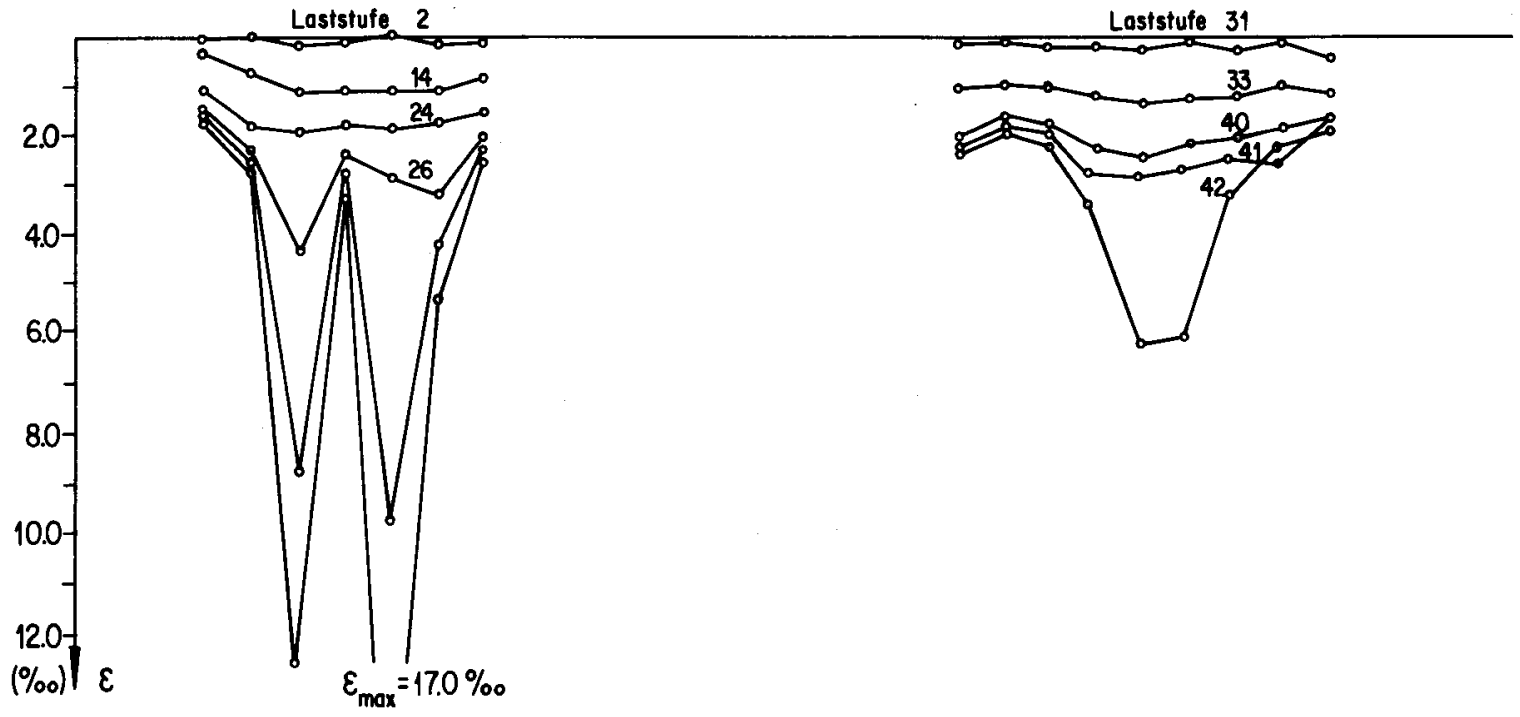

Bild 34: $\mathrm{S}_{4}$ Dehnungsverlauf der Längsbewehrung 

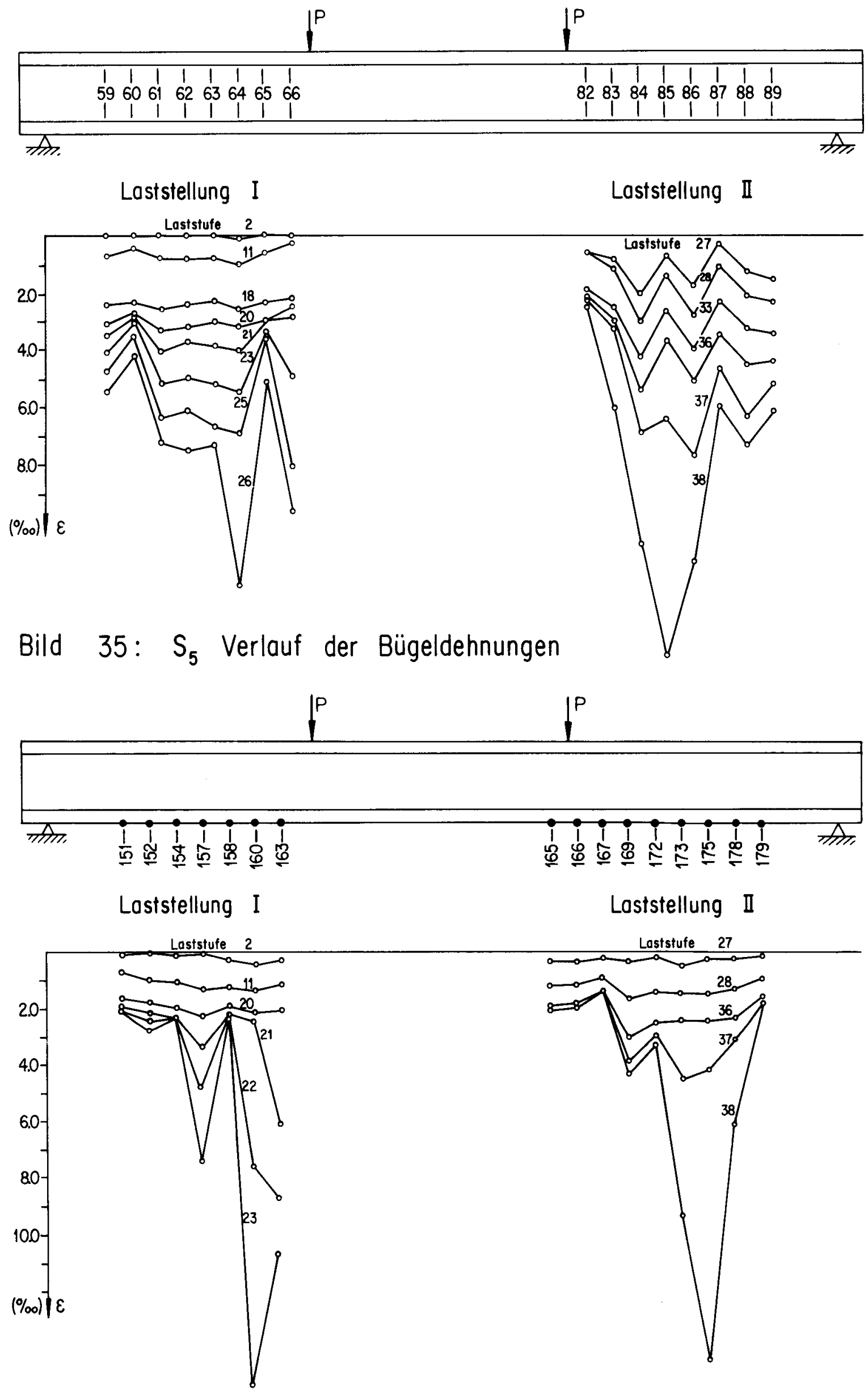

Bild 36: $\mathrm{S}_{5}$ Dehnungsverlauf der Längsbewehrung 


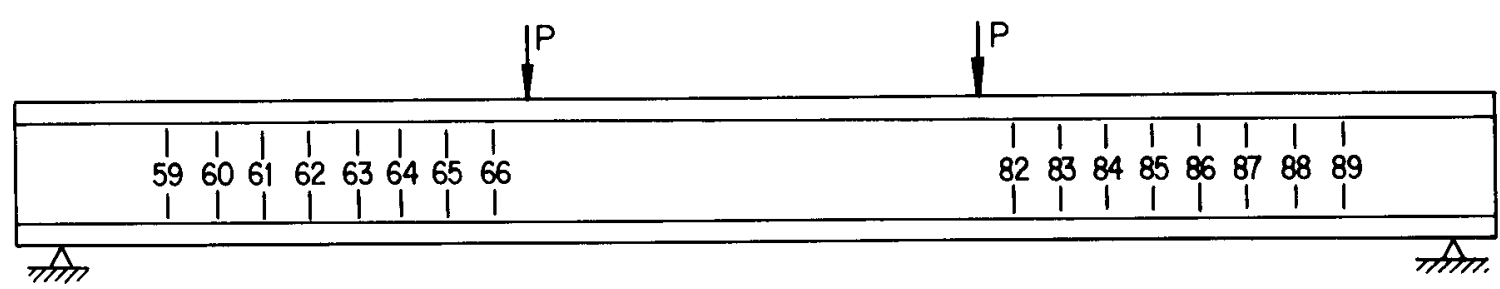
Loststellung I
Loststellung II

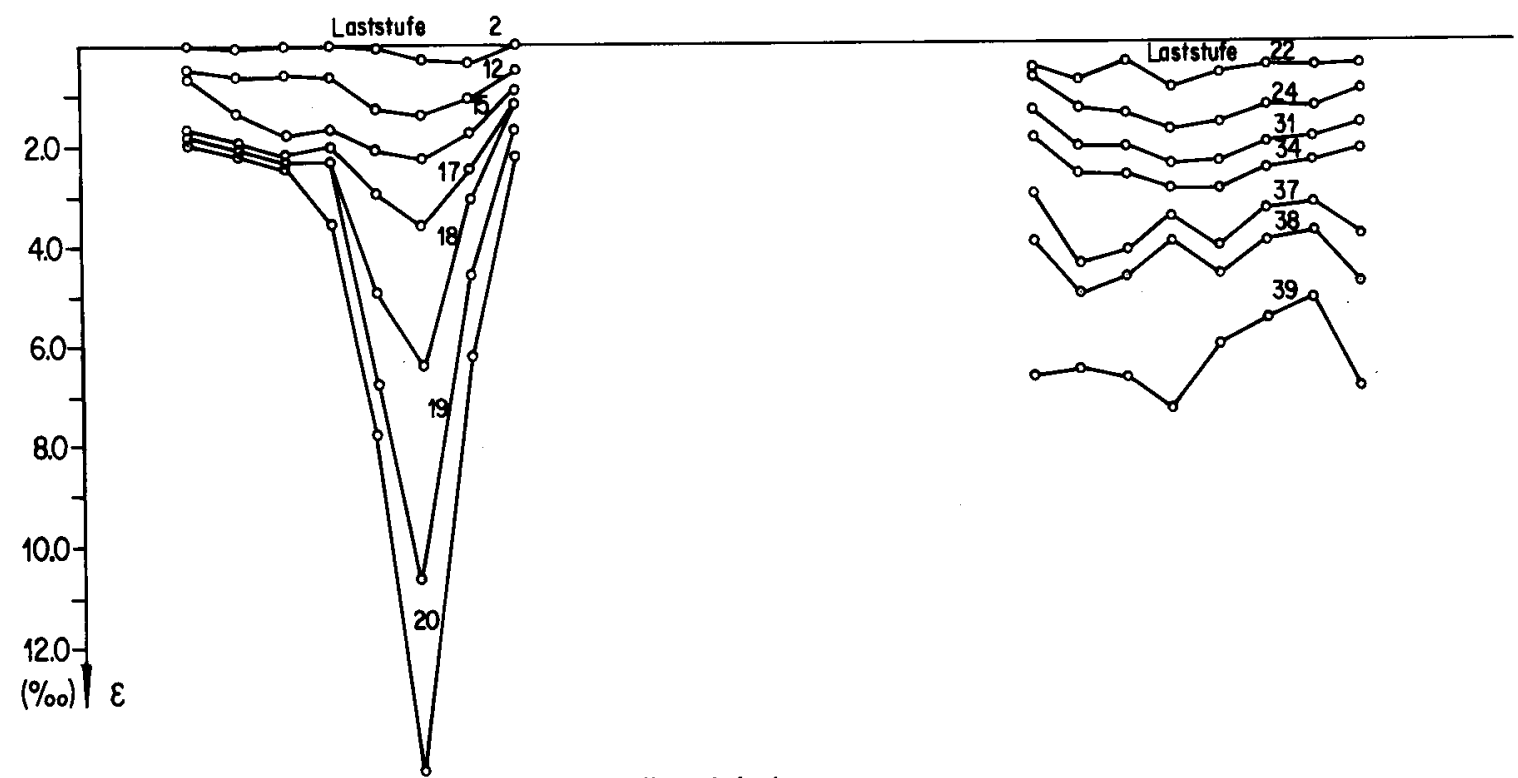

Bild 37: $S_{6}$ Verlauf der Bügeldehnungen

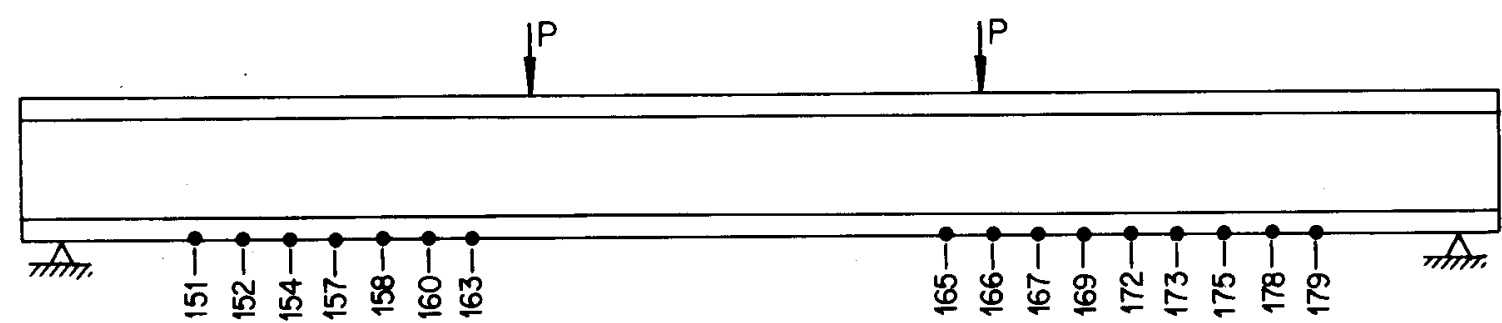

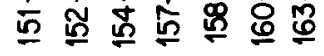

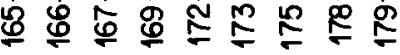

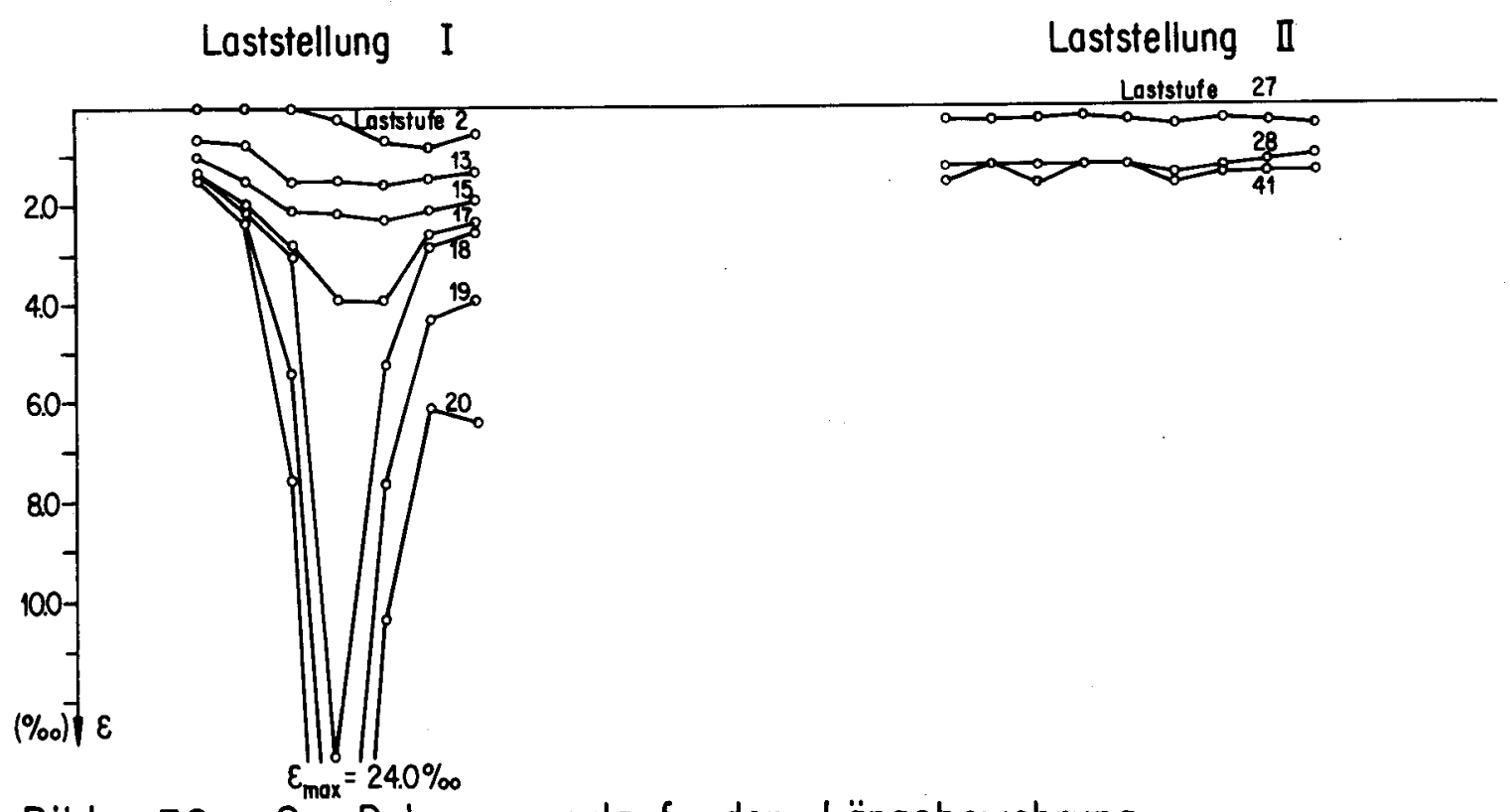

Bild 38: $\mathrm{S}_{6}$ Dehnungsverlauf der Längsbewehrung 

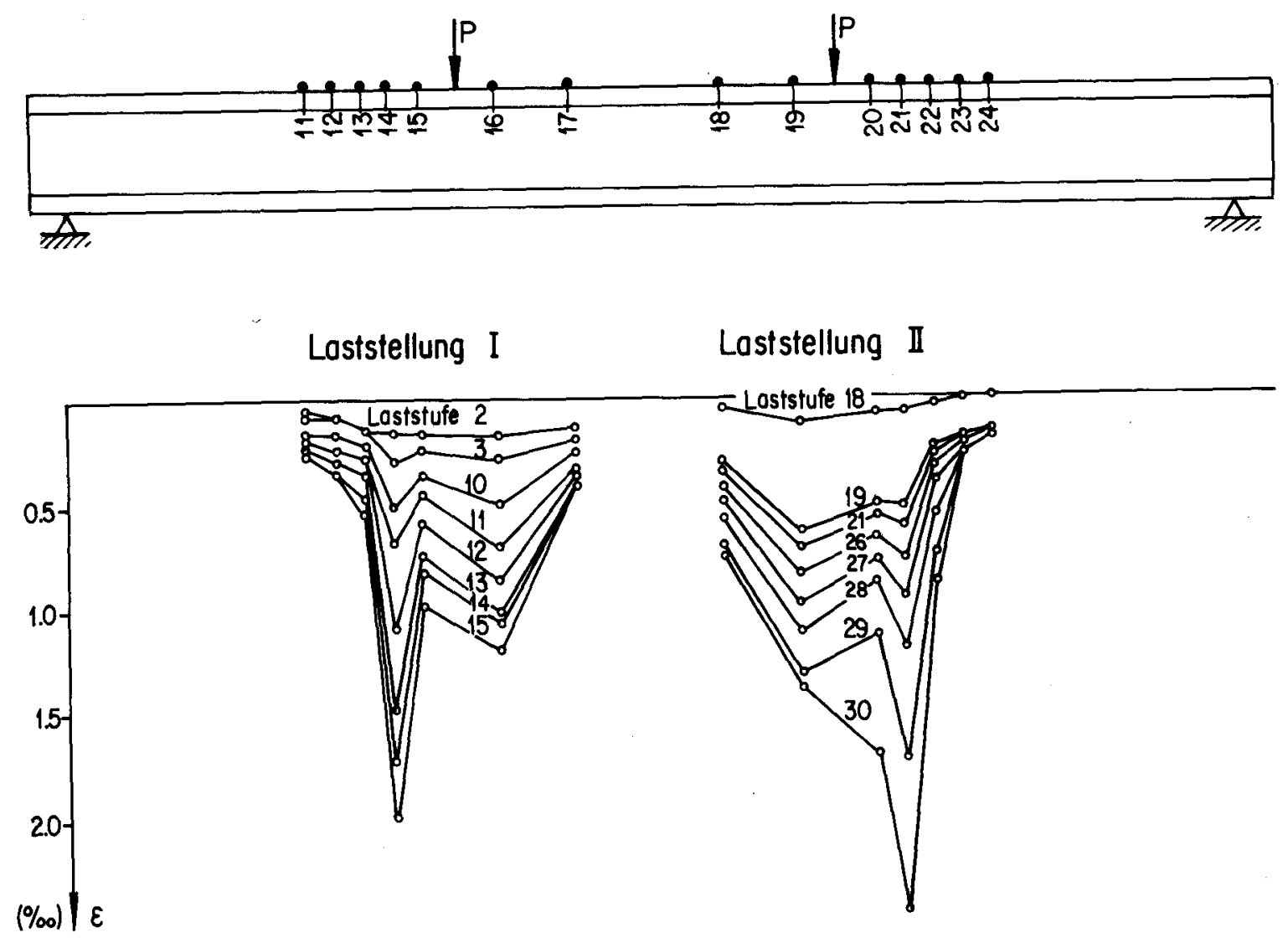

Bild 39: $S_{1}$ Betonstauchungen
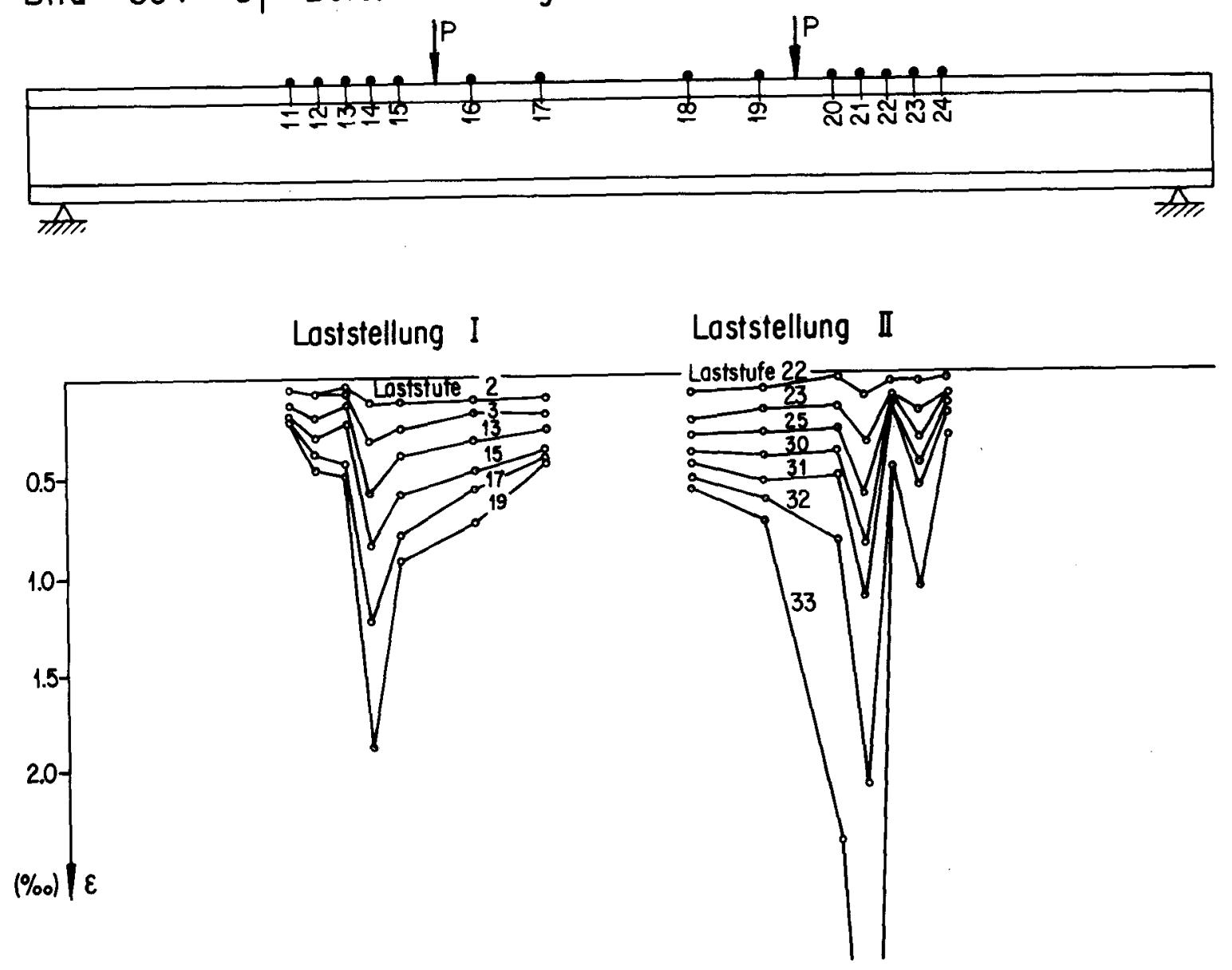

Bild 40: $S_{2}$ Betonstauchungen 

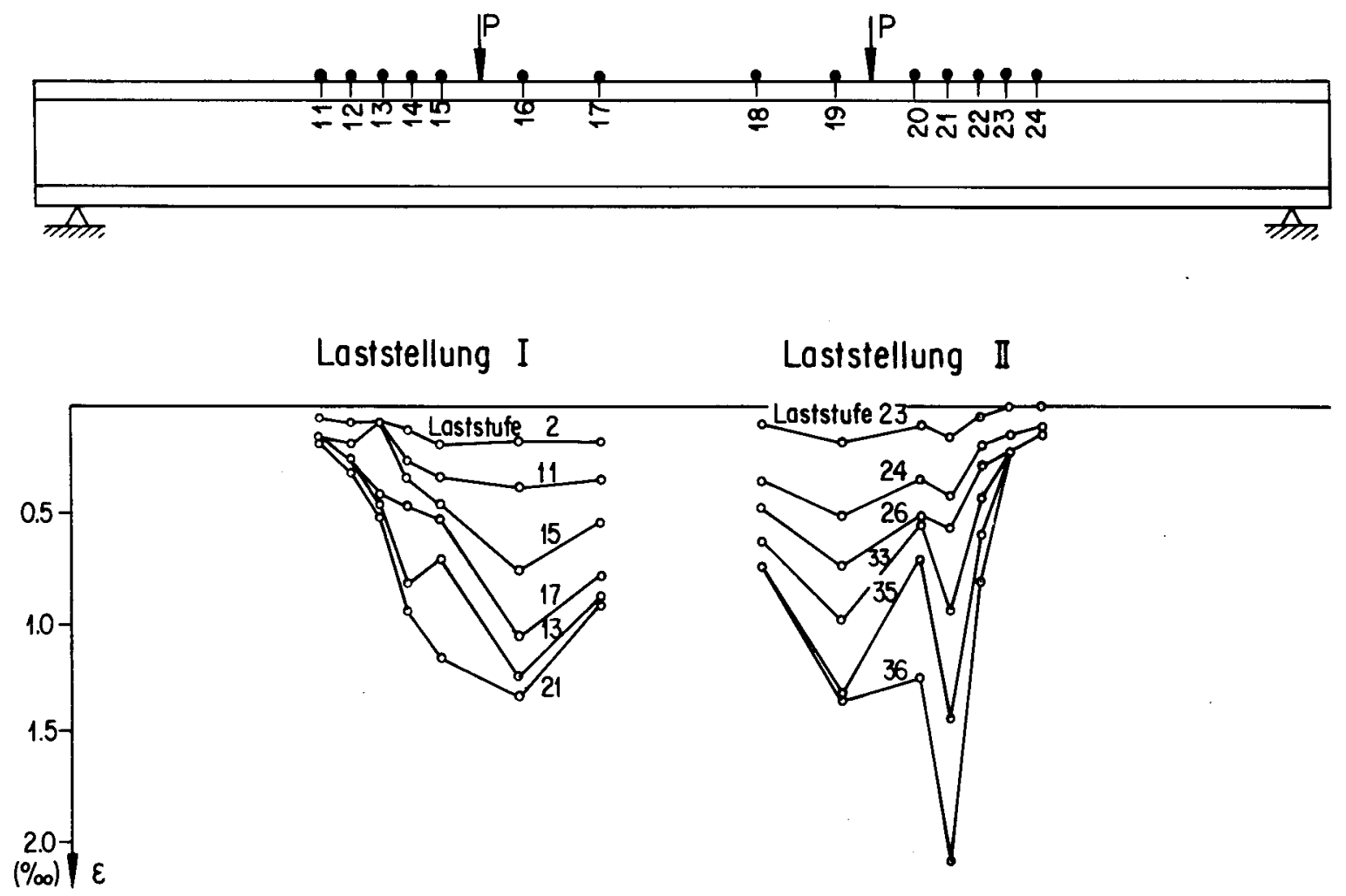

Bild 41: $S_{3}$ Betonstauchungen
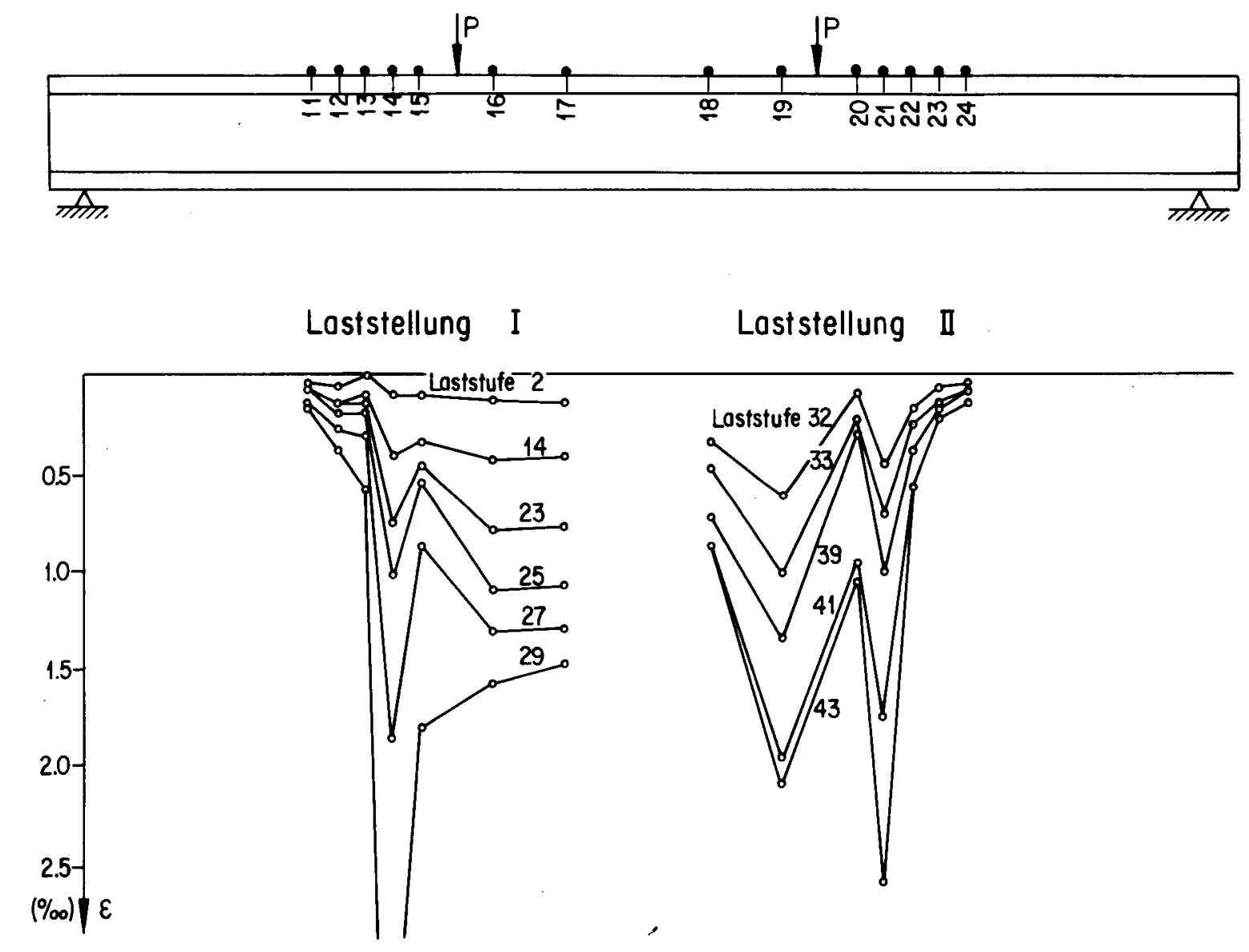

Bild 42: $\mathrm{S}_{4}$ Betonstauchungen 

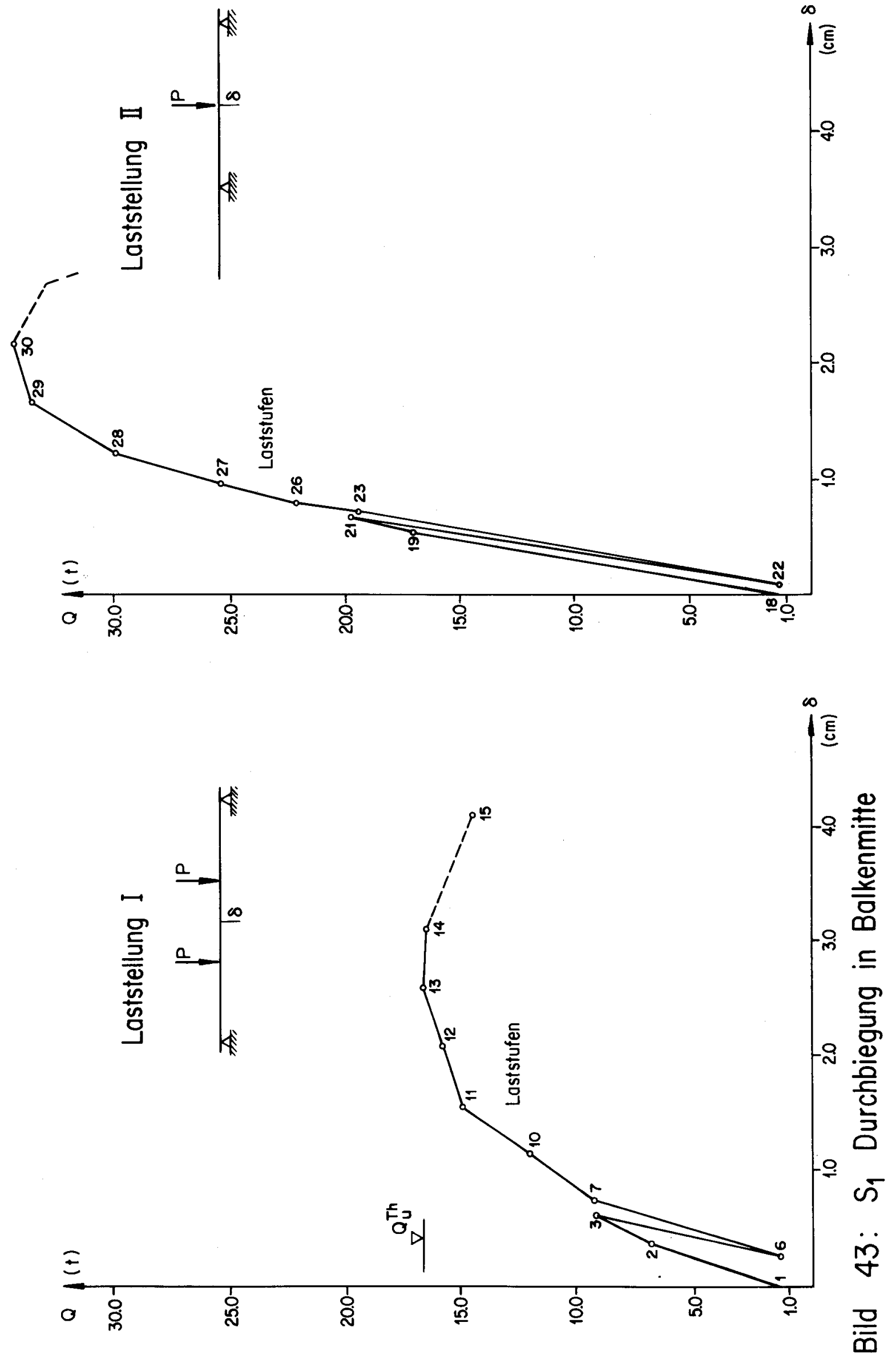

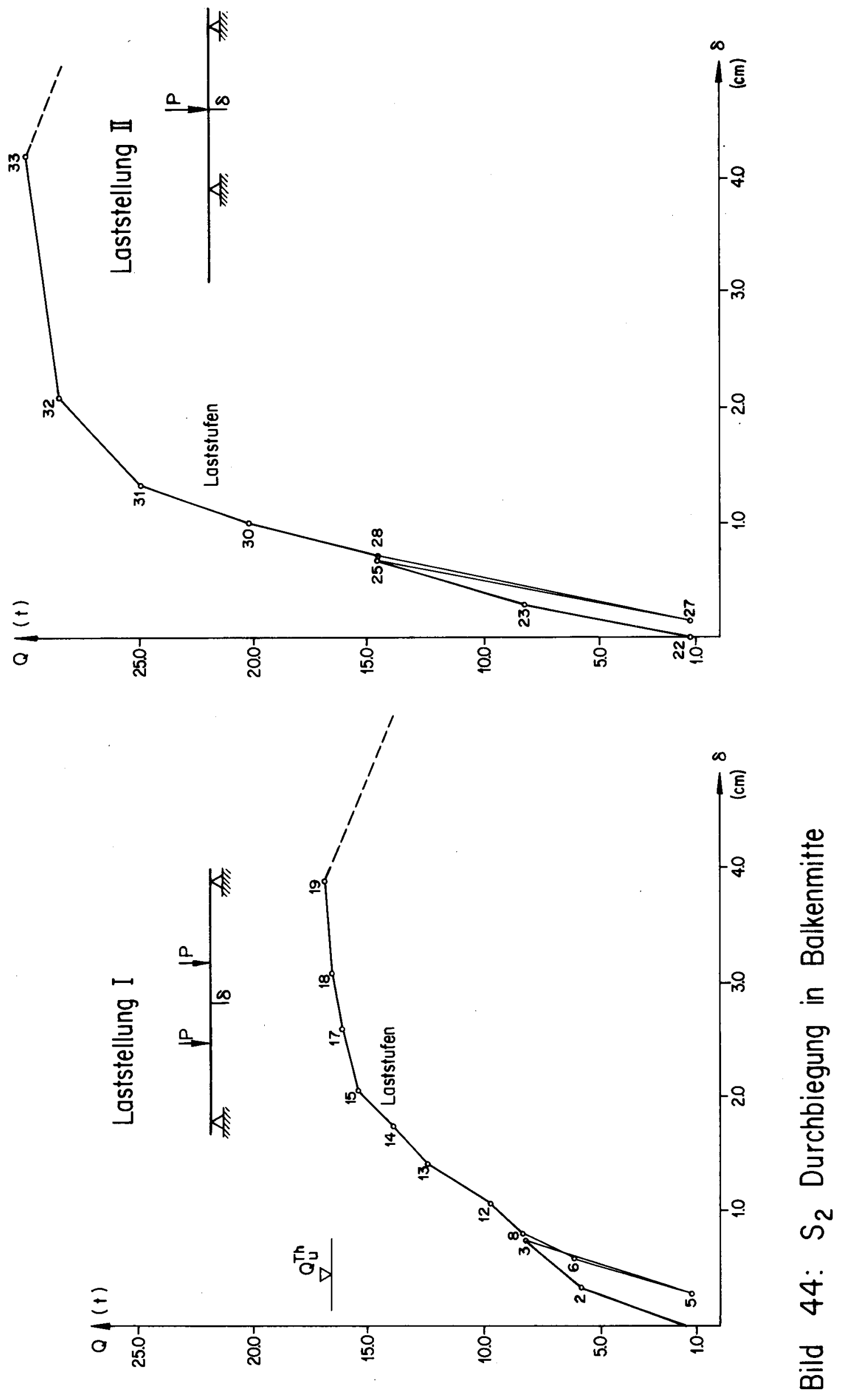


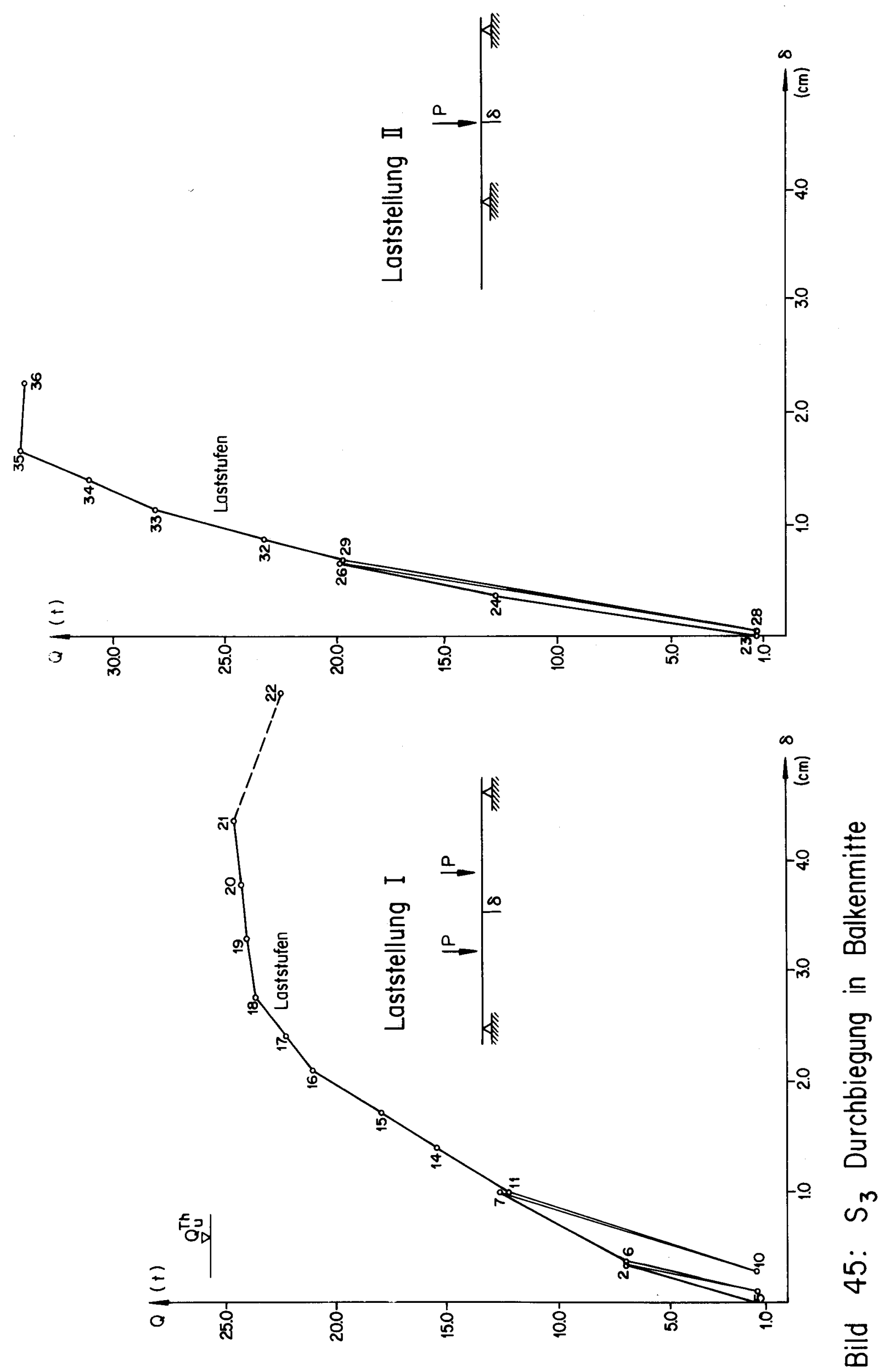




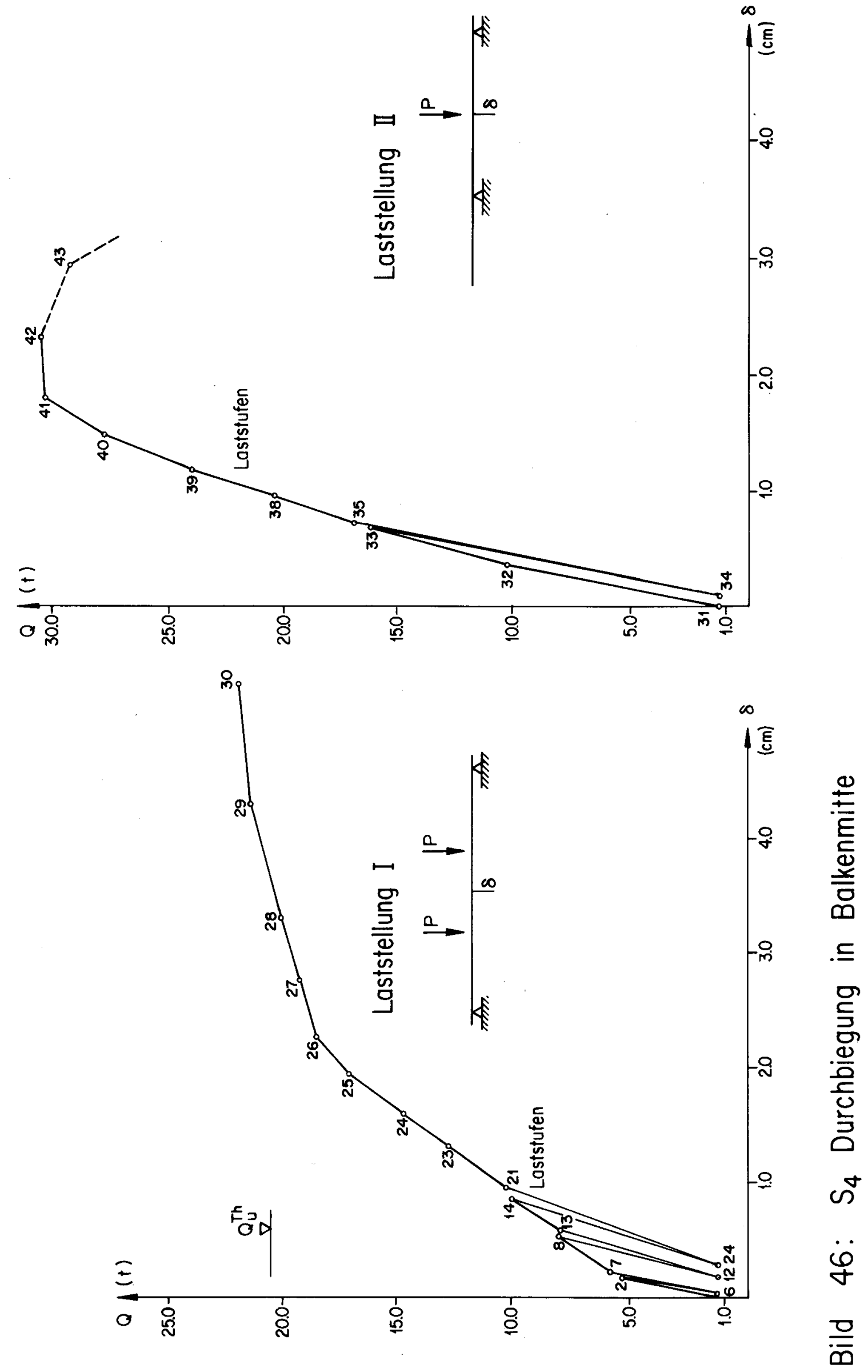




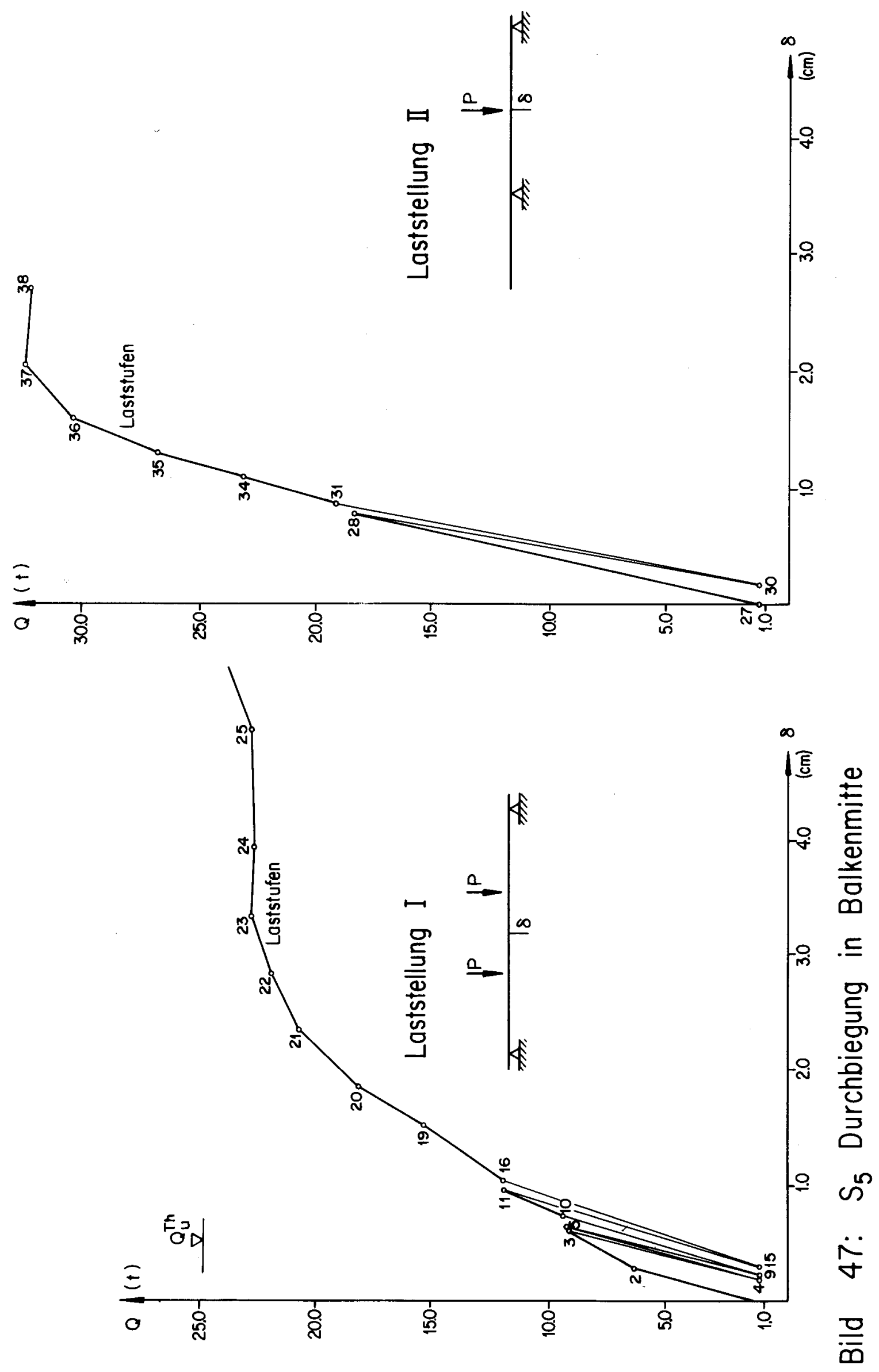



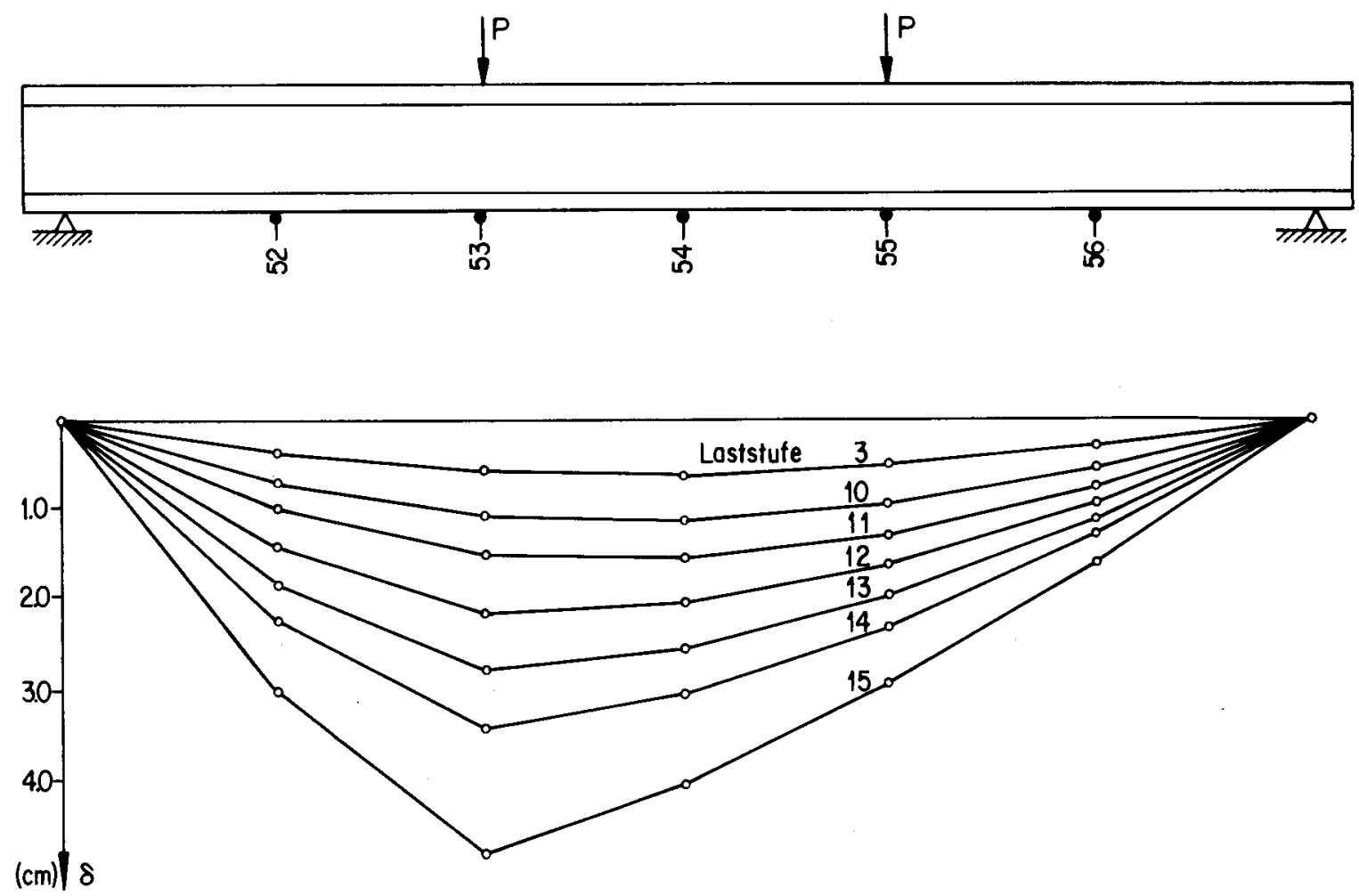

Bild 48: $S_{1}$ Durchbiegung Laststellung I
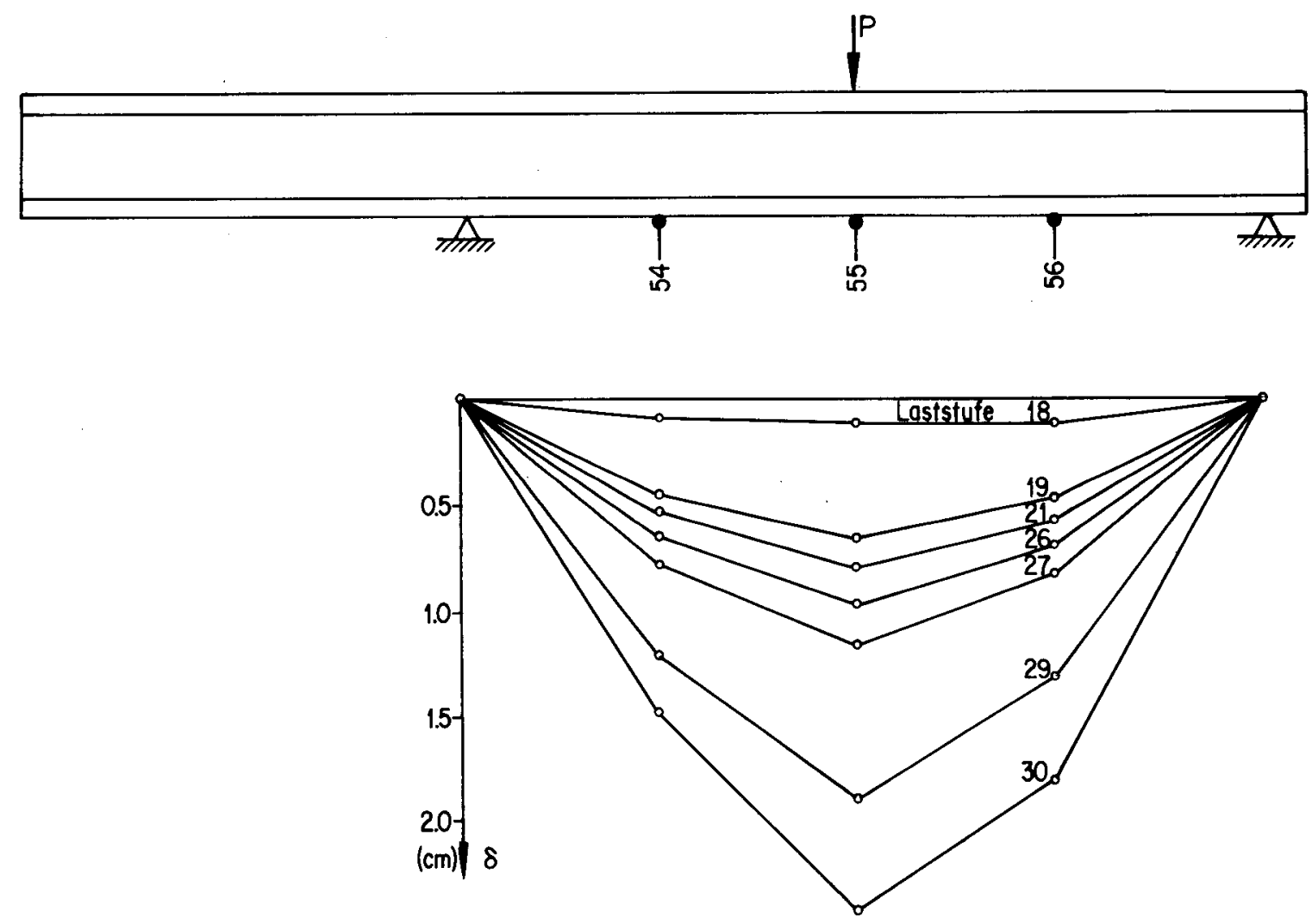

Bild 49: $S_{1}$ Durchbiegung Laststellung II 

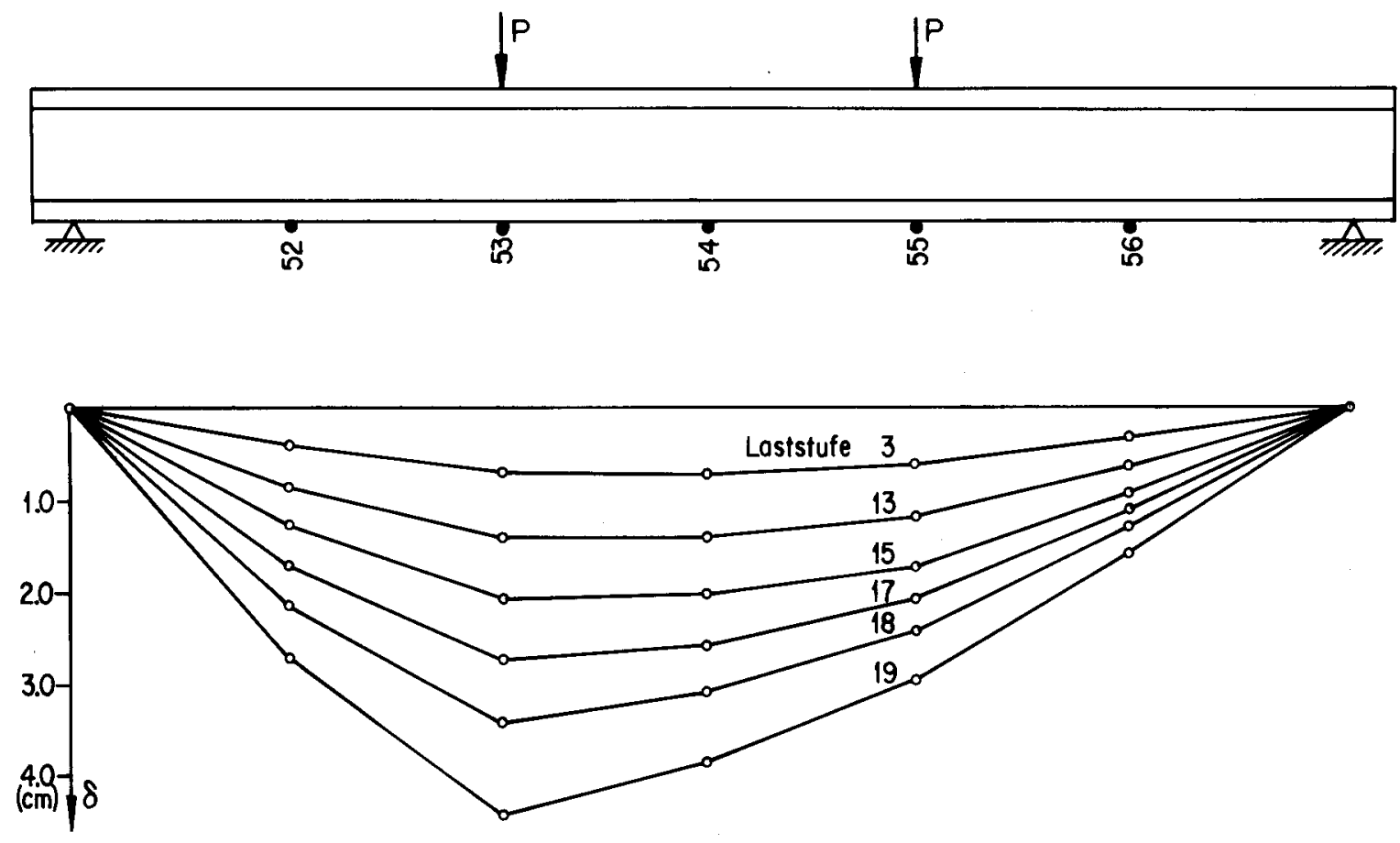

Bild 50: $\mathrm{S}_{2}$ Durchbiegung Laststellung I
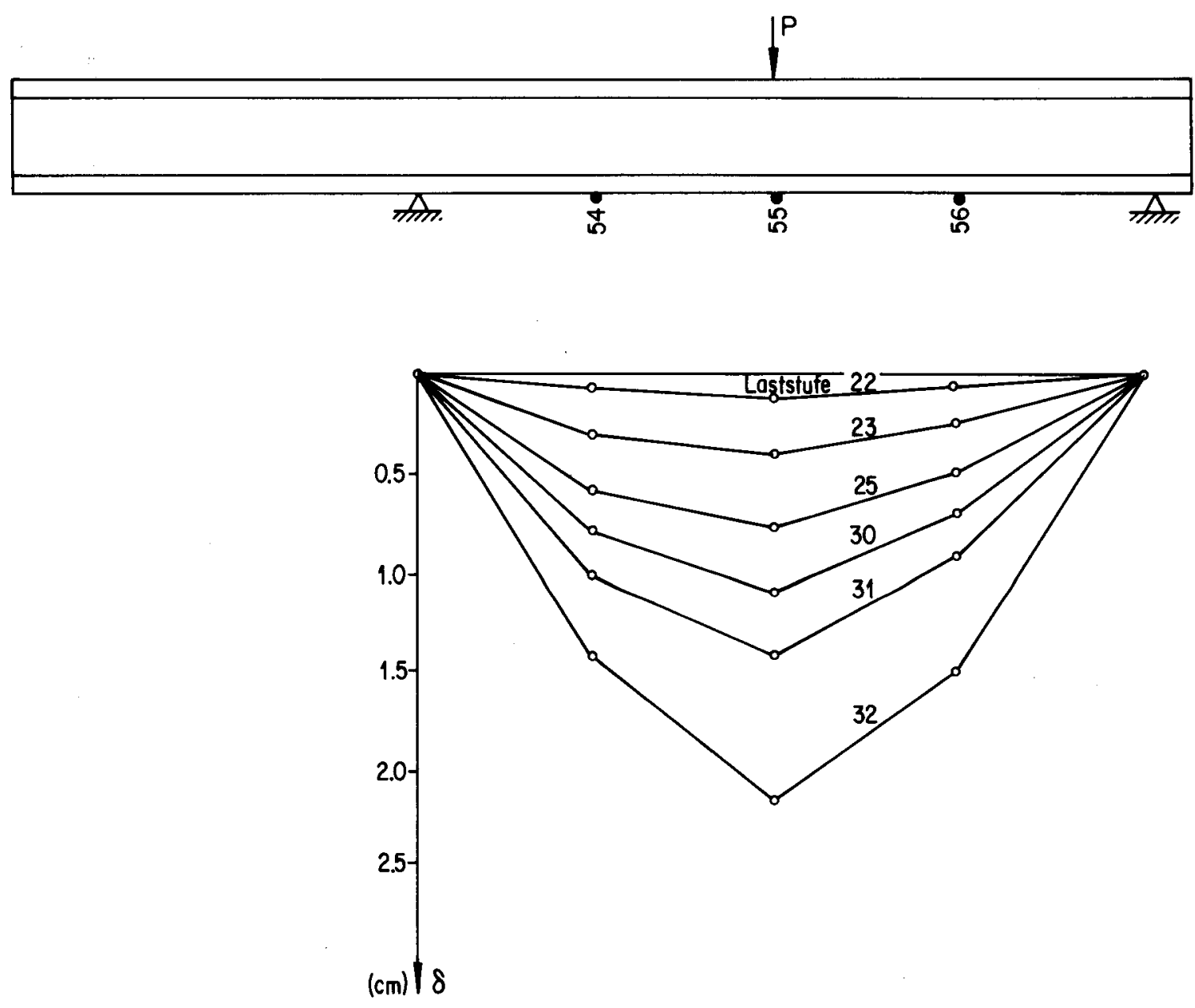

Bild 51: $\mathrm{S}_{2}$ Durchbiegung Laststellung II 

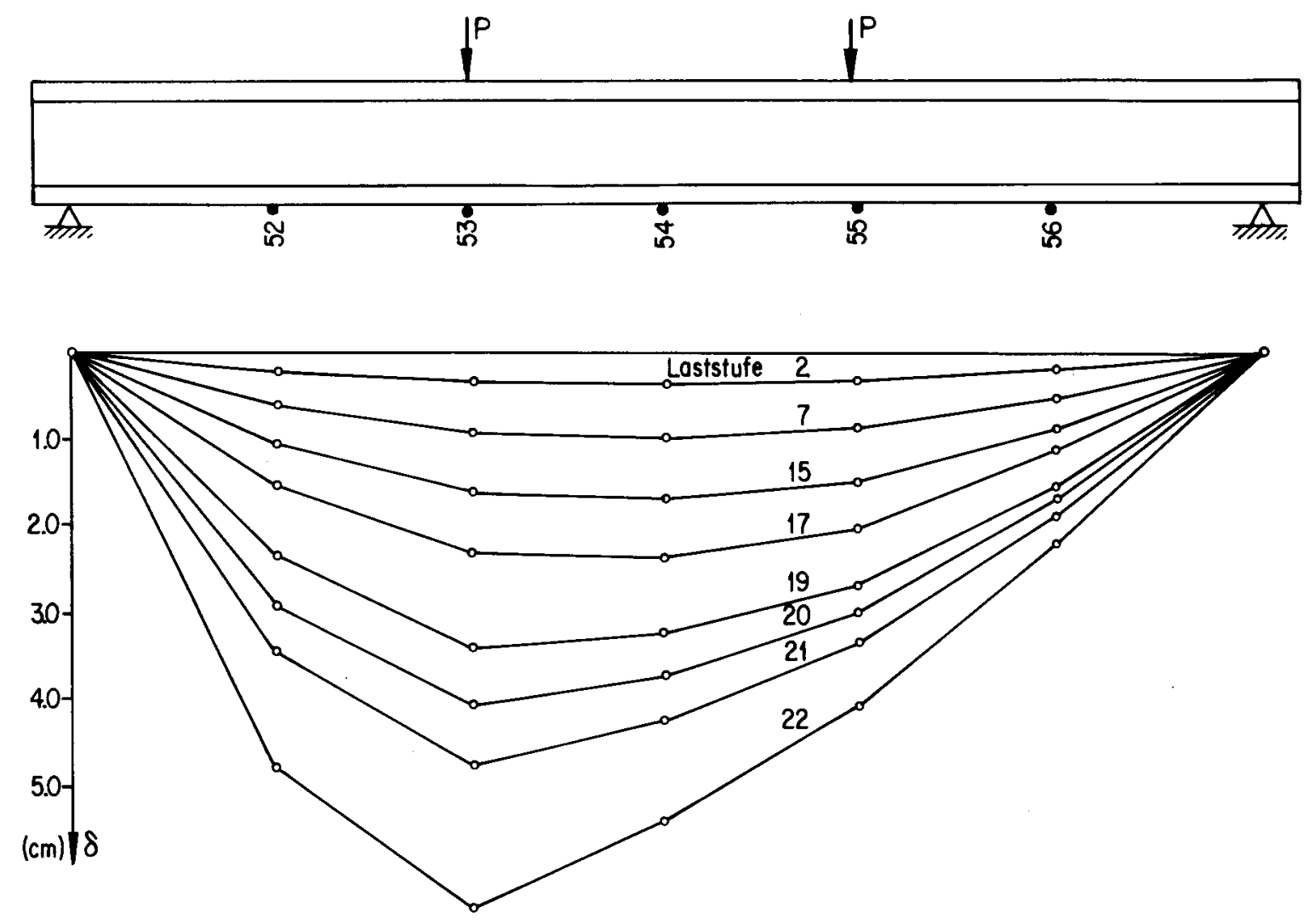

Bild 52: $S_{3}$ Durchbiegung Laststellung I
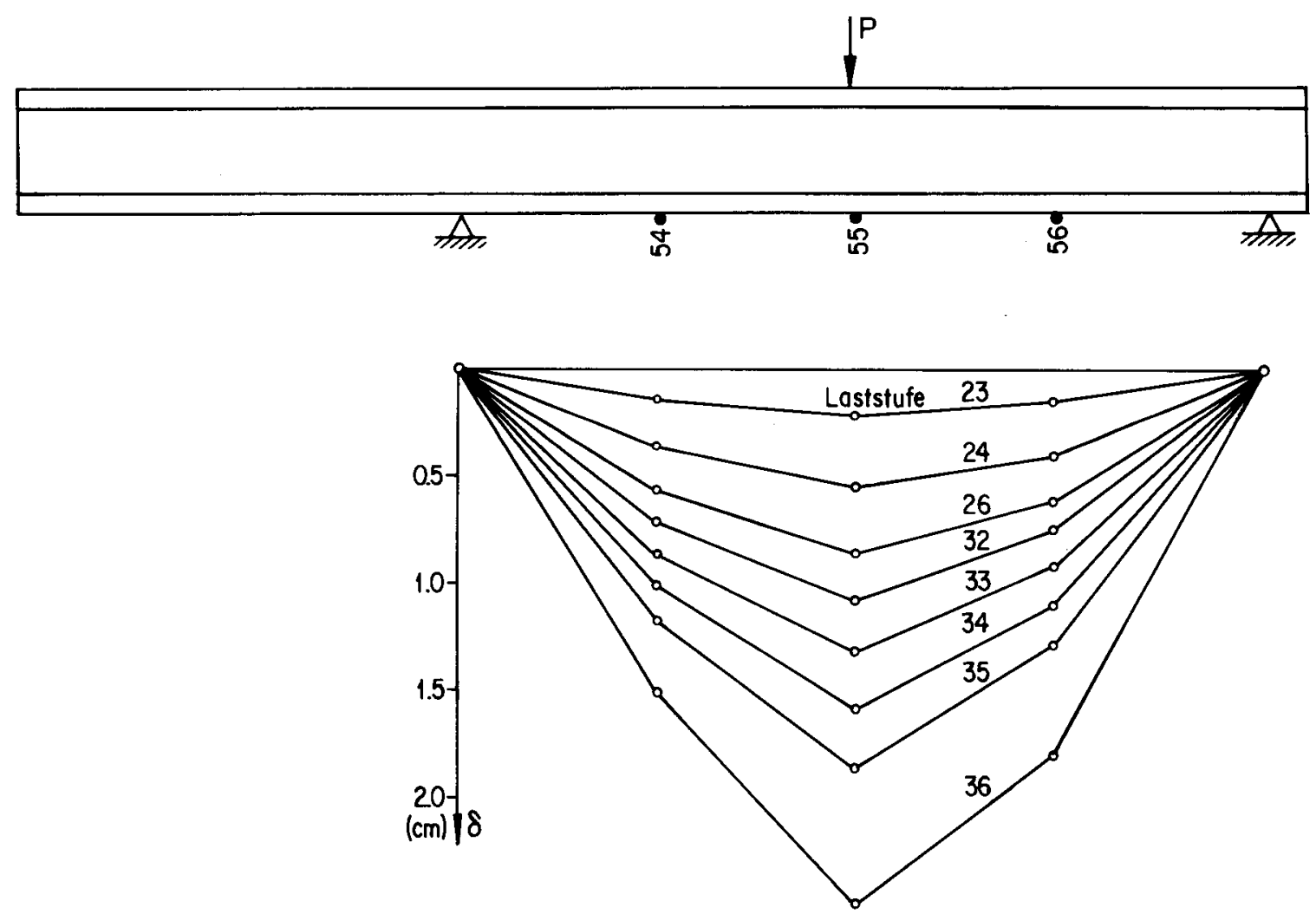

Bild 53: $\mathrm{S}_{3}$ Durchbiegung Laststellung II 

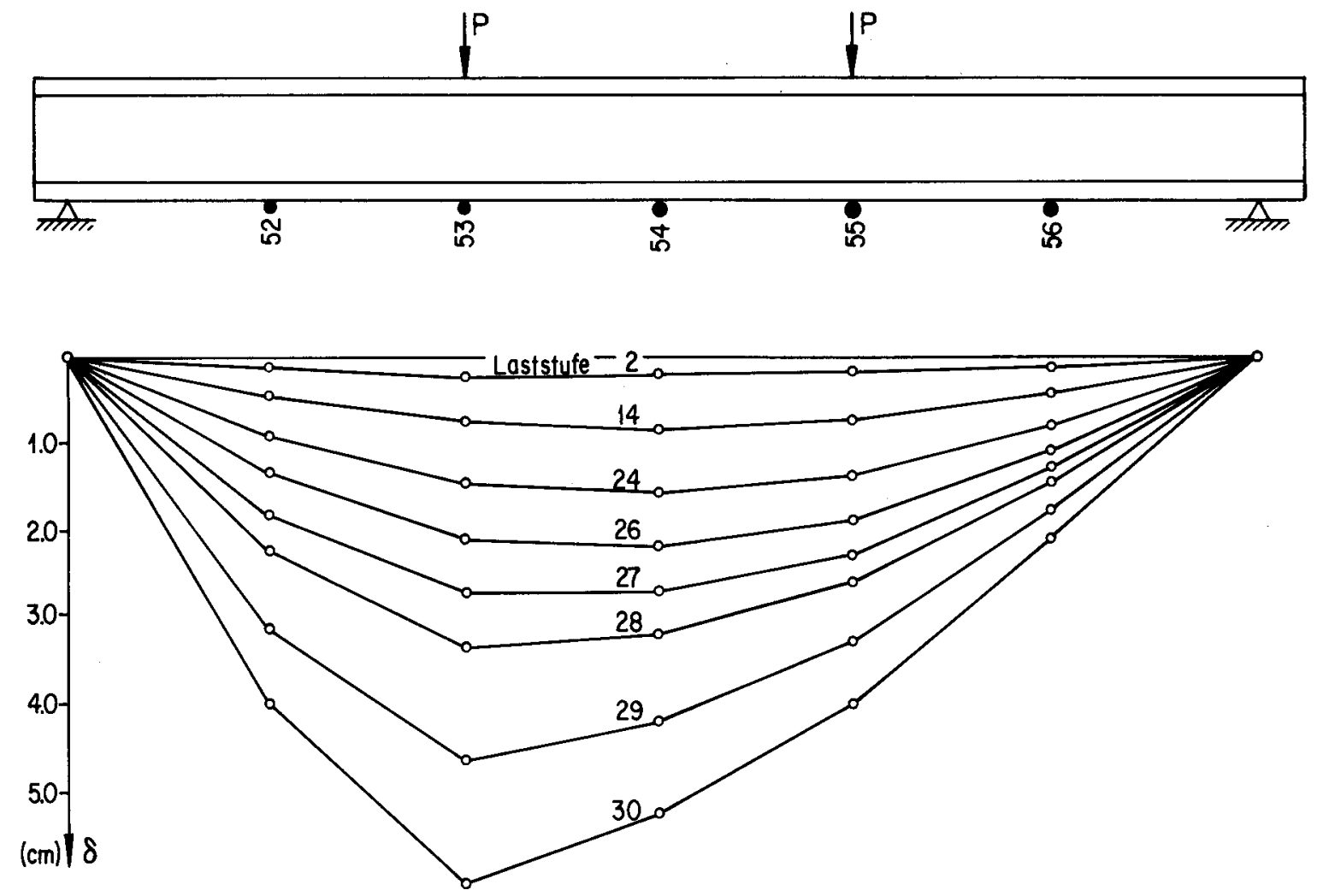

Bild 54: $\mathrm{S}_{4}$ Durchbiegung Laststellung I
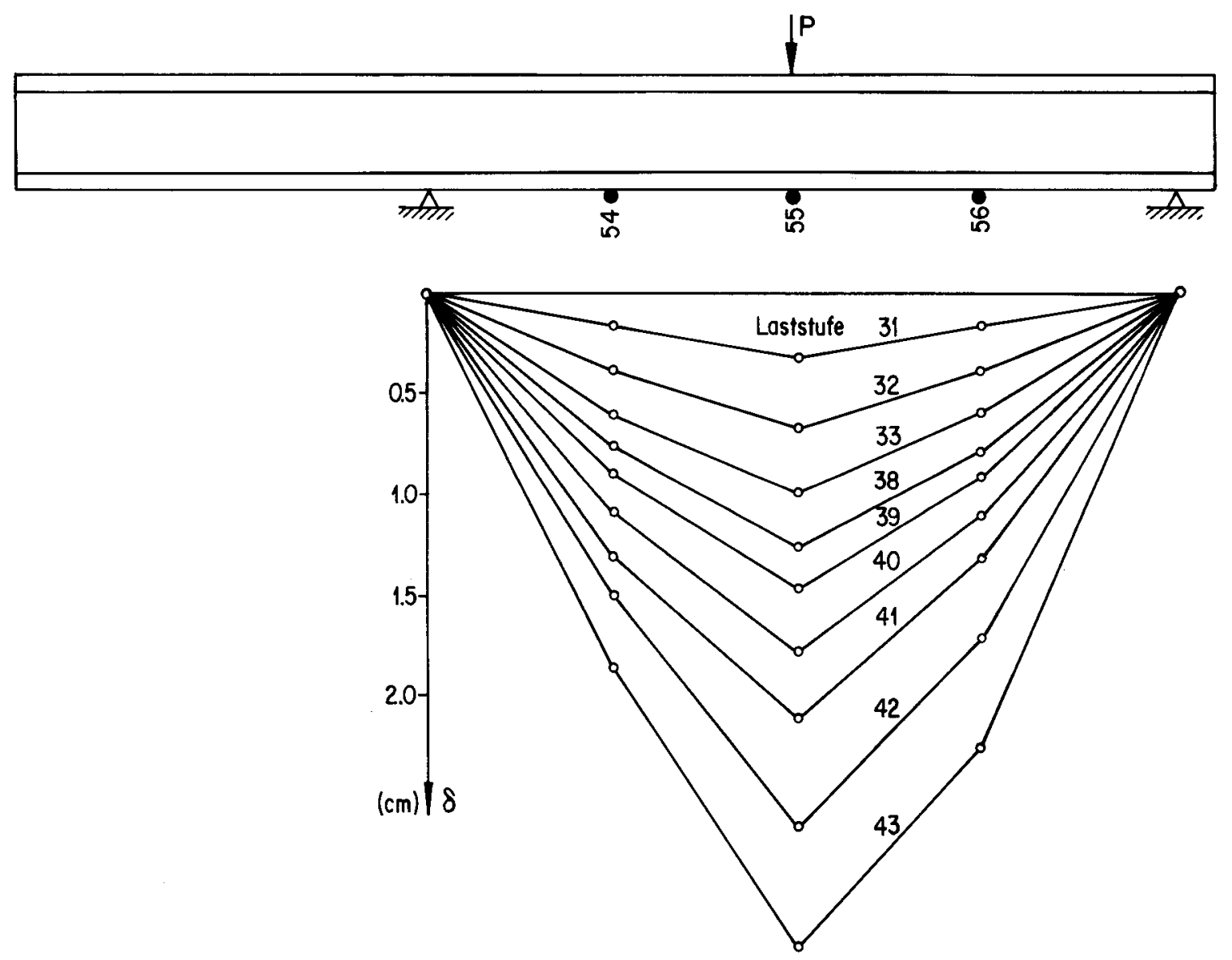

Bild 55: $\mathrm{S}_{4}$ Durchbiegung Laststellung II 

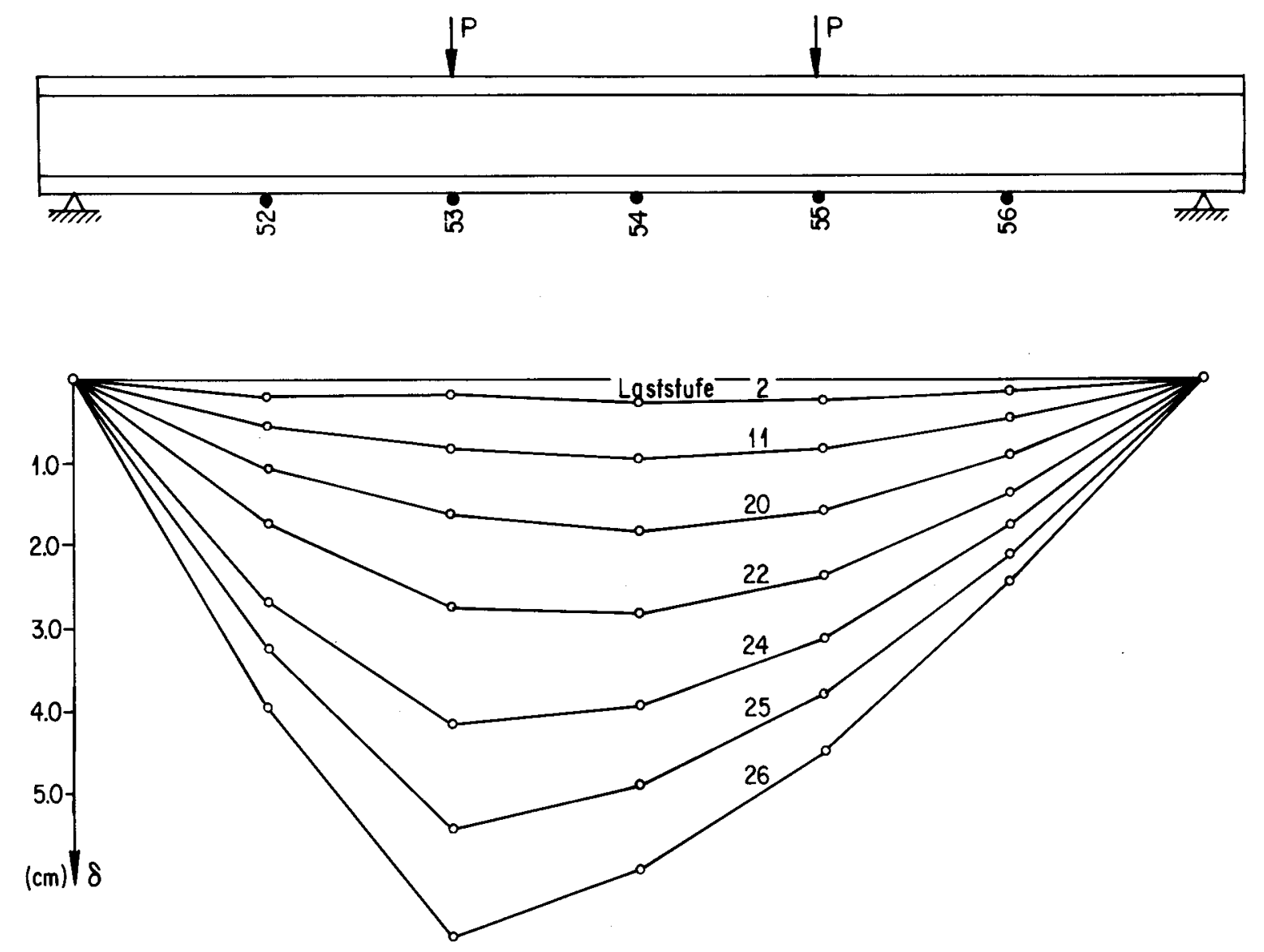

Bild 56: $\mathrm{S}_{5}$ Durchbiegung Laststellung I
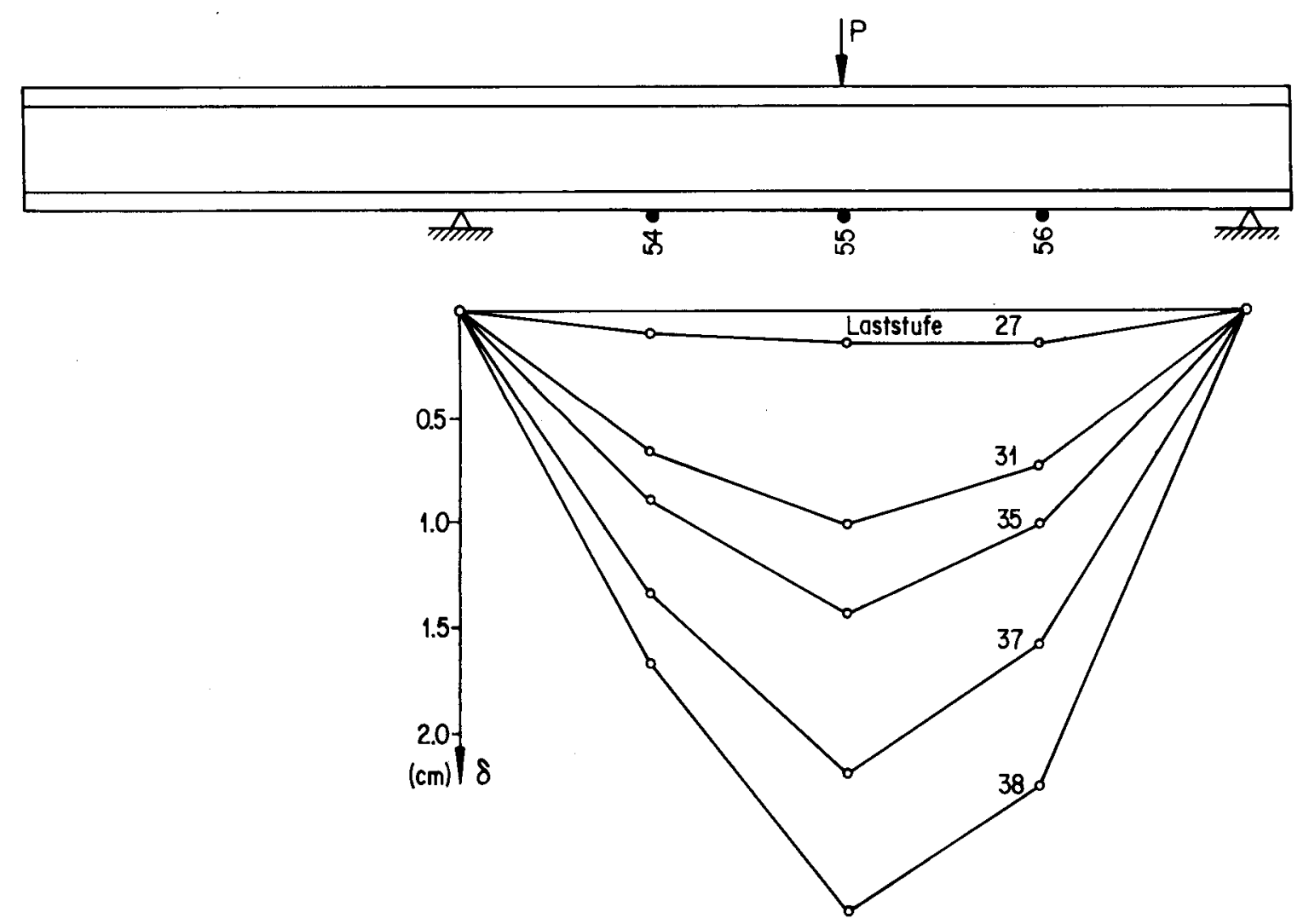

Bild 57: $\quad S_{5}$ Durchbiegung Laststellung II 
Loststellung I

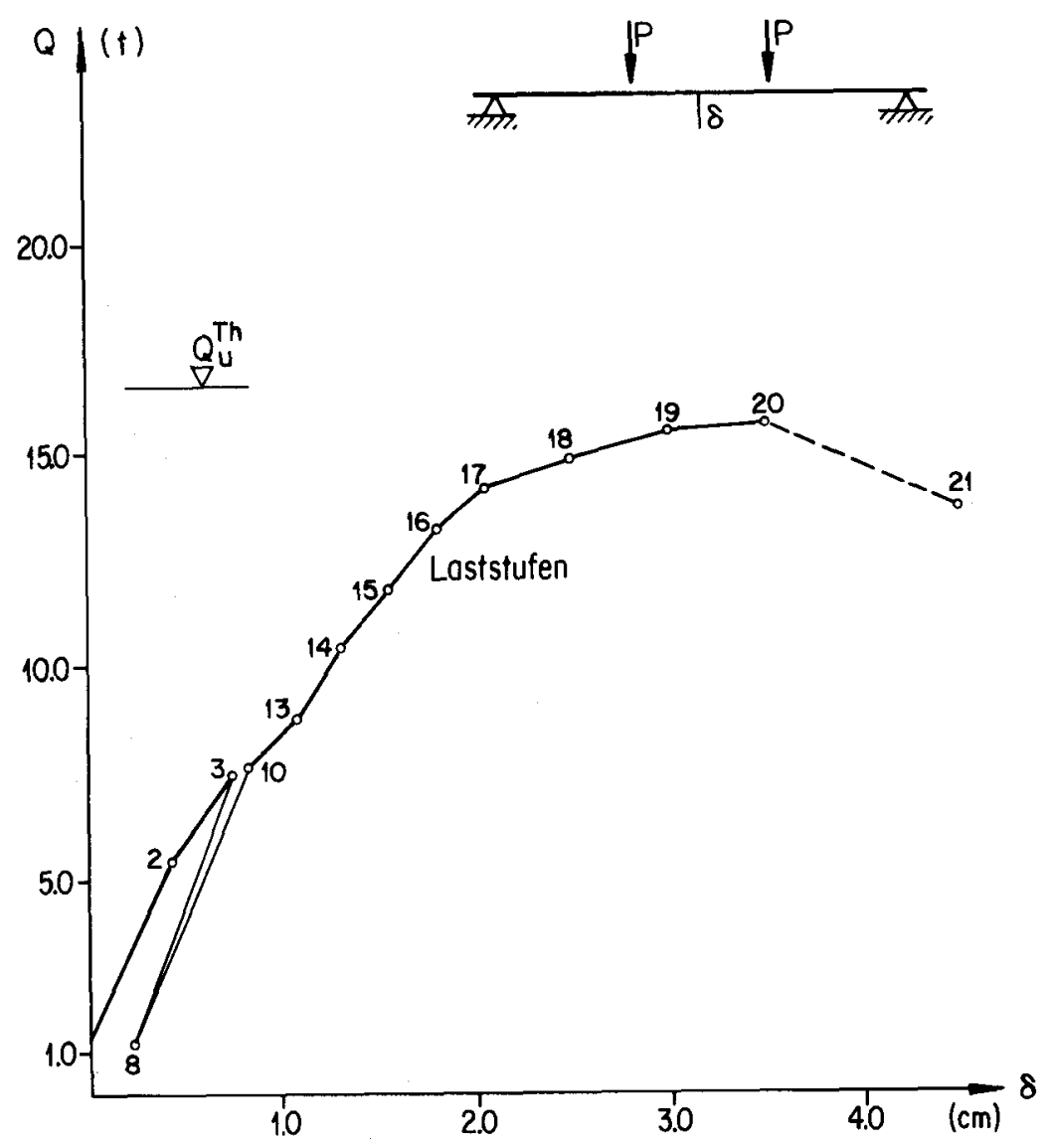

Bild 58: $\mathrm{S}_{6}$ Durchbiegung in Balkenmitte
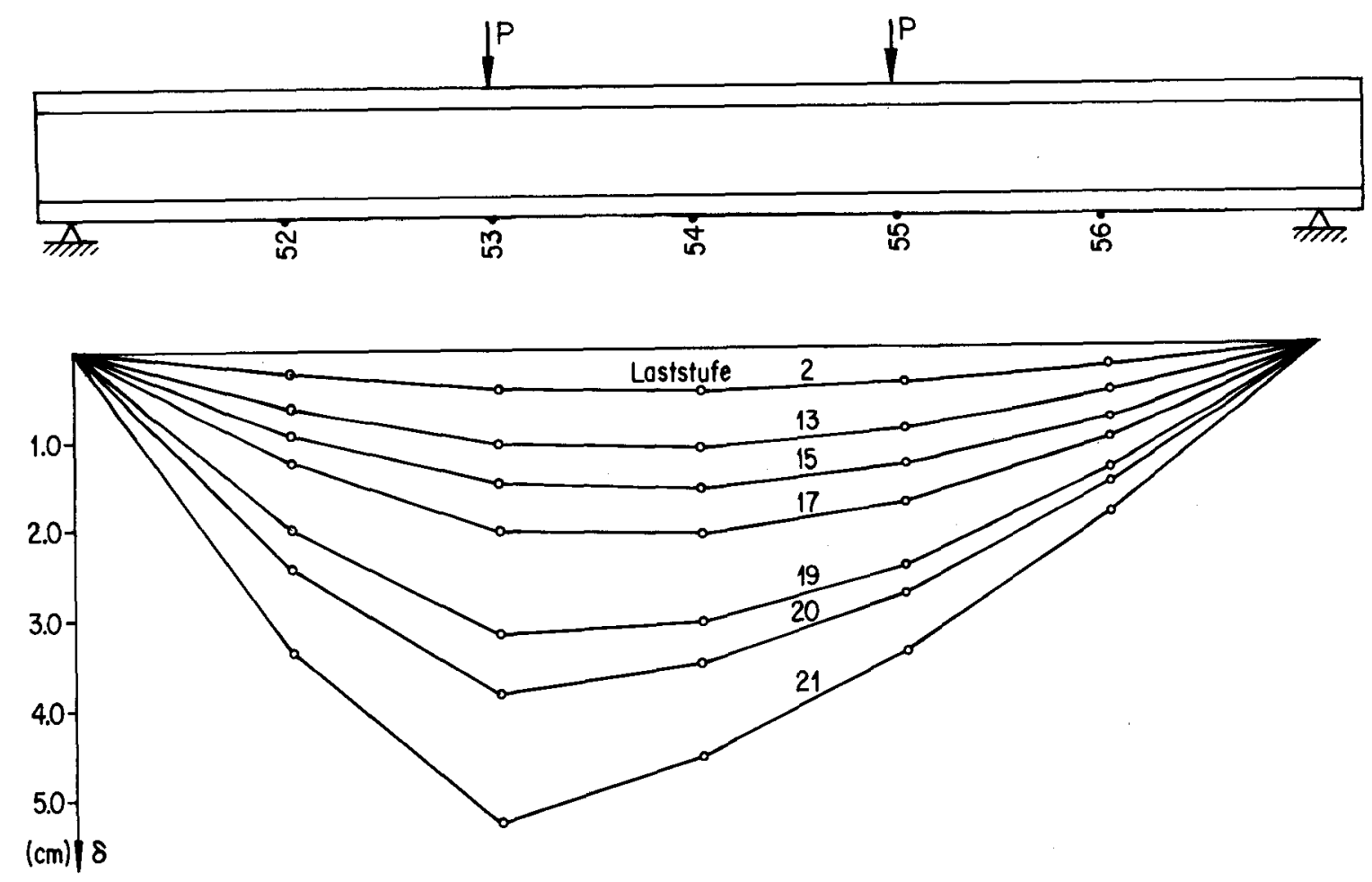

Bild 59: $S_{6}$ Durchbiegung Laststellung I 


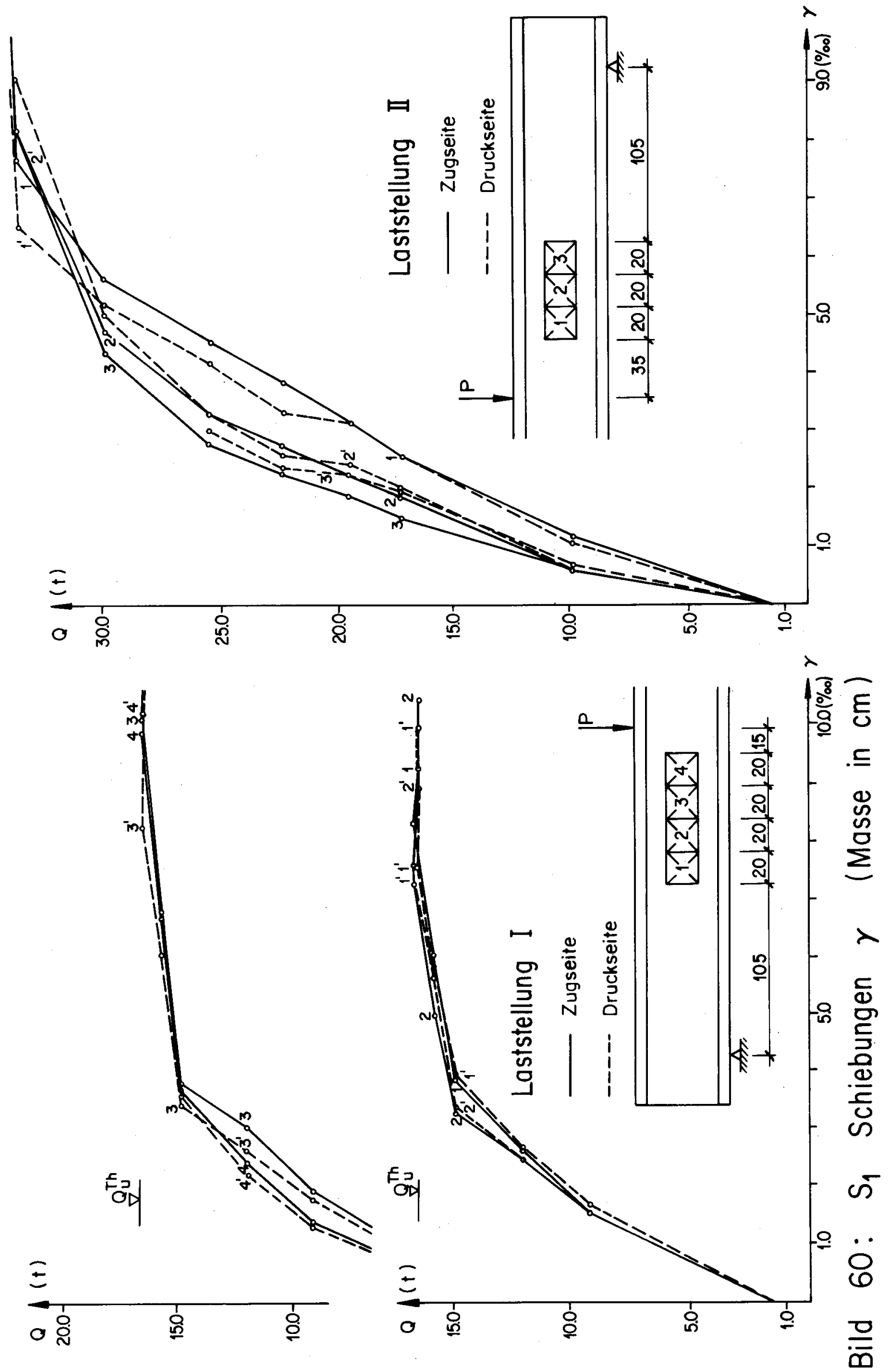




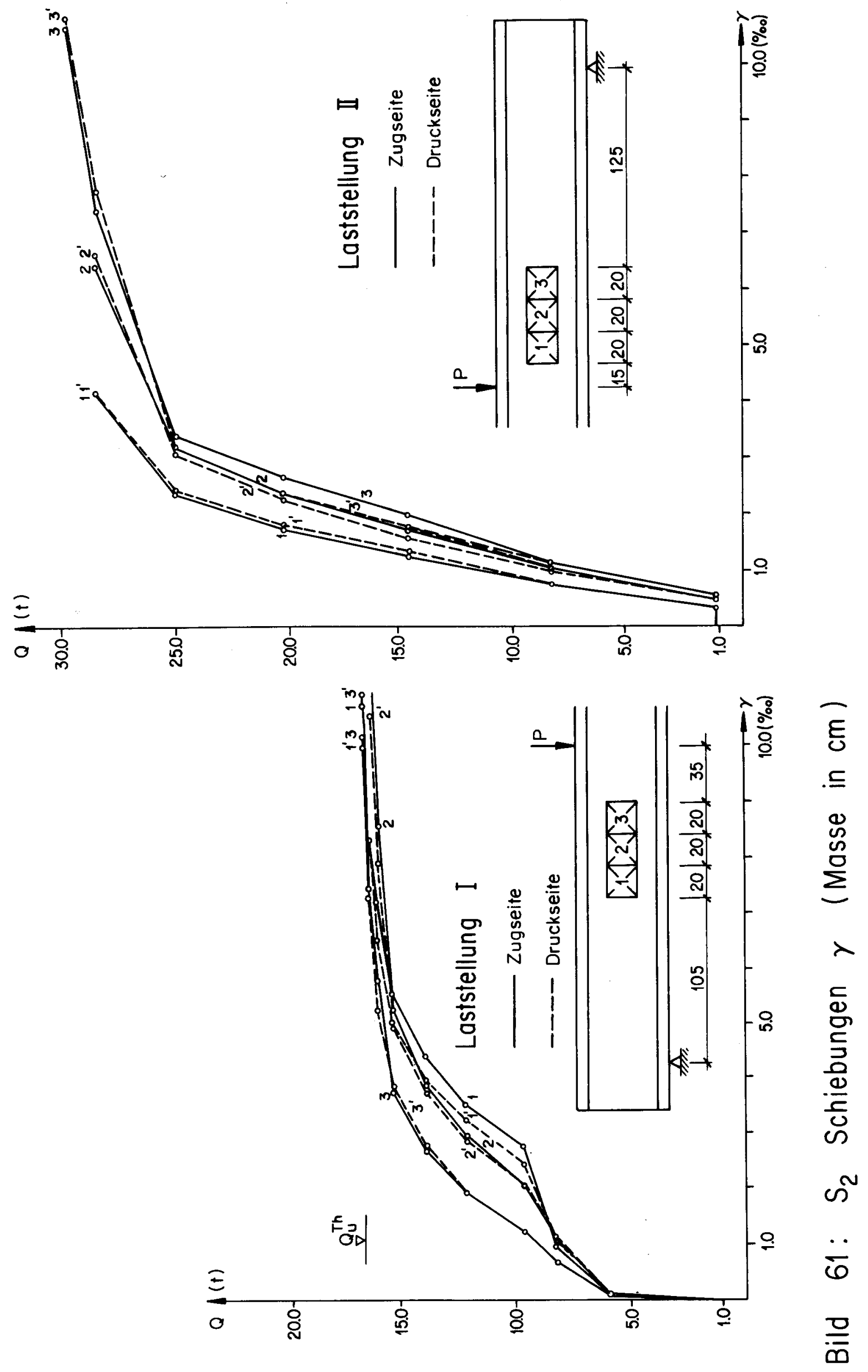



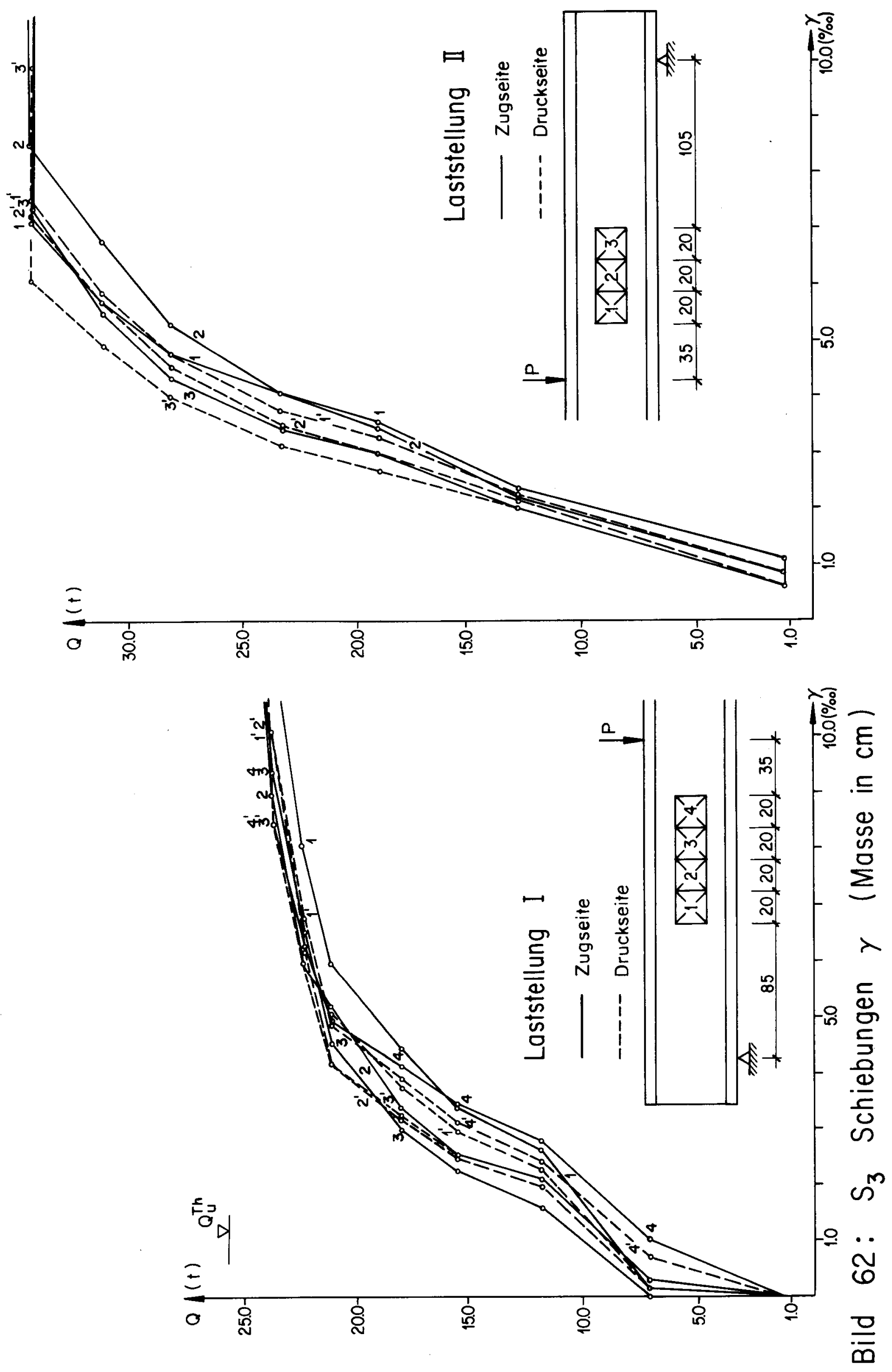


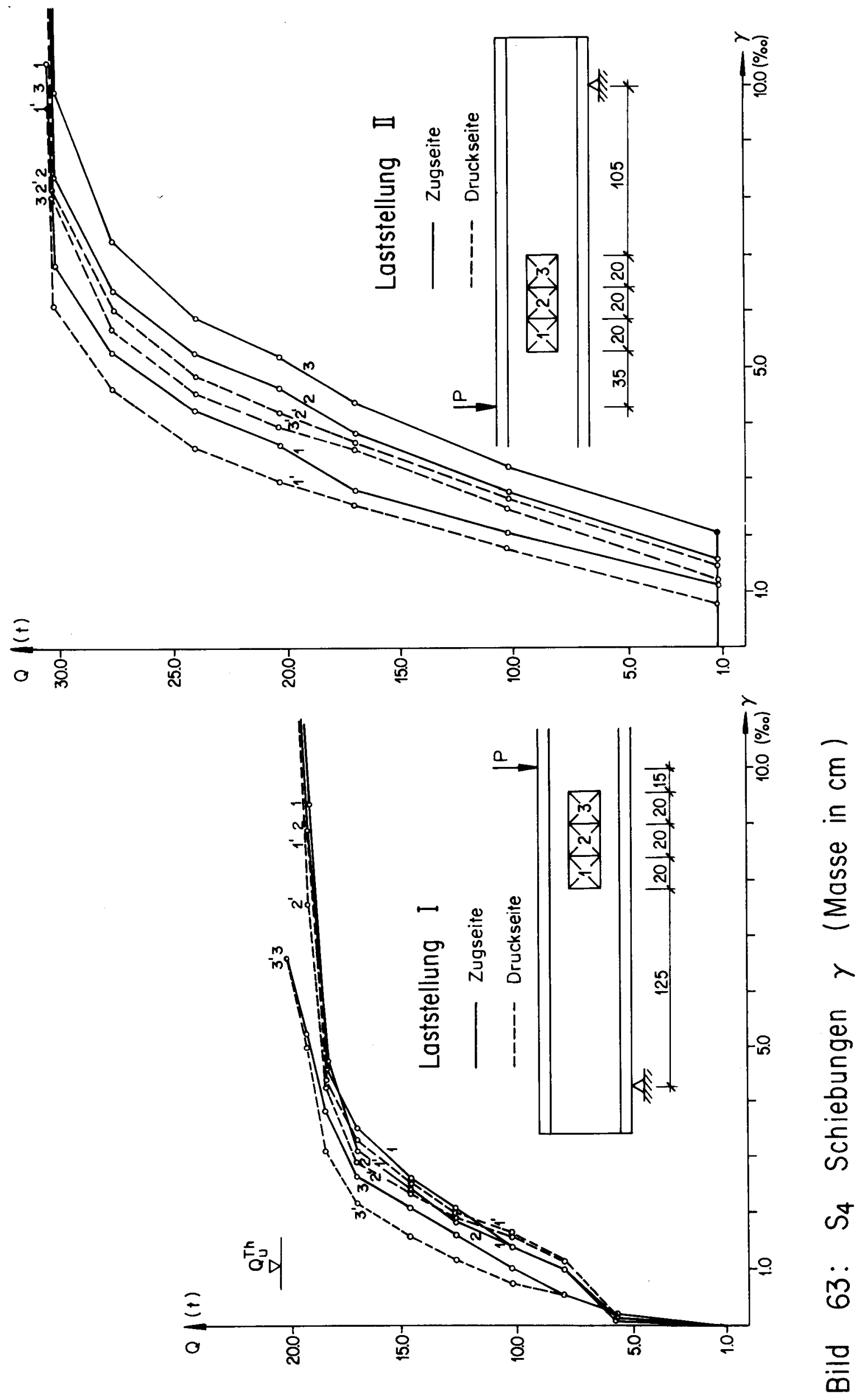




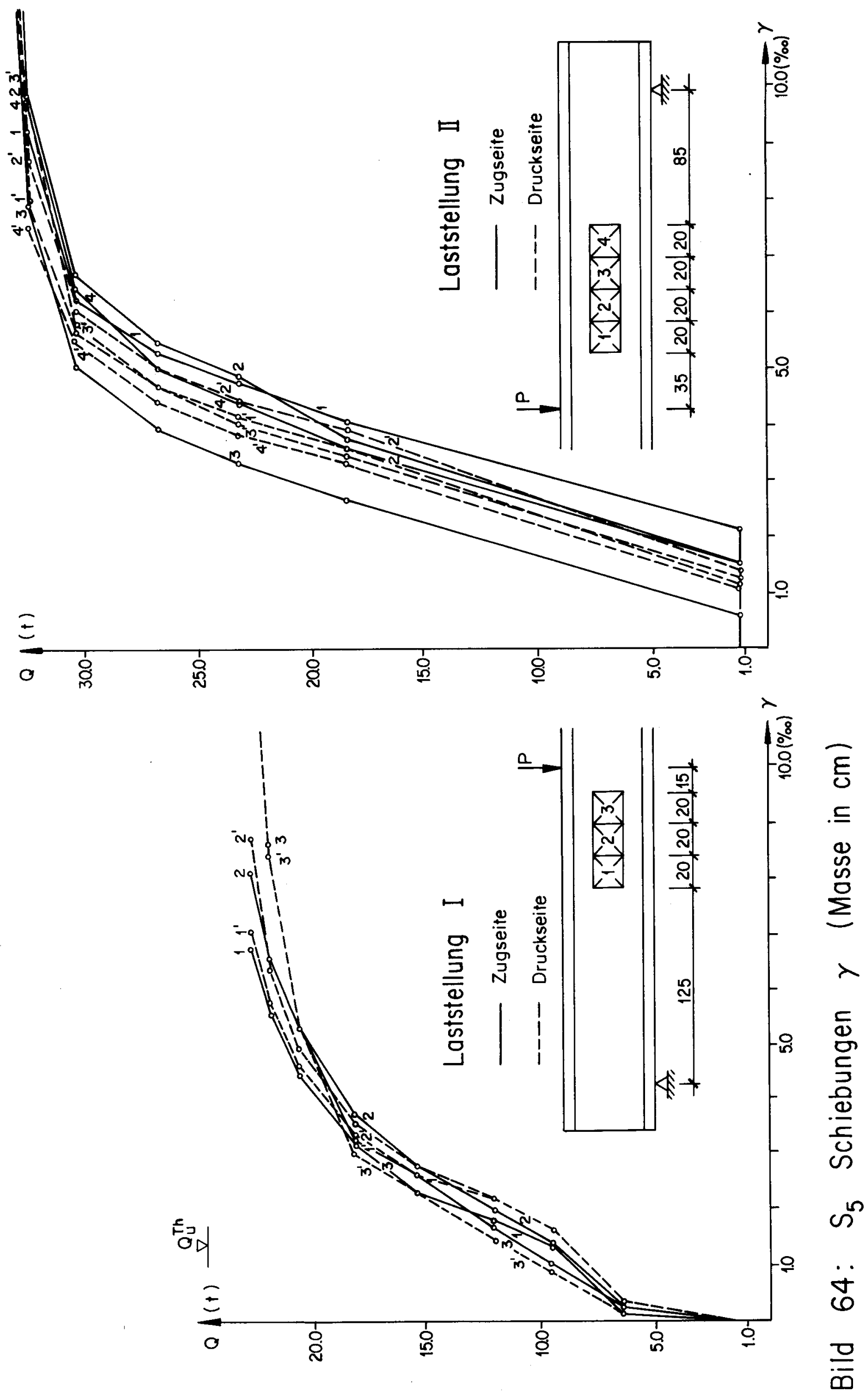




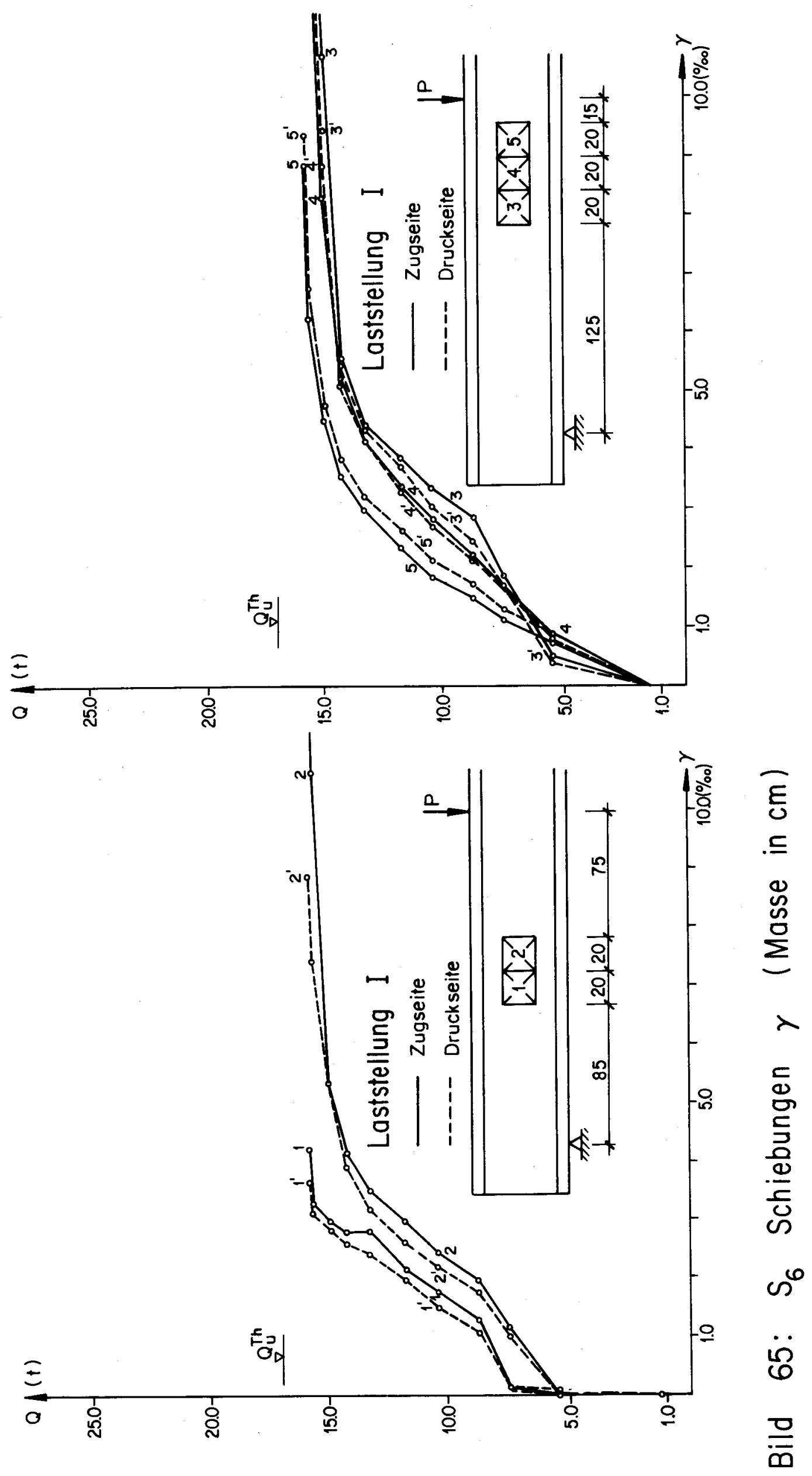



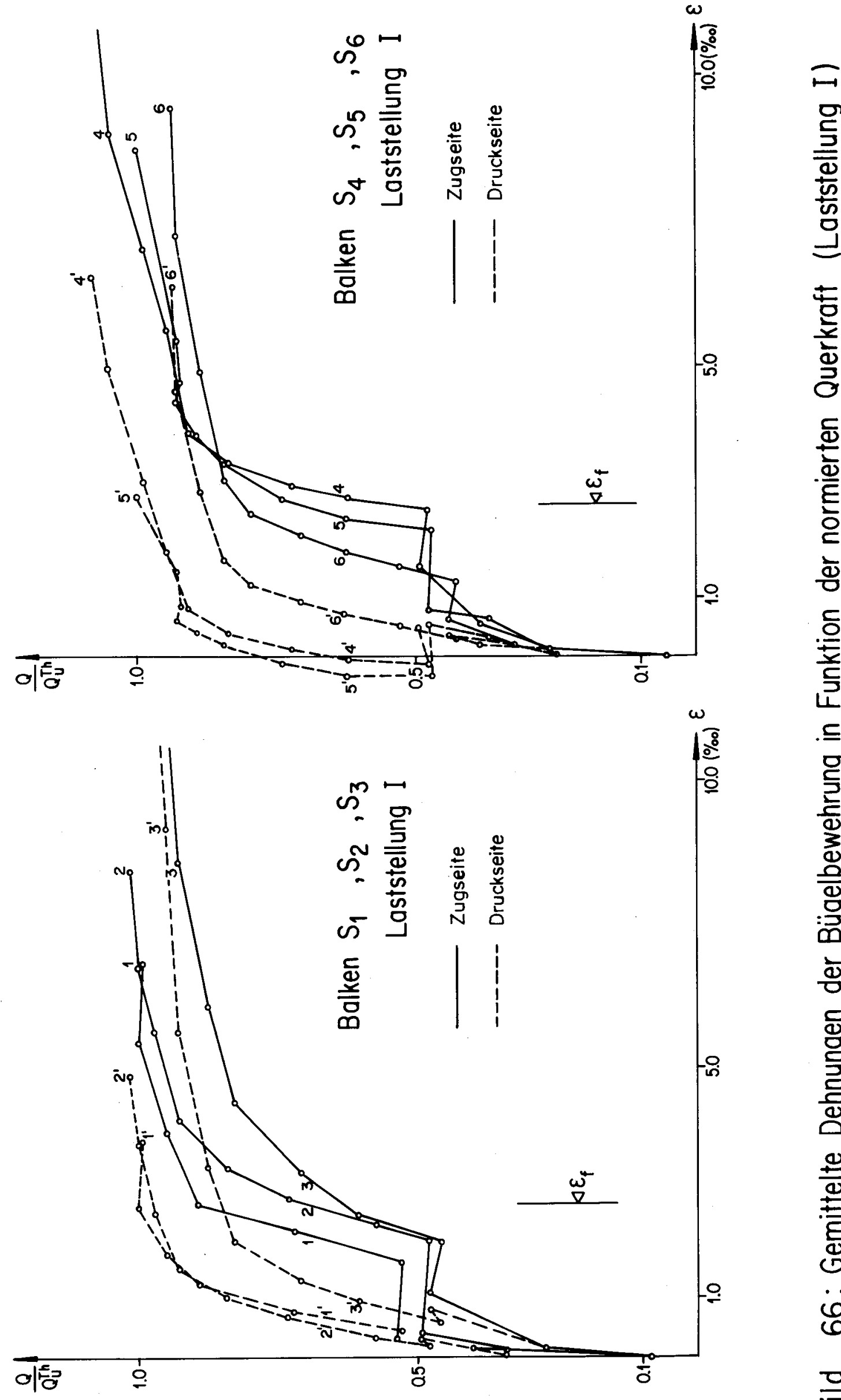

.

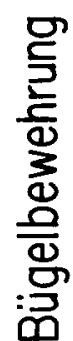

ষ্

这

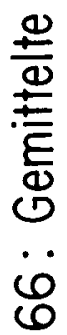

흠 


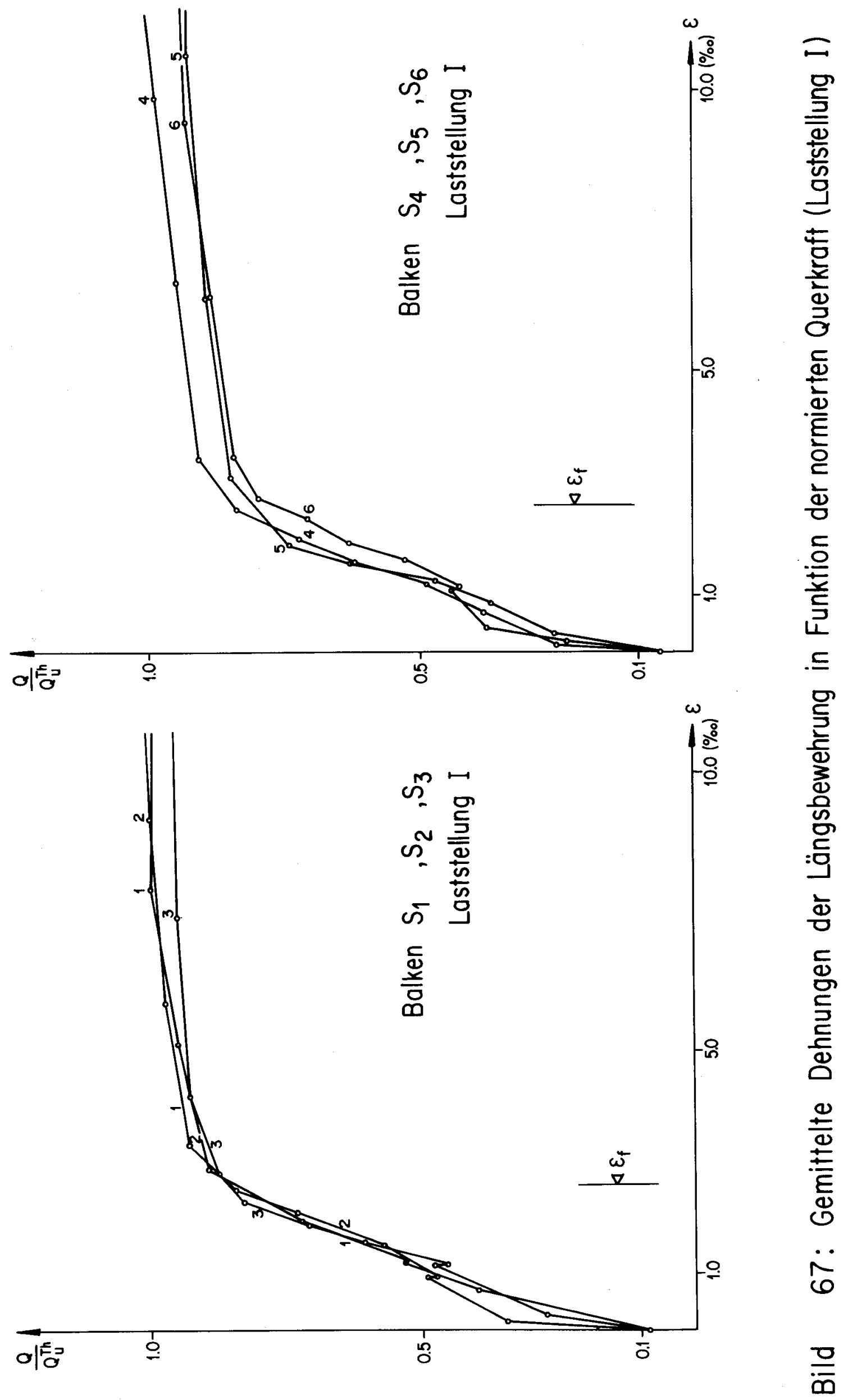




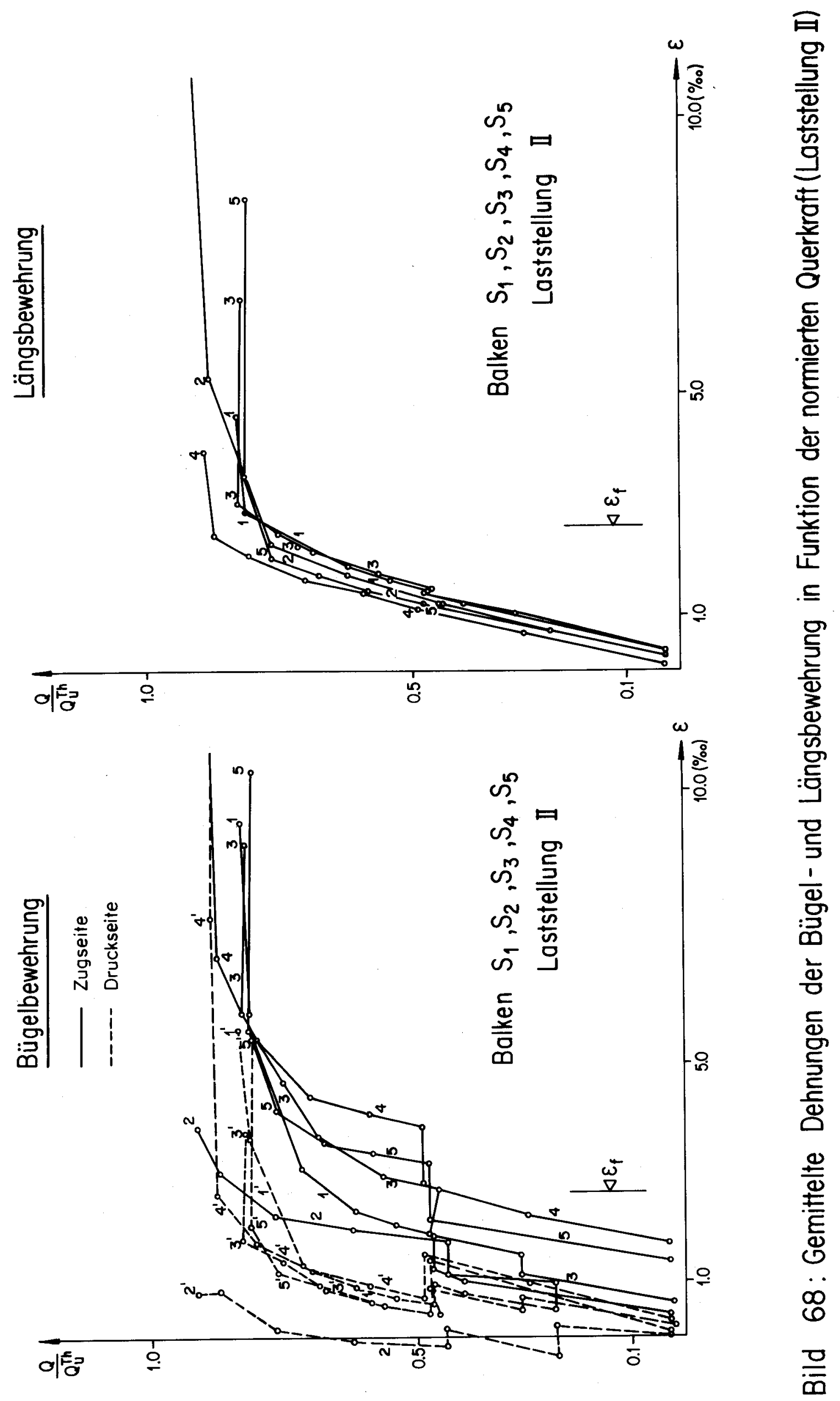




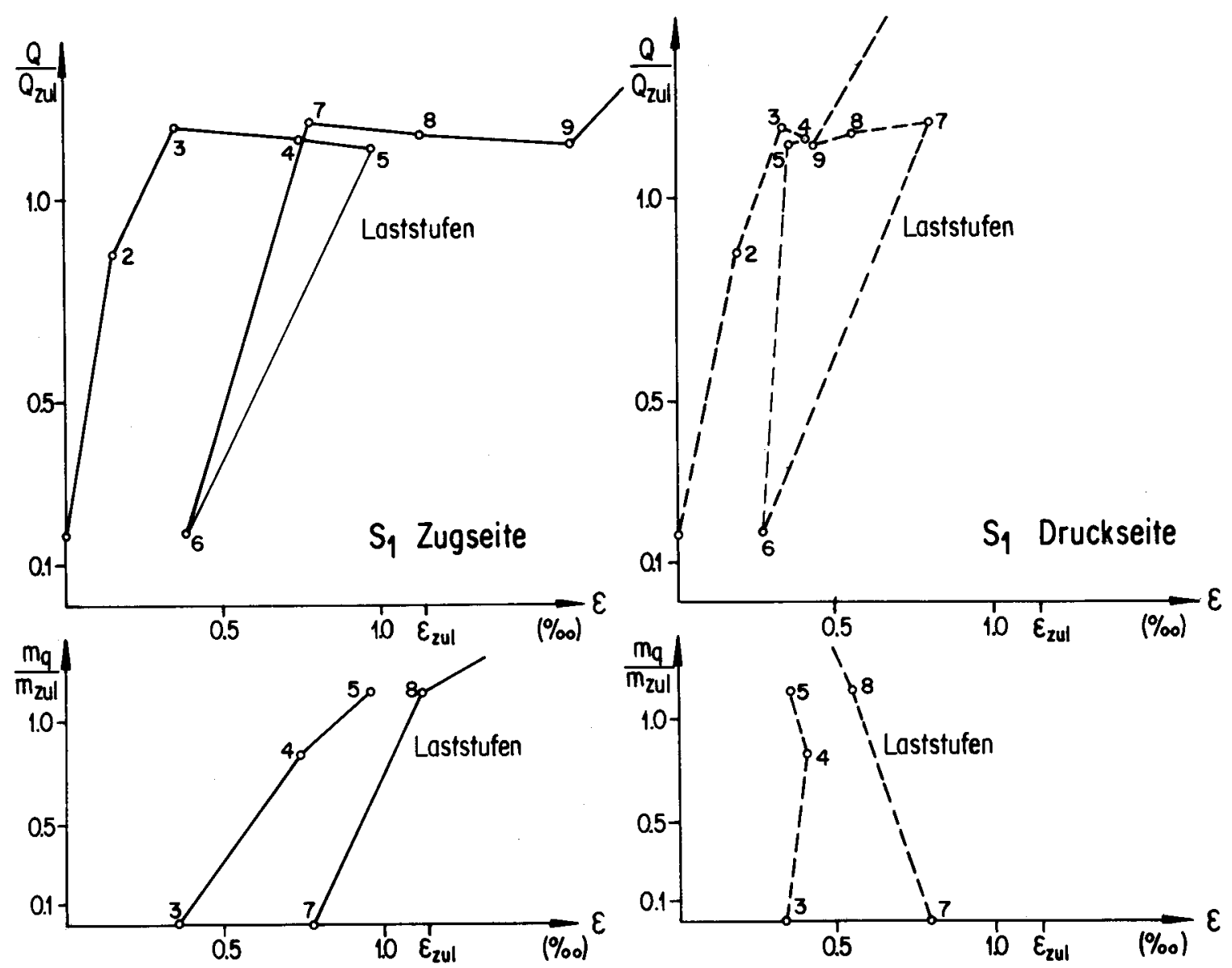

Bild 69: S1 LS I Gemittelte Bügeldehnungen bei kombinierter Belastung
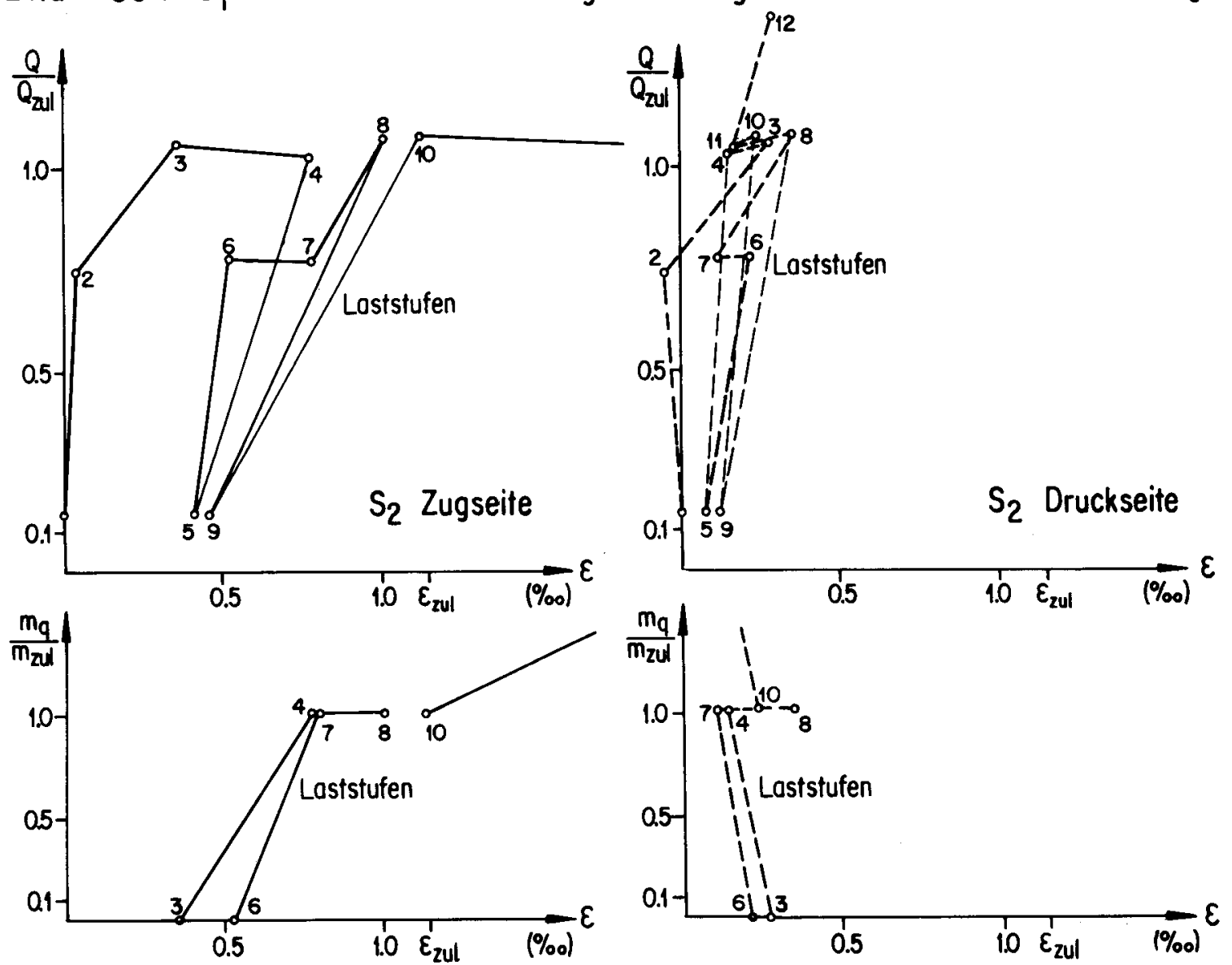

Bild 70: $S_{2}$ LS I Gemittelte Bügeldehnungen bei kombinierter Belastung 

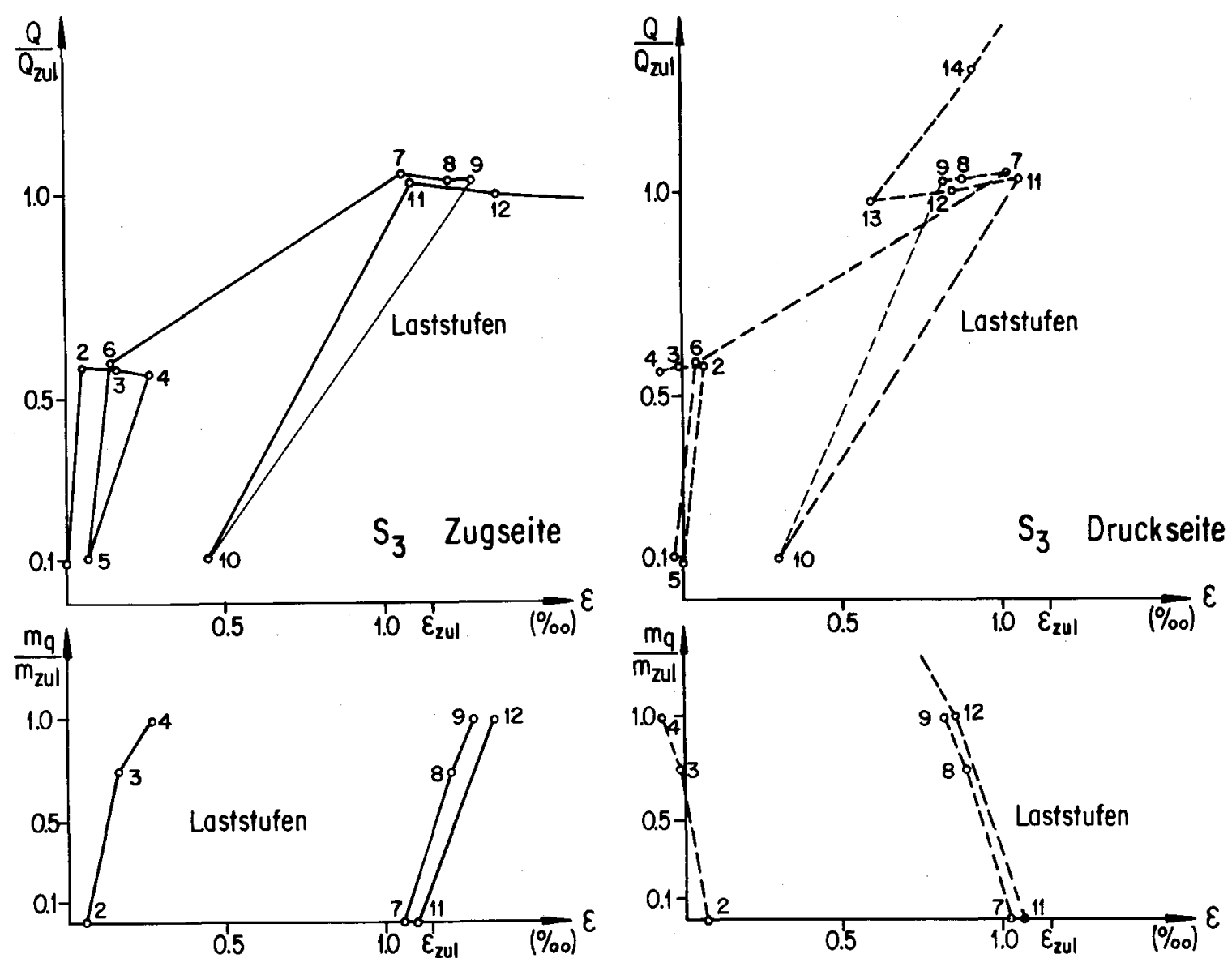

Bild 71: $S_{3}$ LSI Gemittelte Bügeldehnungen bei kombinierter Belastung
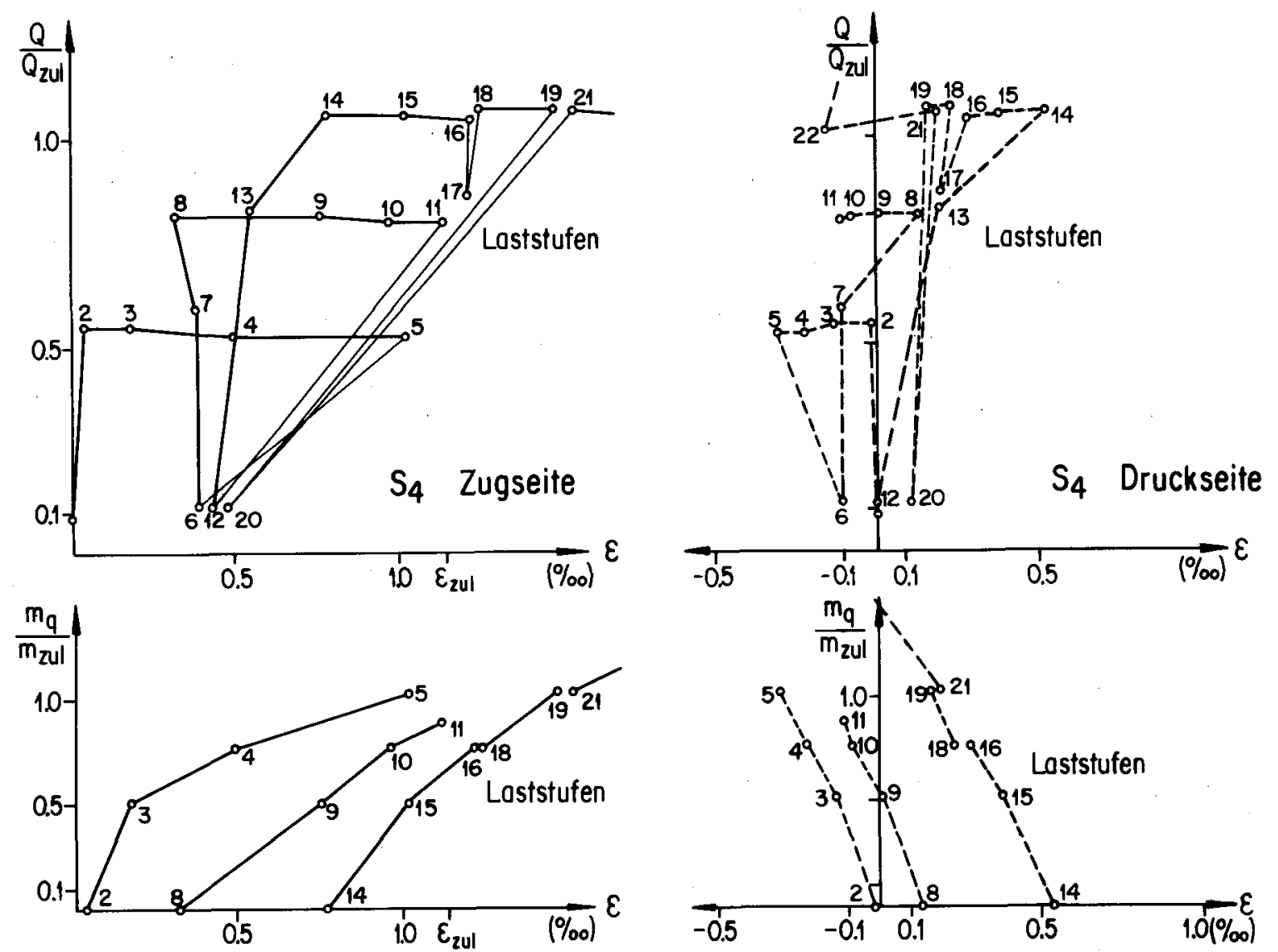

Bild 72: $\mathrm{S}_{4}$ LSI Gemittelte Bügeldehnungen bei kombinierter Belastung 


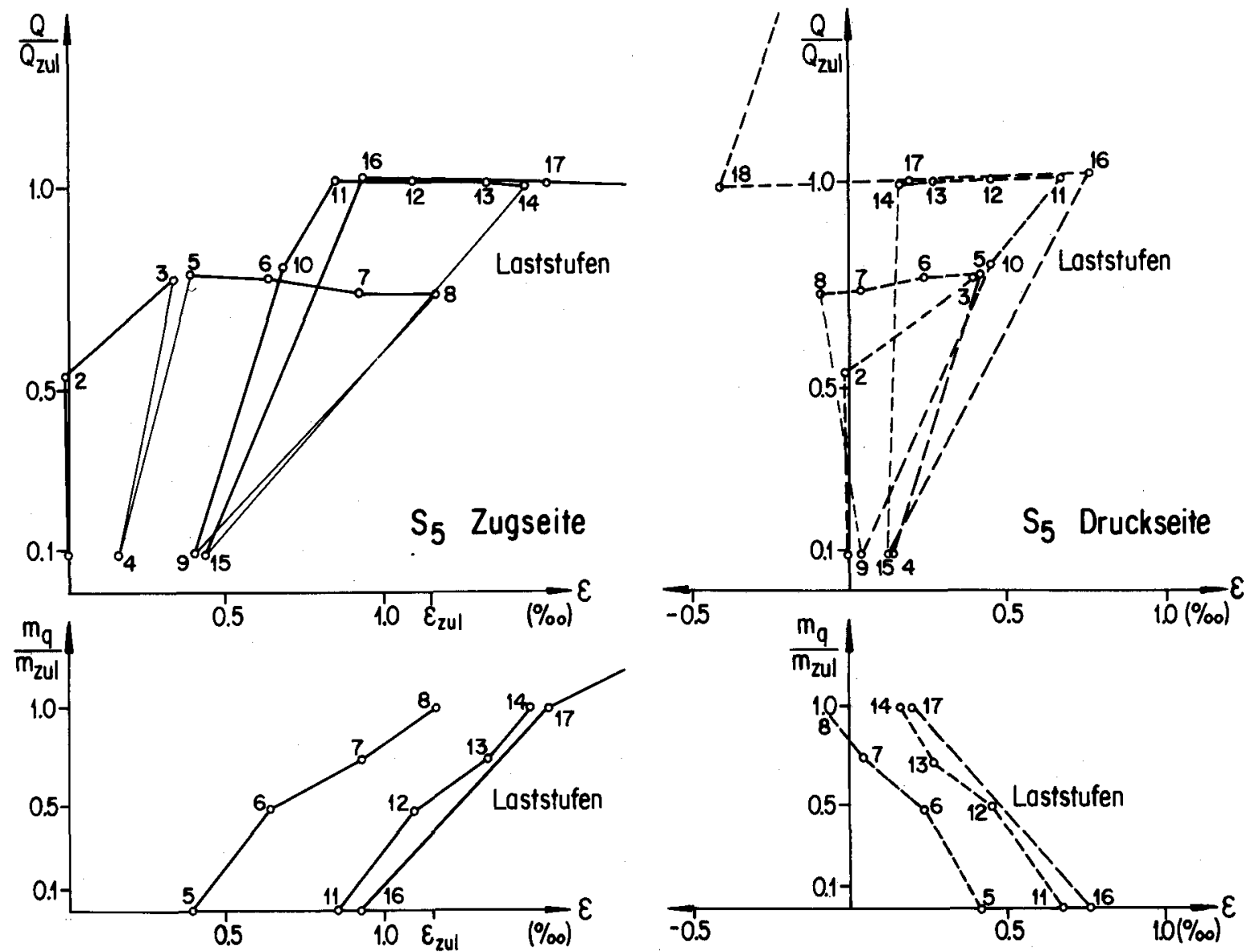

Bild 73: $S_{5}$ LS I Gemittelte Bügeldehnungen bei kombinierter Belastung
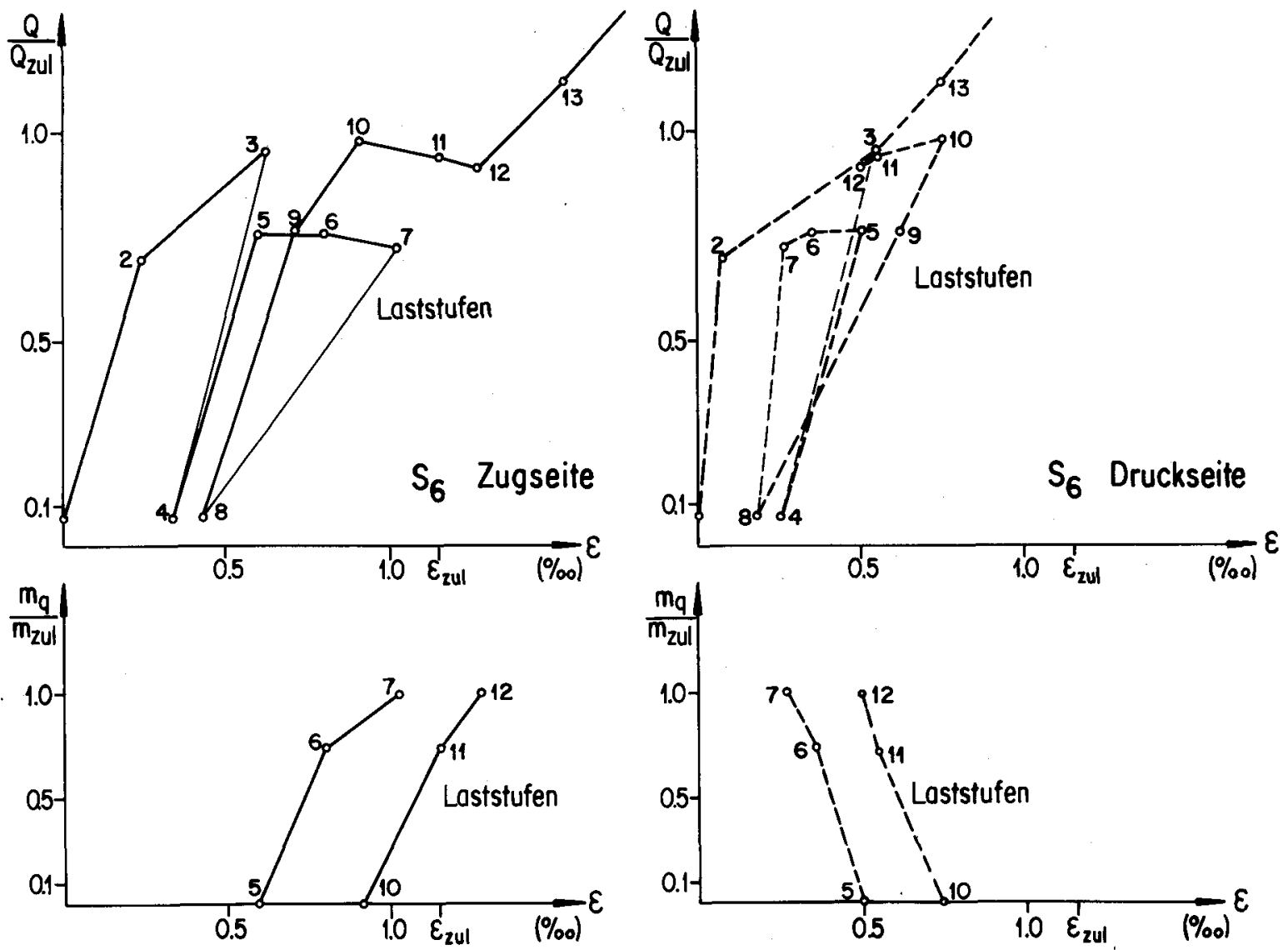

Bild 74: $S_{6}$ LS I Gemittelte Bügeldehnungen bei kombinierter Belastung 


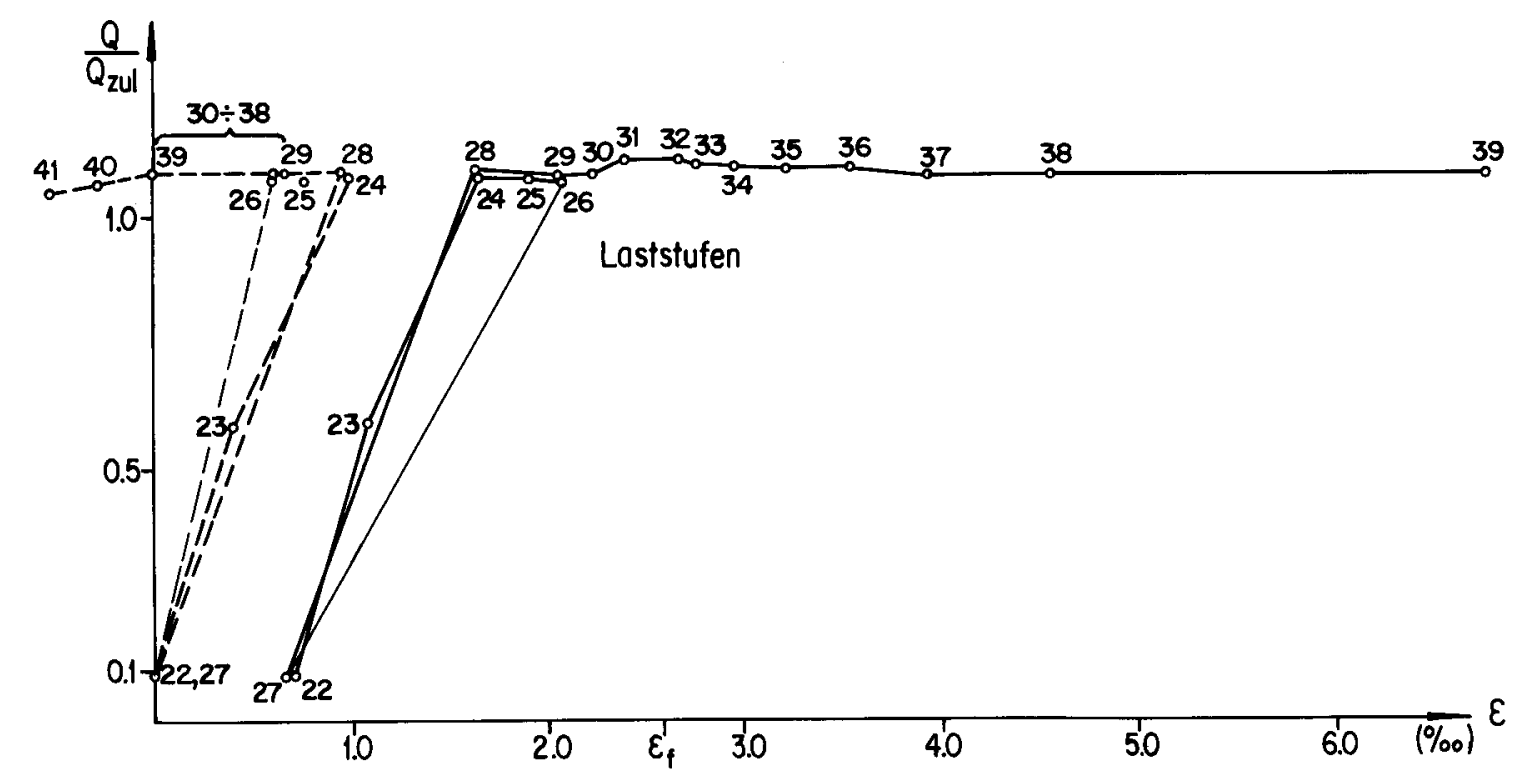

Bild 75: S6 LSII Gemittelte Bügeldehnungen in Funktion der Querkraft

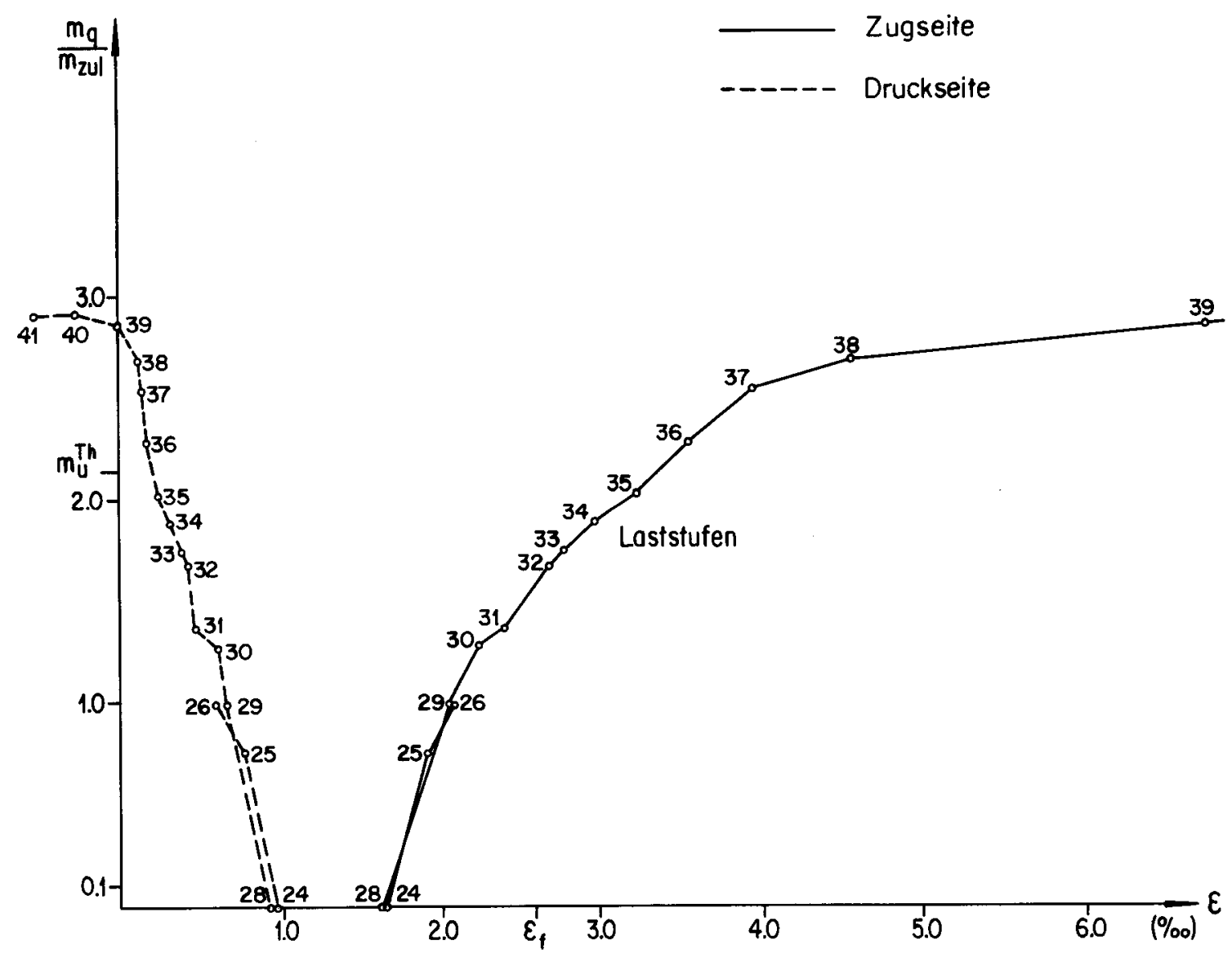

Bild 76: $S_{6}$ LSII Gemittelte Bügeldehnungen in Funktion der Querbiegung 


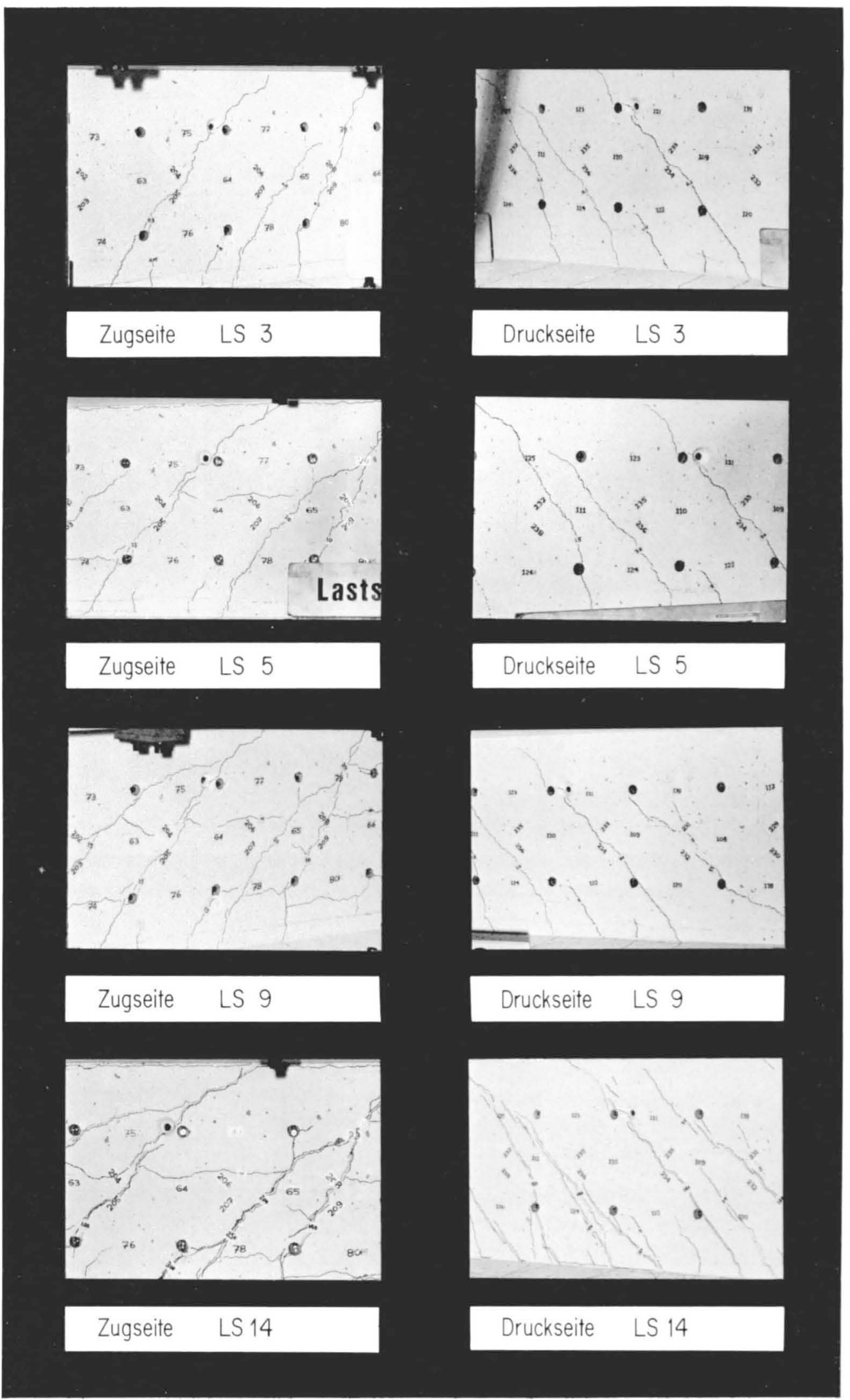

Zohlen bedeuten Messstellen sowie Rissweiten in 1/100 mm

Bild 77: Rissverlauf Balken $S_{1}$ Laststellung I 


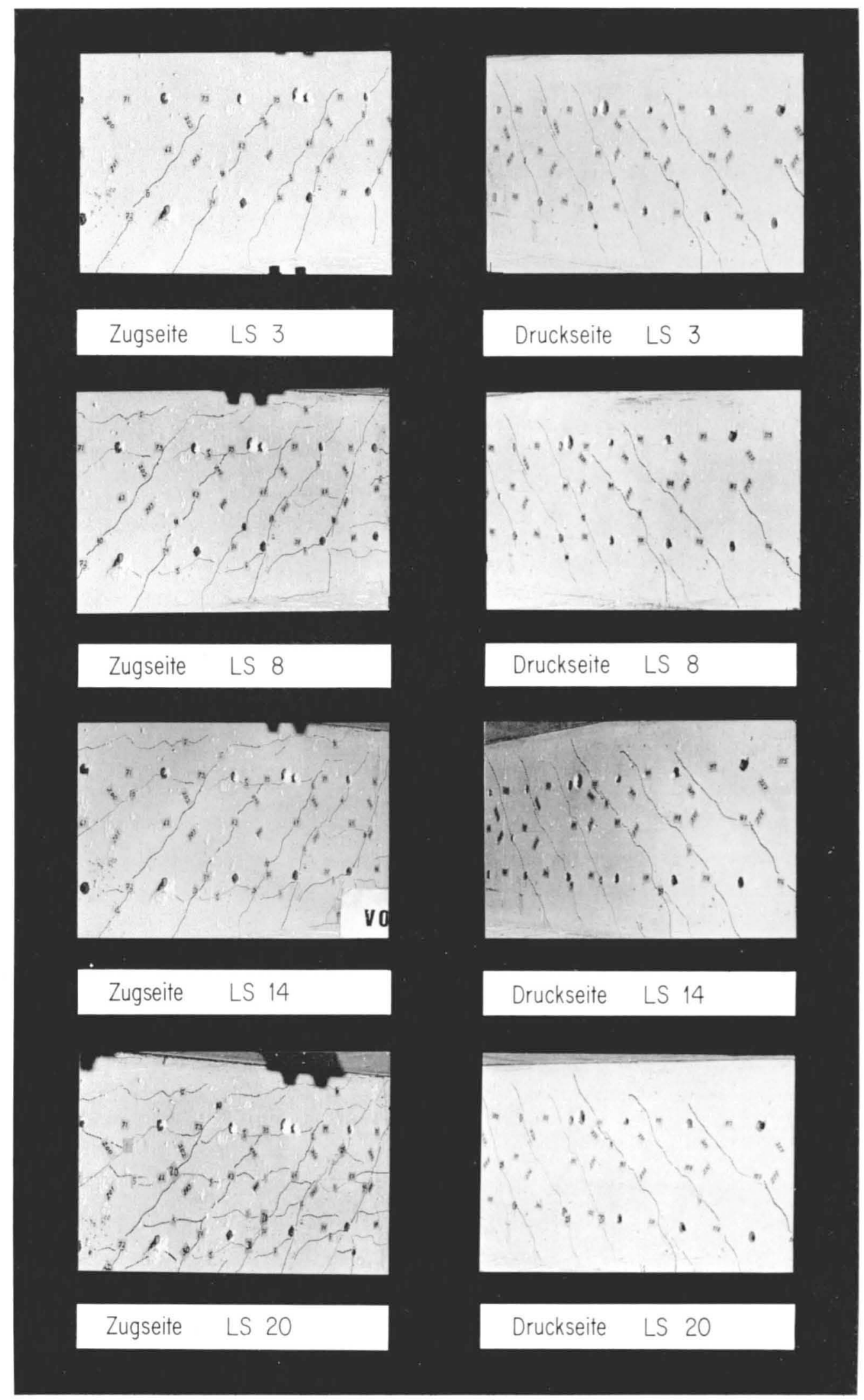

Zahlen bedeuten Messstellen sowie Rissbreiten in $1 / 100 \mathrm{~mm}$

Bild 78: Rissverlauf Balken $S_{5}$ Laststellung I 
- Loststufen

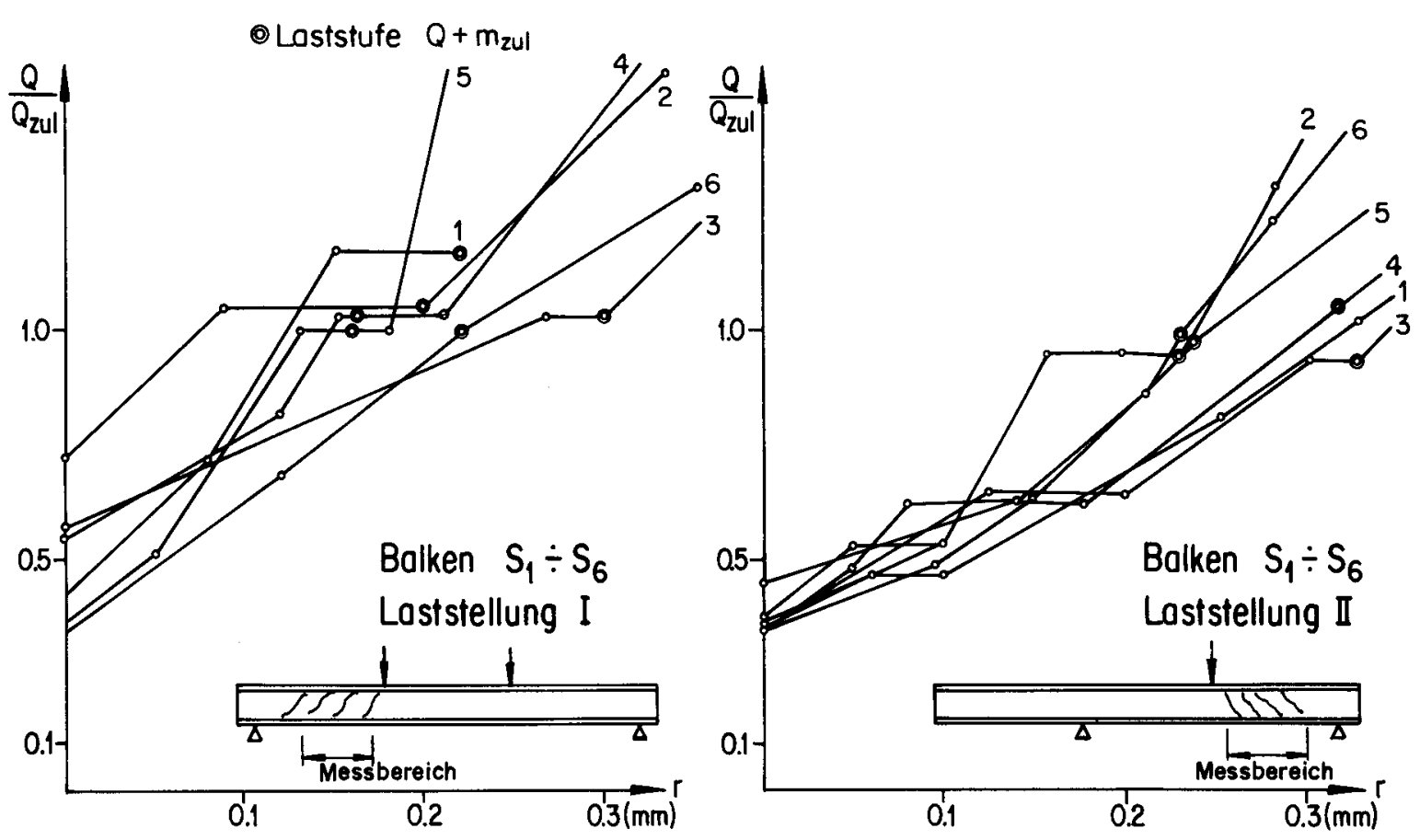

Bild 79: Gemittelte Rissbreiten bei kombinierter Belastung

- Laststufe

- Loststufe $Q+m_{z u l}$
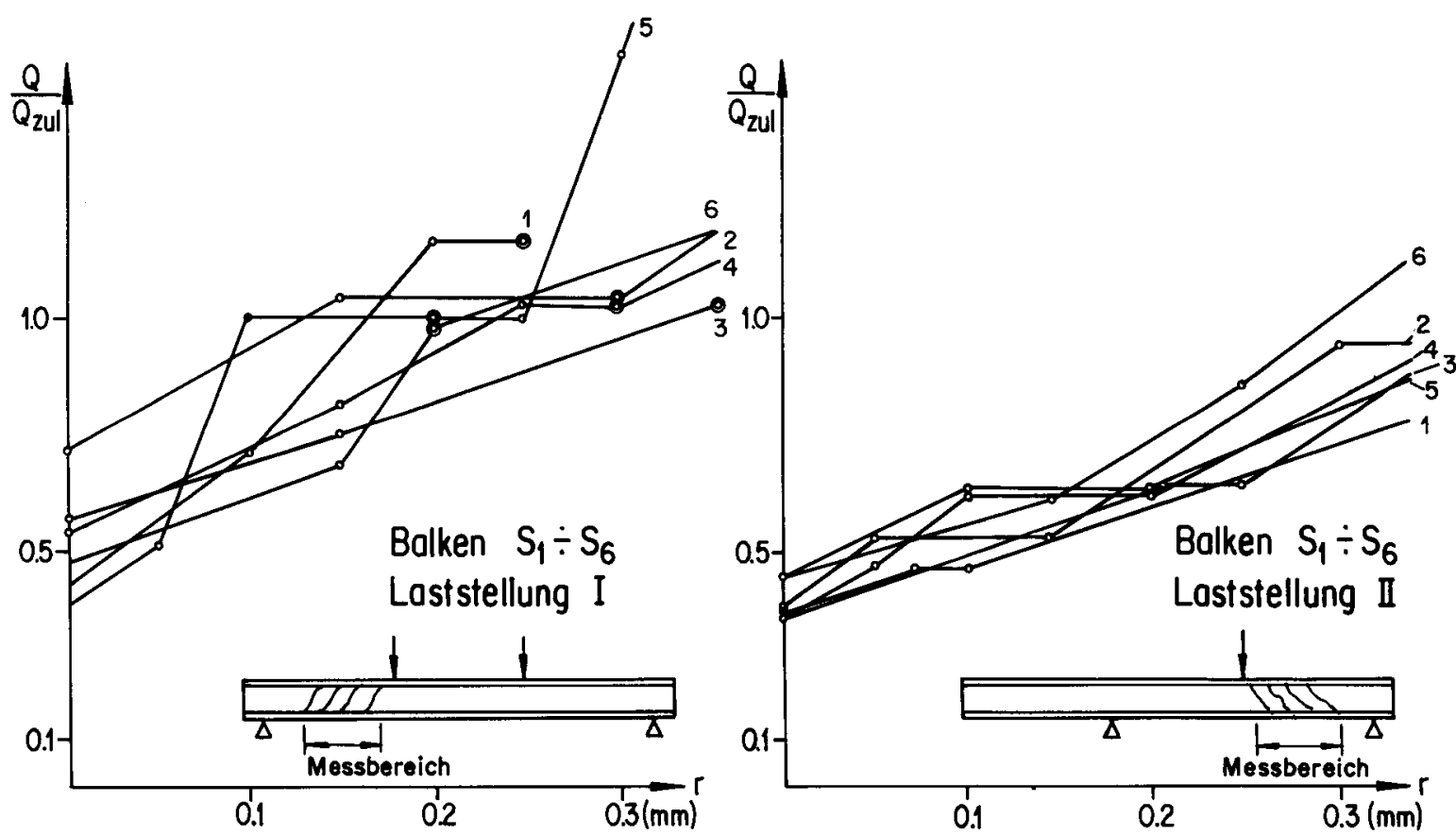

Bild 80: Maximale Rissbreiten bei kombinierter Belastung 


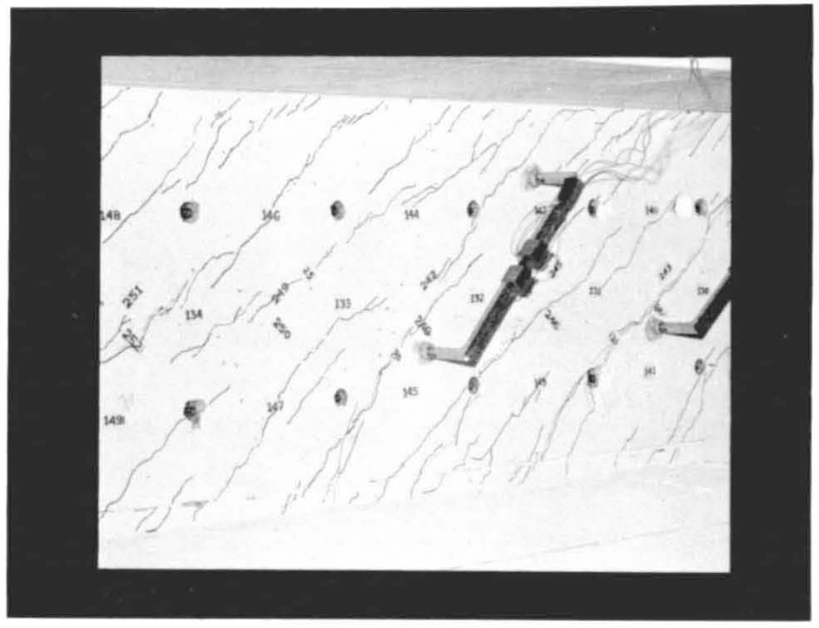

Druckseite LS 29

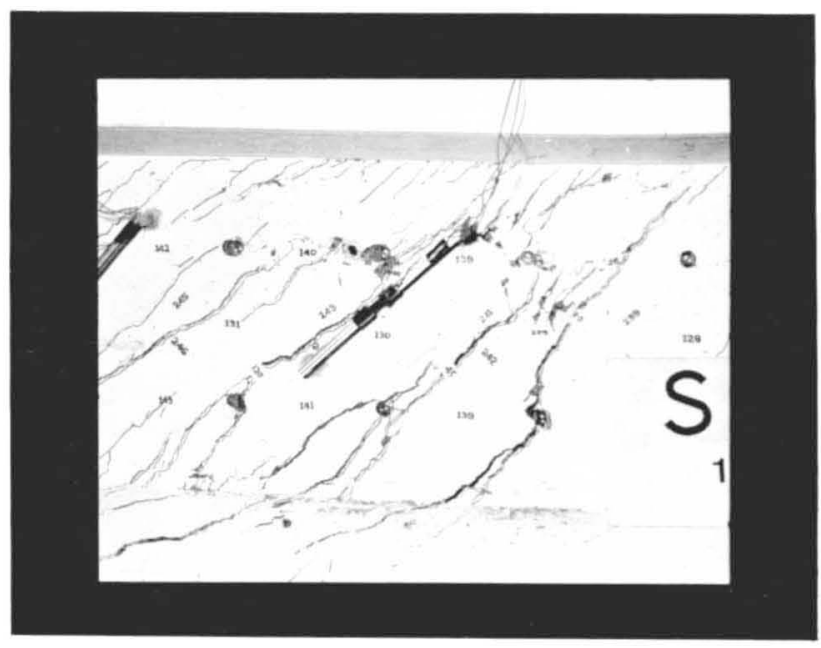

Druckseite LS 30

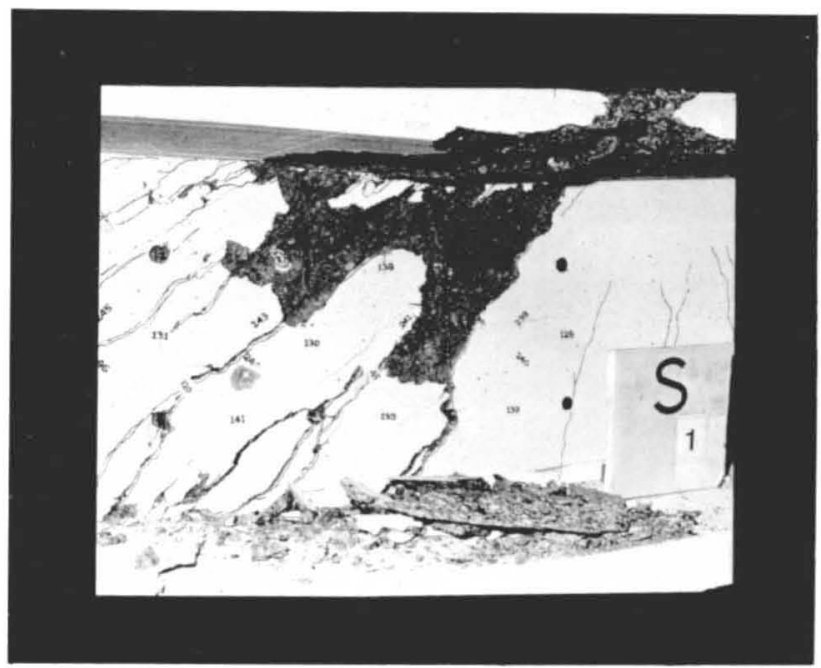

Druckseite nach Uberschreiten der Maximallasł

Bild 81: Entwicklung eines Sprödbruches: Balken $S_{1}$, Laststellung II 


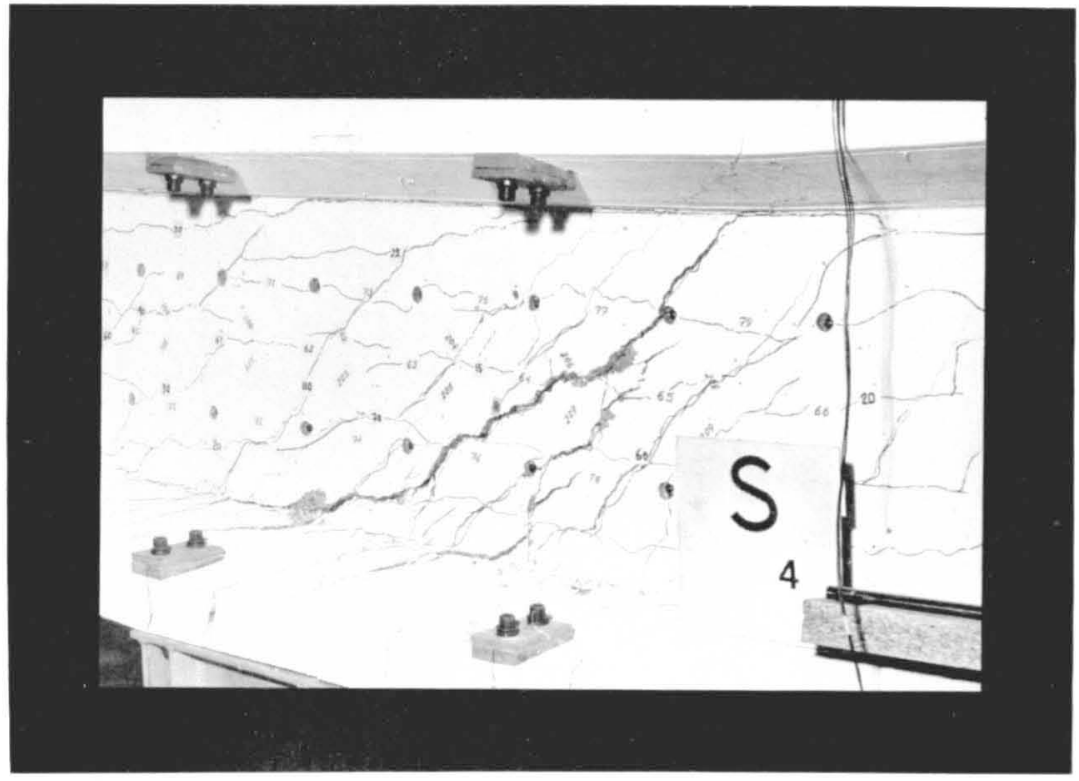

Bild 82: Duktiler Bruch Balken $\mathrm{S}_{4}$ Laststellung I

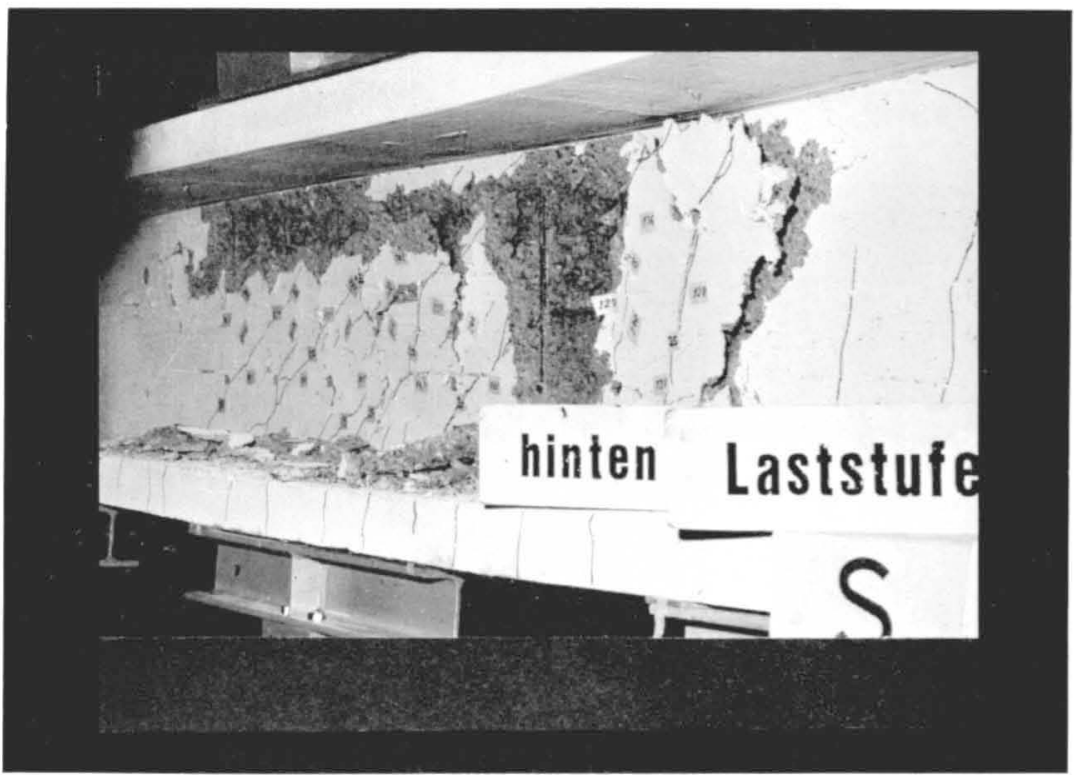

Bild 83: Spröder Bruch Balken $S_{6}$ Laststellung II 


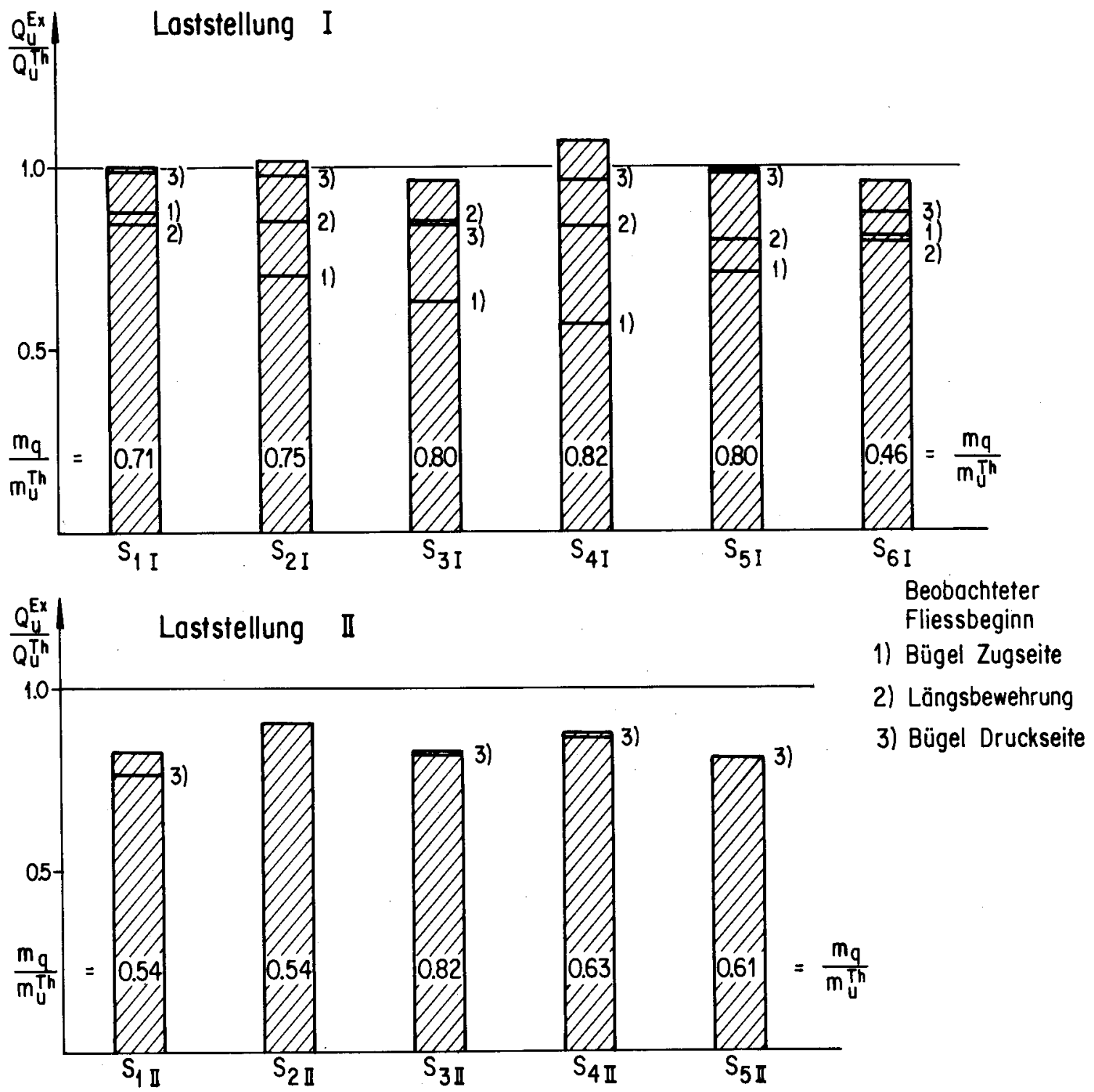

Bild 84: Theoretische und gemessene Bruchlasten

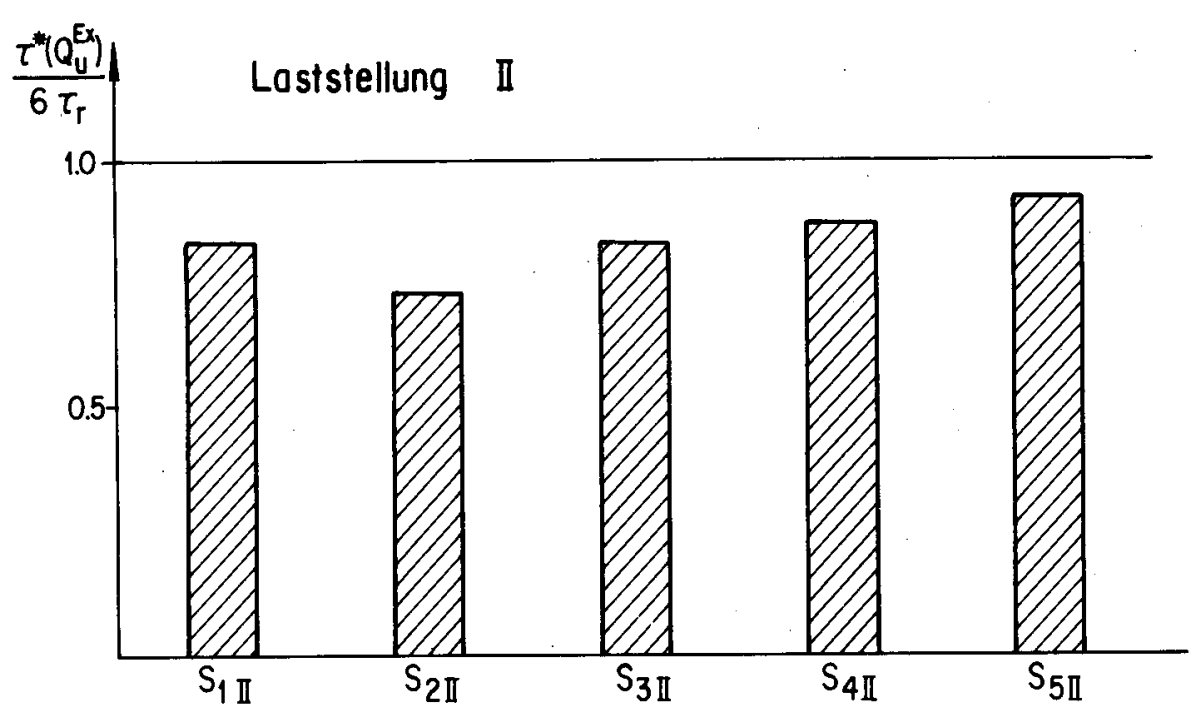

Bild 85: Vergleich der nominellen Schubspannung (bei Bruch) mit der oberen Schubspannungsgrenze 


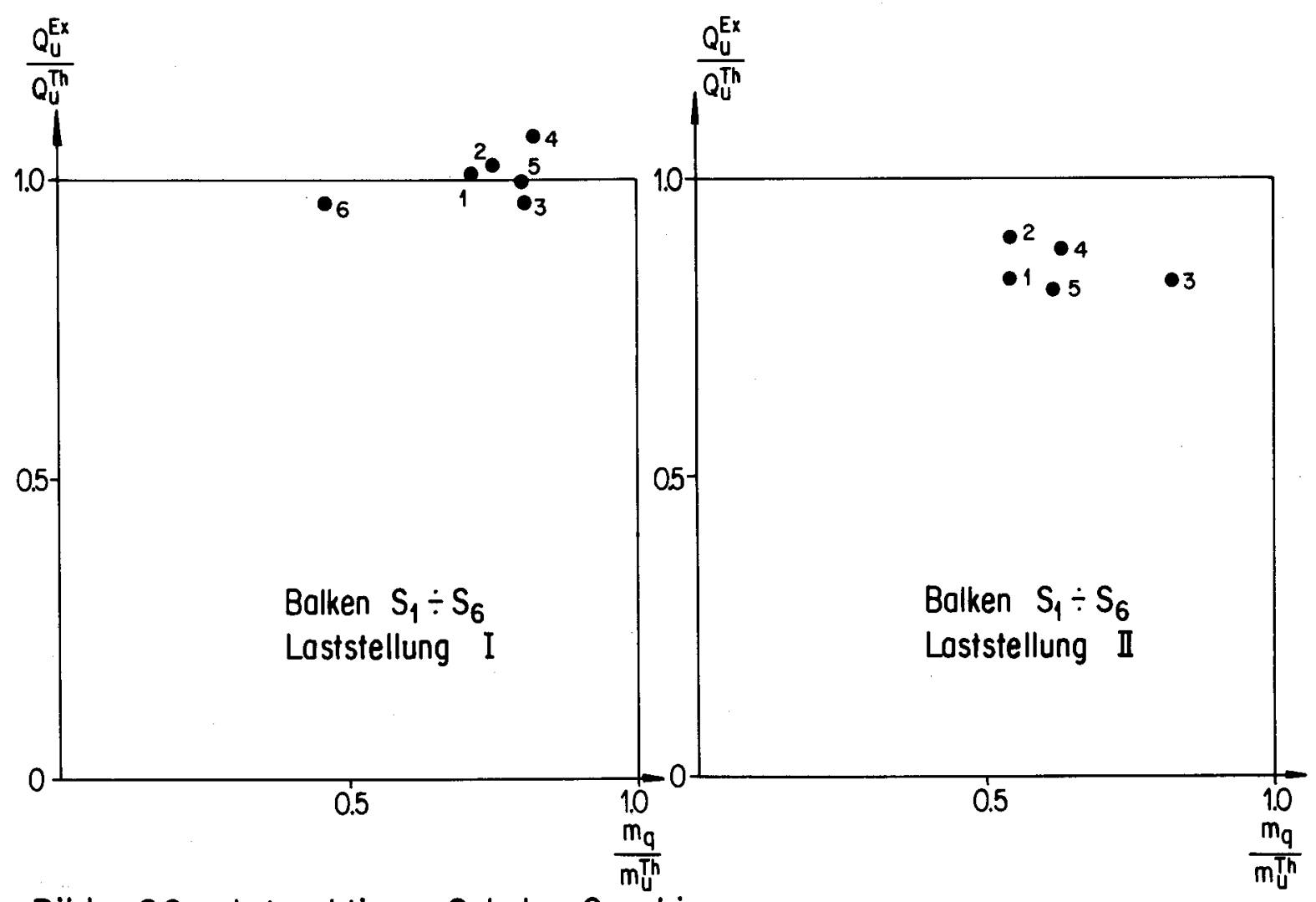

Bild 86: Interaktion Schub-Querbiegung

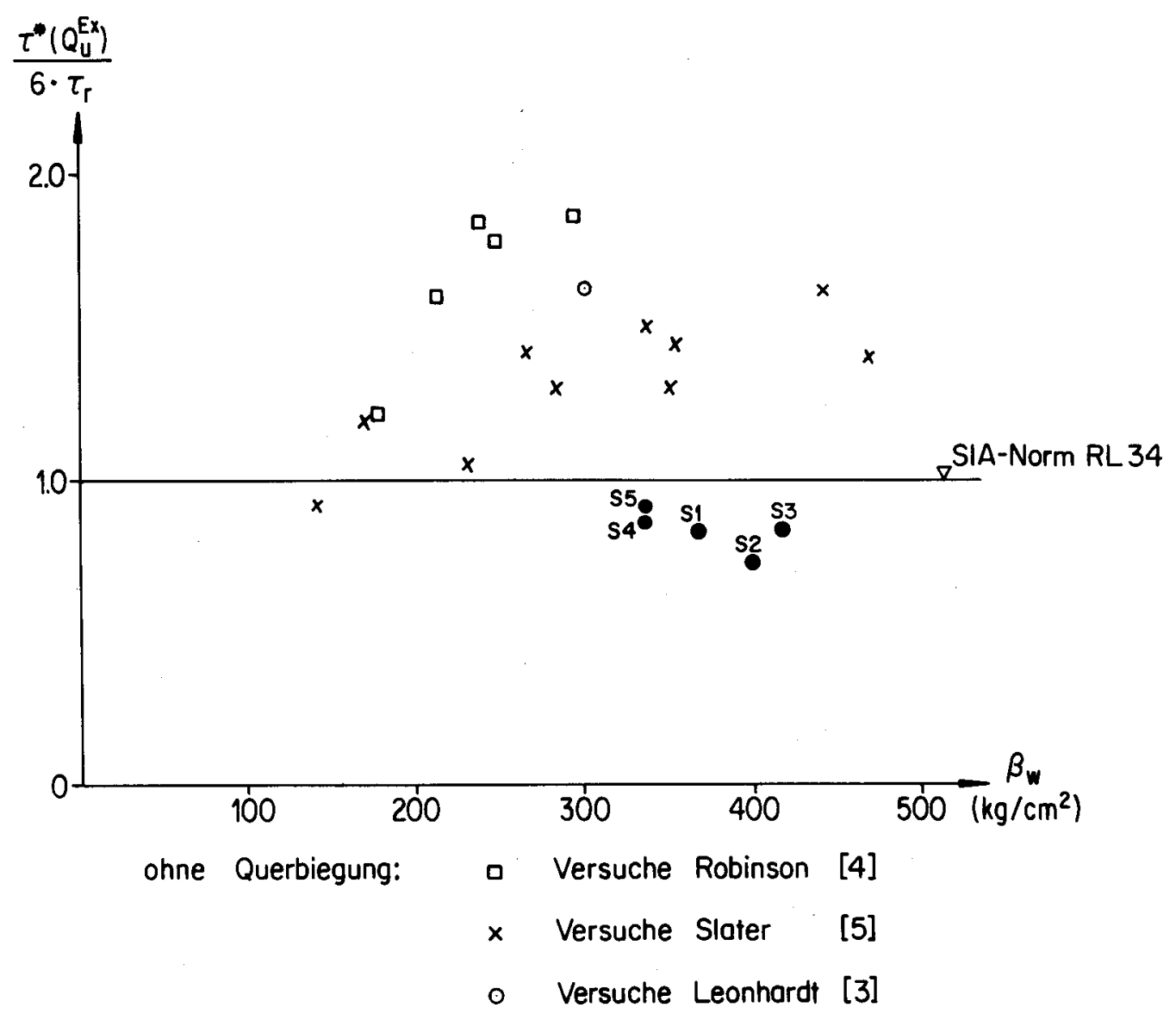

Bild 87: Sprödbrüche bei Schub mit und ohne Querbiegung 


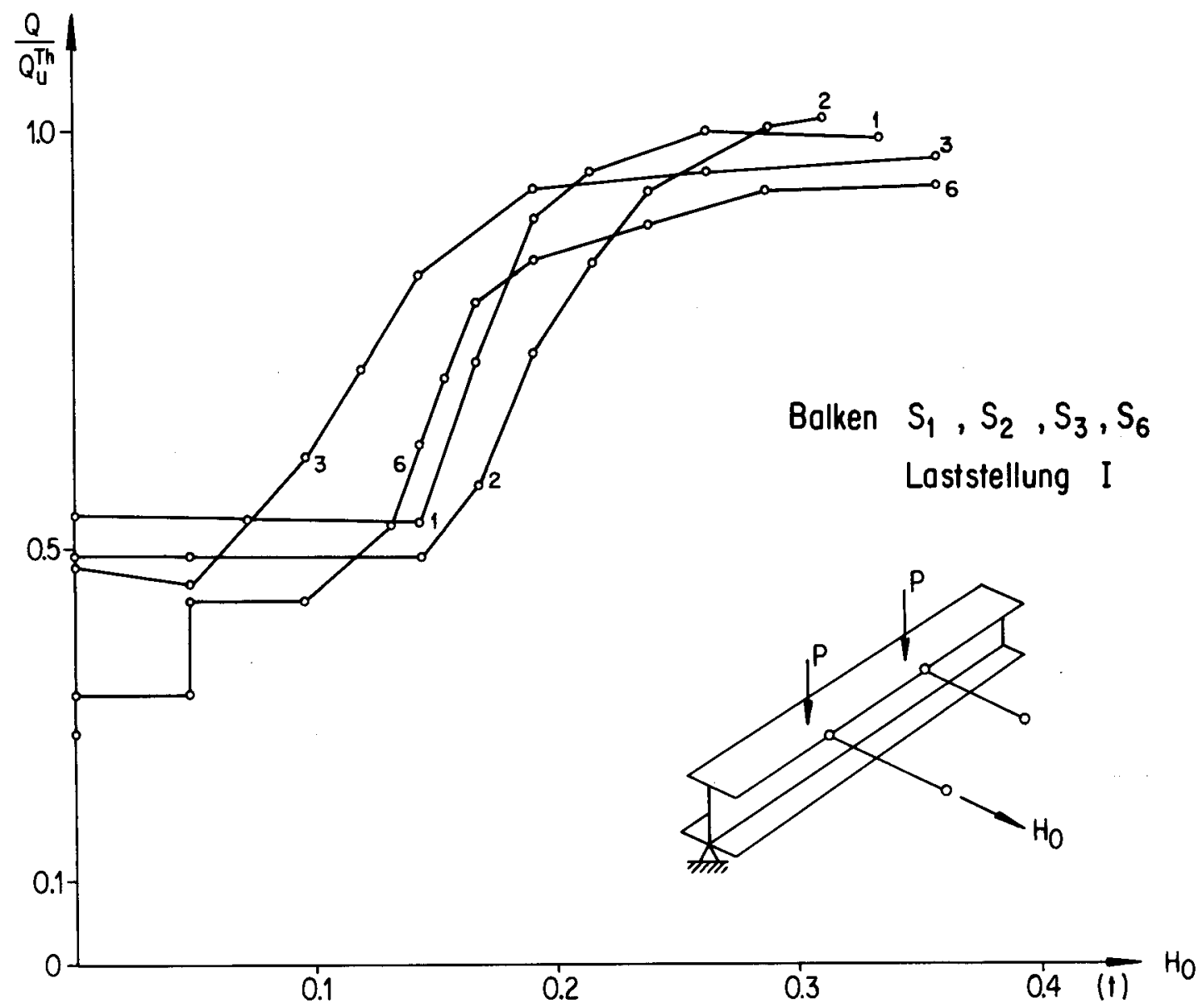

Bild 88: Seitliche Festhaltekraft $\mathrm{H}_{0}$ in Funktion der Querkraft

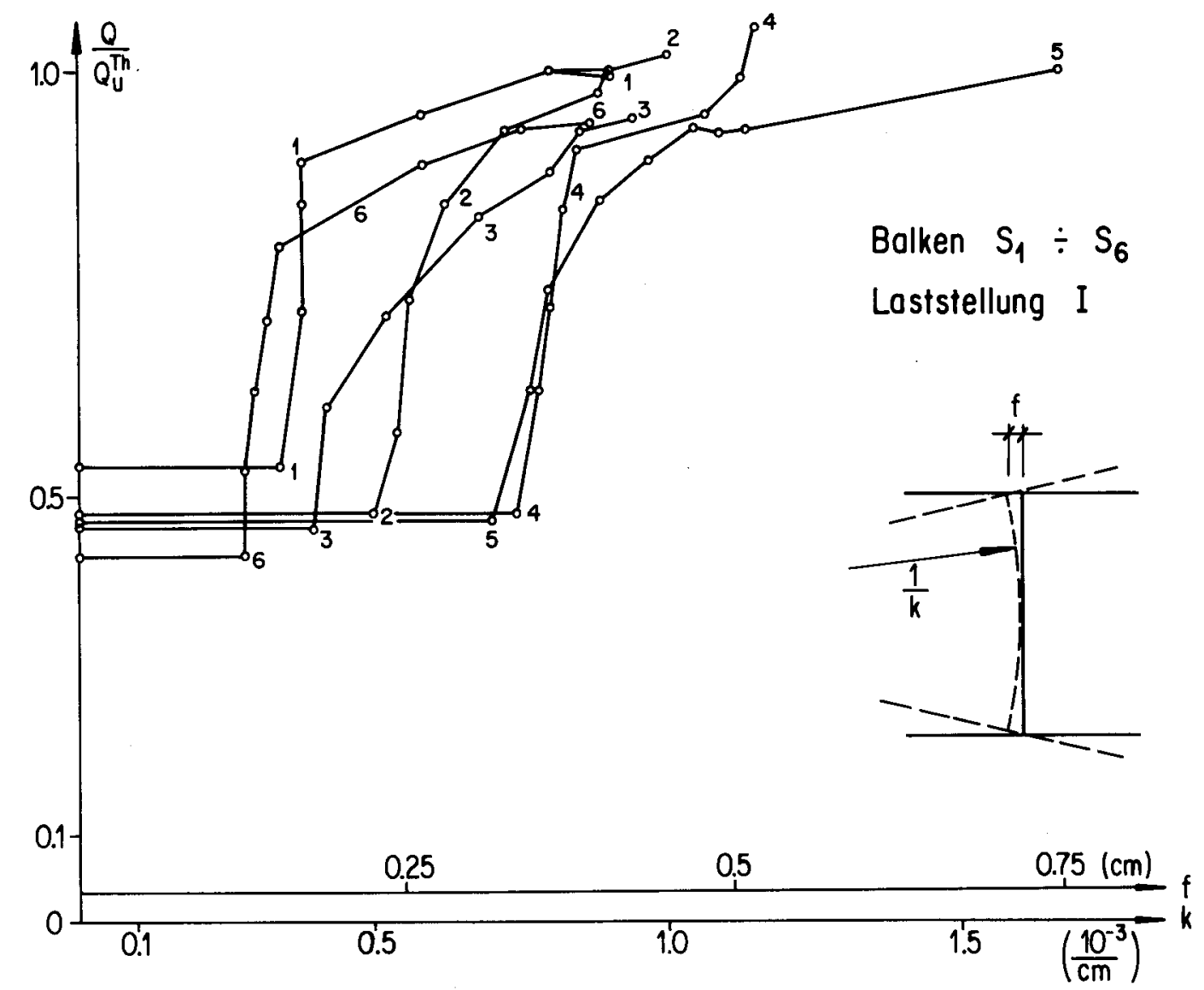

Bild 89: Krümmung des Steges in Funktion der Querkraft 


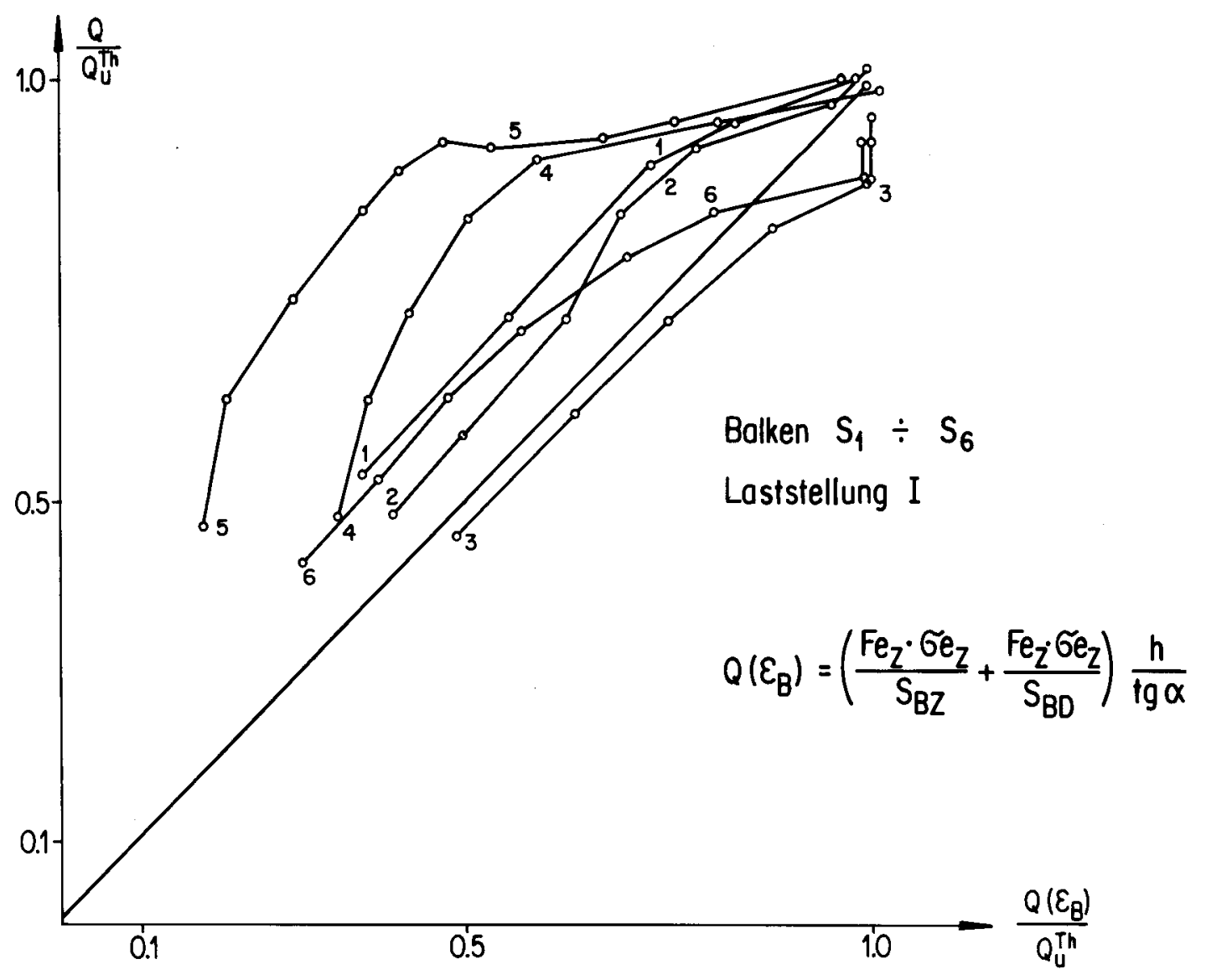

Bild 90: Vorhandene Bügelkräfte in Funktion der Querkraft
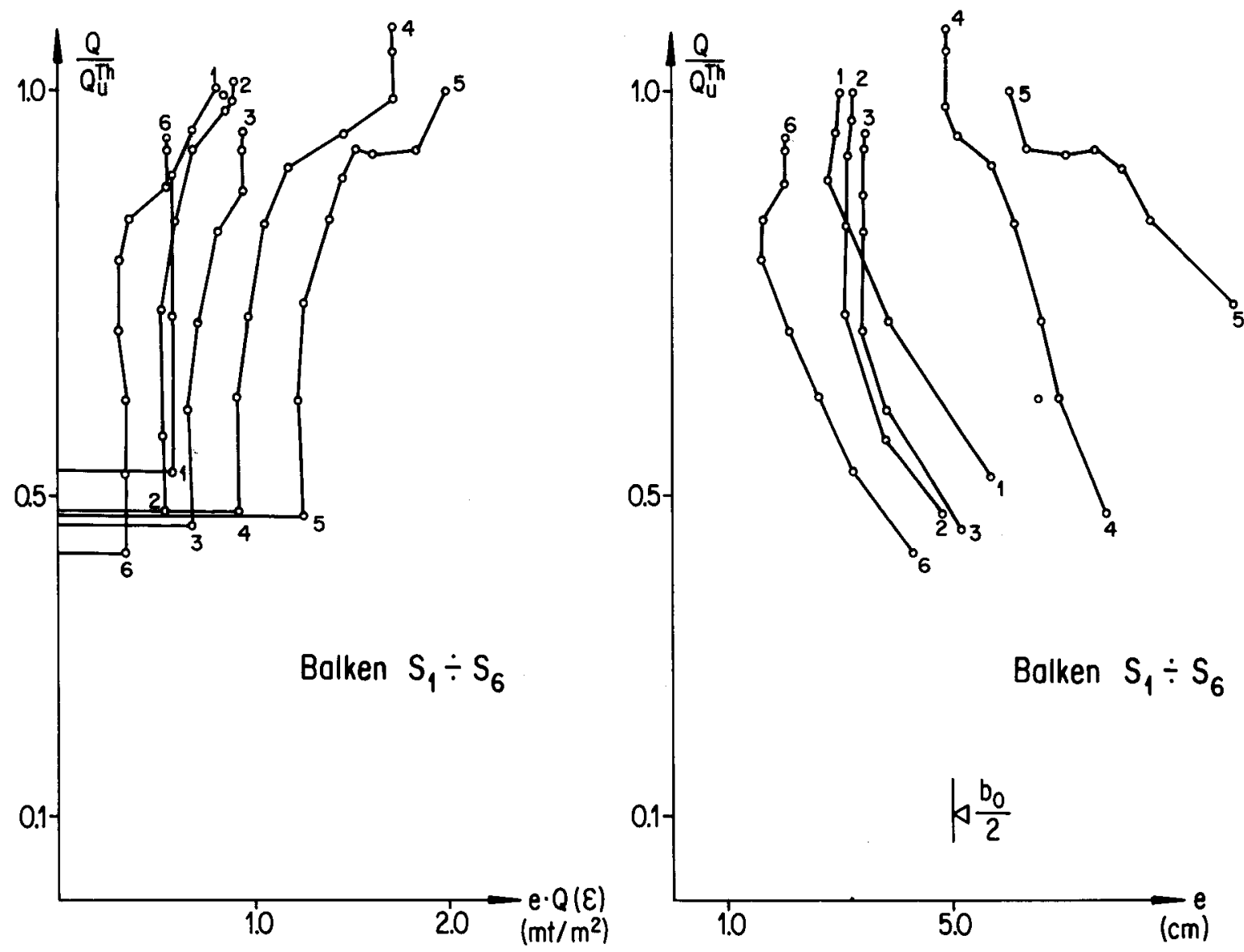

Bild 91: Exzentrische Querkraft 